\title{
Time-Resolved NMR-Spectroscopic Studies of
}

\section{Conformational Dynamics in DNA G-Quadruplexes}

\author{
Dissertation \\ zur Erlangung des Doktorgrades \\ der Naturwissenschaften \\ vorgelegt beim Fachbereich Biochemie, Chemie und Pharmazie \\ der Johann Wolfgang Goethe-Universität \\ in Frankfurt am Main \\ von \\ J. Tassilo Grün \\ geboren in Wetzlar
}

Frankfurt am Main

2021

(D30) 

Vom Fachbereich Biochemie, Chemie und Pharmazie der Johann Wolfgang Goethe-Universität als Dissertation angenommen.

Dekan: $\quad$ Prof. Dr. Clemens Glaubitz

Gutachter: $\quad$ Prof. Dr. Harald Schwalbe

Prof. Dr. Alexander Heckel

Datum der Disputation: $\quad$ 24.06.2021 



\section{Table of Contents}

List of Abbreviation iv

\section{Summary and Overview}

1.1 Summary 1

1.2 Zusammenfassung 3

\section{General Introduction}

\subsection{Structure and Dynamics of DNA G-Quadruplexes}

2.1.1 Molecular Structure and Conformations of DNA ............................................ 9

2.1.1 Non-Canonical DNA Structures....................................................................... 11

2.1.2 Canonical Structural Polymorphism............................................................... 14

2.1.3 Non-Canonical Structural Polymorphism...................................................... 15

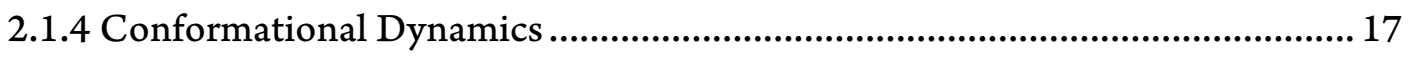

\subsection{Folding Dynamics of DNA G-Quadruplexes}

2.2.1 Folding Energy Landscapes and Folding Pathways ...................................... 19

2.2.2 Experimental Approaches to Measure Folding Kinetics .................................. 21

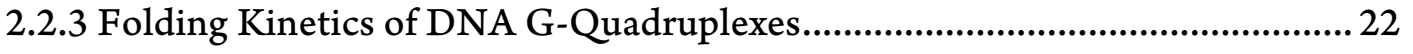

2.2.4 Comparison to RNA G-Quadruplexes.............................................................. 23

2.2.5 Mutual Exclusive Formation of DNA i-motifs and G-Quadruplexes............... 23

\subsection{Function and Targetability of DNA G-Quadruplexes}

2.3.1 Occurence of G-Quadruplex Forming Sequences in the Human Genome .... 24

2.3.2 Transcriptional Regulation of G-Quadruplexes and Binding-Interactions .... 26

2.3.3 Oxidative Damage under Cellular Stress Conditions ......................................30

2.3.4 Strategies in Anti-Cancer Treatment............................................................... 31

\subsection{The cMYC Proto-Oncogene Promoter}

2.4.1 The cellular Myelocytomatosis Protooncogene .................................................. 33

2.4.2 Nuclease Hypersensitive Element $\mathrm{III}_{1}$.............................................................. 35

2.4.3 Structures and Dynamics of the $c M Y C$ G-Quadruplex...................................... 37

2.4.4 Targeting the $c M Y C$ G-Quadruplex................................................................... 40

\subsection{NMR Methods to Study Structure and Dynamics in Nucleic Acids}

2.5.1 Structure Determination of Nucleic Acids with Nat. Abundance Isotopes.... 43

2.5.2 The NMR-Timescale: Methods to investigate Dynamics................................ 45

2.5.3 Real-Time NMR: Non-Equilibrium Dynamics ................................................. 47

2.5.4 Conformational Selection with Photolabile Protecting Groups ...................... 49 


\section{Materials and Methods}

3.1 DNA Oligonucleotides and Sample Preparation

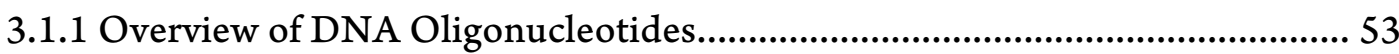

3.1.2 Synthesis of Photocaged DNA Oligonucleotides ........................................... 54

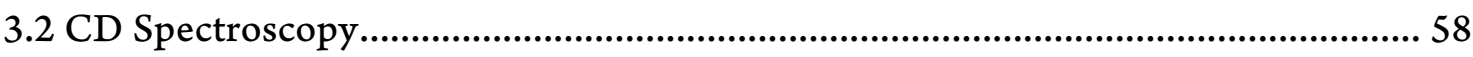

3.3 Non-Linear Regression and Global Fitting of Kinetic Data.................................... 58

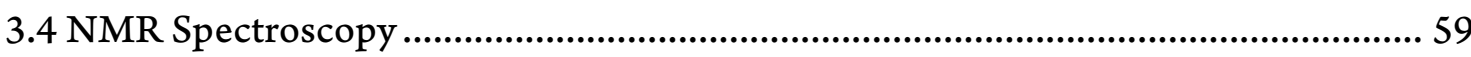

\section{G-Register Exchange Dynamics}

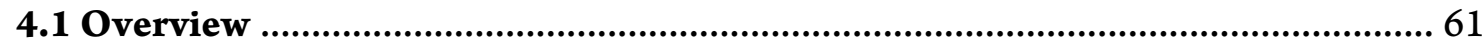

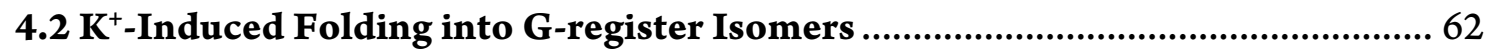

\subsection{Light-Induced Refolding of Caged G-Register Isomers}

4.3.1 Conformational Selection of G-Register Isomers ........................................... 64

4.3.2 Refolding into a Two-State G-Register Ensemble .......................................... 67

4.3.3 Refolding into a Full Set G-Register Ensemble.............................................. 68

4.4 G-Register Polymorphism in the hTERT Promoter G-Quadruplex................... 69

4.5 Activation Energies of G-Register Exchange Transitions ............................... 71

\subsection{Folding Initiation in Pre-Equilibrated $\mathrm{K}^{+}$-Solution}

4.6.1 Dynamics of Thermally-Induced (Un)-Folding of G-Register Isomers ........ 73

4.6.2 Light-Induced Folding into G-register Isomers .............................................. 75

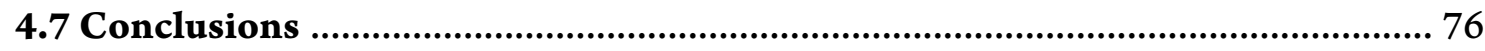

\section{Spare-Tire Exchange Dynamics}

5.1 Overview

\subsection{Light-Induced Folding of Caged Spare-Tire-Isomers}

5.2.1 Trapping Compl. Unfolded Spare-Tire Isomers with Multiple Photocages .. 80

5.2.2 Light-Induced Folding Kinetics under Pre-Equilibrated $\mathrm{K}^{+}$Conditions ....... 81

5.2.3 Intermediate Formation and Folding Pathway for $c M Y C-1234$..................... 84

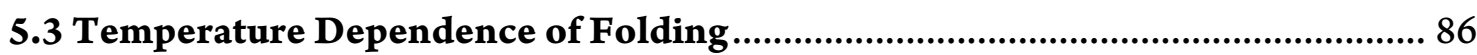

\subsection{Light-Induced Refolding of Caged Meta-Stable Minor Conformations}

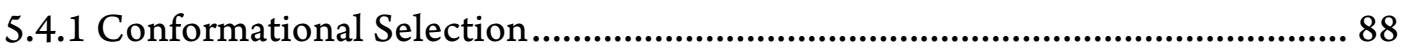

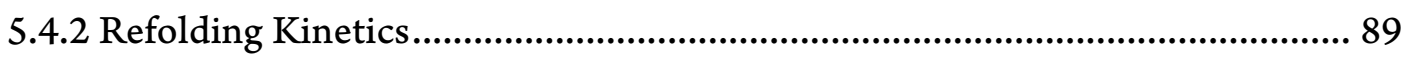


6 The Dynamic Behaviour of the cMYC Promoter G-Quadruplex

6.1 Folding Pathways for the 22 nt $c M Y C$ Full Length Sequence

6.1.1 K $\mathrm{K}^{+}$-Recruitment During Folding ...................................................................... 93

6.1.2 Parallel Reaction Pathways Accelerate Folding (G-Register Isomers) .......... 94

6.1.3 Enlarged Conformational Space Decelerates Folding (Spare-Tire Isomers) . 95

6.2 Folding intermediates .95

6.3 Refolding Across Different Transitory Ensembles.............................................. 97

6.4 Non-Canonical Structural Polymorphism as Challenge ...................................... 98

6.5 Model for cMYC G4 (Re)-Folding including Non-Canonical Polymorphism .. 99

6.6 Conclusion and Outlook 100

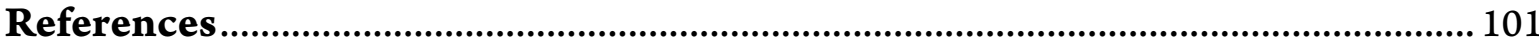

\section{Appendix}

6.1 HMBC and Deuterium Exchange NMR Spectrum 121

6.2 Plots of Time-Resolved 1D ${ }^{1} \mathrm{H}$ NMR Spectra for Spare-Tire G4 Folding ..................... 122

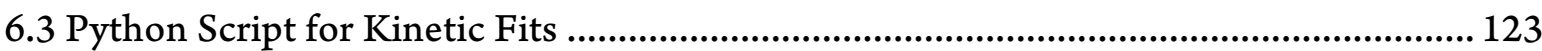

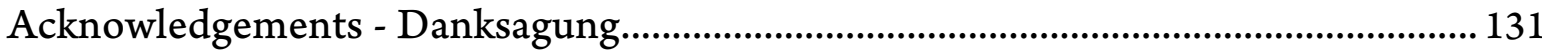

Curriculum Vitae 


\section{List of Abbreviations}

\begin{tabular}{|c|c|c|c|}
\hline $\mathbf{A}$ & Adenosine & MS & Mass spectrometry \\
\hline $\mathbf{b}(\mathbf{p})$ & Base (pair) & n.a. & not assigned \\
\hline C & Cytosine & n.d. & not determined \\
\hline CD & Circular Dichroism & NMR & Nuclear Magnetic Resonance \\
\hline COSY & Correlation Spectroscopy & NOESY & Nuclear Overhauser \\
\hline DMSO & Dimethyl sulfoxide & & Spectroscopy \\
\hline DNA & Deoxyribonucleic Acid & ns & number of scans \\
\hline \multirow[t]{2}{*}{ dNTP } & deoxy-Nucleoside-tri- & nt & Nucleotide \\
\hline & Phosphate & OD & optical density \\
\hline ds/ss & double/single stranded & PAGE & Poly Acrylamide Gel \\
\hline DSS & $\begin{array}{l}\text { 2,2-Dimethyl-2-silapentane-5- } \\
\text { sulfonic acid }\end{array}$ & PDB & $\begin{array}{l}\text { Electrophoresis } \\
\text { Protein Data Bank }\end{array}$ \\
\hline EDTA & $\begin{array}{l}\text { Ethylene Diamine Tetra } \\
\text { Acetic acid }\end{array}$ & $\begin{array}{l}\text { PNA } \\
\text { PPG }\end{array}$ & $\begin{array}{l}\text { peptide nucleic acid } \\
\text { photolabile protecting group }\end{array}$ \\
\hline \multirow{2}{*}{$\begin{array}{l}\text { ESI } \\
\text { et al. }\end{array}$} & Electrospray-Ionization & & Parts Per Million \\
\hline & et alia, and others & PQS & Putative Quadruplex \\
\hline \multirow[t]{2}{*}{ FRET } & Förster Resonance Energy & & Sequence \\
\hline & Transfer & RNA & Ribonucleic Acid \\
\hline $\mathbf{G}$ & Guanosine & rt & Room temperature (298 K) \\
\hline G4 & G-Quadruplex & $\mathbf{T}$ & Thymidine \\
\hline HSQC & $\begin{array}{l}\text { Heteronuclear Single } \\
\text { Quantum Coherence }\end{array}$ & TEA & $\begin{array}{l}\text { Tetraethylammonium } \\
{\left[\left(\mathrm{C}_{2} \mathrm{H}_{5}\right)_{4} \mathrm{~N}^{+}\right]}\end{array}$ \\
\hline НМВС & $\begin{array}{l}\text { Heteronuclear Multiple Bond } \\
\text { Correlation }\end{array}$ & TH & Thermal Hysteresis \\
\hline HPLC & $\begin{array}{l}\text { High Performance Liquid } \\
\text { Chromatography }\end{array}$ & TOCSY & $\begin{array}{l}\text { Total Correlation } \\
\text { Spectroscopy }\end{array}$ \\
\hline $\mathbf{K}$ & Kelvin & TRIS & $\begin{array}{l}\text { Tris-(hydroxymethyl)- } \\
\text { aminomethane }\end{array}$ \\
\hline $\mathbf{K}_{\mathbf{d}}$ & Dissociation constant & TSS & Transcription start site \\
\hline $\mathbf{K}-\mathbf{P}_{\mathbf{i}}$ & potassium phosphate buffer & $\mathbf{U}$ & Uridine \\
\hline MALDI & Matrix-assisted Laser & UV-vis & Ultraviolet-visible \\
\hline
\end{tabular}


"One of the defining characteristics of a living system is the ability of even the most intricate of its component molecular structures to self-assemble with precision and fidelity. Uncovering the mechanisms through which such processes take place is one of the grand challenges of modern science."

Sir Christopher M. Dobson, 2003 $3^{1,2}$ 



\subsection{Summary}

\section{Summary and Overview}

\subsection{Summary}

The present thesis Time-Resolved NMR-Spectroscopic Studies of Conformational Dynamics in DNA G-Quadruplexes deals with the detailed investigation of important structural dynamics in non-canonical secondary structure elements in nucleic acids.

G-rich DNA sequences in the human genome can form non-canonical secondary structures that deviate from the double stranded Watson-Crick helix. In presence of monovalent cations, G-residues are able to form tetrads via G-G Hoogsteen base pair interactions that stack to structures known as G-quadruplexes (G4, chapter 2.1). G4s are highly dynamic structures able to adopt numerous conformations. The pronounced polymorphism and the complex folding energy landscapes of DNA G4s lead to the co-existence of different folded conformations (chapter 2.2). The involved folding and refolding dynamics, described by their transition kinetics, remain largely enigmatic. The intrinsic dynamic and volatile nature of DNA G4s is a crucial feature for their vital roles in cell regulation and gene expression (chapter 2.3). Malfunction of the G4 biological functions is strongly linked to rare hereditary diseases, epigenetic regulation and oncogenesis. DNA G4s therefore have evolved as valuable targets in structure-based drug design. Understanding the conformational dynamics of G4s is crucial to enable and aid such therapeutic approaches. The human $c M Y C$ oncogene is a key proliferation driver in numerous cancer types. A nuclease hypersensitive element $\left(\mathrm{NHE}-\mathrm{III}_{1}\right)$ in the cMYC promoter region is able to fold into a polymorphic G4 ensemble that regulates up to $90 \%$ of cMYC transcription levels (chapter 2.4). The $c M Y C$ NHE-III ${ }_{1}$ G4 ensemble features two different kinds of newly discovered non-canonical polymorphism: (i) the sequence has more than four G-rich tracts ("spare-tires") close in sequence that can isomerize by involving different G-tracts into their most stable structure; and (ii) in addition, two of the individual G-tracts contain more than three consecutive G-residues. Different G-residues within one G-tract can participate in the tetrad formation opening the possibility of G-register isomerism. The kinetics and dynamics of G4 formation (folding) and transformation (refolding) featuring both kinds of non-canonical polymorphism have remained completely elusive. This thesis presents a novel approach to investigate this kinetics with atomic resolution using time-resolved NMR methods (chapter 2.5). A general method to prepare and trap unfolded (conformational suppression) or isolated folded (conformational selection) states utilizing photolabile protecting groups is presented.

Chapter 4 examines the dynamics of G-register isomers. The kinetics of folding (starting from unfolded states) and refolding (starting from isolated folded states) have been investigated. The refolding kinetics for two different G4 pairs are presented, each pair is related via G-register isomerism: (i) two co-existing all-parallel G4s found in the $c M Y C$ promoter; and (ii) two co-existing hybrid and parallel G4s found in the $h T E R T$ promoter. 


\subsection{Summary}

$\mathrm{K}^{+}$-induced folding into a two-state G-register pair reveals a kinetic partitioning mechanism. Both isomers are folded concurrently, with a kinetic overshoot of one isomer. Subsequent to initial folding, slow refolding kinetics $\left(\sim 0.9 \mathrm{~h}^{-1}\right)$ have been observed for the relaxation towards conformational equilibrium. A careful analysis of the involved kinetics and apparent activation energy barriers supports the hypothesis of inherently different refolding mechanisms for the hTERT and cMYC G4s. The timeresolved NMR data are accompanied by experimental data from a biophysical method that is based on analysis of thermal hysteresis for thermal (un)-folding of the respective G4 oligonucleotides.

Chapter 5 explores the dynamics of spare-tire isomers. The kinetics of all relevant folding and refolding trajectories for three cMYC spare-tire G4s $(1234,1245$, and 2345) each involving four of the five possible strands have been unravelled. The folding kinetics are vastly different, with the fastest conformation (2345) folding four times $\left(\sim 400 \mathrm{~h}^{-1}\right)$ faster than the slowest conformation (1234). Interestingly, the temperature dependence is markedly different for each of the three isomers. For 1234, a positive apparent activation energy barrier is observed. Folding of this conformation involves the formation of off-pathway intermediates as kinetic traps. The intermediate state was spectroscopically scrutinized and a reasonable structural model could be proposed, based on experimental findings. 2345 shows optimized fast kinetics proceeding close to funnel-like, without observable kinetic trapping. The negative folding activation barrier indicates a mainly entropically driven folding process. 1245 features a long internal loop. The folding kinetics of 1245 show an unprecedented non-Arrhenius temperature dependence. It was shown that the 1245 conformation is a rare interjacent case for the two limiting regimes on the folding energy landscape observed for 1234 (pronounced kinetic partitioning) and 2345 (funnel-like folding). Refolding kinetics between the different spare-tire isomers complete the analysis of the individual pathways along the conformational energy landscape of the entire $c M Y C$ G4 ensemble. Refolding is slow $\left(0.16-0.30 \mathrm{~h}^{-1}\right)$ and results into a complete refolding into the 2345 conformation, which allows denoting 1234 and 1245 as long-lived meta-stable conformations.

Chapter 6 presents a comprehensive discussion of all experimental data within this thesis. The discussion further evaluates the findings of this thesis in the context of the recent literature. The bias in the comparison of crucial experimental conditions (e.g. $\mathrm{K}^{+}$-recruitment or possible pre-folded states) highlight the great advantages of the presented methodological approach over previously reported studies on G4 structural dynamics. Finally, a kinetic model is proposed that allows the disentanglement of key kinetic steps in for all relevant folding and refolding trajectories within the cMYC G4 ensemble. This model explains the different observed kinetics based on structural considerations for intermediate states and possible transitory ensembles. 


\subsection{Zusammenfassung}

\subsection{Zusammenfassung}

Die vorliegende Arbeit Zeitaufgelöste NMR-spektroskopische Untersuchung konformationeller Dynamiken in DNA G-Quadruplexen befasst sich mit der detaillierten biophysikalischen Untersuchung wichtiger strukturdynamischer Eigenschaften von nicht-kanonischen Nukleinsäure Sekundärstrukturelementen.

Im Genom aller eukaryotischer Lebewesen, insbesondere dem menschlichen Genom finden sich DNA-Sequenzabschnitte, die überdurchschnittlich Guanosin (G)-reich sind. Diese poly-G Abschnitte sind nicht zufällig im Genom verteilt, sondern häufen sich vermehrt in Genabschnitten, die besonders wichtig für die Regulation der Genexpression sind. G-reiche DNA-Sequenzen können unter geeigneten Umständen alternative Sekundärstrukturen ausbilden, die von der doppelsträngigen, kanonischen Watson-Crick Konformation abweichen. In Anwesenheit monovalenter Kationen können sich G-Nukleotide in einer Tetrade über Hoogsteen Interaktionen anlagern. Diese Tetraden können sich stapeln und dadurch sogenannte G-Quadruplexe (G4) ausbilden.

G-Quadruplexe haben durch ihre ungewöhnliche Struktur Einfluss auf die Transkription und Replikation, sowie DNA-Protein Interaktionen und die Genomstabilität insgesamt. G4 Strukturelemente sind dabei sehr dynamisch im zellulären Umfeld, sie werden ständig gefaltet, umgefaltet und entfaltet. Eine Vielzahl molekularer Mechanismen steuert und reguliert die Ausbildung von G4 und deren Einfluss auf die gesunde Zell-Homöostase. Eine Fehlregulierung dieser G4 Mechanismen ist assoziiert mit unter anderem der Pathogenese einiger Erbkrankheiten, epigenetischen Einflüssen, sowie der Karzinogenese verschiedener Tumorerkrankungen. Die Untersuchung der Dynamiken von Faltung und Umfaltung von G4, die an der Genregulation beteiligt sind, ist daher von fundamentaler Bedeutung.

Das menschliche $c M Y C$ Gen wird typischerweise als proto-Onkogen bezeichnet. Es kodiert für einen unspezifischen Transkriptionsfaktor, der bei einer Vielzahl von systematischen und soliden Tumorerkrankungen stark überexprimiert wird. Die zelluläre Konzentration des Genprodukts kann zu 90\% über ein G4 cis-Element in der Promotorregion reguliert werden; das Protein selbst ist jedoch nicht mit herkömmlichen niedermolekularen Wirkstoffen angreifbar. Aufgrund dieser Erkenntnisse hat sich das G4 cis-regulierende Element im cMYC Promotor als vielversprechendes Wirkstoffziel für strukturbasiertes Wirkstoffdesign etabliert.

Der cMYC G4 hat die Möglichkeit verschiedene Konformationen einzunehmen, was auch für viele andere G4 gilt. Dieser Polymorphismus zeichnet sich typischerweise durch verschiedene Faltungstopologien aus. Im Falle des cMYC G4 kann man zusätzliche, nicht-konventionelle Formen der konformationellen Isomerie finden. Zum einen gibt es die Möglichkeit, dass bei einem G4, der aus drei Tetraden und vier intramolekularen Strangabschnitten (dreistöckiger G4) besteht, einzelne Strangabschnitte mehr als drei konsekutive G-Nukleotide besitzen. Dadurch können sich Faltungs- 
Isomere bilden, die sich durch Verschieben des Strangs relativ zum verbleibenden dreistöckigen Tetradengerüst ergeben. Man spricht von G-Register Isomeren. Eine zweite Möglichkeit der Strukturisomerie ergibt sich, wenn in einer Nukleotidsequenz mehr als vier G-reiche Strangabschnitte aufeinander folgen. Jeweils vier dieser Strangabschnitte können in unterschiedlicher Weise kombiniert werden, um ein G4 Isomer auszubilden. In jedem dieser so zustande gekommenen G4 verbleibt ein (oder mehrere) G-reicher Strangabschnitt, der im konkreten Isomer nicht zur Faltung verwendet wird. Diese zusätzlichen G-Stränge werden daher auch Ersatzräder (engl. spare-tires) genannt; man erhält spare-tire Isomere.

Obwohl diese Formen des Polymorphismus, deren biologischer Kontext und die biophysikalischen Konsequenzen in Arbeiten von C. Burrows (2015) und A. Mittermaier (2016) erstmals umfassend beschrieben wurden, gab es bis zum Ausgangspunkt dieser Arbeit keine Kenntnisse über deren strukturelle Dynamik, den Faltungswegen und den zugrundeliegenden molekularen Mechanismen. Die Erkenntnisse, die über die konformationellen Dynamiken des cMYC G4 Elements gewonnen wurden und hier vorgestellt werden, sind zum einen grundlegender Natur. Die Faltung von G4 ist ebenso wie beispielsweise die Faltung von Proteinen ein komplexer und faszinierender Vorgang, der weder in der theoretischen noch in der experimentellen Beschreibung bislang hinreichend verstanden ist. Darüber hinaus erweitern die beschriebenen konkurrierenden Faltungskinetiken direkt das Verständnis der biologischen Funktionsweise und Aufrechterhaltung der Funktionsfähigkeit des cMYC G4 Elements in zellulärer Umgebung. In der Literatur gibt es zahlreiche Hinweise darauf, dass die $c M Y C$ G4 Sequenz unter zellulären Stressbedingungen leicht durch Oxidation der G-Nukleobasen geschädigt oder durch Mutationen verändert werden kann. Zudem wird das cMYC G4 Element von zahlreichen Proteinen erkannt und gebunden, wobei die Bindungsaffinitäten Konformationsspezifisch sehr stark variieren. Der ausgeprägte Polymorphismus ist also Teil der Regulation und Funktionalität des $c M Y C$ G4 Elements, wodurch die Adaption an eine veränderte zelluläre Umgebung gewährleistet wird.

Zeitaufgelöste Kernspinresonanz (engl. nuclear magnetic resonance, NMR) Spektroskopie ist eine bestens geeignete Methode, um die Dynamik von Biomakromolekülen mit atomarer Auflösung zu studieren. Die Proben können dabei unter physiologischen Bedingungen und auf einer breiten Zeitskala untersucht werden. Typischerweise werden Echtzeit-Messungen solcher Dynamiken als Relaxationsprozess eines Nicht-Gleichgewichtszustands zurück in einen Gleichgewichtszustand untersucht. Um solche Experimente durchführen $\mathrm{zu}$ können, braucht es geeignete Herangehensweisen für die Präparation eines Nicht-Gleichgewichtszustands. In dieser Arbeit wird eine neu erarbeitete Strategie vorgestellt, die es erlaubt, Einblick in die Faltungs- und Umfaltungskinetiken eines dynamischen Konformations-Ensembles nicht-konventioneller Strukturisomere der $c M Y C$ G4 DNA-Sequenz zu erhalten. 


\subsection{Zusammenfassung}

Der Volllängensequenzabschnitt (hier: 22-mer) des $c M Y C$ G4 Elements umfasst fünf G-reiche Strangabschnitte, für diesen Abschnitt wurden drei Konformationen mit paralleler Faltungstopologie berichtet (D. Yang 2005, 2011, 2019). Im thermodynamischen Gleichgewicht unter physiologischen Bedingungen bildet sich fast ausschließlich diejenige Konformation, die sich aus den vier $3^{\text {‘-terminalen }}$ G-Strangabschnitten bildet. Zunächst wurde die Faltung dieser prädominanten $c M Y C$ Konformation untersucht, wobei eine 18-mer Oligonukleotidsequenz ohne den 5'-terminalen G-Strangabschnitt verwendet wurde. Diese Konformation liegt in einem Gleichgewicht zweier co-existenter SubKonformationen vor, die sich formal durch einfaches Verschieben eines Strangabschnitts ergeben (G-Register Isomere). Hierfür wurde ein ungefalteter Zustand erzeugt, indem $\mathrm{K}^{+}$-freie Bedingungen präpariert wurden. Durch sehr schnelle Zugabe von $\mathrm{K}^{+}$-Ionen kann die Faltung in situ induziert werden. Es zeigte sich, dass die beiden Sub-Konformationen parallel gebildet werden, wobei eines der G-Register Isomere zunächst kinetisch begünstigt wird und sich danach durch langsame Umfaltung das Gleichgewicht der Isomere einstellt.

Um tiefergehenden Einblick in die Umfaltung zu erhalten, wurde die Umfaltungskinetik direkt untersucht. Hierzu wurden photolabile Schutzgruppen (engl. Photocages) positionsspezifisch an bestimmten G-Nukleobasen ( $\left.\mathrm{O}^{6}-(\boldsymbol{R})-\mathrm{NPE}\right)$ angebracht. Die Schutzgruppen blockieren die Basenpaar-Interaktionen des Nukleotids, wodurch dieses sich nicht mehr an einer Tetradenbildung beteiligen kann. Die Photocages wurden jeweils an den Nukleotiden eingeführt, die nur in jeweils einem der G-Register Isomere an der Tetradenbildung beteiligt sind. Durch diese gezielte Destabilisierung konnten die Isomere getrennt und im gefalteten Zustand isoliert werden. Die so erhaltenen Konformationen wurden umfassend spektroskopisch charakterisiert. Es ergaben sich keine Änderungen im Vergleich zu den literaturbekannten NMR-Strukturen. Nach in situ LaserLichtanregung werden die Photocages abgespalten, wodurch das native, nicht modifizierte Oligonukleotid zurückerhalten wird. Es konnte beobachtet werden, dass sich das natürliche Gleichgewicht der Isomere zurückbildet und die Kinetik des Vorgangs konnte dekonvolutiert werden.

Dieser Ansatz wurde ausgeweitet auf eine weitere G4 DNA-Sequenz, die in zwei unterschiedlichen G-Register Isomeren vorliegen kann ( $h T E R T)$. Da sich hier (hybrid vs. parallel), anders als bei cMYC (parallel vs. parallel), die beiden Konformationen durch größere strukturelle Unterschiede auszeichnen, sollten Rückschlüsse auf vermutete unterschiedliche Umfaltungsmechanismen gezogen werden. Die Ratenkonstanten der Umfaltung beider G4 Systeme weichen jedoch nicht signifikant voneinander $\mathrm{ab}$, was zunächst überraschend scheint. Da sich die Aktivierungsenergie aus dem geschwindigkeitsbestimmenden Schritt der gesamten Umfaltung ergibt, konnte nicht direkt von der apparenten Umfaltungskinetik auf nachgelagerte, schnellere kinetische Schritte geschlossen werden.

Ein Vergleich der kinetischen Daten der Echtzeit-NMR Experimente mit Experimenten, die auf Hystereseeffekten bei thermischer (Ent)-Faltung beruhen, ergab jedoch wesentliche energetische 


\subsection{Zusammenfassung}

Unterschiede für die jeweiligen Umfaltungsprozesse. Dadurch konnten unterschiedliche Übergangszustände skizziert werden, die sich durch einen unterschiedlichen Grad der Entfaltung auszeichnen. Während der hTERT G4 fast vollständig entfaltet und anschließend in eine zweite Konformation rückfaltet, gleicht die Umfaltung des $c M Y C$ G4 vielmehr einem Verrücken des einzelnen Stranges, wobei die Gesamtstruktur weitestgehend erhalten bleibt. Die apparente Aktivierungsenergie beschreibt dabei das initiale Brechen der Wasserstoffbrückenbindungen für einen der G-Strangabschnitte.

Der Ansatz, das konformationelle Gleichgewicht durch Photocages transient zu stören, wurde daraufhin weiterentwickelt. Mehrere Photocages wurden an Nukleobasen in zentraler Position einzelner G-Strangabschnitte angebracht. Dadurch konnte eine ausreichende Destabilisierung erreicht werden, die die Faltung jedweder G4 Strukturen unterbindet. Somit wurde ein ungefalteter Zustand erzeugt, der unter ansonsten frei wählbaren, physiologischen Bedingungen besteht. Durch in situ Photolyse der Schutzgruppen konnte so die Licht-induzierte G4 Faltung unter konstanten Puffer- und Temperaturbedingungen untersucht werden. Dieser Ansatz wurde auf die Untersuchung der Faltungswege, die zu verschiedenen spare-tire Isomeren führen, fokussiert. Hierfür wurde die 22-mer Volllängensequenz des $c M Y C$ G4 Elements mit allen fünf G-reichen Strangabschnitten (von 5'-3', abgekürzt: 1-2-3-4-5) verwendet. Neben der prädominanten $c M Y C$ Konformation (mit den G-Strangabschnitten: 2-3-4-5, cMYC-2345) können so zusätzlich zwei weitere Konformationen gebildet werden, die im Gleichgewichtszustand unter physiologischen Bedingungen nur geringfügig populiert sind (cMYC-1234 und $c M Y C-1245)$.

Es zeigen sich signifikant langsamere Faltungskinetiken für die beiden Alternativ-Konformationen im Vergleich zur prädominanten Konformation. Eine temperaturabhängige Untersuchung dieser Kinetiken und anschließende Arrhenius-Analyse zeigte, dass den drei möglichen Konformationen drei fundamental unterschiedliche Faltungsmechanismen zugrunde liegen. Die prädominante Konformation $c M Y C-2345$ zeigt eine negative Aktivierungsenergie für die spontane Faltung. Dieser Prozess scheint daher entropisch getrieben zu sein. Die Möglichkeit paralleler Faltungswege, über G-Register Sub-Konformationen erhöht die konformationelle Entropie dieses KonformationsEnsembles zusätzlich. Dadurch wird die Faltungskinetik deutlich beschleunigt; die Energielandschaft gleicht einem Faltungstrichter. (Trichter-artige Faltung). Die Bezeichnung „Trichter-artig“ zur Beschreibung des Faltungsweges der prädominanten Konformation darf dabei nicht missverstanden werden. Denn auch hier konnten parallele, konkurrierende Faltungswege kinetisch differenziert werden, die zu weiteren Sub-Konformationen (G-Register Isomeren) führen. Der Kipppunkt für die distinkten Faltungswege der Sub-Konformationen liegt jedoch an einem weit fortgeschrittenen Zeitpunkt der Faltung. cMYC-1234 hingegen verzweigt sich bereits zu einem frühen Zeitpunkt in separate Faltungswege und bildet ein langlebiges Intermediat, das sowohl NMR-, als auch CDspektroskopisch nachgewiesen werden konnte. Die CD-Signatur des Intermediats zeigt, dass die 
Konformation in einer relativ zueinander anti-parallelen Anordnung (5'-3'-Richtung) der G-Strangabschnitte faltet. Da die stabile Konformation aller untersuchten cMYC G4 spare-tire Isomere jedoch in jedem Fall eine parallele Anordnung aller beteiligten G-Strangabschnitte relativ zueinander aufweist, muss dieses Intermediat folglich wieder aufgebrochen werden. Die apparente Aktivierungsenergie ist daher positiv und repräsentiert die Umfaltungsbarriere, die aus dieser kinetischen Falle überwunden werden muss. Der Faltungsmechanismus kennzeichnet sich durch ein komplex verzweigtes Netzwerk verschiedener Makrozustände entlang der Faltungswege; die Faltung ist enthalpisch getrieben mit ausgeprägter kinetischer Beteiligung (engl. kinetic partitioning) aller Faltungswege. $c M Y C-1245$ zeigt ein unvorhergesehenes non-Arrhenius Verhalten. Diese Konformation hat eine ungewöhnliche Schleifenanordnung (1:6:1), mit einer besonders langen internen Schleife. Das führt dazu, dass der Faltungsmechanismus bei höheren Temperaturen mehr dem Trichter-artigen Mechanismus der prädominanten Konformation gleicht; bei niedrigeren Temperaturen bilden sich zunehmend kinetische Fallen aus, die unter Energieaufwendung aufgebrochen werden müssen. Die Faltung der $c M Y C-1245$ Konformation ist daher ein seltener Grenzfall zwischen den beiden Regimen (rein entropisch, Trichter-artig vs. kinetic partitioning). Dieses non-Arrhenius Verhalten deutet auf eine Faltungsabfolge hin, die der von DNA/RNAHaarnadelstrukturen ähnelt. Die Ergebnisse verdeutlichen daher die komplexen und multiplen Faltungsmechanismen für G4. Die flachen konformationellen Energieoberflächen bzw. Energielandschaften führen dazu, dass unterschiedliche Faltungswege genutzt werden können und, dass sich eine Vielzahl unterschiedlicher Konformationen abseits eines globalen thermodynamischen Minimums ausbilden kann.

Um die Dynamiken zwischen den lokalen Minima der alternativen Konformationen ( $c M Y C-1234$ und $c M Y C-1245)$ und der thermodynamisch begünstigten Hauptkonformation cMYC-2345 zu untersuchen, wurde die bereits für G-Register Isomere genutzte Photocage-Strategie angewandt. Durch positionsspezifische Anbringung nur einzelner Photocages, konnten einzelne G-Strangabschnitte blockiert werden. Der Ansatz war erfolgreich, um die einzelnen, vollständig gefalteten Konformationen $c M Y C-1234$ und $c M Y C-1245$ in einer meta-stabilen Weise zu isolieren. Direkt nach der Photolyse-Reaktion ergibt sich dadurch ein Zustand, in dem ein vollkommen unmodifiziertes DNA-Oligonukleotid mit der natürlichen Sequenz des cMYC G4 in einer gefalteten Nicht-Gleichgewichtssituation vorliegt. Die exklusive Population dieser Faltungszustände unter nativen, physiologischen Bedingungen konnte so auf einzigartige Weise demonstriert werden. Beide Konformationen falten sich über mehrere Stunden hinweg zur Hauptkonformation um. Die Umfaltungskinetiken der beiden Konformationen weichen dabei stark voneinander ab, bei vergleichbarer Aktivierungsenergie für diesen Umfaltungsprozess. Die vergleichbaren Aktivierungsenergien weisen erneut darauf hin, dass der gleiche geschwindigkeitsbestimmende Schritt, nämlich die Brechung der Wasserstoffbrückenbindungen einer der G-Strangabschnitte, 


\subsection{Zusammenfassung}

zugrunde liegt. Die unterschiedlichen Umfaltungskinetiken, sowie der Vergleich zur Umfaltung der cMYC und hTERT G-Register Isomere, erlauben eine Skizzierung des Übergangszustands. cMYC-1234 gleicht dem Umfaltungsmechanismus der G-Register Verschiebung; es wird nur einer der G-Strangabschnitte aufgebrochen und ausgetauscht. $c M Y C-1245$ hingegen gleicht einem Umfaltungsmechanismus, bei dem ein deutlich höherer Entfaltungsgrad notwendig ist. Die langsamere Umfaltungskinetik lässt sich dadurch erklären, dass sich ein teilentfalteter Zustand neu orientieren muss, um in die Hauptkonformation übergehen zu können. Da die Rückfaltung des Ausgangzustands jedoch vermutlich hinreichend schnell verläuft, ist diese Konformation primär kinetisch gefangen.

Zusammenfassend kann festgestellt werden, dass es insgesamt erstmalig gelungen ist, die Kinetiken der wesentlichen Faltungs- und Umfaltungswege entlang der konformationellen Energielandschaft des cMYC G4 Elements zu untersuchen. Das komplexe, dynamische Zusammenspiel aller relevanten, nicht-konventionellen isomeren G4 Strukturen konnte entworren und umfassend experimentell beschrieben werden. Der dafür weiterentwickelte Ansatz über konformationelle Selektion mit Hilfe photolabiler Schutzgruppen hat dabei experimentelle Einblicke erlaubt, die bislang nicht zugänglich waren. Die Strukturen und Faltungszustämde, die mit den chemisch modifizierten Oligonukleotiden erhalten und isoliert wurden, sind umfassend spektroskopisch untersucht worden und konnten anhand der in der Literatur bekannten Strukturen verifiziert werden. Die Anwendung verschiedener spektroskopischer Ansätze und deren Kombination mit weiteren biophysikalischen Methoden hat eine Methoden-unabhängige Validierung der erhaltenen kinetischen und thermodynamischen Daten ermöglicht. 


\section{General Introduction}

\subsection{Structure and Dynamics of DNA G-Quadruplexes}

"DNA neither cares nor knows. DNA just is.

And we dance to its music."

Richard Dawkins, River out of Eden ${ }^{3}$

\subsubsection{Molecular Structure and Conformations of DNA}

$\underline{\text { Deoxyribonucleic }}$ acid (DNA) is now long known as the carrier of genetic information in all living organisms. ${ }^{4-6}$ The legendary discovery of its molecular structure by Franklin, Wilkins, Watson and Crick has been one of the major breakthroughs in modern science and laid the foundation for molecular biology in the way we understand it today. ${ }^{7-9}$ Though the architecture of DNA molecules up to the macroscopic level is of vast complexity, it can be disassembled to only four distinguishable primary building blocks. Figure 1 shows the DNA nucleotides/nucleobases adenosine/adenine (A), cytidine/cytosine $(\mathrm{C})$, guanosine/guanine $(\mathrm{G})$ and thymidine/thymine $(\mathrm{T})$ and their respective atom-wise numbering. All nucleobases are attached to a deoxyribose ring via an N-glycosidic bond. These nucleosides are linked via phosphodiester bonds and constitute the primary structure of a DNA oligo-/polymer.

Each of the nucleotides shows a specific hydrogen-donor/acceptor pattern, that allows interactions between nucleobases. In their structural model, Watson and Crick proposed the most stable interactions to be formed by purine-pyrimidine base pairs A-T and G-C (Figure 1). This arrangement leads to the formation of anti-parallel, helical double-strands with distinct structural parameters. The native, right-handed conformation of the DNA is known as B-form, since Rosalind Franklin found a dehydrated conformation, which she referred to as A-form. ${ }^{10}$ The DNA conformational alphabet was further extended with the discovery of a rare $\mathrm{C}$-form ${ }^{11}\left(\mathrm{Li}^{+}\right.$-DNA) and the left-handed Z-form ${ }^{12,13}$ as deviating helical conformations.

In a first approximation, this seems to be the end of the story - in terms of molecular structure; the complexity of structures for genomic DNA is less diverse compared to the cognate ribonucleic acid (RNA). Basically, genomic DNA is in situ synthesized as a double strand and thus born in its thermodynamic most stable conformation. Other than that, most of the RNA molecules usually appear as single stranded oligomers, which allows entering plenty of folding pathways along the energy landscape. In this regard, RNA is by nature much more prone to form different secondary structures such as stem-loops and hairpins and complex tertiary structures. ${ }^{14-18}$ 

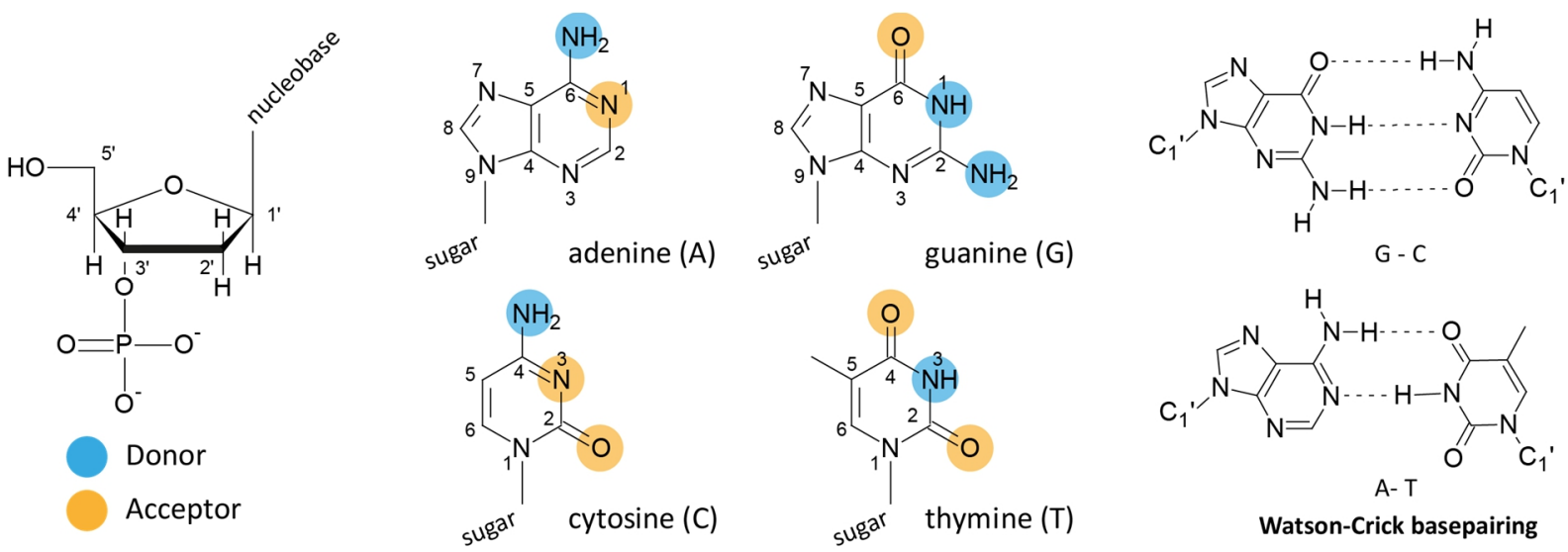

Watson-Crick basepairing

Figure 1: Nucleotides as basic building blocks of DNA: 2'-deoxyribose with 3'-phospate and 5'-OH group that allow polymerization via phosphodiester bonds (left). Purine (adenine, guanine) and pyrimidine nucleobases (cytosine, thymine) (middle) are attached via 1'- $\mathrm{N}^{1 / 9}$-gylcosidic bonds. Donor (blue)/acceptor (orange) patterns at the WatsonCrick interface are indicated. Watson-Crick base pairs between purine-pyrimidine nucleobases (A-T and G-C, right)

For single stranded DNA oligomers, the game changes and many secondary structures become possible, similar to those known for RNA. Many DNA oligomer sequences have been

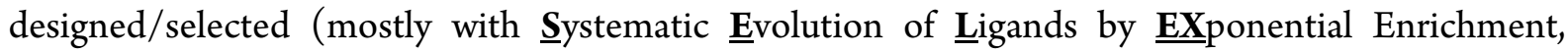
SELEX) $)^{19-21}$ especially to obtain certain structures of all kind or structure-related functions such as DNA hairpins ${ }^{22}$, DNAzymes ${ }^{23-26}$ or DNA-Aptamers ${ }^{27-29}$. However, some non-B-form DNA conformations (Figure 7) are also found naturally e.g. three-way junctions ${ }^{30-33}$, Holliday junctions ${ }^{34,3536}$, cruciform DNA ${ }^{37-40}$, hairpin DNA ${ }^{41,42}$ or looped DNA (D-, R-, T-loops) ${ }^{16,43}$.
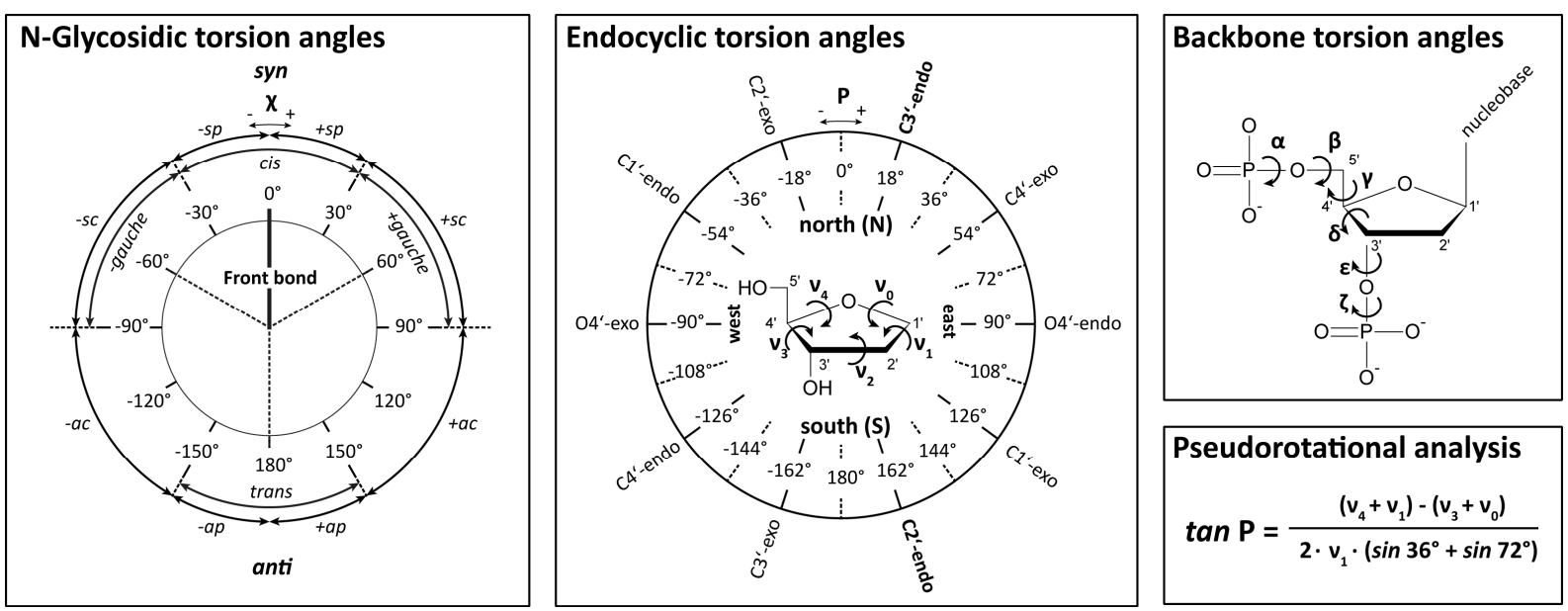

Figure 2: Nomenclature and definition of the torsion angles for N-glycosidic bond ( $\boldsymbol{\chi}$ ) rotation (left, Newman projection), endocyclic pseudorotation/(sugar pucker (middle) and backbone rotation (upper right). Figures adapted according to cited refernces. ${ }^{44,45}$ Abbreviations for Klyne-Prelog notation: $s p=$ synperiplanar, $s c=$ synclinal, $a c=$ anticlinal, $a p=$ antiperiplanar. $\mathbf{P}$ is defined as pseudorotational angle (lower right).

Figure 2 shows an overview for the parameters that determine the DNA conformation depending on the rotation or pseudorotation about given torsion angles between the ribose and the nucleobase, within the ribose ring and along the DNA backbone. The thermodynamic most favourable conformations for the $\mathrm{N}$-glycosidic bond angle (syn/anti) and the sugar pucker (2'-endo/3'-endo) are shown in Figure 3. 

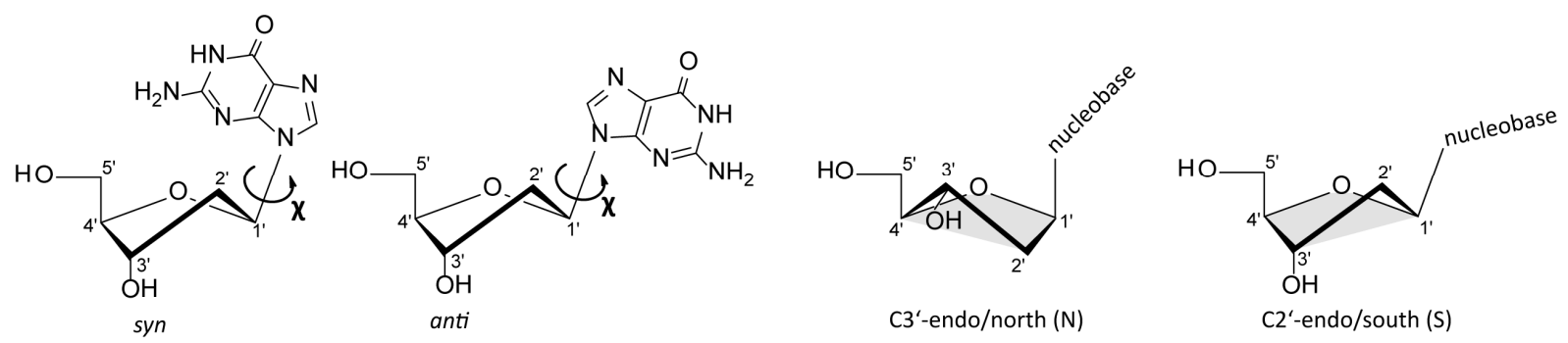

Figure 3: Preferred N-glycosidic conformations: $\operatorname{syn}\left(0<\boldsymbol{\chi}< \pm 90^{\circ}\right)$ and anti $\left( \pm 90^{\circ}<\boldsymbol{\chi}<180^{\circ}\right)$, (left); and preferred sugar pucker 3'-endo (N) or 2'-endo (S), (right). B-DNA adopts the C2'-endo/(S) conformation, while A-DNA adopts the $\mathrm{C} 3$ '-endo/(N) conformation. In duplex DNA N-glycosidic torsion angles are all anti. The sugar pucker affects the orientation of the nucleobase relative to the phosphate backbone.

\subsubsection{Non-Canonical DNA Structures}

Certain alignments of these parameters and the phosphate backbone make other interaction sites at the nucleobases accessible and alternative, non-canonical base pairing patterns become possible. ${ }^{46,47}$ Hoogsteen hydrogen bonding ${ }^{48}$ is of special importance and creates an own set of possible base pairs (Figure 4) that can also form in canonical duplex DNA. ${ }^{49}$ In regions with homo-purine (Pu) and homopyrimidine (Py) strands, the formation of different non-canonical paranemic DNA structures have been discussed. ${ }^{50,51}$ In mirrored tandem repeats one Py-strand can fold back (3'-Py: H-y3 or 5'-Py: $\mathrm{H}-\mathrm{y} 5)^{52}$ and form an intramolecular triplex via Hoogsteen interactions, known as H-DNA (Figure 7)..$^{39,52-57}$

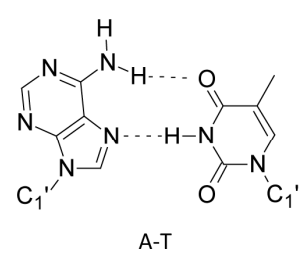

A-T

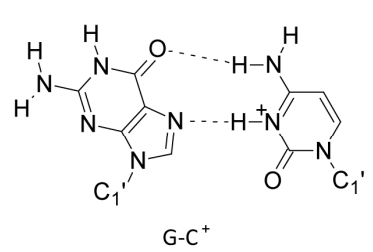

$\mathrm{G}-\mathrm{C}^{+}$

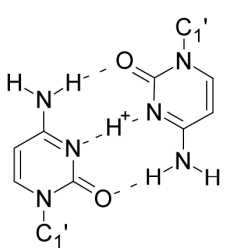

$c-c^{+}$

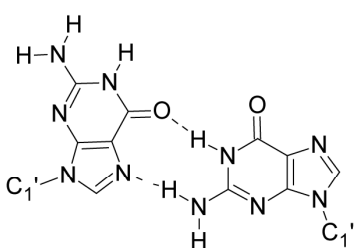

G-G

Figure 4: Hoogsteen base pairs between purine-pyrimidine (A-T and $\left.\mathrm{G}-\mathrm{C}^{+}\right)$, pyrimidine-pyrimidine $\left(\mathrm{C}-\mathrm{C}^{+}\right)$and purinepurine (G-G) nucleobases. The formation of hemiprotonated base pairs that involve $\mathrm{C}^{+}$is $\mathrm{pH}$-dependent $\left(\mathrm{pK}_{\mathrm{a}}(\mathrm{N} 3)=4.58\right.$, isolated cytosine) ${ }^{58}$

Already back in 1962 Gellert et al. ${ }^{59}$ could show that GMP in presence of monovalent cations forms planar G-tetrads via Hoogsteen interactions. In G-rich DNA sequences the planarity of the G-tetrads helps to stack upon each other stabilized by $\pi$ - $\pi$-interactions to form structures known as G-quadruplexes (G4), first reported by Sen and Gilbert in the late '80s. ${ }^{60,61}$ The recruitment of monovalent cations is a pre-requisite for the stability of this G4 structure, due to electrostatical reasons (Figure 5, Figure 7). ${ }^{62-65}$ Evidence that these DNA G4-structures really do form in vivo under cellular conditions is undisputed. ${ }^{66-68}$ The strong evidence for in vivo RNA G4 formation ${ }^{69-71}$ however is still controversially discussed. ${ }^{72}$ 

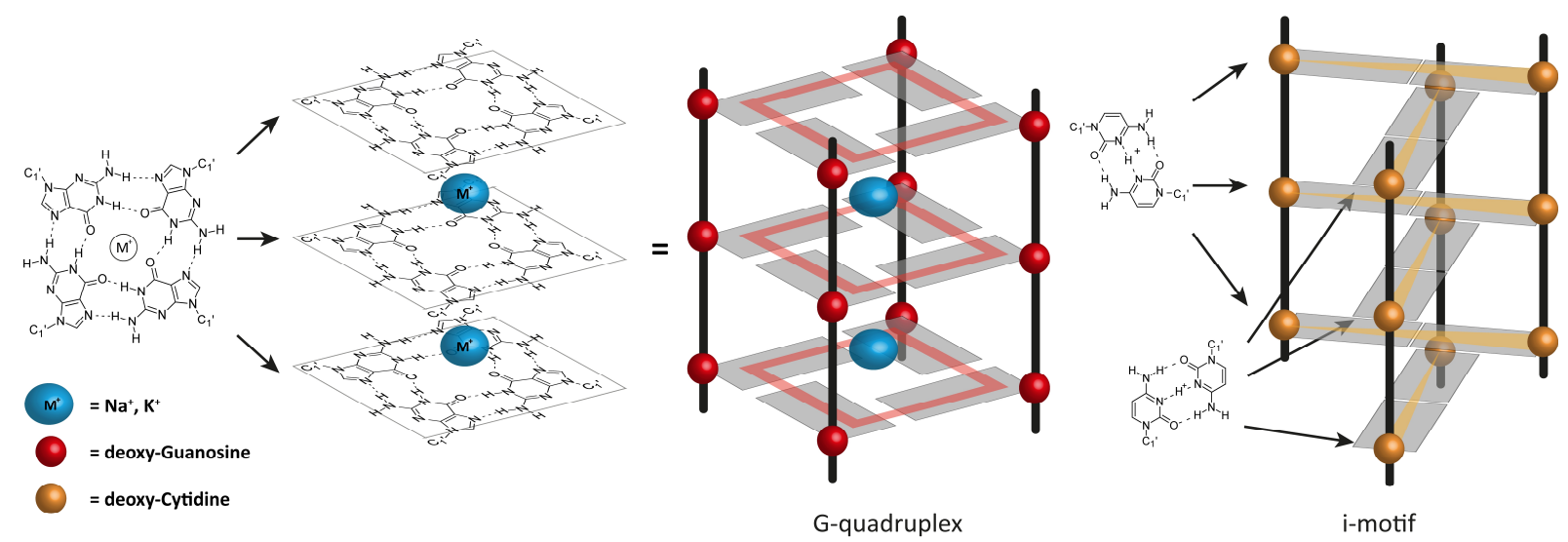

Figure 5: Three G-tetrads form a G-quadruplex (G4) structure via $\pi$ - $\pi$ stacking and stabilization with monovalent cations (mainly $\mathrm{Na}^{+}$or $\mathrm{K}^{+}$), (left). Schematic representations of four-stranded DNA structures (right) nucleotides are represented as coloured spheres with planar nucleobases; hydrogen-bond connectivities are indicated.

Most comparable to G4s, Cytidines can form four-stranded structures, known as i-motif. ${ }^{73-82}$ The overall structure ${ }^{83}$ is build up by stepwise alternating diagonal hemi-protonated $C^{+}-C$ base pairs. The base pairs are stacked with offset, so that from a top view each $2+2$ crossing base pairs define a jagged tetrad (Figure 5, Figure 7). DNA i-motif structures have also been detected in human cells. ${ }^{84}$

Canonical B-form DNA is by far the most important non-regulatory conformation, since it is the conformation of chromosomal DNA that is adopted to guarantee stability for the genes. For gene regulation and additional functionality in gene expression G-quadruplexes have now emerged as most important non-B-form DNA conformation. This is highlighted by (December 2020) more than 8731 publications in the Web of Science (Clarivate Analytics; Figure 6, left), increasing relative share of publications in the nucleic acid field of research (Figure 6, right) and more than 421 structures in the Protein Data Bank (RCSB PDB, “G-quadruplex”).
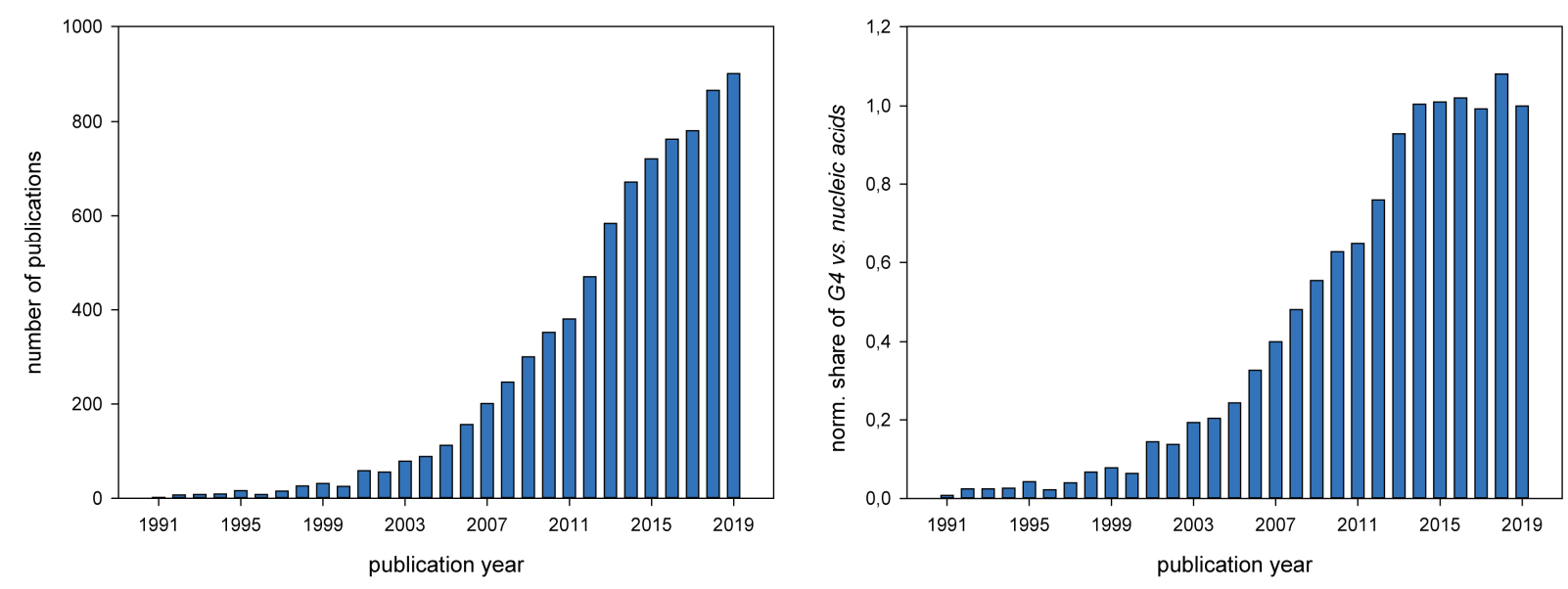

Figure 6: Number of G-quadruplex related publications from 1991-2019 (“G-quadruplex"). Left: total numbers, right: in relation to the total number of nucleic acid publications ("nucleic acids, or DNA, or RNA", normalized share). 

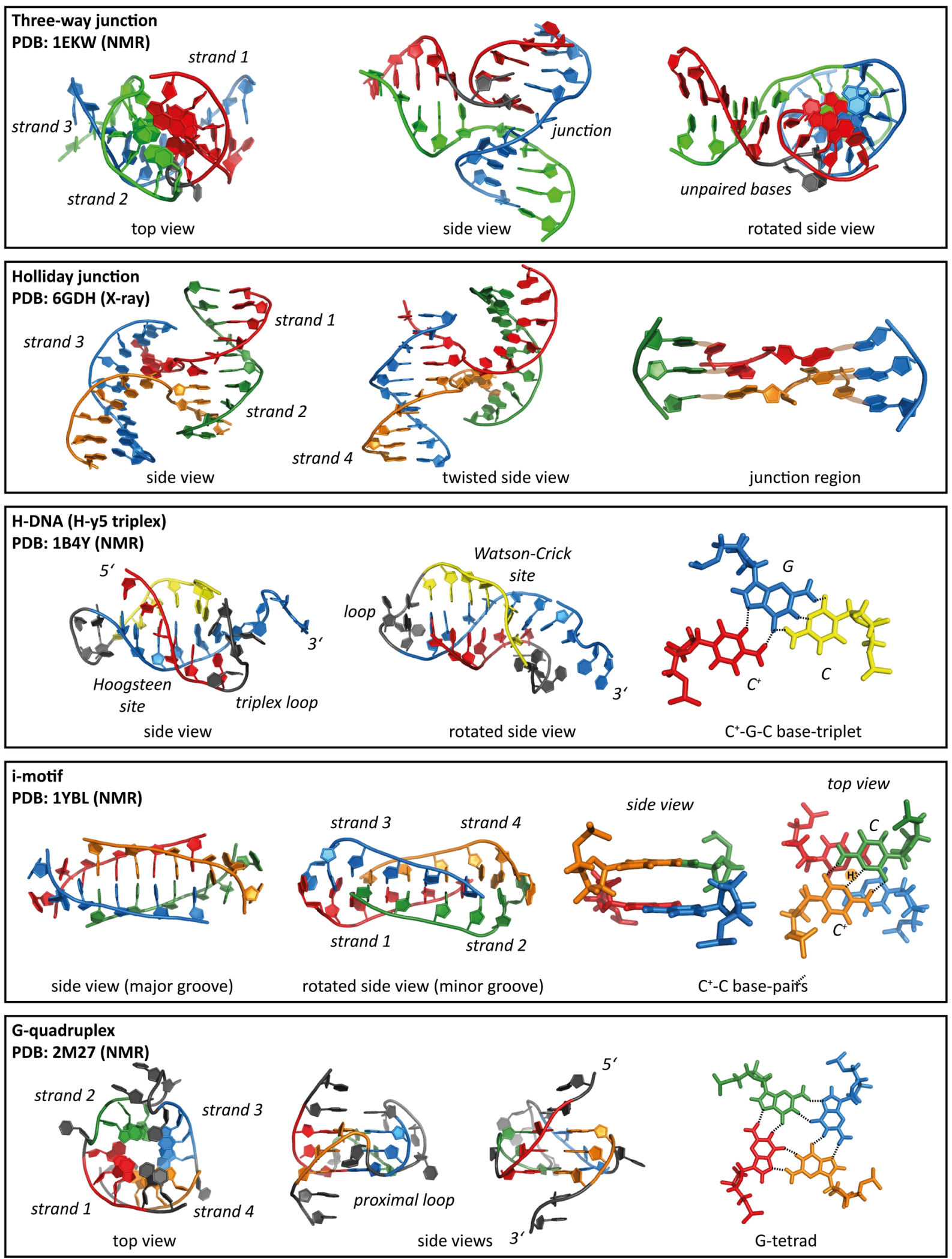

Figure 7: Overview of non-canonical (non-B-form) DNA structures. Representative PDB-structures are shown for threeway junctions (PDB: 1EKW) ${ }^{30}$, four-way junctions (Holliday junction, PDB: 6GDH) ${ }^{36}$, triplex DNA (H-DNA, PDB: $1 \mathrm{~B} 4 \mathrm{Y})^{52}$ and tetraplex DNA (i-motif, PDB: $1 \mathrm{YBL}^{75}$; G-quadruplex, PDB: $2 \mathrm{M} 27^{85}$ ). 


\subsubsection{Canonical Structural Polymorphism}

"Folding" of nucleic acids in general, describes the process of formation of secondary and tertiary interactions that define the structure of the macromolecule. A folded state can thus be defined as a state that represents a thermodynamic minimum on the energy landscape. This state can still be highly dynamic and is not necessarily the most stable state, but in principle, it can be described with defined coordinates. In the context of G-quadruplexes, folding usually refers to the topology of a specific conformation. In this way, the directionality of the DNA-backbone and the relative orientation of the four strands to each other defines the G-quadruplex fold. G-quadruplexes can be formed both interand intramolecular which results in tetra-, bi- or unimolecular G-quadruplexes. Irrespective of their molecularity, herein, a G-quadruplex is defined as a four-stranded structure, referring to the four G-tracts that constitute the G-quadruplex core structure. These strands can be oriented in an all-parallel, $(3+1)$ or hybrid 2+2) anti-parallel conformation, which force different nucleobase conformations and tetrad polarities (Figure 8); in the case of uni-/intramolecular G-quadruplexes, the linking loop sequences are forced in distinct arrangements. ${ }^{86}$ The structural key features depicted in Figure 8 divide the conformational landscape in fundamentally separated subsets of conformations. These canonical G4 structures are in part predictable from sequence. ${ }^{87}$

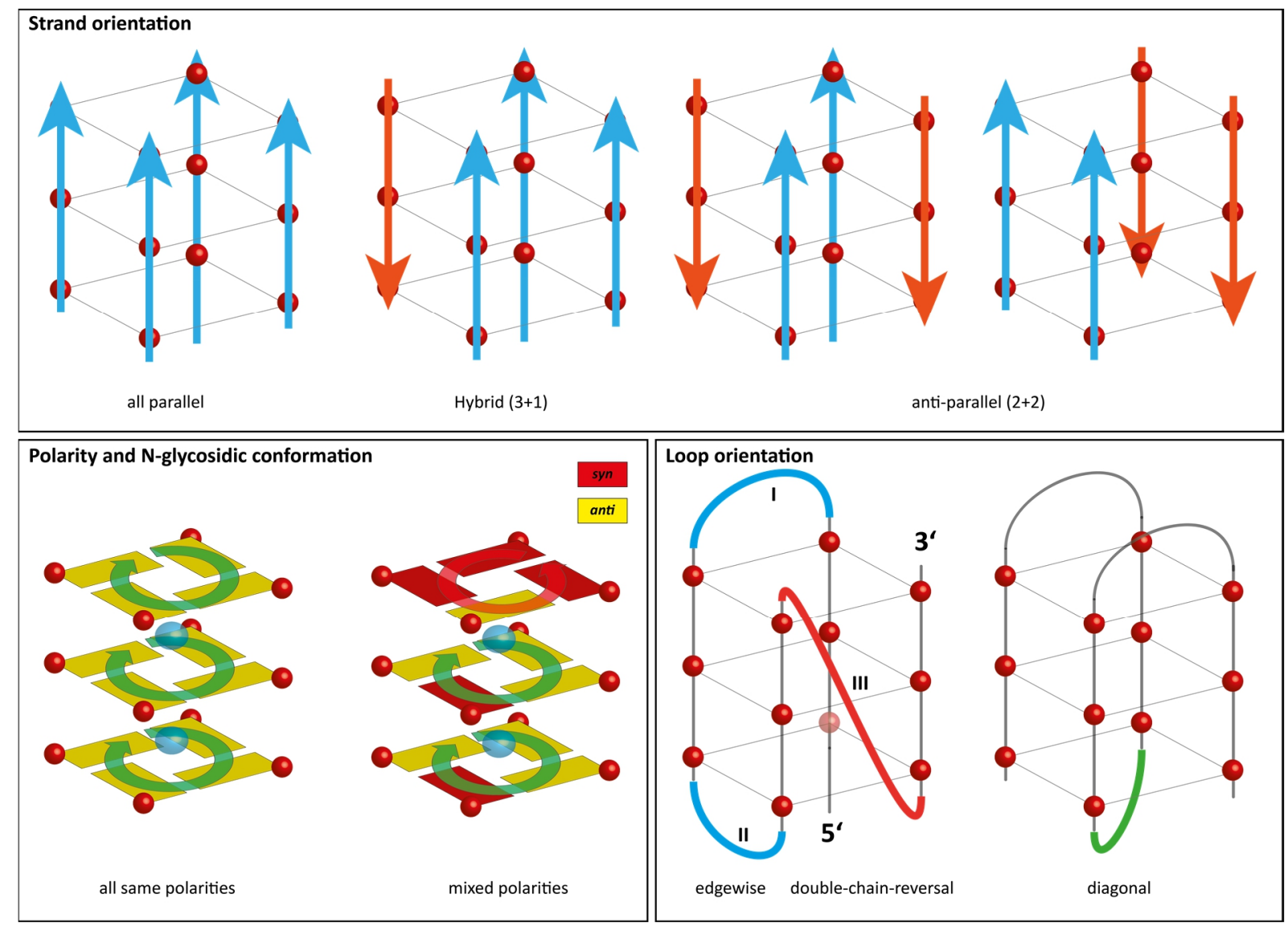

Figure 8: Canonical structural features that determine a G4 structure: strand orientation/directionality (relative orientation shown in orange/blue), tetrad polarity and $\mathrm{N}$-glycosidic conformation (all-parallel G4s are in all-anti conformation) and loop orientation. The three constituting loops are numbered in 5' -3 ' direction. 


\subsection{Structure and Dynamics of DNA G-Quadruplexes}

The depicted $(\mathrm{n}=3)$-layered motifs are the most common G4-motif. In general however G4s with $\mathrm{n} \geq 2^{88}$ layers can form stable structures, depending on the nucleotide sequence $\left(\mathrm{n}=2^{89-92}, \mathrm{n}=4^{93,94}\right)$.

\subsubsection{Non-Canonical Structural Polymorphism}

Besides the canonical structural features that define the G-quadruplex structure in terms of folding topology, sugar conformation and loop orientation, additional non-canonical features add layers of complexity to the structural polymorphism. ${ }^{95-98}$ Especially along G-rich sequences that do not fit the consensus G-quadruplex sequence (2.3.1) additional structural isomerism can occur (Figure 9), which has broadened the definition of G4 structural parameters.

G-tracts with more than $\boldsymbol{n}(\mathrm{n}+\mathrm{x}$, with $\mathrm{n}=$ number of tetrads in the folded G4) subsequent G-residues can be shifted relative to the tetrads to incorporate different G-residues into the G4-core (G-register shift). This kind of isomerism is possible for many reported G4 sequences (e.g. $c M Y C^{99-101}, V^{2} G F^{85,101}$ and $\left.h T E R T^{102,103}\right)$. Poly-G stretches of up to 30 nucleotides found e.g. in C. elegans ${ }^{104,105}$ have been shown to adopt distinct parallel G4 structures. ${ }^{106}$ Stable G4 structures with n-1 G-tracts have been reported recently. Here, the G4-core lacks a single position in a tetrad, but the remaining triad is stable enough to maintain a stable G4 structure. ${ }^{107,108}$ This G-vacancy sites can be filled up with guanine metabolites which mimics an ideal binding pocket with specific molecular recognition. ${ }^{109,110}$

If more than four G-stretches are nearby in a DNA sequence, also different G-tracts can be incorporated into the G4 structure. This feature is known as spare-tire isomerism and also has been reported for many $\mathrm{G} 4$ sequences $\left(c M Y C^{111-113}, V E G F^{113}\right)$. If the number of $\mathrm{G}$-tracts increases in longer G-rich sequences, multiple, stacked ${ }^{114} \mathrm{G}$-quadruplexes can be formed or potentially switch between hairpin-G-quadruplex arrangements. This has been examined especially for the hTERT core promoter. ${ }^{102,103,115-117}$ Further increasing the number of G-tract repeats can result in the formation of multimers, ${ }^{118}$ called G-wires. ${ }^{119,120}$ These nanostructures have been visualised with atomic force microscopy (AFM) in Tetrahymena telomeres with $\mathrm{G}_{4} \mathrm{~T}_{2} \mathrm{G}_{4}$ repeats. ${ }^{121}$ The concatenation of stacked G-quadruplexes can be achieved in different ways and results in highly polymorphic types of polymer structures.

Finally, peculiar loop and strand arrangements yield non-canonical structural features like bulges ${ }^{122-125}$, hairpin loops ${ }^{117,126}$, hairpin loops within a bulge ${ }^{127}$ or snap-back motifs ${ }^{91,128-131}$. All of the diverse canonical and non-canonical G-quadruplex structures share a high similarity in the overall G4-core constitution. However, the schematic representation neglects the fact that G4s are indeed helical structures. Typically they are right-handed, but more recently structures of left-handed G4s have been reported (Z-G4). ${ }^{132-135}$ 
The group of J. Plavec has reported on a series of novel tetrahelical structures from tandem repeats of alternating GGG and GCG tracts with non-canonical G-A and G-G base pairs as well as from AGCGA-rich DNA with GAGA- and GCGC-quartets. ${ }^{136-139}$ They also characterized a G-quadruplex structure from $\mathrm{G}_{4} \mathrm{C}_{2}$ repeats that features different stacked C-C base pairs. ${ }^{94}$ The formation of $\mathrm{G} 4 \mathrm{~s}$ in expanded $\mathrm{G}_{4} \mathrm{C}_{2}$ hexanucleotide repeats in the $\mathrm{C} 9$ orf 72 gene is strongly linked to neurodegenerative diseases as amyotrophic lateral sclerosis (ALS) and frontotemporal dementia (FTD). ${ }^{140,141}$
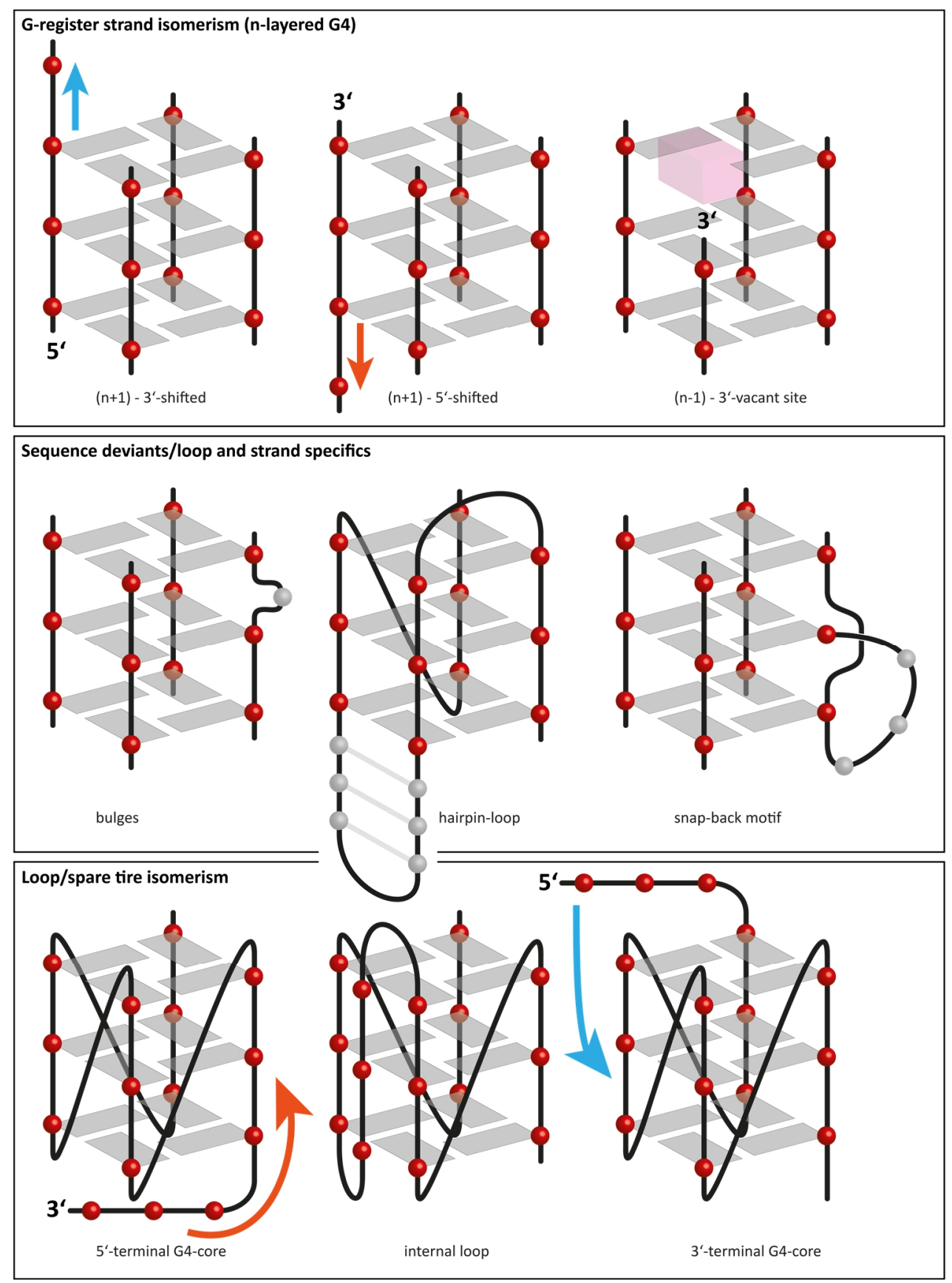

Figure 9: Non-canonical structural features that broaden the regular conformational space of G4 structures. Upper part: G-stretches that have $(n+x)$ G-nucleotides can form $(x+1)$ n-layered isomers by formally shifting the G-registers; (n-1)-G-stretches can form G4s with G-vacancy sites in the respective G-tetrad. Middle: rare structural elements that can be found in G4 motifs. Lower part: Loop isomerism in G4 forming sequences with 4+x G-stretches. 


\subsubsection{Conformational Dynamics}

The pronounced structural polymorphism is linked to inherent conformational dynamics (Figure 10). ${ }^{142}$ Refolding and topology exchange has been investigated for the human telomeric G4 $4^{143,144}$ as well as for the human telomerase promoter G4 (hTERT) ${ }^{102,145} \cdot{ }^{146}$ After initial folding in two different hybrid conformations (see also chapter 2.2.3), the telomeric G4s refold via an ensemble of partially unfolded states into the conformational equilibrium. ${ }^{144}$ The partially unfolded states remain as long-lived intermediates for several hours. The hTERT G4 co-exists in two conformations, a hybrid and a parallel. The parallel conformation is populated nearly exclusively at higher temperatures, while at ambient temperatures the hybrid conformation is slightly more populated (60\%). Nußbaumer et al. ${ }^{145}$ inserted $8-{ }^{13} \mathrm{C}$-guanosines into conformation-specific positions and monitored the relaxation back to the conformational equilibrium after a temperature-jump with BEST-TROSY ${ }^{1} \mathrm{H}-{ }^{13} \mathrm{C}-\mathrm{HSQC}$. They found a slow refolding rate for the interconversion of the two conformations $\left(0.72 \mathrm{~h}^{-1}, 90 \mathrm{mM} \mathrm{K}^{+}, 298 \mathrm{~K}\right)$.

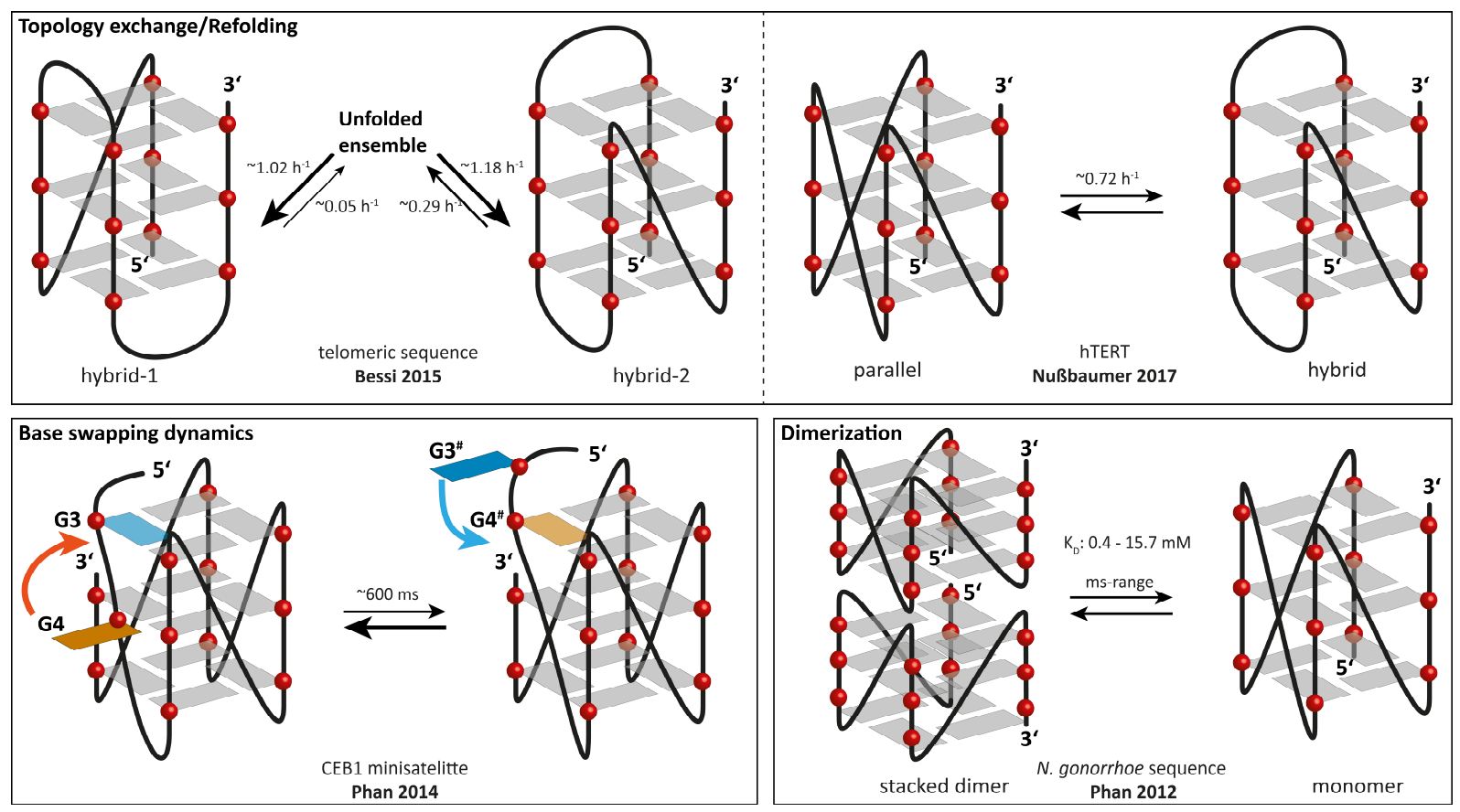

Figure 10: Different types of conformational transitions in folded G4s: topology exchange that involves complete refolding between two different conformations, base swapping dynamics of G-residues in a G-tetrad and dimerization dynamics between a stacked dimer and the folded monomer.

Phan et al.${ }^{91}$ have studied the conformational dynamics of a stacked dimeric G4 from the human CEB1 minisatellite. ${ }^{91}$ Using NOESY and ROESY experiments they found conformational exchange at the 5 -end in the millisecond timescale $(\sim 600 \mathrm{~ms})$. They propose a model for base swapping dynamics of two G-nucleotides that exchange in tetrad formation. Such guanine-flipping dynamics are involved in the unwinding mechanism of G4s by RecQ helicases. ${ }^{147}$ In another study, the group of A.T. Phan investigated the dynamics of a parallel conformation that dimerizes via tetrad stacking on the 5' -5 ' interface. ${ }^{114,148}$ The $\mathrm{K}_{\mathrm{D}}$ for the dimer formation is concentration dependent ([DNA] and $\left[\mathrm{K}^{+}\right]$) and sequence specific, but overall in the low mM-regime (approximately in the range of milliseconds). 
Loop dynamics of a CEB25 G4 structural ensemble have been analyzed in the sub- $\mu$ s regime with $\mathrm{MD}$ simulations using NMR RDC-restraints. ${ }^{149}$ Conformational dynamics that are associated to G-register exchange and spare-tire exchange have been described as a consequence of thermodynamic and biological considerations. ${ }^{101,113}$ However, interconversion dynamics for these non-canonical structural features remain largely elusive and will be investigated and discussed in detail as a result of this thesis. The main thermodynamic parameters that have been reported so far on dynamics of G-register isomers are summarized in the following section. The observation of thermal hysteresis in the melting and annealing curves for an ensemble of G-register isomers in the $c M Y C$ G4 forming sequence (see chapter 2.4) allowed an indirect approximation of their exchange rates. ${ }^{100,101}$ Figure 11 shows an overview of the main parameters $\left(\Delta \mathrm{G}_{25}, \mathrm{~K}_{\mathrm{ex}}\right.$ and $\Delta \mathrm{H}$ reported at $5 \mathrm{mM}\left[\mathrm{K}^{+}\right], \mathrm{E}_{\mathrm{a}}$ at $\left.2 \mathrm{mM}\left[\mathrm{K}^{+}\right]\right)$. Chapter 4 of this thesis presents experimental results for G-register exchange dynamics in the context of promoter G4 sequences (in particular $c M Y C$ ).

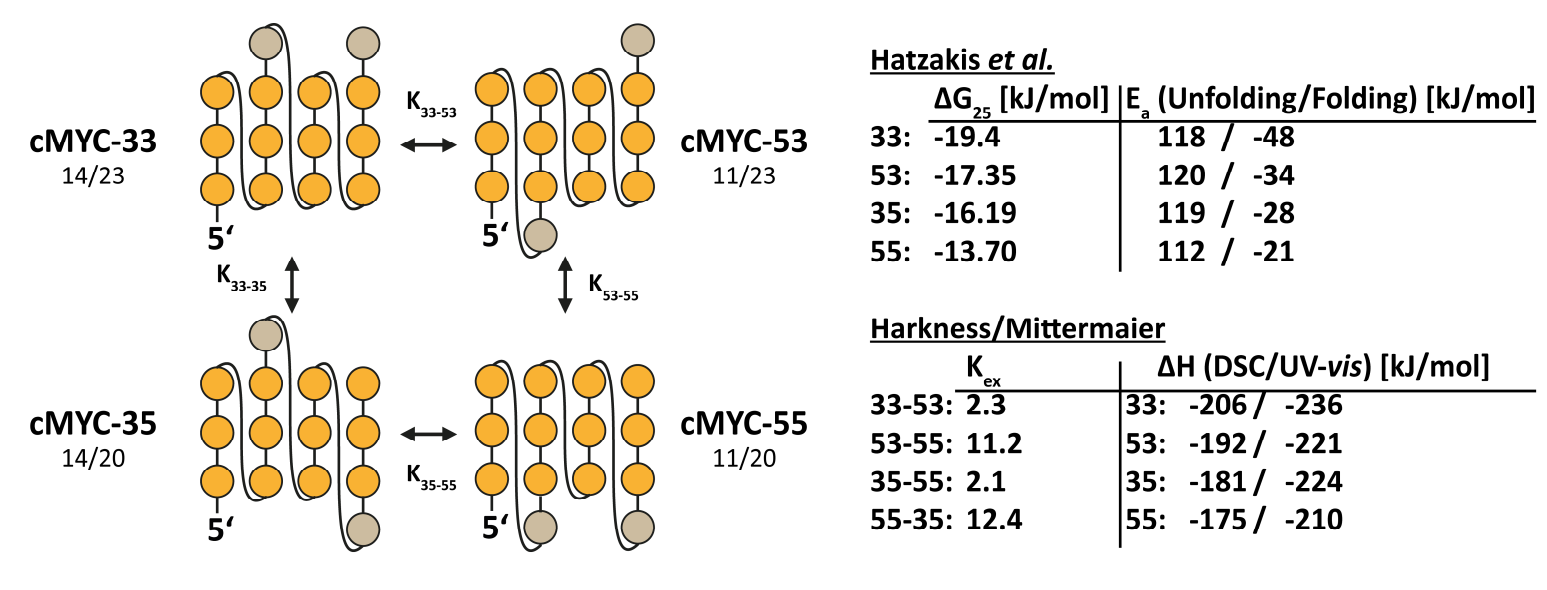

Figure 11: Left: schematic representation of possible G-register isomers in the cMYC G4 (adapted from Harkness and Mittermaier ${ }^{101}$ ). The numbering shows both the G-register nomenclature by Harkness and Mittermaier used throughout this thesis and their equivalent by Hatzakis et al. ${ }^{100}$. Right: Summary of the main experimental findings for $\Delta \mathrm{G}_{25}, \mathrm{~K}_{\mathrm{ex}}, \Delta \mathrm{H}$ $\left(5 \mathrm{mM}\left[\mathrm{K}^{+}\right]\right)$and $\mathrm{E}_{\mathrm{a}}\left(2 \mathrm{mM}\left[\mathrm{K}^{+}\right]\right)$.

Immediately prior to the submission of this thesis, Mittermaier et al. published a paper that outlines the consequences of an increasing length of telomeric repeat (TTAGGG) ${ }_{n}$ sequences with increasing number of adjacent G-tract. ${ }^{150}$ They describe a thermodynamic and kinetic folding frustration that arises from a competitive incorporation of G-tracts into contiguous G4s with negative cooperativity. The simulated folding frustration for a 32-repeat sequence $(n=32)$ is based on global analysis of rapid thermal melting experiments with thermal hysteresis (as also discussed above). The frustrated folding energy landscape results in partially unfolded and potentially misfolded parts of the DNA chain. This observation has implications for possible related conformational dynamics that would be required to straighten up the entangled G4 chain. It is conceivable that this process involves a step-by-step sparetire exchange refolding that evolves through the DNA chain. Chapter 5 of this thesis presents experimental results for spare-tire exchange dynamics in the context of promoter G4 sequences. 


\title{
2.2 Folding Dynamics of DNA G-Quadruplexes
}

\author{
"Thus, a pathway offolding means \\ that there exist a well-defined sequence of events \\ which follow one another $[\ldots] "$
}

Cyrus Levinthal, How to fold graciously ${ }^{151,152}$

\subsubsection{Folding Energy Landscapes and Folding Pathways}

The observation that protein folding is faster than hypothesized from theoretical considerations (Levinthal's paradoxon) led to the proposal of a folding funnel. ${ }^{1,151,152}$ In the funnel-like folding energy landscapes of proteins, a deep well represents the native state of a folded protein (Figure 12). The fast and apparently barrier-free folding of proteins seems contradictory, given the complex nature of protein structures. ${ }^{153,154}$

However, it is exactly this structural complexity and the residue diversity (20 amino acids in proteins) that is now commonly assumed to guide an optimized folding. Thus, the native structure of proteins is likely to be pre-defined by its primary sequence (Anfinsen's dogma). ${ }^{155,156}$ Following this, folding energy landscapes of non-canonical DNA structures must certainly be more complex, because only four residues (in G-quadruplexes only one) determine the specificity of local contacts. G-quadruplex forming oligonucleotides are built from G-rich sequences; hence, the poor nucleotide dispersion is self-defining. ${ }^{157}$

Indeed, folding of nucleic acids is fundamentally different and folding of DNA G-quadruplexes in general is a slower process compared to proteins. The folding energy landscapes of nucleic acid oligonucleotides are not funnel-like but rough with flatter wells (Figure 12). In particular, the folding energy landscapes of DNA G-quadruplexes are complex, with numerous competing basins of attraction. This results in a pronounced structural polymorphism, the coexistence of different folded states and different competing folding pathways. Thus, in many cases it is not appropriate to speak about "native states", since many nucleic acid sequences are prone to adopt several competing native folds. ${ }^{157}$

The coexistence of different folded states is a clear sign of a kinetic partitioning folding mechanism for G-quadruplexes. However, even in the absence of concurrent conformations multiple folding pathways must be assumed. This follows from the consideration that no restraints do apply for the conformational space during folding that would limit the complexity of possible sub-states. Some intermediate states are long-lived and give clear implications for kinetic traps during folding; others are short lived and have not been detected directly in experimental studies. ${ }^{157}$ 

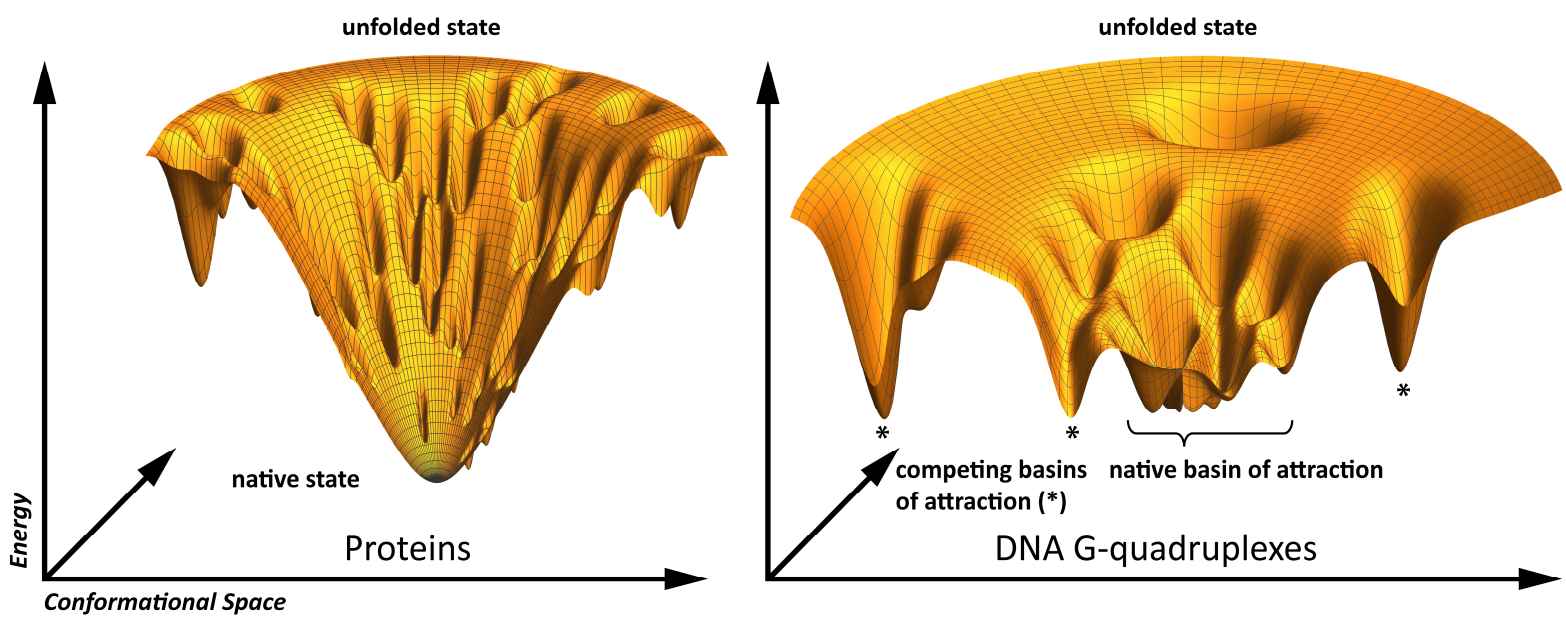

Figure 12: Representation of folding energy landscapes. Left: Folding-funnel as it is commonly proposed for protein folding. Right: Folding energy landscape with flat wells and competing basins of attraction, proposed for G-quadruplex folding. It is commonly assumed that proteins and nucleic acids follow folding pathways along their energy landscapes. Folding dynamics are typically described as funnel-like (left, proteins) or with kinetic partitioning (right, oligonucleotides).

Here, it must be noted that the definition of unfolded, partially folded, folded and misfolded states is an arbitrary definition, since any given oligonucleotide orientation is just a Boltzmann distributed ensemble over the available conformational space under given conditions at thermodynamic equilibrium. One has to be aware that any folding or refolding process is affected by external conditions and the physical nature of its starting point. This holds especially true for ensembles of unfolded states that can be prepared in different ways and lack a clear definition. ${ }^{157-160}$ From an energetic perspective, thermal denaturation marks a complex ensemble of high-entropy states, while e.g. force unfolding yields low-entropy states. The complexity of unfolded states can also be visualized easily with the synand anti-patterns of G4 forming oligonucleotides. In an unfolded oligonucleotide, every G-residue can adopt either of the two glycosidic conformations, which sums to $2^{12}=4096$ combinations in a 3-layered G4. If a certain combination is productive or not, meaning if it is on-or off- the folding pathway, depends on the thermodynamic stability of the finally folded conformation. Hence, a description of the entire folding process is a seemingly impossible task for computational methods, as the starting point in these simulations is hard to define. Nevertheless, both experimental and theoretical studies have proposed numerous folding intermediates. ${ }^{161-163}$ Again, it depends on the underlying definition, if those are off- or on-pathway.

Recent progress in computational studies and $\mathrm{MD}$ simulations has helped to describe and outline the conformational energy landscapes and trajectories of DNA G4 with major contributions from the group of J. Šponer. ${ }^{157,161-166}$ Experimental studies to investigate G4 folding have been published using mass spectrometry ${ }^{167,168}$, CD-spectroscopy ${ }^{169-171}$, NMR-spectroscopy ${ }^{144,172}, \operatorname{smFRET~microscopy~}{ }^{173,174}$ and force spectroscopy with magnetic tweezers ${ }^{175,176}$. 


\subsubsection{Experimental Approaches to Measure Folding Kinetics}

Many experimental approaches to investigate folding kinetics of G-quadruplexes rely on rapid mixing with monovalent cations to induce isothermal folding. ${ }^{177}$ Otherwise, thermal denaturation allows investigating folding processes under otherwise physiological conditions. Kinetic information on (un)folding events of G-quadruplexes can be obtained from thermal hysteresis, when samples are rapidly heated or cooled. ${ }^{100,178,179}$ Isothermal unfolding kinetics as a transition from G4 to duplex DNA can be investigated by addition of the complementary strand (complement trapping). ${ }^{170,180-182}$ A method that allows investigating (un-)folding kinetics both isothermally and in the presence of monovalent cations is mechanical unwinding. This has been demonstrated with force microscopy on oligonucleotides that are attached to magnetic tweezers. ${ }^{176}$

However, results from different folding experiments have a limited comparability (see Table 1 for overview). The reason for this is the lack of a clear definition for an unfolded state. Obviously, the nature of an unfolded state can be drastically different: (i) Thermal denaturation or high-pressure unfolding represent the population of thermodynamically excited states. ${ }^{183,184}$ (ii) Chemically unfolded states in the absence of monovalent cations or due to $\mathrm{pH}$ changes can alter the conformational energy landscape itself. ${ }^{159,160}$ (iii) Unfolding with external mechanical tension restricts the flexibility of oligonucleotides and constrains predefined orientations. ${ }^{175,176,185}$

While mechanical unfolding ensures complete unfolding in a linearly stretched strand, chemically altered conformational energy landscapes can cause the population of alternative folds like hairpins or pre-folded states. ${ }^{158-160,186,187}$ In all cases, the experimental conditions determine different starting points for folding pathways that result in different folded conformations. Furthermore, oligonucleotides have significantly greater flexibility at their termini than DNA sequences in a genomic context. A study on G-triplex formation has investigated the influence of proximal DNA and found increased complexity for the dynamics of tethered oligonucleotides. ${ }^{188}$ The DNA flanking regions also have an impact on the kinetics of anti-parallel vs. parallel topologies. ${ }^{188}$ This might be especially important for the evaluation of smFRET-derived kinetics, where often the dyes are attached at double stranded overhangs. ${ }^{189}$ The influence of different metal cations on the folding kinetics has also been studied in detail. ${ }^{190,191}$

Table 1: Comparison of different methods to induce folding of G4s. All methods modulate the folding energy landscape and thermodynamic aspects during folding in a different way.

\begin{tabular}{c|ccc} 
& constant $[\mathrm{K}+]$ & isothermal & flexibility/tumbling \\
\hline mixing/chemical & - & + & + \\
hysteresis/thermal & + & - & + \\
force/mechanical & + & + & -
\end{tabular}




\subsubsection{Folding Kinetics of DNA G-Quadruplexes}

The timescale of folding kinetics for DNA G-quadruplexes ranges from milliseconds to minutes. ${ }^{171,177}$ The folding behaviour is highly sequence dependent and is sensitive to ion-concentration and buffer conditions. ${ }^{191,192}$ For many G4 forming sequences, multi-pathway folding ${ }^{173}$ has been reported and parallel folding into different conformations has been observed. ${ }^{174}$ In general, folding of G4s is multiphasic and is supposed to involve intermediates like anti-parallel hairpins ${ }^{165,170,171}$, triplexes ${ }^{193-195}$ and (n-1)-tetrad conformations. ${ }^{167,196-198}$

Folding of the human telomeric sequence (TTAGGG) $)_{\mathrm{n}}$ and its sequence variants have been studied most extensively, both experimentally ${ }^{144,159,167,169,171,173-175,188,199-201}$ and theoretically ${ }^{202-204}$. The telomeric G4 adopts at least two different hybrid conformations under physiological conditions. ${ }^{205-210}$ $\mathrm{K}^{+}$-induced folding revealed a kinetic partitioning mechanism, where one hybrid conformation is kinetically favoured. The conformational equilibrium is reached only after days and partially unfolded states have been observed as long-lived intermediates. ${ }^{144}$ This highlights the importance of kinetic studies, since non-equilibrium G4 conformation can have lifetimes that exceed the biological relevant timescales ( $\sim 20 \mathrm{~ms} / \mathrm{nt}$ for DNA replication and $\sim 200 \mathrm{~ms} / \mathrm{nt}$ for transcription)..${ }^{171,211}$ This means that from a chemists point of view, there are kinetic and thermodynamic products of G4 folding. ${ }^{201}$

The kinetics of G-quadruplex folding are very sensitive to ionic conditions ([ $\left.\mathrm{K}^{+}\right]$in the following). In principle, studies at physiological conditions should be most reliable to evaluate kinetic aspects, which is however difficult for experimental reasons (2.2.2). Dimerization during folding is a critical aspect that can bias the folding kinetics at higher $\left[\mathrm{K}^{+}\right]{ }^{212}$ Kinetics of tetramolecular G-quadruplex assembly have been studied. ${ }^{213-215}$ In these intermolecular studies a stepwise mechanism was proposed with a fast monomer-dimer equilibrium followed by an intermediate triplex formation that seems to be stabilized especially at higher $\mathrm{K}^{+}$concentration. ${ }^{214}$ Folding pathways inspired by such stepwise strand recruitments are often transferred to explain G4 folding kinetics, but this has to be treated with caution, since the kinetics and thermodynamics of intermolecular assemblies are inherently different from intramolecular folding. At very low $\mathrm{K}^{+}$concentrations ( $<1 \mathrm{mM}$ or substoichiometric) a drastic effect on the kinetics is observed, since the recruitment of $\mathrm{K}^{+}$-ions gets rate-limiting and determines the overall folding pathways. ${ }^{167}$ These effects on folding acceleration saturate however at approx. 2-3 mM concentrations of $\mathrm{K}^{+},{ }^{177}$ whereas the thermal stability of G4s still increases at much higher $\left[\mathrm{K}^{+}\right] .{ }^{196}$

The geometry of the loops is a critical parameter for the thermal stability of G4s and determines the folding topologies that are preferentially adopted (2.1.2). ${ }^{216,217}$ The loops also have a considerable impact on the folding kinetics ${ }^{100,216}$ and can shift the folding process towards different pathways via prefolded arrangements. ${ }^{159,160,218}$ An effect on accelerated folding has been observed especially for hairpinforming loops. ${ }^{127,158,192,219}$ 


\subsubsection{Comparison to RNA G-Quadruplexes}

RNA G4s generally show reduced structural polymorphism. ${ }^{220,221}$ They tend to adopt all-parallel conformations, where all sugars are in anti-configuration. ${ }^{222,223}$ Chemical modifications like 8-bromoguanosine can be used to flip the glycosidic conformation and force RNA G4s into anti-parallel conformations. ${ }^{224,225}$ In line with that, folding kinetics of RNA G-quadruplexes are markedly different and are considered to be much faster compared to their corresponding DNA sequences. ${ }^{171,172}$ The human telomeric-repeat containing RNA (short: TERRA) ${ }^{226-231}$ sequence is one of a few examples that has been investigated in aspect of RNA folding and compared with its homologous DNA sequence. ${ }^{171,172}$ The drastically faster RNA-G4 folding has been explained with a reduced conformational space that results from pre-oriented sugar configurations. The more complex (and stable) syn-anti patterns in DNA G4s lead to an increased number of unproductive microstates and potentially misfolded conformations.

\subsubsection{Mutual Exclusive Formation of DNA i-motifs and G-Quadruplexes}

The folding of i-motifs and G-quadruplexes is a comparably complex process. The rapid $\mathrm{pH}$-induced folding of i-motifs has been investigated with time-resolved NMR and revealed a kinetic partitioning mechanism. ${ }^{76-78,80}$ Within this study ${ }^{76}$ it was shown that two coexisting i-motif conformations fold in parallel with a kinetically favoured conformation $\left(\sim 0.9 \mathrm{~min}^{-1} \mathrm{vs.} 2.0 \mathrm{~min}^{-1}\right)$. The subsequent refolding process to reach conformational equilibrium is slower by about two orders of magnitude.

Under ex vivo conditions, it was found that in many cases the formation of i-motifs and G-quadruplexes is mutually exclusive. ${ }^{232-234} \mathrm{~A}$ steric hindrance and differences in their different requirements for stability at physiological conditions impedes a simultaneous formation. ${ }^{235}$ However, the i-motif structure itself is highly dynamic. It can switch between hairpin and i-motif conformations in the C-rich strand, which affects the formation of G-quadruplexes in the complementary strand and the recognition by protein binding partners (such as hnRNP-LL). ${ }^{81,236-239}$ The influence of chemical and mechanical factors on i-motif and G4 formation has been studied in detail for the G4 forming sequence in the $c M Y C$ promoter $\left(\mathrm{NHE}-\mathrm{III}_{1}, 2.4 .2\right),{ }^{240}$ and the general requirements for a simultaneous formation and the possibility for a co-existence have been reviewed recently. ${ }^{235}$ Unsurprisingly, the population of either of the structures is dependent on ion-concentrations and $\mathrm{pH}$, but interestingly also on factors like superhelicity and molecular crowding. ${ }^{234,239,241}$ Most important to note is that the equilibrium between G4, i-motif and duplex DNA is highly competitive and dynamic. Under in vivo conditions, the effects of e.g. negative superhelical-stress (2.4.3), protein binding (2.3.2) and oxidative stress (2.3.3) greatly affect the interplay of non-canonical structures in the complementary G-/C-rich strands. 


\title{
2.3 Function and Targetability of DNA G-Quadruplexes
}

\author{
"If G-quadruplexes form so readily in vitro, \\ Nature will have found a way of using them in vivo"
}

Sir Aaron Klug, more than three decades ago ${ }^{242}$

\subsubsection{Occurence of G-Quadruplex Forming Sequences in the Human Genome}

G-rich sequences that are able to form G-quadruplexes are spread throughout the entire human genome, ${ }^{243-247}$ particularly in certain regulatory regions or functional domains, especially gene promoters of proto-oncogenes, ${ }^{243,248,249}$ immunoglobulin switch regions, ${ }^{250,251}$ telomeric ${ }^{210,228,252}$ and subtelomeric tandem repeats (minisatellites) ${ }^{91,253}$ and $5^{\prime}$-untranslated regions (5'-UTR) - $^{98,254}$. Furthermore, their formation is linked to $\mathrm{CpG}$ islands ${ }^{255}$ and chromatin modifications in the human genome. ${ }^{256-259}$ The consensus definition for G4 motifs:

$$
\mathbf{G}_{3-5} \mathbf{N}_{1-7} \mathbf{G}_{3-5} \mathbf{N}_{1-7} \mathbf{G}_{3-5} \mathbf{N}_{1-7} \mathbf{G}_{3-5}
$$

yields $\sim 376.000$ potential G4 motifs in the human genome. However, every bioinformatic algorithms has a trade-off for prediction to balance between false-positives and false-negatives. The above consensus definition does not account for non-canonical features of G4 polymorphism (2.1.3). A refined, sophisticated prediction tool called G4hunter ${ }^{260,261}$ found $\sim 700.000$ G4 motifs, which is in line with 716.310 distinct G4 structures that have been identified with a high-throughput sequencing method that is able to detect G4s in human genomic DNA. ${ }^{262}$ Out of this, 451.646 G4s have not been predicted by the consensus definition, ${ }^{249,263}$ which emphasizes both the overall significance of G4s in the human genome as well as the outstanding importance on non-canonical G4 structures.

Following this enlarged G4 definition, human subtelomeric tandem repeats (minisatellites) and their variations in Saccharomyces cerevisiae have come into focus for G4 function. ${ }^{91,96,253,264-267}$ The single stranded overhang in human telomeres consists of tandem repeats of the sequence:

\section{$($ TTAGGG)}

The formation, dynamics, structure and function of G4s in human telomeres, their impact on genome stability and their interaction with telomerase have been extensively studied ${ }^{252,268-273}$ and strategies to target telomeric-G4-structures ${ }^{205-210}$ have been discussed controversially in this context. ${ }^{274-289}$ The interested reader will find an astonishing plethora of papers and discussions in the literature, of which even a short summary would go beyond the scope of this discussion. For an updated overview, these most recent reviews on this topic are recommended. ${ }^{290-293}$ 


\subsection{Function and Targetability of DNA G-Quadruplexes}

Besides bioinformatic prediction, G4 structures in the human genome have been mapped and detected with various approaches ${ }^{246}$ using high-resolution sequencing (G4-seq) ${ }^{262}$, chromatin immunoprecipitation coupled to G4-specific single-chain antibody detection and high-throughput sequencing (G4-ChIP-seq ${ }^{294}$, qG4-ChIP-seq ${ }^{295}$ ).

The first in vivo detection (nuclear staining) of telomeric G4 structures has been reported in ciliates (Stylonychia lemnae) with single chain variable fragments of an in vitro selected antibody. ${ }^{246,296,297}$ Detection in fixed human cells has been reported with G4-specific antibodies BG4 $4^{66}, 1 \mathrm{H} 6^{298}$ and D $1^{299}$ using immunofluorescence microscopy. ${ }^{246}$

In-cell NMR has been used to investigate G-quadruplexes in living cells (in particular Xenopus laevis oocytes). ${ }^{300-306}$ Degradation of G4 forming oligonucleotides by endogenous nucleases is retarded, which elongates the obtainable time frame for NMR experiments. In-cell conditions allow the evaluation of the structural integrity (or structural changes) of G4 conformations under physiological relevant conditions with molecular crowding. The problems arising from broad signal linewidths and poor spectral resolution that are typically encountered for in-cell NMR spectra, can be evaded by using e.g. ${ }^{19} \mathrm{~F} \mathrm{NMR}^{307-309}$ ( or $\left.{ }^{31} \mathrm{P}\right)^{304,310}$ on chemically modified oligonucleotides.

More recently, small-molecule optical probes ${ }^{311}$ have been used to visualize G4 structures in live cells (IMT, ${ }^{67} \mathrm{BMVC}^{312}$ template-assembled synthetic G-quartets: N-TASQ ${ }^{313}$ ) even at the single molecule level. ${ }^{68}$ A fluorescent probe (DAOTA-M2) was used in combination with Fluorescence Lifetime

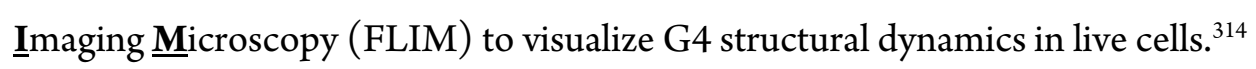

The advancements and efforts in the past decade ${ }^{246}$ towards mapping, detection, imaging and visualization of G4s in cellulo and in live cells now gives an astonishingly convincing view about the importance of G4 formation in the human genome. Today, there is no doubt anymore about the existence of G4s in human chromosomal DNA. Approaches like qG4-ChIP-seq (using G4-specific antibodies) and FLIM (using G4-specific fluorescent probes) now enable the investigation of G4 occurrence in relation to e.g. epigenetics, pathogenesis and carcinogenesis. ${ }^{259,315}$

Besides the potential role of DNA G-quadruplexes in the telomeric repeats, their function is discussed in basically all contexts that are related to genomic DNA. $247,251,259,315$ This includes in particular replication ${ }^{316-319}$, epigenetic processes such as $\mathrm{CpG}$ methylation ${ }^{255,320-323}$ or alterations to the chromatin structure $^{257,258,294}$, genomic instability ${ }^{96,253}$ and transcription ${ }^{324,325}$. Within the following chapters, the focus will be on DNA G4s as transcription regulatory elements. The group of S. Balasubramanian has recently published a comprehensive review ${ }^{259}$ (mini review in Trends in Chemistry) ${ }^{315}$ that covers the functions of both DNA and RNA G-quadruplexes. ${ }^{259,315}$ 


\subsubsection{Transcriptional Regulation of G-Quadruplexes and Binding-Interactions}

In a common, yet oversimplified picture of the regulatory function of G4s in gene promoters, transcription suppression (transcriptional off-switch) is proposed due to sterical hindrance and stalling of the RNA polymerase II (RNAP-II). However, even if further protein interactions are left unconsidered, the basic principles of how G4 can affect transcription efficiency are certainly more complex. In the context of transcription regulation, different modes of protein interactions have been discussed and similar effects are expected for small-molecules interactions. The concept of transcription regulation (on/off-switch) via small-molecule ligand targeting to G-quadruplexes did drive the field in the recent decades (see chapters 2.3.4 and 2.4.4). Unfortunately, regulation of genomic G-quadruplexes is not as straightforward as chemists like to imagine it. One aspect of a simple "actio-reactio" picture is, however, very well true: DNA G-quadruplexes can be represented as the starting point of the entire downstream cascades in cellular regulation - with all consequences to the complex cellular circuits. This chapter will therefore focus on the basic principles of G4 recognition. Two general types of interactions with G-quadruplex motifs will be differentiated: stabilizing/folding inducing and destabilizing/unfolding facilitating. Figure 13 shows an overview of the different modes of G4-regulated, altered transcription. The role of the complementary strand that can potentially form an i-motif is not discussed herein. To avoid a suggestive depiction, the strand is completely ignored in the Figure. Chapters 2.1.1 and 2.2.5 give implications for this issue. Here, it is important to note that the distribution of G4 forming sequences downstream of the transcription start site (TSS) is highly asymmetric in the sense and anti-sense strand (coding and non-coding strand). ${ }^{326}$ The location on either of the strands plays a crucial role for RNAP-II blocking, and can not only suppress but also activate transcription. The same holds true for the processing of G4s via helicases.

\section{G4-binding proteins}

Protein binding to G4 forming sequences has ambiguous effects on transcription levels: Some proteins have been reported to stabilize the G4 fold, while others inhibit G4 formation by binding to the single stranded DNA. G4-binding proteins can either inhibit the interaction with other proteins or recruit further proteins to form complexes. A prime example of a G-quadruplex binding and stabilizing protein is the nucleolar phosphoprotein nucleolin. ${ }^{327-333}$ Nucleolin was first found to bind G-G paired DNA ${ }^{334}$ and then reported to bind the $c M Y C$ promoter $\mathrm{G}^{335}$ and later also other promoter G4s (LTR promoter ${ }^{336,337}$ ). Binding is mediated via the C-terminus that contains RGG-repeats. ${ }^{338}$ There is growing evidence that nucleolin can differentiate between different conformations and preferentially binds long-looped G4 conformations. ${ }^{339,340}$ This has strong implications for the recognition of different folded isomers (e.g. spare-tire isomers) as has been outlined for the $c M Y C$ promoter G4 (see chapters

2.4.2 and 2.4.3). ${ }^{341,342}$ Recently, the interactions with RNA G4s have been used to target nucleolin. ${ }^{343-345}$ 

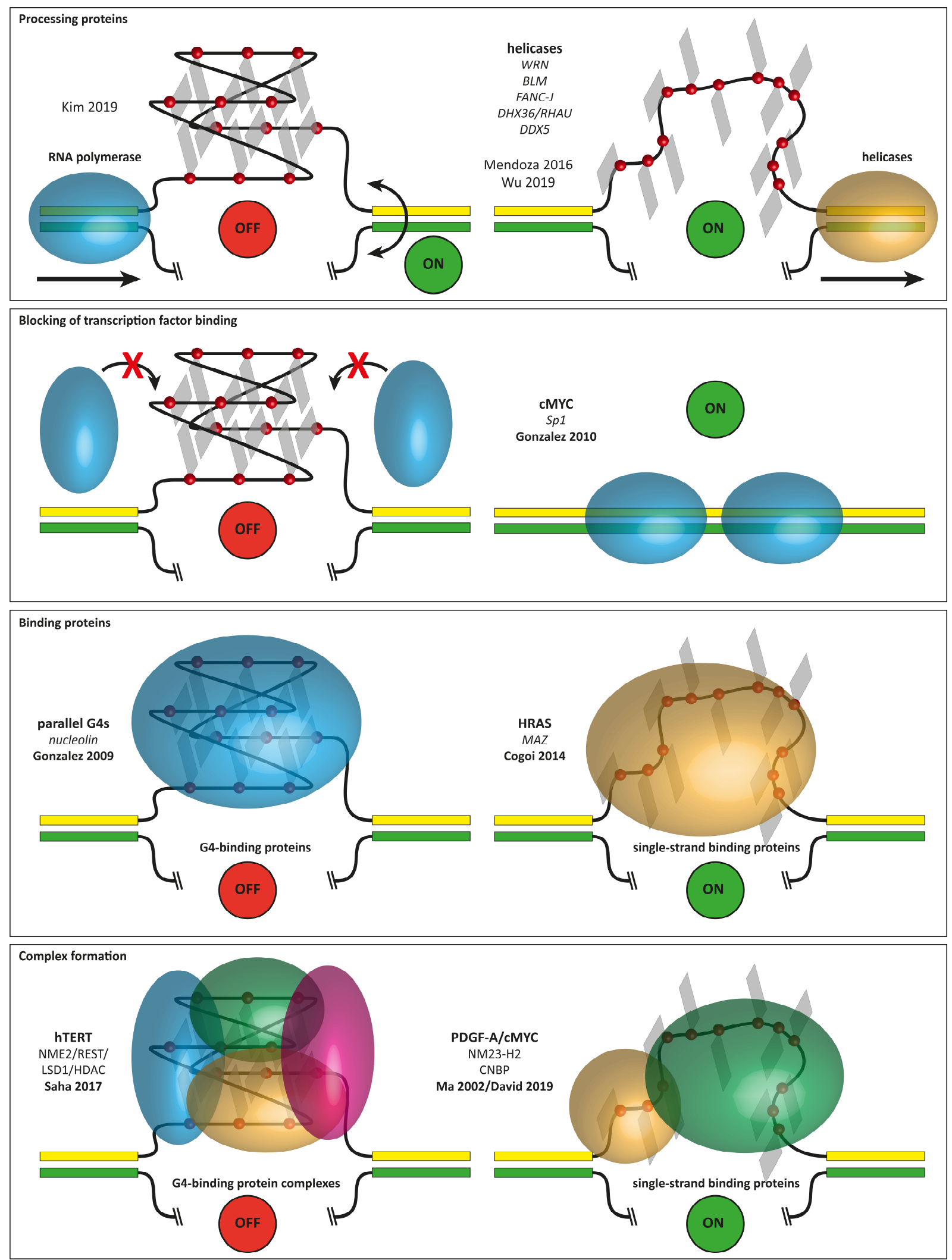

Figure 13: Different mechanisms of transcription regulation with G4-interacting proteins. Processing proteins: Classical model that shows stalling of RNA polymerase (RNAPII) during transcription due to sterical reasons (inverse effects for G4s in the coding/non-coding strand). ${ }^{324}$ The G4-fold cannot be resolved by the RNAPII itself, but helicases ${ }^{346}$ like WRN, BLM, FANC-J, RHAU or DDX $5^{347}$ are effective G4 unwinders. Binding proteins: Proteins that bind and stabilize G4s can downregulate the transcription, while proteins that bind the unfolded single strand (and/or facilitate the unfolding) can have an activating effect. Complex formation: Some G4 binding proteins recruit additional proteins to form complexes, which can act by either silencing or activating. 
A manifold of G4-binding proteins have been reported (e.g. Zuo1 ${ }^{348}$, IFI16 $6^{349}$, Lia3 ${ }^{350}$, LARK ${ }^{351}$, SLIRP $^{352}, \mathrm{CNBP}^{353,354}$ ) and reviewed recently. ${ }^{355,356}$ The analysis of shared motifs ${ }^{357}$ and the general interaction with RGG-domains ${ }^{358-360}$ have been analyzed and a powerful tool to explore G4-interacting proteins called G4IPDB (a web-based database with more than 200 entries) is now available. ${ }^{361}$
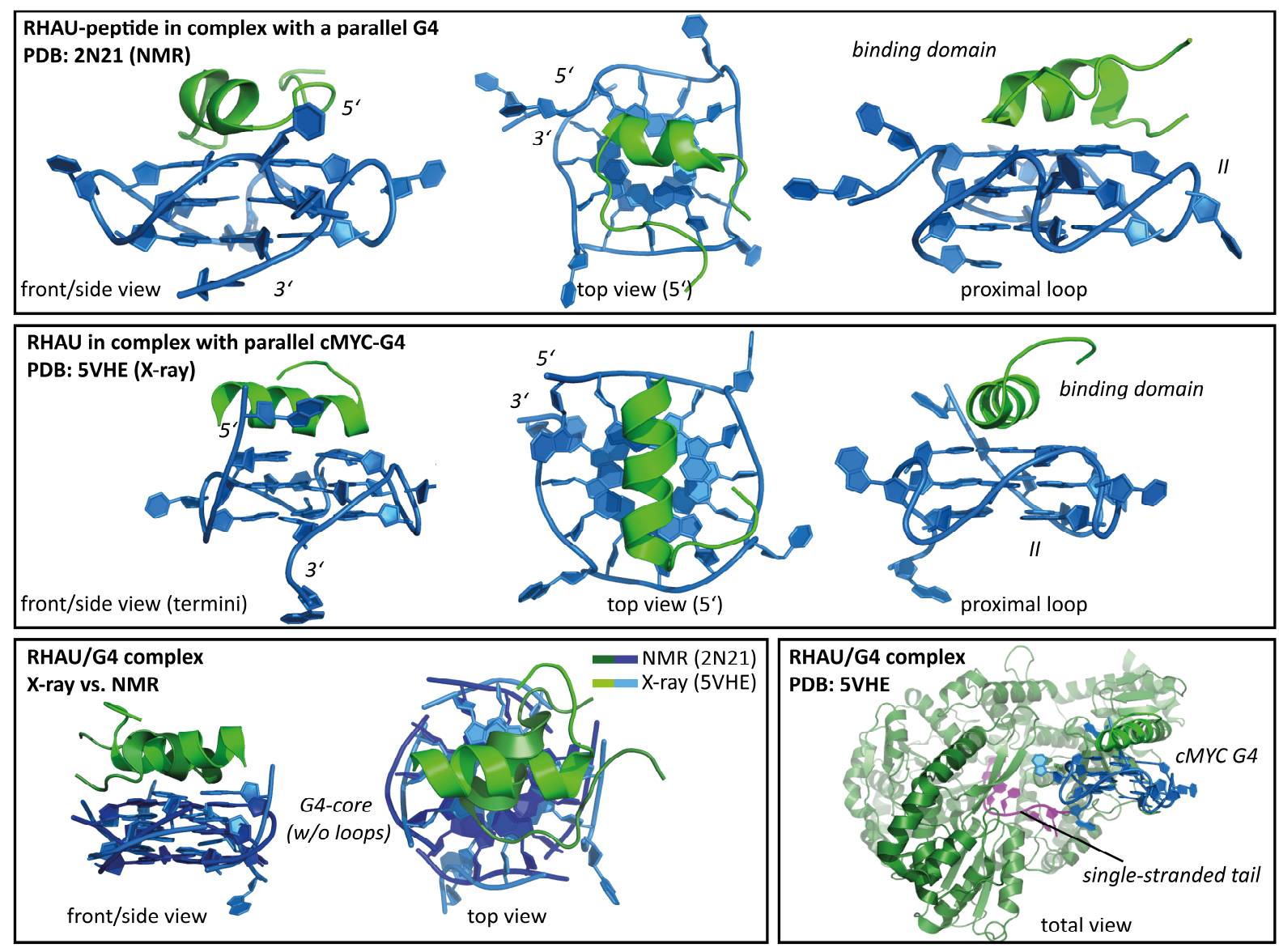

Figure 14: Details of the PDB-structures of the RHAU-peptide (binding motif) from NMR spectroscopy (PDB: 2N21) ) $^{362}$ and the whole RHAU helicase from X-ray crystallography (PDB: 5VHE, detail of binding domain, entire protein complex at the bottom-right $)^{363}$. The comparison of the structures shows different orientation of the binding domain at the toptetrad (nearly perpendicular arrangement).

\section{G4-unwinding proteins and helicases}

Some interacting proteins have been shown to facilitate G4 unfolding or directly bind to the unfolded single-strand and thereby disturb G4 formation (MAZ ${ }^{364,365}, \mathrm{NM} 32-\mathrm{H} 2^{366-368}$ ). Recent studies suggest that CNBP-binding acts in a comparable manner. ${ }^{354}$ Information on the detailed binding modes of these proteins remains ambiguous ${ }^{369,370}$ and NM32-H2 has also been discussed in a different context as mediator for epigenetic suppression of the $h T E R T$ promoter (recruiting a REST-LSD1 complex). ${ }^{371}$ Different helicases have been reported (DEAH-helicases: DHX36/RHAU ${ }^{362,363}$, SF2 helicase: FANC-J ${ }^{298,372-374}$, SF1b helicase (S. cerevisiae): Pif1 $253,264-266,375,376$, RecQ-helicases ${ }^{147,377-379}$ : BLM ${ }^{380-384}$, $\left.\mathrm{WRN}^{385,386}, \operatorname{RecQ} 5^{387}, \operatorname{RecQL4}{ }^{388-390}\right)$ that are able to process and unwind G-quadruplexes. ${ }^{346,391} \mathrm{G} 4$ unwinding ability is vastly different for the members of the RecQ helicase family, with BLM and WRN showing the highest efficiency. For some helicases a topology-specific unwinding activity has been 


\subsection{Function and Targetability of DNA G-Quadruplexes}

shown. ${ }^{380,392}$ Interestingly, some are strictly unable to unwind G4s (RecQ1) ${ }^{393}$, which has implications for the recognition of distinct templates. Helicase-deficiencies in general are linked to severe hereditary diseases such as Bloom-syndrome (age-unrelated cancer predisposition, BLM-deficient) ${ }^{394}$, Wernersyndrome (pre-mature aging phenomena, WRN-deficient) ${ }^{395}$ or Rothmund-Thomson syndrome (accelerated aging, RecQL4-deficient) ${ }^{396}$. The main cause of this is a chromosome instability that is also associated to the inability to resolve G-quadruplexes in the genome. How the helicases recognize specifically the G4-fold still remains largely elusive. Figure 14 shows the binding of RHAU helicase to different parallel G4 conformations. Interestingly, the peptide binding domain of RHAU is arranged differently in a NMR-structure (PDB: $2 \mathrm{~N} 21$, peptide only) ${ }^{362}$ compared to the X-ray-structure (PDB: 5VHE, whole protein) ${ }^{363}$. More recently, the DEAD-box helicase DDX5 has been linked directly to transcriptional activation of $c M Y C .{ }^{347}$ DDX5 is overexpressed in cancer cells and might have an important function as G4-resolvase that contributes to the high expression levels of MYC. Sterical hindrance of unprocessed G4s is further important for the binding of transcription factors that bind to B-form, double-stranded DNA (e.g. Sp1 ${ }^{397,398}$ ). The formation of G4s thus blocks the binding sites of transcription factors and thereby act as transcriptional silencer. ${ }^{369}$

\section{$\underline{\text { Small-molecule binding }}$}

Around 2000, the first studies were published that report on specific promoter G4-ligand binding (cationic porphyrins). ${ }^{399-401}$ Since then, a wide range of ligand scaffolds has been proposed, synthesized and studied for specific G-quadruplex targeting. ${ }^{402,403}$ A discussion of small-molecule structural classes and strategies to bind specific G-quadruplexes is far beyond the scope of this thesis and the interested reader will find countless comprehensive reviews in the literature. (see some non-comprehensive, exemplary references: selected studies ${ }^{280,404-410}$, more recent studies ${ }^{410-413}$ and for further analysis of G4-ligands the web-based database G4LDB can be used ${ }^{415}$ ) Figure 15 shows different structures of ligand-G4 complexes taken from the PDB that all feature the most common binding mode for G4-ligands, which is stacking to the tetrads via large $\pi$-electron surfaces. The NMR-structure of an $\mathrm{Au}(\mathrm{III})$-ligand in complex with a telomeric hybrid-2 G4 (PDB: 5MVB) ${ }^{416}$ shows an interesting rearrangement of the capping structures that are required to accommodate the ligand. An important point to mention here is that all of these studies aim to stabilize the G4-fold and modulate its function by shifting the thermodynamic equilibrium towards a folded state. The group of D. Monchaud recently conceptualized an alternative, orthogonal approach that aims to destabilize certain G4 conformations. ${ }^{417}$ The different strategies that have been proposed to target G4s with small molecules show the diversity of possible effects that result from interfering with G4-regulation. 

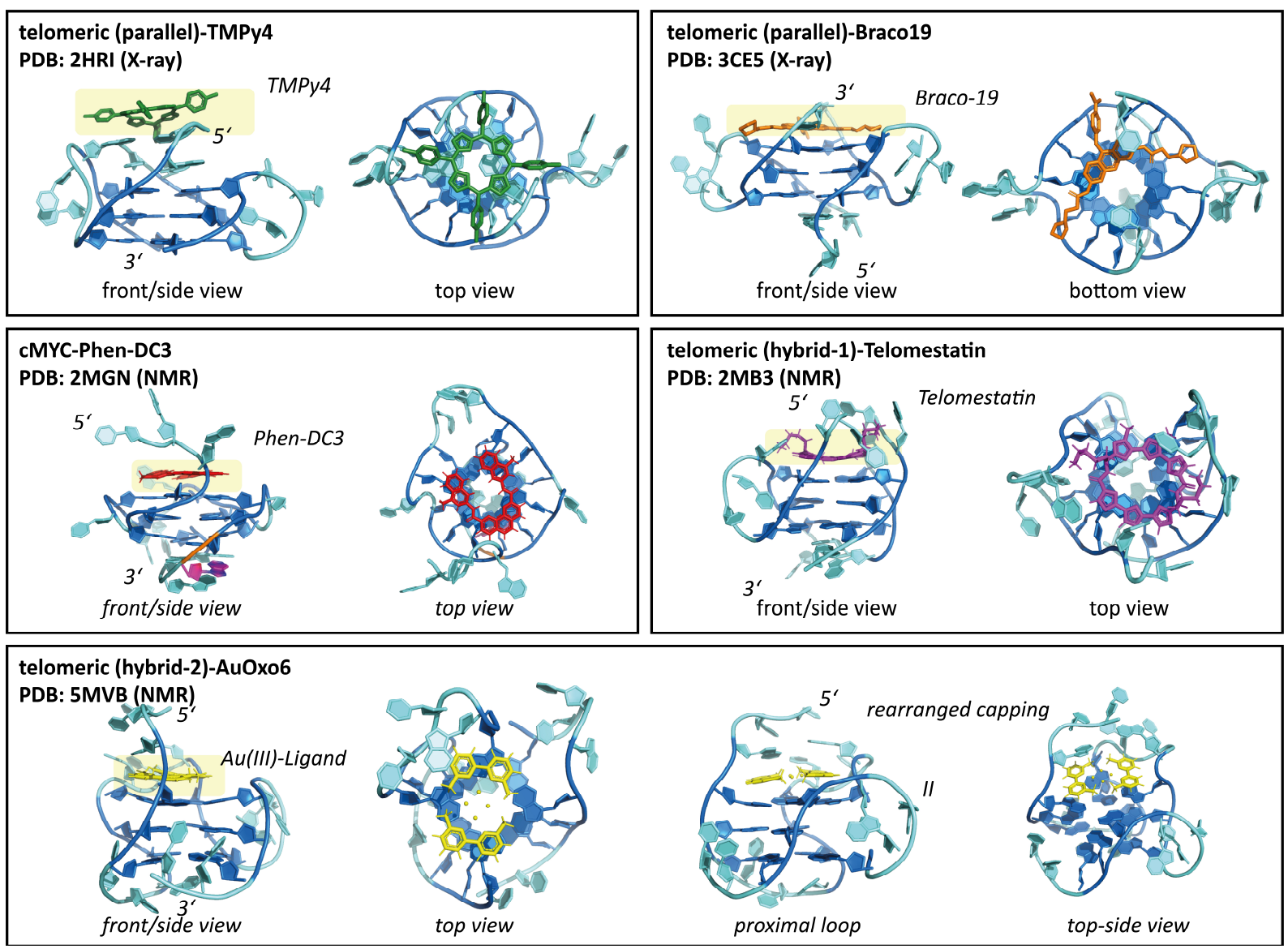

Figure 15: Details of the PDB-structures of G-quadruplex-ligand complexes that feature $\pi$-stacking to the top $\left(5^{\prime}\right)$ or bottom (3') tetrads as a common binding mode. The complexes show a dimeric, parallel telomeric G4 with TMPy $4^{401,418,419}$ (PDB: 2HRI) ${ }^{420}$ and Braco 19286,421 (PDB: 3CE5) ${ }^{422}$; a cMYC G4 with Phen-DC3 ${ }^{423,424}$ (PDB: $2 \mathrm{MGN}{ }^{425}$; a telomeric hybrid-1 G4 with Telomestatin ${ }^{409}$ (PDB: $\left.2 \mathrm{MB} 3\right)^{426}$ and a telomeric hybrid-2 G4 with an Au(III)-ligand (PDB: 5MVB, AuOxo6) $)^{416,427-429}$.

\subsubsection{Oxidative Damage under Cellular Stress Conditions}

Guanine is the most electron rich from all four DNA nucleobases and therefore the most prone to oxidation. In G-rich runs this effect is even increased, since due to $\pi$-stacking of Gs the ionization potential of the 5'-G is significantly lowered with increasing length of the G-tract. Duplex DNA has the ability to funnel electron holes (oxidation sites) through the $\pi$-stacked nucleobases. These electron holes can migrate through the DNA duplex until they reach the site that is most prone to oxidation, which will likely be a 5'-G of G-runs. This has been hypothesized to serve as cathodic protection of genes ${ }^{430}$ or epigenetic mark to modulate gene expression. ${ }^{431}$ The consequences of oxidative damage to G-residues and G-quadruplex formation in the genome have been conceptualized and reported in a series of publications from C. Burrows in the last years ${ }^{113,254,431-439}$ and have been recently reviewed. ${ }^{440}$ 8-oxo-guanine $(\mathrm{OG})$ is the major oxidation product that results from a two-electron oxidation (Figure 16) with reactive oxygen species (ROS). OG can be further oxidized (four-electron hyperoxidation) by ROS to 5-guanidinohydantoin (Gh) or spiroiminodihydantoin (Sp). Oxidized G-residues with lesions can be repaired by OG-glycosylase (OGG1) or NEIL-glycosylases ${ }^{441}$ that 
initiate the base excision repair (BER). Those oxidized species alter the Hoogsteen interface of G-residues and disturb the tetrad formation in G-quadruplexes. ${ }^{442-444}$ The $\mathrm{N}-\mathrm{H} 7$ imino proton enables the formation of A-G Hoogsteen base pairing and the O8 causes a slight sterical clash, if base paired with $\mathrm{C}$. These effects cause a mutagenic potential (during replication) towards a G-to-T transversion, when the OGs are not repaired.

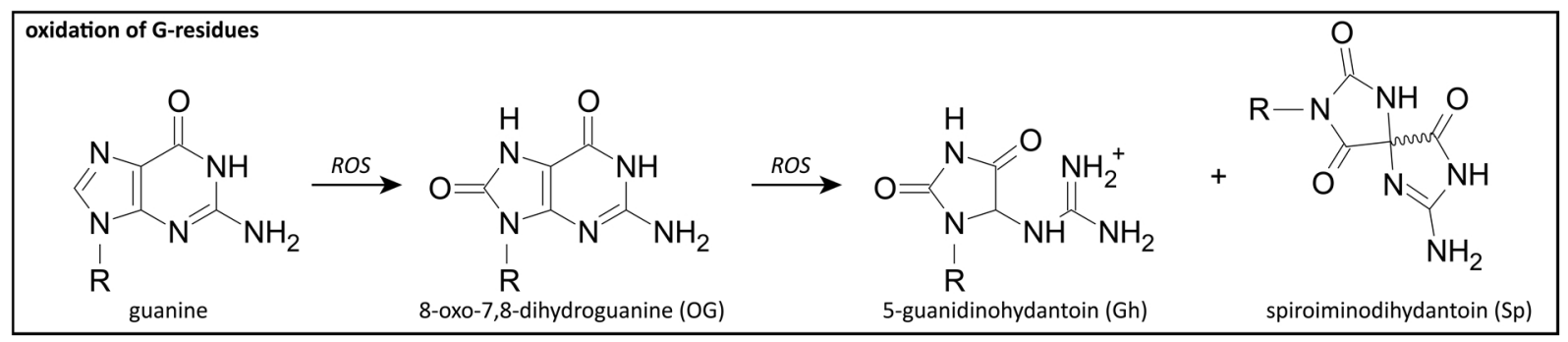

Figure 16: Major oxidation products for the reaction of guanine with reactive oxygen species (ROS). 8-oxo-7,8-dihydroguanine (OG) as oxidation product of a two-electron oxidation; 5-guanidinohydantoin (Gh) and spiroiminodihydantoin $(\mathrm{Sp})$ are the hyperoxidation products of a four-electron oxidation.

Many G-quadruplex forming regions in oncogene promoters possess more than four G-rich tracts that are close by in sequence. ${ }^{113}$ The consequences of this for a possible non-canonical structural polymorphism have been discussed in chapter 2.1.2. Burrows and co-workers have outlined a possible evolved function for these spare-tire G-tracts in response to oxidative damage in one of the G-rich tracks. ${ }^{113,438}$ They propose a mechanism that describes a maintaining of function for G4s. The G-tract that carries a lesion is excluded from G4 formation and one of the spare-tires participates instead in G4 folding as surrogate. The so exposed oxidized residues are accessible to the BER machinery. This mechanism has also been utilized for an approach with an intermolecular spare-tire surrogate (using a PNA) to recover the G4 functionality. ${ }^{445}$ Further, OG sites in folded G4s (shown for the polymorphic KRAS G4 ${ }^{446-448}$ ) have been shown to be recognition sites for the $M Y C$ associated zinc finger protein (MAZ). ${ }^{364,365}$ MAZ binds to oxidized G-residues in the loops of folded G4s and facilitates unfolding and subsequent activation for BER. This finding has implications for the recognition of oxidized Gs in the loops, that can emerge from G-register isomers or G-quadruplexes with G-vacancy sites (2.1.3).

\subsubsection{Strategies in Anti-Cancer Treatment}

The stabilizing effect of small-molecule binding has now developed to a strategy that aims to target specifically G-quadruplexes in the human genome for anti-cancer treatment. ${ }^{276,282,449-451}$ The first reports on G-quadruplex targeting mainly aimed for telomeres and telomerase inhibition. ${ }^{274}$ This strategy is discussed controversially and the biological implications for it have shifted, since the functionality and structural assembly of human telomeres still remains largely elusive. One of the main ideas was to block telomerase interactions by facilitating G-quadruplex formation in the telomeres. This prevents the limitless replicative potential of cancer cells and re-enables senescence and 
apoptosis. However, the alteration of telomere capping structures and shelterin complexes overrule this effect and lead to genomic instability and the activation of DNA repair machineries. ${ }^{284-288,449}$

One of the very first studies on G-quadruplex formation in the $c M Y C$ promoter showed that expression levels can be significantly reduced after binding of a small molecule. ${ }^{452}$ This strategy has now evolved and G-quadruplexes in gene promoters are recognized as cis-acting elements that can be directly targeted to regulate transcription levels. ${ }^{54,90}$

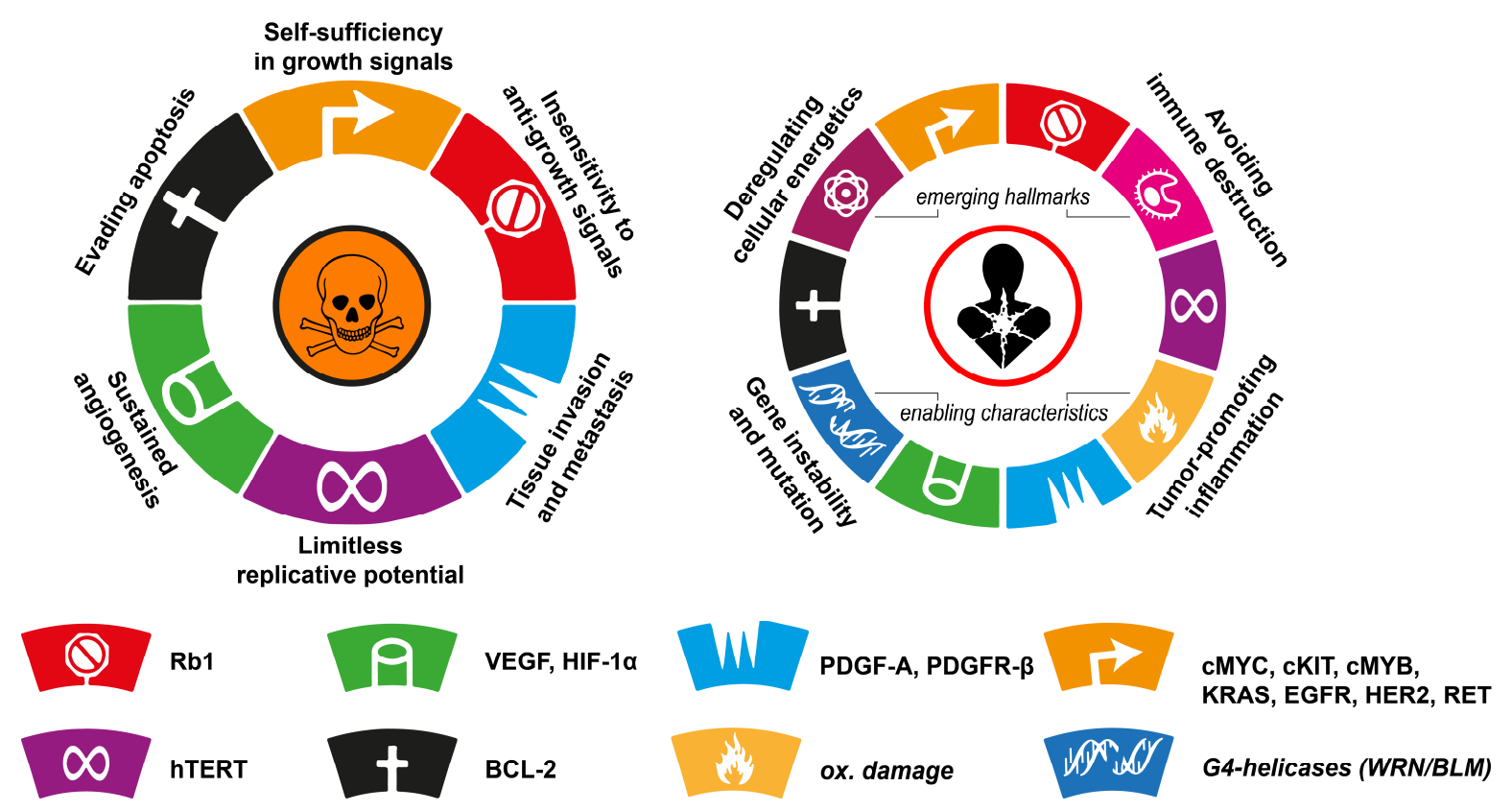

Figure 17: The six "classical" hallmarks of cancer according to Hanahan and Weinberg 2000 (left). ${ }^{453}$ Refined and enlarged definition ("next generation", 2011), including enabling characteristics and emerging hallmarks (right). ${ }^{44,455}$ (bottom) Overview of genes with reported regulation through promoter G-quadruplexes or mechanisms that affect G4 regulation (italic). ${ }^{239,456-459}$ Schematic depiction adapted from Hanahan and Weinberg. ${ }^{453,454}$

An analysis of the gene ontology of promoters with G4 forming sequences reveals that the enrichment of promoter G4s is unequally distributed among different gene functions. ${ }^{244,249}$ Gene ontology categories with significantly higher frequencies of $\mathrm{G} 4$ forming sequences are linked to e.g. transcription factor activity, RNAPII transcription factor activity, neurogenesis, kinase activity and cell differentiation, while categories like immune response, nucleic acid binding, protein biosynthesis, ribosome-related or antigen binding have significantly lower frequencies of G4 forming sequences. This aspect of genomic G4-distribution highlights the general relevance for G4 regulation in cancer cells and their importance as molecular targets. ${ }^{459}$ Structural validation of G-quadruplex forming sequences has been reported for the promoters of many important genes that can be assigned to all different hallmarks of cancer (Figure 17). ${ }^{239,244,450,453}$ Among them are (non-comprehensive): cMYC $C^{99,342,460,461}, B C L-2^{126,217,462-464}, h T E R T^{102,103,115,116}, V E G F^{85,408}, c M Y B^{90}, c K I T^{130,465}, K_{R A S}^{365,446,447,466}$,

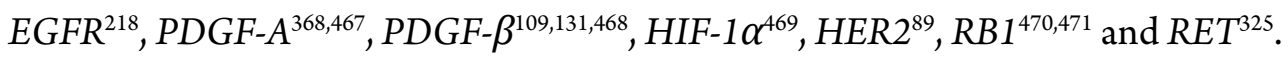




\subsection{The cMYC Proto-Oncogene Promoter}

"I am, of course, most ignorant about all things biological, but I imagine most (X-ray) people start that way."

Rosalind Franklin, response to John Randall $1951^{472}$

\subsubsection{The cellular Myelocytomatosis Protooncogene}

Information within this chapter on molecular structure, biological function, oncogenic potential and targeting of MYC have been extensively reviewed in the last years. ${ }^{473-479}$ MYC is a family of protooncogenes that was found after the discovery of oncogenic retroviruses that cause myelocytomatosis ( $v$-myc oncogene). The MYC-family has three members, namely $c M Y C$ (or just $M Y C), N-M Y C$ and $L-M Y C$ that encode for transcription factors. The C-terminal domains of MYC-members have basic-helix-loop-helix (bHLH) and leucine zipper motifs. This C-terminal domain remains largely unstructured until dimerization with other transcription factors such as the myc-associated factor $\mathrm{X}(M A X)$. The bHLH enables the binding to DNA, while the leucine zipper allows dimerization. Figure 18 shows the X-ray structure of a MYC-MAX heterodimer bound to DNA (PDB: 1NKP). ${ }^{480}$ The unstructured $\mathrm{N}$-terminal domain is responsible for transcription regulation (transactivation). It contains highly conserved myc-boxes and forms complexes with other factors that induce structured folding upon binding.

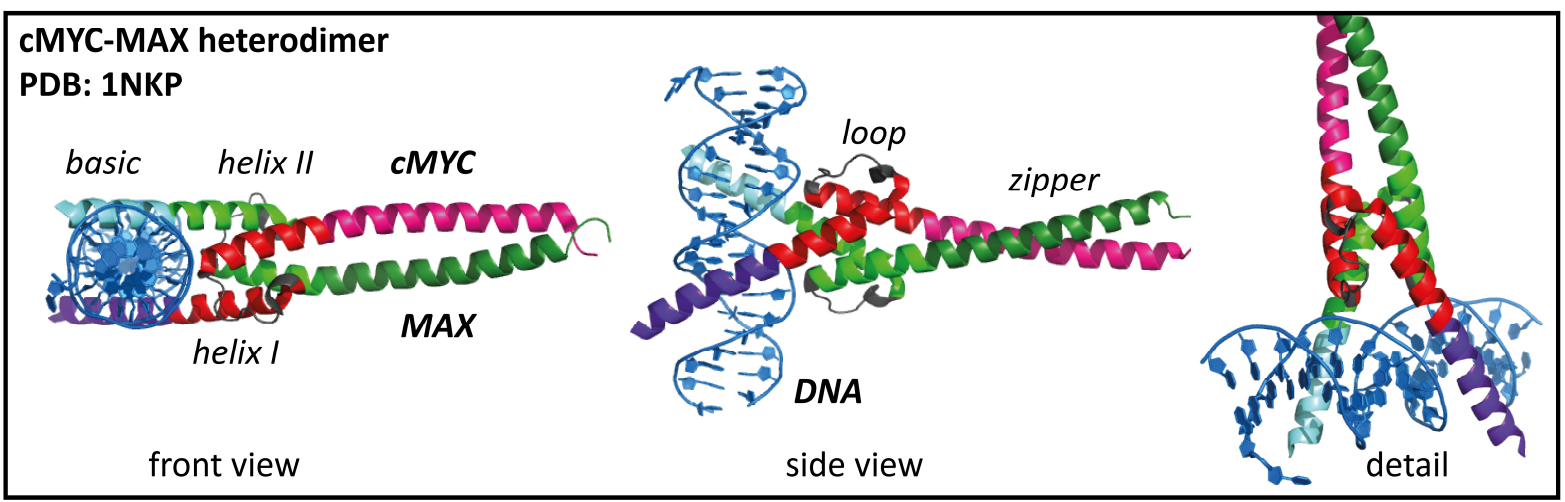

Figure 18: Crystal structure of a $c M Y C-M A X$ heterodimer bound to DNA (PDB: 1NKP). $c M Y C$ is shown in red/violet colours and MAX in green/cyan colours. The structure of the C-terminal domain comprises a basic-helix-loop-helix (bHLH) and a leucine zipper motif.

$M Y C$, as a super or master transcription factor plays many important roles and regulates more than $15 \%$ of the human genome. A major function of $M Y C$ is cell proliferation, growth and differentiation as wells as apoptosis. In this function, it has a high transformation potential and is a key driver in tumorigenesis. However, strategies for small-molecule drug development to target $M Y C$ are extremely challenging, mainly because it lacks a specific active binding site. Hence, $M Y C$ is considered undruggable. ${ }^{481}$ 
Usually, the expression of $M Y C$ is highly regulated through the tight control of its promoter region. However, MYC is found deregulated in a wide range of nearly $70 \%$ of human cancers with elevated levels of MYC-expression. Burkitt's lymphoma, a highly aggressive B-cell non-Hodgkin lymphoma, is a prime example for the devastating effect of $c M Y C$ misregulation. Here, chromosomal translocation to an ectopic promoter leads to an activation of the $c M Y C$ oncogene, thereby making it one of the fastest growing tumour in humans. ${ }^{482}$

A plethora of molecular mechanisms maintains cellular homeostasis of MYC, many of them are still poorly understood. ${ }^{473,483,484}$ What is known is that the promoter is controlled and regulated by a large number of cis-regulatory elements, intra- and extracellular signals and signal pathways associated to an array of transcription factors that directly bind to the $c M Y C$ promoters:

"Everything regulates MYC. From stem cells and proliferation to senescence and cell death, MYC participates in almost every crucial decision of almost every cell. Hundreds of extracellular and intracellular signals, operating through an array of transcription factors, chromatin modifiers/remodelers, and regulatory RNAs (recruited to or synthesized at the c-myc locus), are brought to cis-elements vicinal to the promoter or strewn across a still poorly delineated chromosomal domain and are all somehow integrated to set the physiological levels of $c-m y c m R N A . "$

David Levens, "You Don't Muck with MYC" (2010) 484

Hence, unravelling the regulation, function and role of MYC has remained an "enduring enigma"

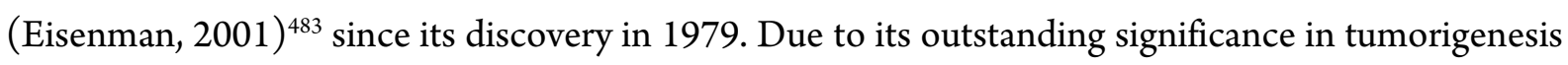
and the impracticality of a direct $M Y C$ targeting, a focus on the gene promoter region has evolved. Soon after sequencing the $c M Y C$ locus $^{485}$ a nuclease $S 1$ analysis revealed several altered DNA secondary structures in slow equilibrium with a canonical B-form helix in the promoter region. ${ }^{486}$ DNase 1 mapping of this region then localized several nuclease hypersensitive sites. ${ }^{487}$ At one of these sites, the nuclease hypersensitive element $\mathrm{III}_{1}\left(\mathrm{NHE}-\mathrm{III}_{1}\right)$ located -142 to -115 bp upstream of the $\mathrm{P}_{1}$ promoter a high strand asymmetry was found with a purine ( $\mathrm{Pu}, \mathrm{G}$-rich) and a pyrimidine (Py, C-rich) strand. In the late ' $80 \mathrm{~s}$ it was hypothesized that a regulation via triplex formation might be a plausible mechanism that could explain the vulnerability against nucleases at these sites. Two structures have been proposed that involve either a complex tandem $\mathrm{H}$-DNA with two intramolecular Py-Pu-Py triplexes ${ }^{488}$ or an intermolecular $\mathrm{Pu}-\mathrm{Py}$-Pu hybrid-triplex. ${ }^{54}$ Targeting this site with anti-sense RNAs indeed repressed transcription in vitro. ${ }^{489}$ The role of interactions with transcription factors was discussed for both models. ${ }^{40,491}$ However, $\mathrm{Py}-\mathrm{Pu}-\mathrm{Py}$ triplexes require low $\mathrm{pH}^{488,491}$ and $\mathrm{Pu}-\mathrm{Py}-\mathrm{Pu}$ triplexes require 5-20 $\mathrm{mM}$ magnesium ions ${ }^{489,492} .493$ Both requirements do not fit physiological conditions. 


\subsection{The cMYC Proto-Oncogene Promoter}

In the mid '90s it was found that the structural dynamics in the $c M Y C$ promoter region are highly iondependent and that especially physiological concentrations of $\mathrm{K}^{+}$inhibit a potential triplex formation and competitive triplex/quadruplex equilibria have been proposed. ${ }^{492}$ Finally, in 1998 the formation of a tetraplex; ${ }^{493}$ and in 2000 the formation of an i-tetraplex ${ }^{494}$ in the complementary strand of the cMYC NHE-III ${ }_{1}$ region has been proposed.

However, even after the discovery of the G-quadruplex formation in the $c M Y C$ promoter, a possible biological function of triplex (inter- and intramolecular) formation is still in discussion. ${ }^{495-497}$ The promoter can be targeted with triplex-forming oligonucleotides (TFOs) that have been shown to downregulate the transcription and this effect is particularly intensified in cancer cells. ${ }^{498-503}$ TFO-targeting strategies can benefit from an increased cellular uptake and improved intracellular stability of homologous G4 forming oligonucleotides (also shown for VEGF ${ }^{504}$ ). ${ }^{502}$ Strategies based on siRNAs and anti-sense oligonucleotides have also been developed to target not the genomic DNA, but inhibit the MYC mRNA. ${ }^{505}$ Further, the promoter region is a mutagenic hotspot; ${ }^{495,497}$ the mutations and single nucleotide polymorphism (SNP) have been mapped and linked to strong effects on transcription levels. ${ }^{506,507}$ This has severe implications for the delicate equilibrium of all possible noncanonical DNA conformations and these mechanisms have to be considered in the general context of the biological function and targetability of G-quadruplexes in oncogene promoters (chapters 2.3.4 and 2.4.4). In conclusion, this chapter cannot be summarized better than with this quote from a recent review:

"Thus, the c-myc promoter is still something of a black box and only one point is certain: regulation of the c-myc promoter is extremely complex with a lot of redundancy, many feedback loops, and several cross-regulatory circuits involved."

Inken Wierstra and Jürgen Alves, The $c-m y c$ Promoter: Still $\underline{M} y$ ster $\underline{Y}$ and $\underline{C}$ hallenge $(2008)^{473}$

\subsubsection{Nuclease Hypersensitive Element III $_{1}$}

The $c M Y C$ promoter region (Figure 19) has four promoters $\left(\mathrm{P}_{0}, \mathrm{P}_{1}, \mathrm{P}_{2}\right.$ and $\left.\mathrm{P}_{3}\right)$ and seven nuclease hypersensitive elements (NHE). $75-90 \%$ of transcription initiates at $\mathrm{P}_{2}, 10-25 \%$ at $\mathrm{P}_{1}$ and less than $10 \%$ at $\mathrm{P}_{0}$ and $\mathrm{P}_{3}$. The NHE- $\mathrm{III}_{1}$ spans a $27 \mathrm{bp}(\mathrm{Pu} 27)$ sequence -142 to $-115 \mathrm{bp}$ upstream of the $\mathrm{P}_{1}$ promoter. The NHE-III ${ }_{1}$ has been shown to control up to $90 \%$ of total transcriptional activation of cMYC. ${ }^{369,452,473}$ Transcriptional regulation through the $\mathrm{NHE}^{-\mathrm{III}_{1}}$ is believed to be primarily mediated through G-quadruplex formation ${ }^{99,452,493,508}$ in the G-rich strand (Pu27), but the role of i-motif formation in the complementary C-rich strand (Py27) has also been discussed. ${ }^{83,241,509-511}$ This chapter will focus on models on the G4-mediated transcription control (see also chapter 2.3.2 for general considerations). 


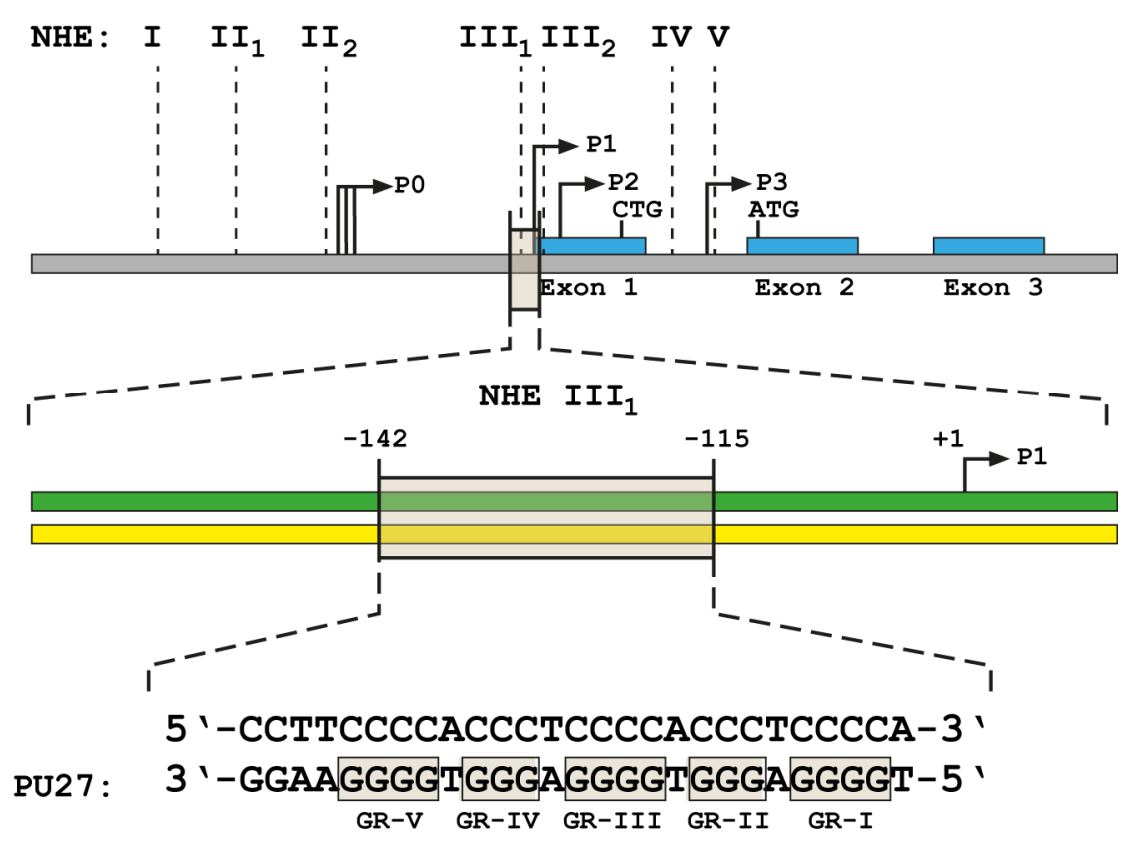

Figure 19: Depiction of the $c M Y C$ promoter region including nuclease hypersensitive elements (NHE) I-V, promoters 0-3 and transcriptional start sites. G-runs I-V in the NHE- $\mathrm{III}_{1}$ are numbered from 5'-to-3' direction Figure has been adapted according to the publications from the Hurley group. ${ }^{99,452}$

The group of L. Hurley has outlined a model for the transcriptional regulation of the NHE-III $I_{1}$ in the last two decades. ${ }^{369,456,512}$ Figure 20 shows a schematic representation of the regulatory model that

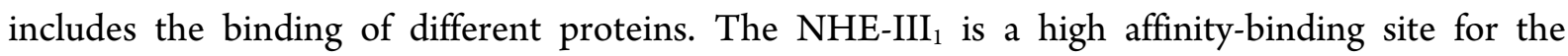
transcription factor Sp 1. ${ }^{369,397,398}$ In cells that express low levels of MYC the promoter is not occupied by $\mathrm{Sp} 1$, but serum stimulation induces $M Y C$ transcription and results in binding of Sp1. ${ }^{369,513}$ CNBP $^{353,354}$ unfolds the G4, binds the Pu27 single strand and has been shown to induce MYC expression. ${ }^{359,369,514}$ hnRNP K acts as transcription factor, binds the Py27 single strand and activates transcription. ${ }^{369,515,516}$ NM23-H2 has been shown to bind both the single-stranded Pu27 and Py27 and drive the unfolding of the G4 structure. ${ }^{366,367,369,517-519}$ Nucleolin recognizes and binds the G-quadruplex structure and stabilizes the folded conformation, which silences the MYC transcription (see also chapter 2.3.2). ${ }^{335,338,369}$

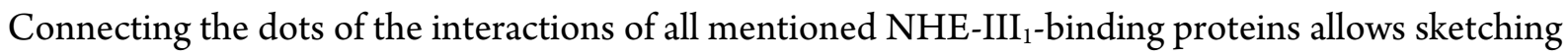
the regulatory model shown in Figure 20. In earlier studies, it was also proposed that hnRNP A1 binding to Pu27 is involved in $c M Y C-N H E-\mathrm{III}_{1}$ regulation, but due to its weak affinity in competitive assays is now considered not to take part in these processes. ${ }^{48,493,520}$ The role of hnRNP A1 is still discussed for transcriptional regulation in the KRAS promoter. ${ }^{448}$

In this model, a pre-requisite for G-quadruplex formation is a local unwinding with strand separation, in particular because of negative supercoiling. Negative supercoiling is typically induced during transcription, ${ }^{456,521}$ which implies that G4 formation is favoured after transcription is already initiated. 


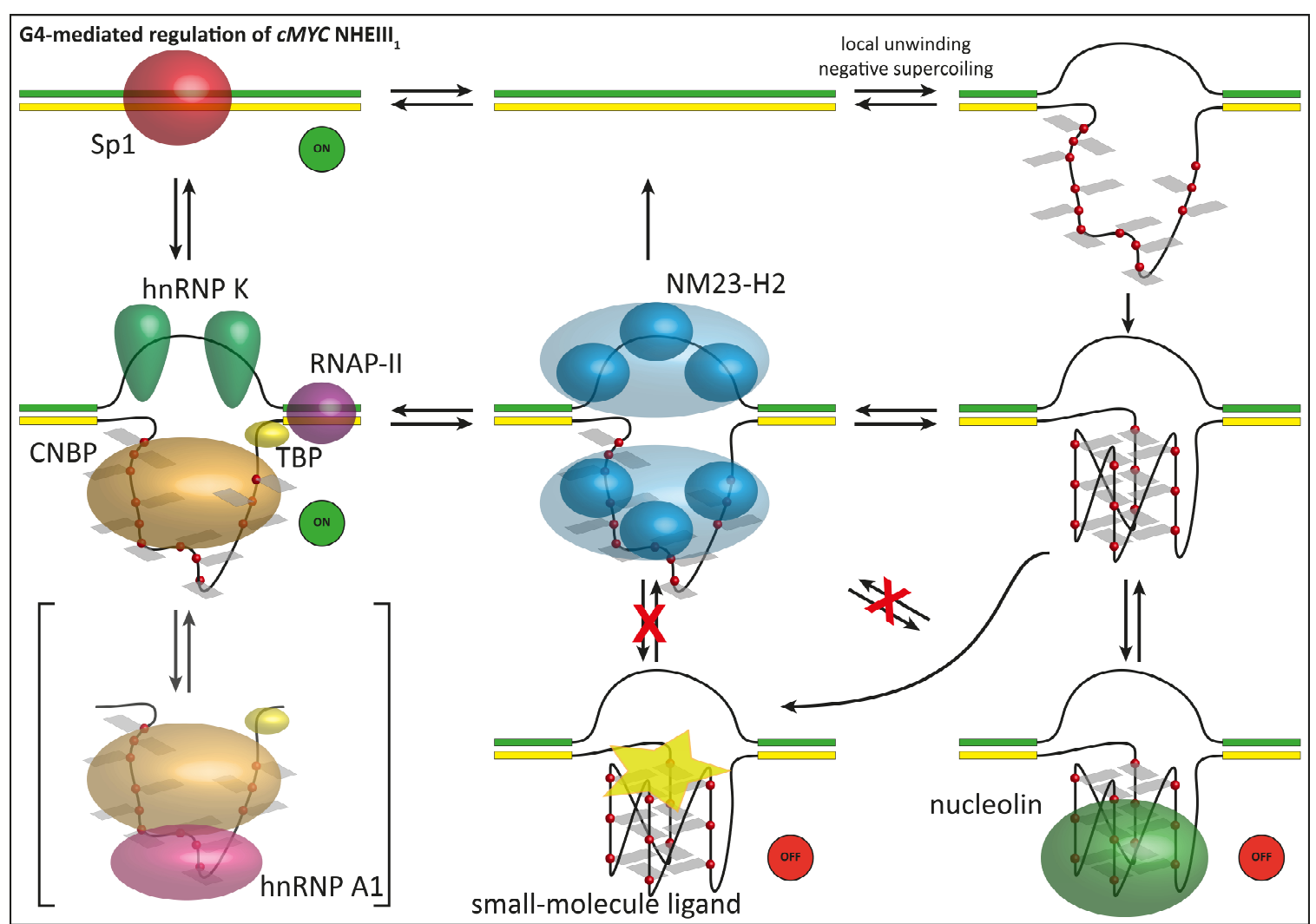

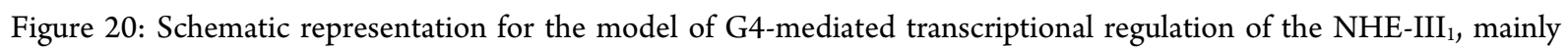
proposed by Hurley and co-workers. ${ }^{369}$ (left) Transcription factors like Sp1 bind the double stranded B-form DNA and initiate transcriptional activation. A complex of CNBP and TBP (TATA-box) binding protein and hnRNP K binding to the complementary strand enables recruitment of RNA Polymerase-II (RNAPII). Initial studies by Simonsson et al. proposed the involvement of hnRNP A1 binding, ${ }^{493}$ but the binding affinity of hnRNP Al is comparably weak. ${ }^{448,520}$ NM23-H2 facilitates the unfolding of the G4-fold and enables reformation of the double strand or recruitment of other proteins. (right) Negative superhelicity induces strand separation and G4 formation. The G-quadruplex can be processed by destabilizing proteins or can be bound by nucleolin, which then acts as transcriptional suppressor. Binding of smallmolecule ligands interferes with protein binding and stabilizes the G4 in a non-protein-bound state.

\subsubsection{Structures and Dynamics of the cMYC G-Quadruplex}

\subsubsection{High-Resolution Structures}

Within the first report on G-quadruplex formation in the $c M Y C$ promoter in $2002,{ }^{452,508}$ two anti-parallel structure models were proposed based on DMS-footprinting, namely chair or basket conformations. This model has been revised in 2004, based on simple CD-spectra and gelelectrophoresis to find that the 3'-terminal part (2345) exclusively forms parallel structures. ${ }^{99}$ These findings already allowed to distinguish the formation of four possible loop-isomers (G-register isomers) for the 2345-conformation. It may be permitted to give a comment at this point from a spectroscopist's or even from a structural biologist's perspective. These two papers have been published from the same authors and claims were made about the structure-based targetability with small-molecule ligands. The false and misleading proposals for structural models without experimental evidence could have been avoided by measuring just a simple CD-spectrum already in the first place. Therefore, this highly cited and nevertheless groundbreaking paper emphasizes the need for at least a basic structural characterization of involved G-quadruplex conformations. 
Initial NMR assignments for the 27-mer long G-quadruplex forming sequence in the $\mathrm{NHE}^{-\mathrm{III}_{1}}$ region have been made by Phan et al. in 2004. ${ }^{111}$ They described two propeller-type, parallel conformations and identified them as conformations 2345 and 1245 (no PDB structures). Yang et al. published the first high-resolution NMR structure for $c M Y C-2345$ (PDB:1XAV, 33 G-register isomer) in early $2005{ }^{460}$ Later in 2005, Phan et al. published a non-canonical G-quadruplex structure with snap-back motif for the 2345 conformation. ${ }^{128}$ A G-to-I mutation in G-tract 3 here is compensated by a replacement with a 3'-terminal G-nucleotide snapped back into the 3'-lower G-tetrad (Figure 21). The NMR structures for $c M Y C-1234(2011)^{461}$ and $c M Y C-1245(2019)^{342}$ then have also been published from the group of D. Yang (Figure 22).

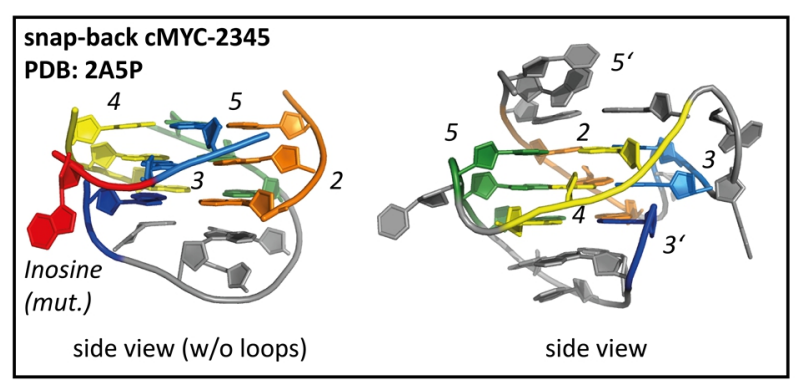

Figure 21: Structure of a parallel snap-back conformation adopted by $c$ MYC-2345 (PDB: 2A5P). ${ }^{128}$ G-tracts are shown coloured (2: orange, 3: blue, 4: yellow, 5: green) loops and capping residues are shown in grey.

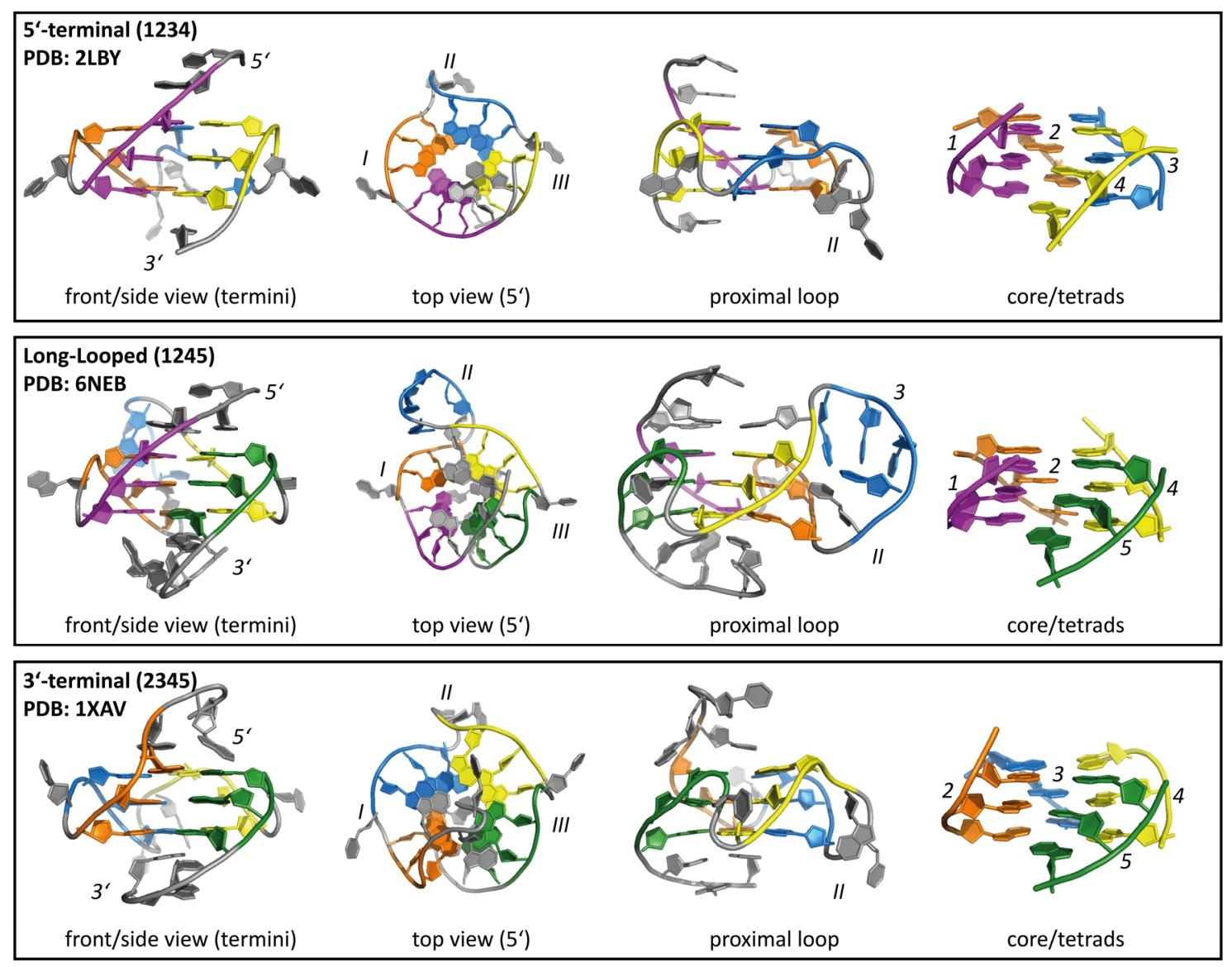

Figure 22: High-resolution NMR-structures of all three relevant G-quadruplex conformations in the Pu27 strand of the cMYC NHE-III ${ }_{1}$. All structures feature an all-parallel strand orientation, but involve different G-tracts in the tetrad/G4-core formation: a 5'-terminal G4 (1234, PDB: 2LBY) ${ }^{461}$, a long-looped G4 (1245, PDB: 6NEB) $)^{342}$ and a 3'-terminal G4 (2345, PDB: $1 \mathrm{XAV})^{460}$. G-tracts are shown in: 1: violet, 2: orange, 3: blue, 4: yellow and 5: green. 
Finally, in 2018 a structure based on X-ray crystallography was published for $c M Y C-2345$ (Figure 23, $\mathrm{RMSD}=2.35 \AA, \mathrm{PDB}=6 \mathrm{AU} 4) .{ }^{522}$ Major deviations for the crystal structure compared to the NMR derived structure concern the 5'- and 3'-terminus. The NMR structure shows a capping coordination that stacks on the top and bottom tetrads, while in the crystal structure the termini are directed outwards. The differences highlight the flexibility of the termini compared to the G4-core. The distal orientation in the crystal structure, however, is most likely caused by packing interactions and dimer stacking. The stabilizing and concealing capping arrangement seen in the NMR structure might be the better reflection of the G4 structure adapted by a DNA oligonucleotide, but the restricted flexibility in a longer sequential context might hamper its formation.
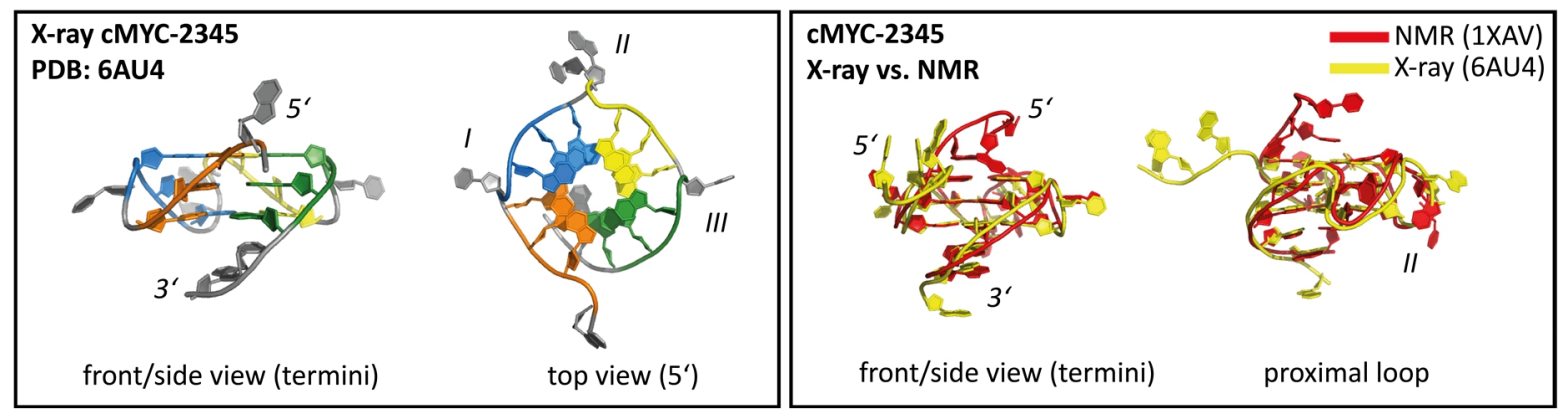

Figure 23: Comparison of the NMR (PDB: 1XAV) 460 and X-ray (PDB: 6AU4) ${ }^{522}$ structures. The NMR-structure shows defined capping structures with stacked residues from the 5'-terminus, while the crystal-structure shows more flexible termini that are directed outwards from the G4-core.

\subsubsection{Thermodynamic Stabilities and Structural Dynamics}

For the four loop-isomers (G-register isomers), a detailed thermodynamic characterization has been reported based on thermal denaturation ( $C D$ melting) and kinetics for folding and unfolding have been derived based on hysteresis. ${ }^{99,100}$ Noteworthy, both studies find melting temperatures $\left(T_{m}\right)$ for each of the single isolated G-register mutants that are lower than for the G-register wt. For a thermodynamic comparison of spare-tire isomers $c M Y C-1245$ and $c M Y C-1234$ also calorimetric studies have been published, ${ }^{112,523}$ which are in line with reported $\mathrm{T}_{\mathrm{m}} \mathrm{s}$ based on CD melting for all reported $c M Y C$ structures. ${ }^{11,342,460,461}$ The plethora of sequence modifications, buffer and DNA concentrations make a detailed comprehensive overview and comparison of all thermodynamic parameters nearly impossible. Furthermore, at physiological $\left[\mathrm{K}^{+}\right], \mathrm{T}_{\mathrm{m}}$ values are typically $>90{ }^{\circ} \mathrm{C}$. Nevertheless, across all published data, a clear trend of melting temperatures for the isolated, stabilized conformations can be drawn as follows:

spare-tire isomers: $1245<1234<<2345<w t$; G-register isomers $(2345): 55<35<53<33<w t$

In general, thermodynamic parameters are typically used to evaluate e.g. the effects of ligand binding. However, such thermodynamic analysis is ambiguous and has to be evaluated carefully to prevent false implications. ${ }^{524}$ This holds particularly true for G-quadruplex forming oligonucleotides that are itself 
prone to polymorphism and structural dynamics. ${ }^{524}$ Already the first structural investigations for the cMYC G-quadruplex revealed the importance of a dynamic interplay of possible conformational isomers. Luciferase expression assays showed a two-fold increase in expression levels for individual (mutated) G-register isomers (five-fold increase for complete G4-destabilization). ${ }^{99}$ A more recent study that investigated the expression levels with a dual-luciferase assay in various cancer cell lines (MCF-7, T47D, MDAMB 231, HeLa, AGS) found an increased level of promoter activity for mutations that restrict the structural polymorphism of the G4 forming element (1234-only: $\sim 1.5$-3 fold, 2345-only: 2-4 fold)..$^{341}$

The role of negative supercoiling has revealed strong implications for a shift of adopted conformations from 2345 towards $1234 .{ }^{456,521}$ Transcriptionally induced negative supercoiling has significant effects in the promoter region up to more than 1000 bases upstream of the TSS. It can affect not only the NHE-III $_{1}\left(-100\right.$ bases to $\left.P_{1}\right)$ but also causes strand separation on the far-upstream element (FUSE, -17000 to $\left.\mathrm{P}_{2}\right){ }^{456}$

Sengupta et al. showed different affinities of G4-binding proteins that interact with the NHE-III (2.4.2) towards different mutations and truncated sequences that preclude the formation of spare-tire isomers. ${ }^{341}$ This study strongly supports a refined model of the transcriptional regulation of the NHE-III 1 that accounts for the spare-tire polymorphism, which is in line with the drastically different affinities of the G4-binding protein nucleolin (2.3.2) towards especially the long-looped conformation $1245 .^{340,342}$

\subsubsection{Targeting the $c M Y C$ G-Quadruplex}

The $c M Y C$ transcription factor has a high significance in cancer progression and is still considered undruggable (2.4.1). Different strategies have been proposed to overcome the main obstacles in therapeutic anti-MYC treatment. ${ }^{478,525-529} c M Y C$ is a prime example for targeting a promoter G-quadruplex with small-molecule ligands as novel strategy in anti-cancer treatment (2.3.4). ${ }^{512,530}$ As discussed above, most common binding motif is stacking on the top and bottom tetrads. For $c M Y C$ a NMR structure of a 2:1 complex with a fluorescent carbazole derivative shows exactly this binding motif (Figure 24). ${ }^{531}$ In this regard it remains challenging to generate binding specificity for a certain G-quadruplex. ${ }^{532}$ Many different scaffolds have evolved e.g. cationic porphyrins ${ }^{533}$, carbazole derivatives, ${ }^{534}$ benzofuranes, ${ }^{535}$ benzothiazoles, ${ }^{536}$ imidazole-benzothiazole conjugates, ${ }^{537}$ thiazole peptides, ${ }^{538}$ triazoles, ${ }^{539}$ 5-nitro-indole derivatives, ${ }^{540}$, binaphtyl-amines, ${ }^{541}$ dansyl-guanosine conjugates $^{542}$ and more complex conjugates like a four-leaf clover diaryl-substituted imidazole/carbazole ${ }^{543}$ and hybrid molecules that link a quadruplex-specific binding site with a duplex minor groove binder (Figure 24). ${ }^{544}$ With this hybrid linker approach, it is possible to generate sequence-specific binding in combination with high G-quadruplex affinity (low $\mathrm{nM}) .^{545}$ 


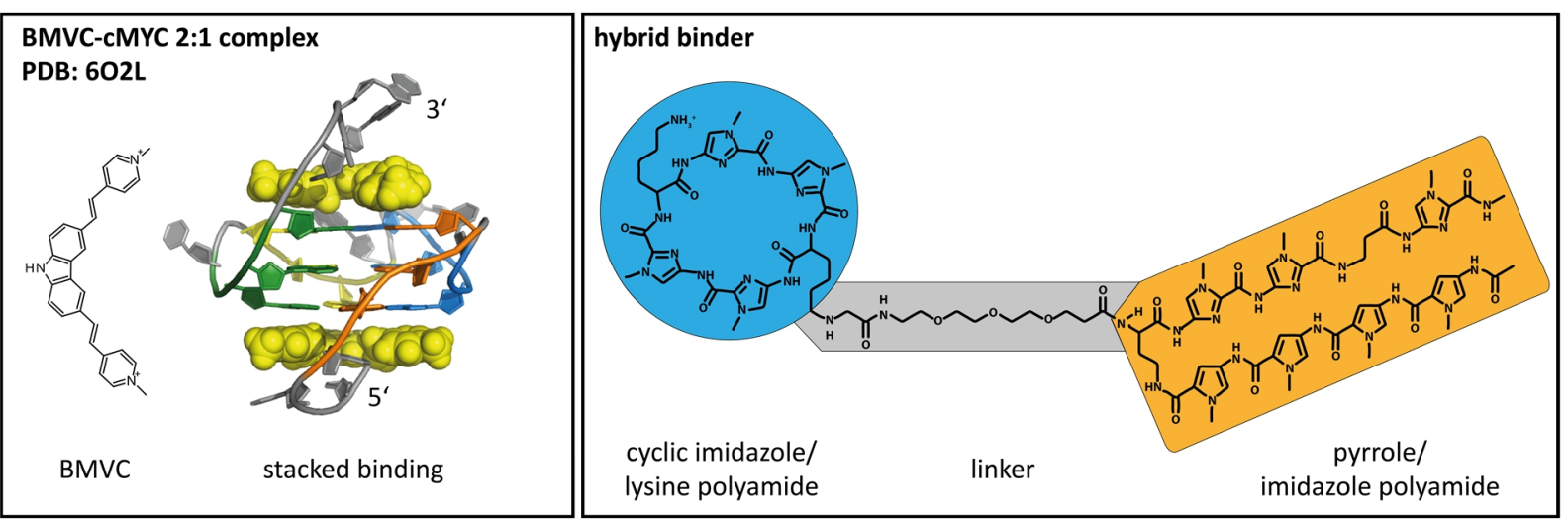

Figure 24: (left) High-resolution NMR-structure (PDB: 602L) 531 of a 2:1 complex of a cMYC G4 with BMVC. (right) Chemical structure of a hybrid G4-binding molecule. The blue part (cyclic imidazole) is able to recognize and bind the G4structure with high affinity. The orange part (pyrrole) can be used to generate sequence specific binding of the contiguous flanking DNA segments. ${ }^{544}$

Methods like rational drug design, ${ }^{540}$ small molecule microarrays ${ }^{535}$ or spectroscopic screening ${ }^{543,546}$ have been used to find these binders and initially evaluate them. One $c M Y C$ G-quadruplex interacting compound (CX-3543 ${ }^{547}$, Quarfloxin) has entered phase II clinical trials, but was finally discontinued due to $M Y C$-independent effects. ${ }^{528,548}$ Recent pre-clinical studies have found GQC- $05^{549}, \mathrm{Cz}^{550}$, IZCZ- $3^{543}$, DC- $34^{551}$, Stauprimidine ${ }^{552}$ and BMH-2 $1^{553}$ as promising drug candidates. ${ }^{529}$ The G4 stabilizer APTO-253 inhibits MYC expression and has entered phase I clinical trials. ${ }^{554-557}$

The compound CX-5461 is a promising possible chemotherapeutic agent that can act as MYC repressor. ${ }^{558}$ The cytotoxic effects have been discussed mainly in RNA polymerase I inhibition, ${ }^{559,560}$ but also in topoisomerase I and II inhibition, ${ }^{561,562}$ activating the DNA damage response; ${ }^{563}$ and CX-5461 is a G4 stabilizer. ${ }^{548,564} \mathrm{~A}$ disturbed $c M Y C$ G4 maintenance is a likely explanation for all of the observed major molecular mechanism for the observed subsidiary molecular mechanisms. CX-5461 has entered phase I clinical trials. ${ }^{565}$

Alternative approaches that aim to target the G4 forming sequence of the NHE-III ${ }_{1}$ use different oligonucleotide (ODN) or peptide strategies. The 12 aa long peptide KR12C (a derivative of the human cathelicidin peptide LL-37) ${ }^{566}$ binds the $c M Y C$ G4 in the nanomolar range in cells and promotes apoptosis. ${ }^{567}$ Triplex-forming oligonucleotides (TFOs) can be used to target the Py27 strand. ${ }^{498-503}$ These strategies benefit from increased lifetimes of the ODN after cellular uptake, presumably due to better nuclease resistance of G4 forming ODNs. ${ }^{502}$ Application of Pu27 to different leukaemia cell lines resulted in up to $90 \%$ reduction of transcription levels and $60 \%$ decrease in protein expression. ${ }^{502}$ Recently, a G-quadruplex-forming RNA motif has been discussed to target nucleolin, which has been suggested as a possible biomarker in lung cancer prognosis. ${ }^{345}$ Strategies like this could be potentially exploited to saturate G4-binding proteins. 
2.4 The cMYC Proto-Oncogene Promoter 


\title{
2.5 NMR Methods to Study Structure and Dynamics in Nucleic Acids
}

\author{
"I have not yet lost that sense of wonder, and of delight, \\ that this delicate motion should reside in all ordinary things around us, \\ revealing itself only to him who looks for it."
}

E.M. Purcell, Nobel lecture $1952^{568}$

\subsubsection{Structure Determination of Nucleic Acids with Natural Abundance Isotopes}

For initial structural screening of G-quadruplex topologies and folding patterns, a combination of NMR-spectroscopic evaluation with $\mathrm{CD}$-spectroscopy is very useful. ${ }^{569} \mathrm{CD}$ spectroscopy provides an easy access to determine the overall folding topology and homogeneity of folded states in G4s that help to analyse and assign the ${ }^{1} \mathrm{H}-\mathrm{NMR}$ spectra. ${ }^{570-574}$ The Gabelica group has also developed a massresolved CD-setup that allows to disentangle polymorphic G4 species. ${ }^{575}$

Experimental approaches to obtain structural information of nucleic acids with nuclear magnetic resonance (NMR)-spectroscopy mainly rely on inter-proton distance restraints from nuclear Overhauser effect spectroscopy (NOESY). ${ }^{576-581}$ A manifold of homo- and heteronuclear NMRexperiments has been developed that help for the assignment of the relevant proton (in particular $\mathrm{N}-\mathrm{H} 1$ imino proton) signals. ${ }^{582-584}$ In addition to that, many NMR-experiments are available that yield information on base pair patterns or structural restraints on dihedral angles (via scalar couplings, $a b b r$. J-couplings) and coordinates for the relative orientation (via residual dipolar couplings, RDC). The strategies and advantages for these experimental approaches have been extensively reviewed. ${ }^{582-584}$ In particular for DNA G-quadruplexes, NMR is the most important method for structure determination at atomic resolution. ${ }^{310,585,586}$ From 277 PDB-structures ("G-quadruplex and DNA", December 2020) 154 (56\%) have been solved by NMR and 123 (44\%) by X-ray crystallography (NMR for: "nucleic acids": $38 \%$; total: $8 \%$ ). However, most heteronuclear 2D-correlated experiment relies on isotope enrichment for the NMR-active (spin-1/2) nuclei ${ }^{13} \mathrm{C}$ and ${ }^{15} \mathrm{~N}$. Isotope labelled RNA (both selectively and uniformly) is easily accessible via in vitro transcription using T7 RNA-Polymerase and the respective isotope labeled NTPs. ${ }^{582,584}$ Preparation of isotope labeled, single stranded DNA via enzymatic methods in appropriate amounts for NMR investigations is not routinely established. For DNA oligonucleotides, isotope labeling is introduced typically via solid-phase oligonucleotide synthesis. Different in vitro methods have been proposed and reported, but did not find widespread application for NMR-studies. ${ }^{587-593}$ Therefore in many cases NMR-spectroscopic studies on DNA G-quadruplexes are limited to samples with a natural abundance ${ }^{594}$ of ${ }^{13} \mathrm{C}(1.1 \%)$ and ${ }^{15} \mathrm{~N}(0.4 \%)$, 
which challenges the applicability of sophisticated, multidimensional heteronuclear experiments due to the low sensitivity.

Figure 25 shows the G4 relevant correlations and connectivities that can be obtained from 2D-NOESY

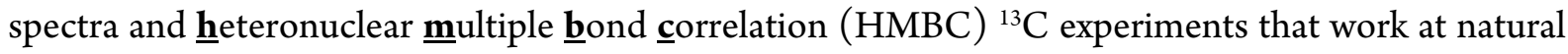
abundance. ${ }^{310,595,596}$ Intra-tetrad $\mathrm{H} 1-\mathrm{H} 1$ and $\mathrm{H} 1-\mathrm{H} 8$ are indicated, expected NOE cross-peak intensity for inter-tetrad correlations are given in Table $2 .{ }^{310}$ Sequential correlations for H8-H1' along the nucleotide chain (5'-3') yield information on the N-glycosidic conformation (syn/anti) and differ for syn-anti, anti-syn or anti-anti steps, Figure 25 shows correlations along an anti-anti step. The relevant $\mathrm{J}_{\mathrm{CH}^{-} \text {-couplings for } \mathrm{H}-\mathrm{C}-\mathrm{H} \text { correlations are given in } \mathrm{Hz} .}^{595,596}$

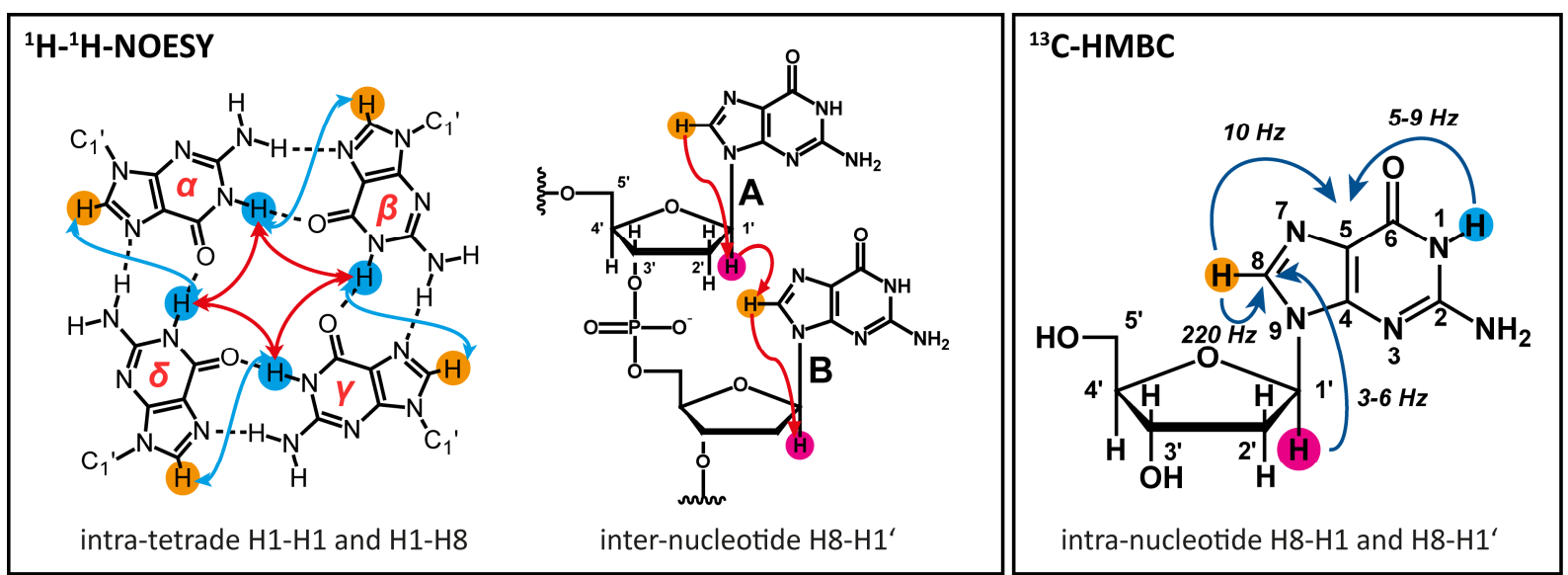

Figure 25: Relevant correlations and connectivities from NMR experiments at natural abundance. NOE connectivities

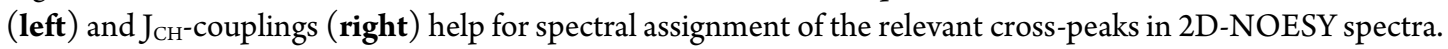

Table 2: Intra- and inter-tetrad NOE-connectivities: expected medium $(3.2-4.6 \AA)$ and weak $(4.6-6.0 \AA)$ cross-peak intensities are indicated. Table was taken from ${ }^{310}$ (PDB entries: 2GKU (hybrid) and 143D (anti-parallel)).

\begin{tabular}{|c|c|c|c|c|c|c|c|}
\hline \multirow{3}{*}{$(\mathrm{n}+1)$} & & \multicolumn{2}{|c|}{$\begin{array}{c}\text { same polarity } \\
\text { (anti-anti) }\end{array}$} & \multicolumn{2}{|c|}{$\begin{array}{l}\text { opposite-polarity } \\
\text { (syn-anti) }\end{array}$} & \multicolumn{2}{|c|}{$\begin{array}{l}\text { opposite-polarity } \\
\text { (anti-syn) }\end{array}$} \\
\hline & & \multicolumn{2}{|c|}{$\mathrm{G}_{\alpha}$} & \multicolumn{2}{|c|}{$\mathrm{G}_{\alpha}$} & \multicolumn{2}{|c|}{$\mathrm{G}_{\alpha}$} \\
\hline & & H1 & $\mathrm{H} 8$ & $\mathrm{H} 1$ & $\mathrm{H} 8$ & $\mathrm{H} 1$ & $\mathrm{H} 8$ \\
\hline \multirow{2}{*}{$\mathrm{G}_{\alpha}$} & H1 & medium & & weak & & medium & \\
\hline & H8 & & weak & & weak & weak & \\
\hline \multirow{2}{*}{$\mathrm{G}_{\beta}$} & H1 & & & & & & \\
\hline & $\mathrm{H} 8$ & & & & & & medium \\
\hline \multirow{2}{*}{$\mathrm{G}_{\psi}$} & H1 & & & weak & & & \\
\hline & H8 & & & & & & \\
\hline \multirow{2}{*}{$\mathrm{G}_{\delta}$} & H1 & weak & weak & medium & & medium & \\
\hline & H8 & & & & & & \\
\hline
\end{tabular}

Guanine amino groups are essential for hydrogen bond formation in G-tetrads (Figure 25). They have rotational freedom around the $\mathrm{C}-\mathrm{N}^{2}$ bond with a rotational frequency between 0.2 and $1.1 \mathrm{kHz}$ (in the millisecond time regime), which affects the linewidths of the signals. Lineshape analysis of amino proton signals from stacked guanines involved in G-quadruplex formation yields useful information on rotation rates and serves as probe for local structural dynamics. ${ }^{597}$ Recently, ${ }^{13} \mathrm{C}$-detected experiments 
("amino-NOESY") have been developed that make use of the amino group dynamics. ${ }^{583,598}$ The sensitivity of these amino-experiments requires ${ }^{13} \mathrm{C}$ isotope labelling, but nevertheless these experiments will be very useful especially for G4s.

\subsubsection{The NMR-Timescale: Methods to investigate Dynamics}

Methods to investigate dynamics with NMR on broad timescales in particular for application on nucleic acids have been extensively reviewed, ${ }^{599-604}$ most recently in a comprehensive overview from the group of K. Petzold ${ }^{605}$.

Spin-relaxation is a non-spontaneous process that is caused by magnetic field fluctuations. Depending on the source of these fluctuations, distinct relaxation mechanisms can be differentiated. The main source of fluctuating fields is molecular tumbling, which slows down with increasing molecular size. $\mathrm{T}_{1}$-relaxation (spin-lattice relaxation, $\mathrm{R}_{1}$ ) is caused by fluctuations only in the $(\mathrm{x}, \mathrm{y})$-direction (static frame), while $\mathrm{T}_{2}$-relaxation (spin-spin relaxation, $\mathrm{R}_{2}$ ) is influenced by fluctuations in any direction. $\mathrm{T}_{1}$ therefore depends on fluctuating fields near the Larmor-frequency $v_{0}$, while $\mathrm{T}_{2}$ is affected by fluctuating fields at any frequency. Relaxation is thus (i) dependent on the magnetic field $\left(\mathrm{B}_{0}\right)$, which correlates with the nuclear Larmor-frequency $v_{0}$ of the respective spins; and (ii) dependent on the molecular size and temperature, which both affect the molecular tumbling. Assuming that molecular motion is isotropic, the rotational correlation time $\tau_{c}$ for a molecule is defined as the average time it takes to rotate through one radian. $\tau_{c}$ therefore is a measure of the average, size-dependent molecular tumbling. ${ }^{606}$ With increasing molecular size (hence increasing $\tau_{c}$ ) $\mathrm{R}_{1}$ reaches an optimum (shortest relaxation time, field dependent) at $v_{0}^{-1} \approx \tau_{c}$, and afterwards gets longer again. $\mathrm{R}_{2}$ instead is steadily decreasing with increasing $\tau_{c}$ and is also affected by chemical exchange and conformational dynamics. The linewidth of NMR signals is proportional to $\mathrm{T}_{2}^{-1}$, thus dynamics in the time regime of $\tau_{c}$ modulate the linewidth and cause severe line broadening. ${ }^{607-609}$

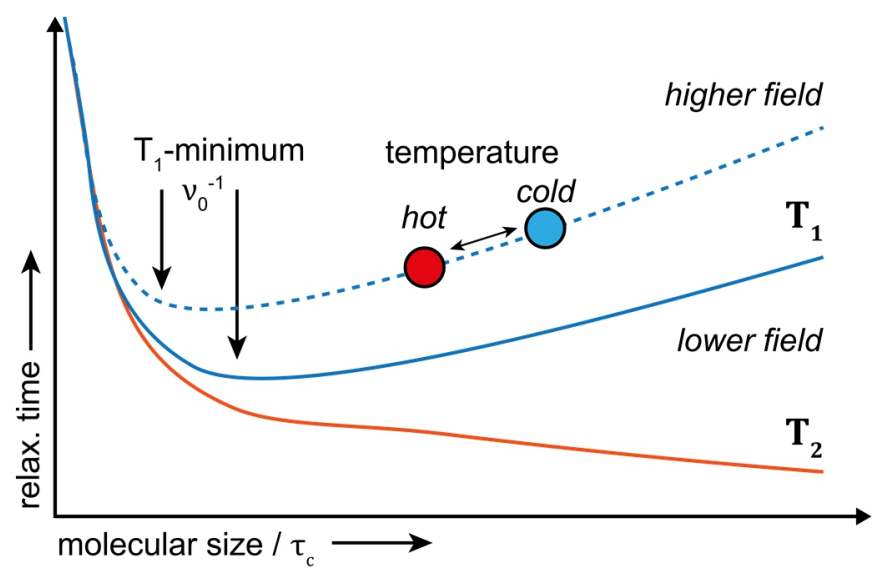

Figure 26: Qualitative trends for relaxation rate constants $T_{1}$ and $T_{2}$ in dependency of the rotational correlation time $\tau_{c}$, magnetic field strength and temperature. $\tau_{c}$ is field-independent, thus increasing magnetic field strength shifts $v_{0}$ (thereby $\Delta \Omega$ ) in relation to $\mathrm{k}_{\mathrm{ex}}$ for a given molecule. Figure was adapted from literature references. ${ }^{607-609}$ 
The NMR-timescale (Figure 27) can be roughly divided in three time regimes for dynamics with the following definition: ${ }^{605}$ In a given system in dynamic exchange between two states $\mathrm{A}$ and $\mathrm{B}$ with the chemical shifts $\Omega_{\mathrm{A}}$ and $\Omega_{\mathrm{B}}$, A and B have a chemical shift difference of $\Delta \Omega$ (1). The exchange rate between $A$ and $B\left(k_{e x}\right)$ is the sum of the forward $\left(k_{A B}\right)$ and reverse $\left(k_{B A}\right)$ rate constant $(2)$.

$$
\begin{gathered}
\mathrm{A}\left(\Omega_{\mathrm{A}}\right) \underset{\mathrm{k}_{\mathrm{A} A}}{\stackrel{\mathrm{k}_{\mathrm{AB}}}{\rightleftarrows}} \mathrm{B}\left(\Omega_{\mathrm{B}}\right) \\
\Delta \Omega=\left|\Omega_{\mathrm{A}}-\Omega_{\mathrm{B}}\right| \\
\mathrm{k}_{\mathrm{ex}}=\mathrm{k}_{\mathrm{AB}}+\mathrm{k}_{\mathrm{BA}}
\end{gathered}
$$

In this system, the time regimes are commonly defined as: slow exchange regime, if $\mathrm{k}_{\mathrm{ex}}<<\Omega$ (seconds and slower); intermediate exchange regime, if $\mathrm{k}_{\mathrm{ex}} \approx \Delta \Omega(\mu \mathrm{s}-\mathrm{ms})$; and fast exchange regime, if $\mathrm{k}_{\mathrm{ex}}>>\Delta \Omega(\mathrm{ps}-\mathrm{ns}) .{ }^{605}$ As discussed above, the time regimes are dependent on $\tau_{\mathrm{c}}$ and do vary, depending on the molecular size. For a given molecular size, the regimes can be slightly shifted with experimental parameters, when varying temperature ( $k_{\text {ex }}$ is temperature-dependent) or magnetic field strength $(\Delta \Omega$ in $\mathrm{Hz}$ is field-dependent).

Dynamic processes faster than $\tau_{c}$ (e.g. bond vibration, libration, angle fluctuation) are averaged out in solution state NMR and do not cause distinguishable states that can be directly detected. Information on dynamics in this time regime can be obtained from $\mathrm{R}_{1}$ and $\mathrm{R}_{2}$ relaxation analysis and NOEs. ${ }^{602,610-}$ ${ }^{612}$ Dynamic processes in the range of/slightly slower than the molecular tumbling rate (supra- $\tau_{c}, \mathrm{~ns}-\mu \mathrm{s}$ ) have for long remained a blind spot for NMR. ${ }^{613}$ This gap now can be probed with experimental approaches that make use of residual dipolar couplings (RDCs) $)^{614,615}$ or cross-correlated relaxation $(\mathrm{CCR})^{616-618}$ rates. In the $\mu$ s- to ms- and s-regime Carr-purcell-Meiboom-Gill (CPMG) ${ }^{619-621}$ sequences and $\underline{\text { Chemical }} \underline{\text { Exchange }} \underline{\text { Saturation }}$ Transfer $(\mathrm{CEST})^{622,623}$ are the most important experimental approaches to investigate dynamics. In the ms to s regime $\underline{\mathbf{E X}}$ change $\underline{\text { Spectroscop}} \underline{\mathbf{Y}}$ (EXSY $)^{624,625}$ can be used to investigate the dynamics of spins that are connected by chemical exchange. In the time regime of seconds and slower, the acquisition of single experiments is faster than the dynamics of the observed system. Here, the information can be obtained in real-time by recording a series of spectra. ${ }^{626-628}$

Independent of the strategy that is used to prepare coherent dynamics for time-resolved NMR measurements (see following chapter) the fast acquisition and sensitivity of the NMR experiments remains a limiting factor. This holds particularly true for multidimensional, mostly heteronuclearcorrelated $\left({ }^{13} \mathrm{C},{ }^{15} \mathrm{~N}\right)$ experiments. Recent advances ${ }^{629}$ in acquisition (SOFAST and BEST) ${ }^{630-633}$ and ultrafast methods ${ }^{634,635}$, sensitivity enhancement (hyperpolarized water) ${ }^{636-640}$ and combinations of ultrafast acquisition with hyperpolarization, ${ }^{611,642}$ have narrowed the timeframe of observable real-time dynamics down to seconds. ${ }^{643,644}$ 


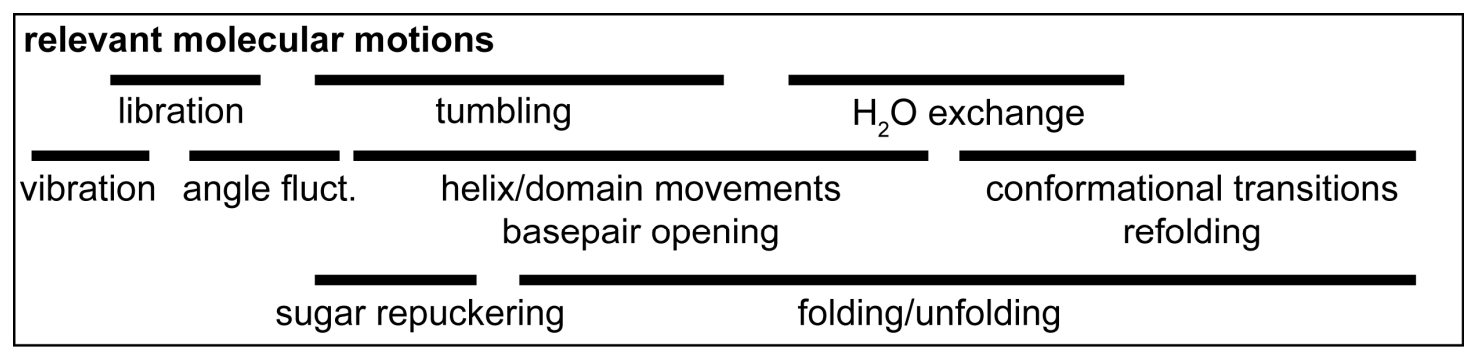

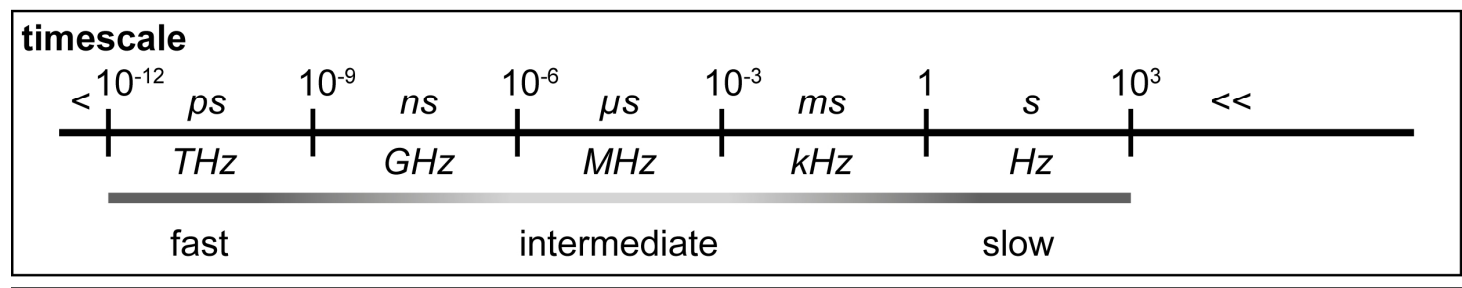

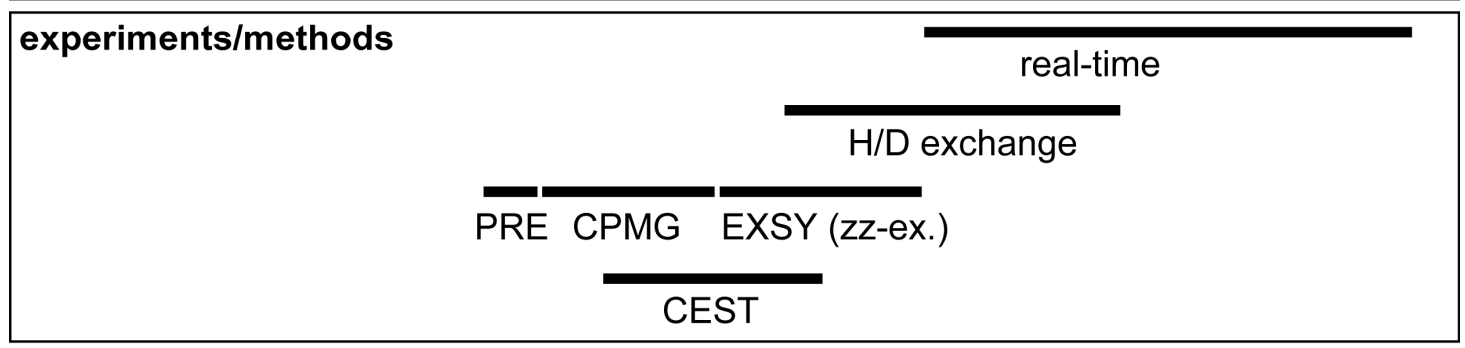

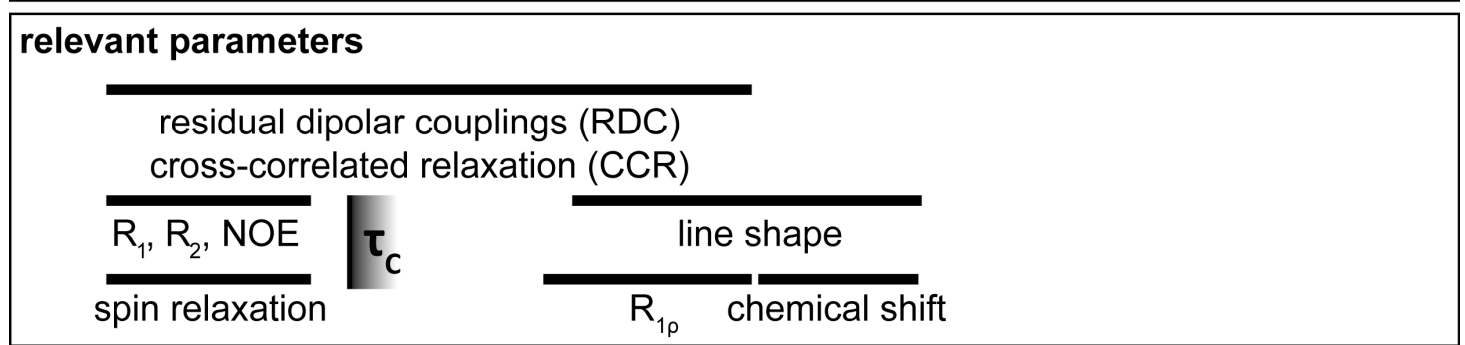

Figure 27: The NMR-timescale ${ }^{602,605,613}$, overview of: the relevant molecular motions for nucleic acids in solution, timescale and frequencies with indicated exchange-regime (fast, intermediate, slow), NMR methods and experiments to probe directly specific dynamic processes, and relevant parameters in NMR experiments that influence and modulate the shape and intensity of NMR signals. $R_{1 \rho}$ is defined as relaxation in the rotating frame, measurement of $R_{1 \rho}$ is strictly rather a method than a parameter. ${ }^{65,646}$

\subsubsection{Real-Time NMR: Non-Equilibrium Dynamics}

Two different types of dynamics shall be differentiated in this chapter: reversible and irreversible events. The folding of nucleic acids follows an irreversible trajectory, while steady-state refolding or e.g. domain motions have reversible trajectories. In general, NMR spectroscopy detects a large ensemble of spins in concentrated samples. This is different to single-molecule methods like Fluorescence $\underline{R}$ esonance Energy Transfer (smFRET) ${ }^{647,648}$ microscopy, where single events can be detected. This allows for the observation of both kind of dynamics. In solution state NMR, all dynamic changes within a molecule are ensemble averaged. Time-resolved studies of both kind of structural dynamics thus require coherent progression of the underlying molecular motions. To create a coherent evolution, different ways to prepare non-equilibrium, excited or meta-stable states are feasible. Timeresolved NMR typically tracks the relaxation of these states back to their thermodynamic equilibrium or steady states. While this thesis was written, a review with contributions from me (the author of this thesis) was published as preprint. ${ }^{628}$ 


\section{Temperature or pressure change}

The intensive properties temperature $(\mathrm{T})$ or pressure $(\mathrm{p})$ influence the structural and physicochemical properties of biomacromolecules, which makes them suitable variables to investigate thermodynamic and kinetic parameters. ${ }^{649}$ High pressure or temperature results in e.g. structural changes (pressurerelated stabilization or unfolding; or thermal denaturation $)^{183,650-652}$, or disassembly/disaggregation of molecular complexes ${ }^{653}$. A steady application of high pressures ${ }^{654-656}$ (up to $3 \mathrm{kbar}^{657}$ ) forces biomolecular systems to populate excited states and shift conformational equilibria, which yields useful, otherwise hidden ${ }^{658}$ information. ${ }^{184,659,660}$

Technical setups for rapid in situ changes in temperature (T-jump: microwave-heating ${ }^{661,662}$, laserinduced (MAS ssNMR) ${ }^{663,664}$, rf-heating ${ }^{665,666}$ or capacitively coupled ${ }^{667}$ ) or pressure (p-jump $\mathbf{p}^{668-673}$ ) have been reported that can be used to investigate the relaxation back to thermodynamic equilibrium. Only a limited number of T-jump NMR experiments has been reported for biomolecules. Akasaka et al. have pioneered an experiment for temperature-induced folding of RNase A at low $\mathrm{pH}\left(\Delta \mathrm{T}=16^{\circ} \mathrm{C}\right.$ in $6 \mathrm{~s}) .{ }^{674}$ More recently, application of T-jump NMR for protein folding has been demonstrated for the investigation of cold-denatured Barstar $\left(\Delta \mathrm{T}=20^{\circ} \mathrm{C}\right.$ in $\left.500 \mathrm{~ms}\right) .^{675}$ The Bax group used p-jump NMR experiments to study ubiquitin protein folding with pressure jumps up to $2.5 \mathrm{kbar} .{ }^{669,676,677}$ Numerous studies also applied high-pressure and p-jump experiments to study $\beta$-amyloid aggregation. ${ }^{653,678-680}$

\section{Rapid-mixing}

Rapid-mixing measurements allow the investigation of kinetics that can be initiated by different means. ${ }^{681}$ Yushmanov and Furó reported on a rapid-mixing setup with stopped-flow design ${ }^{682}$; a device for rapid-mixing of components within a NMR-sample has been reported by Mok et al. ${ }^{633}$ and has been refined recently ${ }^{684}$. Using rapid-mixing, protein (re-)folding has been investigated e.g. after dilution of a chemically denatured (guanidinium chloride) state $^{685}$ (vice versa for unfolding ${ }^{686}$ ) or $\mathrm{pH}-$-change ${ }^{687,688} \cdot{ }^{689-691}$ Folding of nucleic acids after rapid-mixing has been investigated e.g. for RNA riboswitches by inducing their formation with addition of their specific ligand, ${ }^{692}$ for ribozymes by

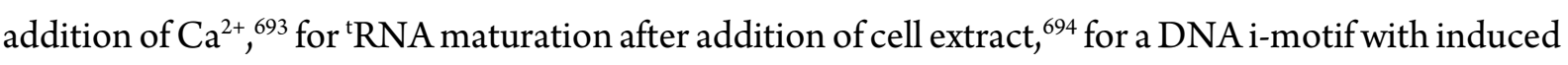
$\mathrm{pH}$-change ${ }^{76}$ and for a telomeric DNA G-quadruplex by inducing its formation with addition of $\mathrm{K}^{+} .{ }^{144}$

\section{Photochemical trapping}

Instead of rapid mixing, a binding event can also be initiated after releasing a trapped (caged) ligand with light. This has been demonstrated for ligand-induced riboswitch folding after in situ photochemical release of photocaged hypoxanthine. ${ }^{695}$ For protein folding a similar approach has been reported, utilizing a light-trigger; therefore a photolabile nitrophen chelator has been used to release $\mathrm{Ca}^{2+}$ and study the ion-induced refolding of $\alpha$-lactalbumin. ${ }^{696,697}$ 


\subsubsection{Conformational Selection with Photolabile Protecting Groups}

Light is an excellent trigger to obtain selective (spatio-) temporal control over chemical processes in a given experimental setup. Different chemical strategies have been developed to make use of light absorption as a versatile tool for reaction control. ${ }^{698-701}$ These tools can be reversible photoswitches ${ }^{702-705}$ that react to light (e.g. in $\mathrm{E} / \mathrm{Z}$ isomerization along a $-\mathrm{N}=\mathrm{N}$ - double bond in azobenzene dyes or a $-\mathrm{C}=\mathrm{C}$ double bond within stilbene chromophores) or irreversible photocleavable moieties (photosensitive/photolabile protecting groups, PPGs) ${ }^{706}$ (Figure 28). As they help to block molecular interactions and suppress reactivity at distinct sites in a molecule temporarily, photolabile protecting groups have been named photocages; their photolytic "release" hence is called uncaging.

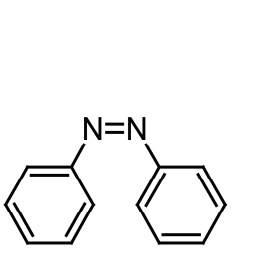

(Z)-azobenzene

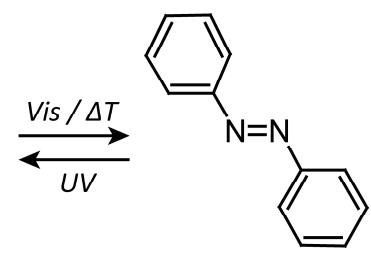

(E)-azobenzene<smiles>[R]c1cc([C@@H]([R])[AlH2])c([N+](=O)[O-])cc1[R]</smiles>

ortho-nitrobenzyl group

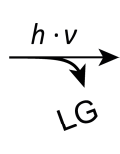

nitroso-ketone/aldehyde irreversible photocaging

photoswitching

Figure 28: Paradigm reaction schemes for reversible (photoswitches, left) and irreversible (photosensitive protecting groups/photocages, right) interactions with light. Azobenzene and ortho-nitrobenzyl derivatives have established the

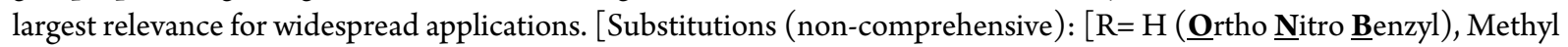

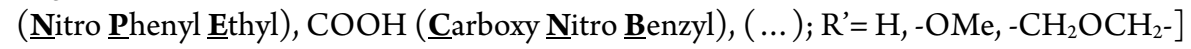

Photolabile protecting groups for use in nucleic acid/sugar chemistry have first been reported in 1977. ${ }^{707}$ This very first study already introduced ortho-nitrobenzyl (ONB) (Figure 28) as photocleavable group to block cAMP (Figure 29). The ONB scaffold is synthetically easy accessible and can be fine-tuned for light-stability or wavelength specificity, hence it has been commonly established in widespread applications. After photocleavage ONB $(\mathrm{R}=\mathrm{H})$ yields nitrosoaldehydes, nitro-phenyl-ethyl (NPE, $\mathrm{R}=\mathrm{Me}$ ) yields nitrosoketones, which are less cell-toxic. NPE can also be deprotected faster than ONB and therefore is typically the cage of choice.

Photocages can be attached to backbone phosphate groups ${ }^{708-710}$, nucleobases ${ }^{29,711-716}$ or ribose rings at the $2^{\prime}-\mathrm{OH}^{717,718}$; also photocleavable linkers have been introduced both in the phosphate backbone $^{719,720}$ and at nucleobases ${ }^{721}$. All these strategies (Figure 29) can be used to block molecular interactions and thereby influence the structure and functions of DNA/RNA.

Photocaging of nucleobases prevents base pairing interactions at the Watson-Crick and/or Hoogsteen interface in two ways: (i) sterically and (ii) by locking the $\mathrm{T} / \mathrm{U}: \mathrm{N}^{3}-\mathrm{C}^{4}-\mathrm{O}^{4}$ and $\mathrm{G}: \mathrm{N}^{1}-\mathrm{C}^{6}-\mathrm{O}^{6}$ bond in an enolic form (-N=C-O-), which alters the hydrogen-bond donor/acceptor pattern. This approach has been used to destabilize certain secondary or tertiary conformations in RNA and DNA oligonucleotides. . $^{29,712,713,716}$ 

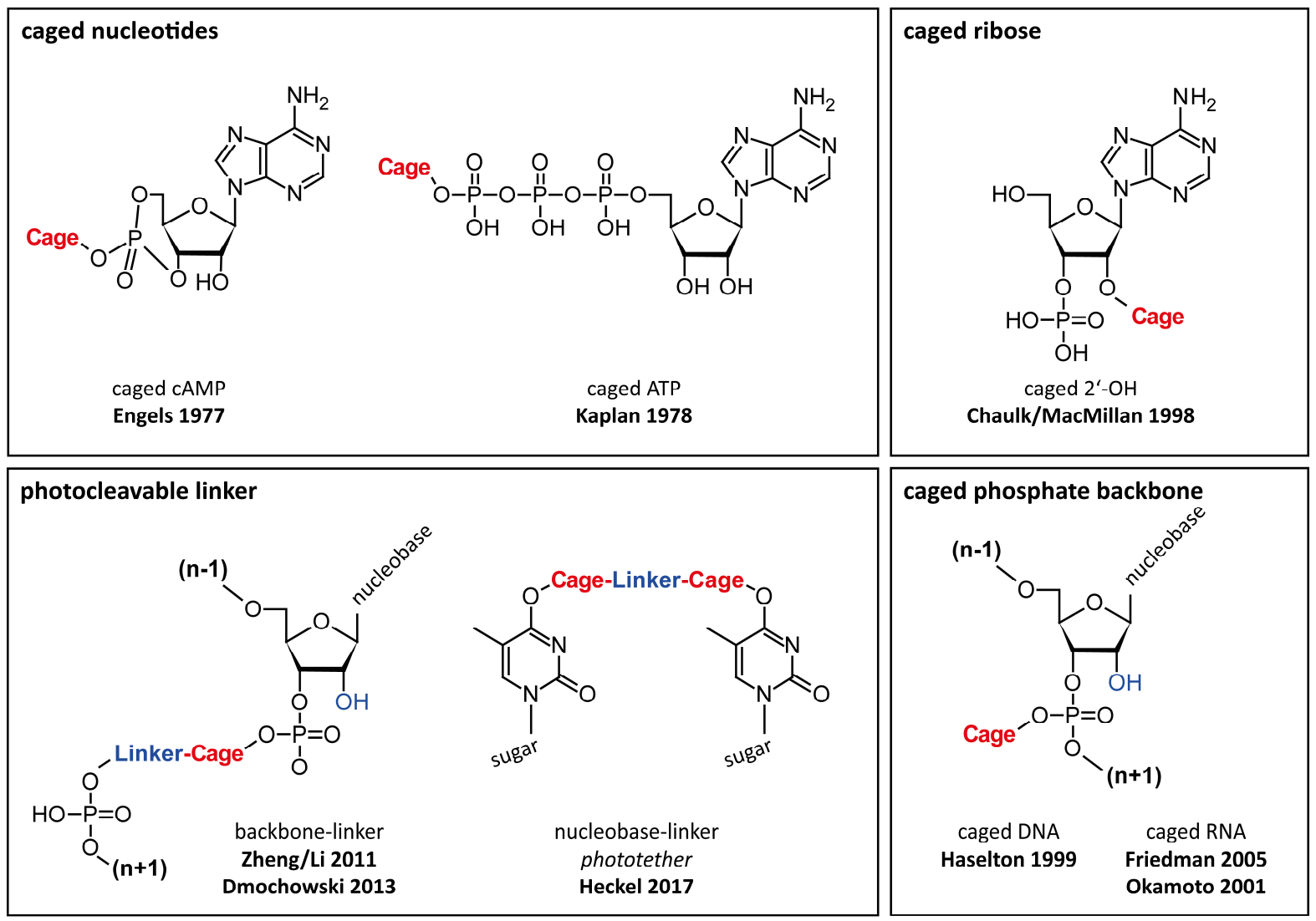

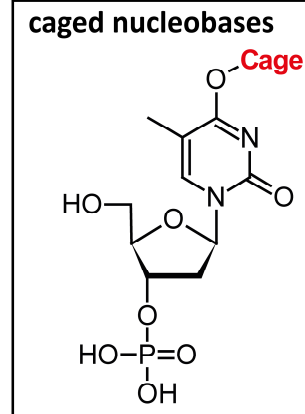

caged dT Heckel 2004

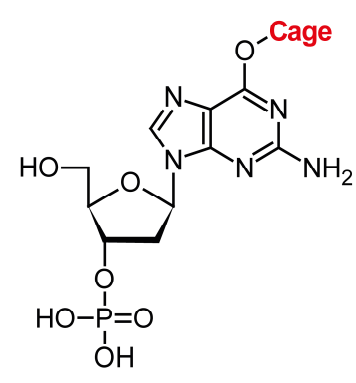

caged dG Mayer/Heckel 2005

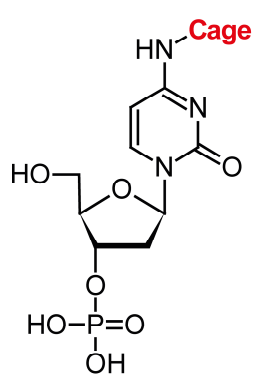

caged dC Heckel/Mayer 2006

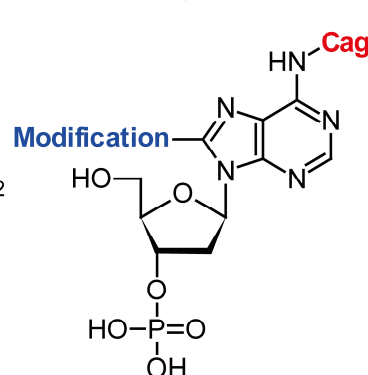

caged dA Perrin 2004<smiles>CC(C)(C)Oc1ccn(C2OC3OC(C2O)C(OP(=O)(O)O)C3O)c(=O)n1</smiles>

caged $U$

Wenter 2006

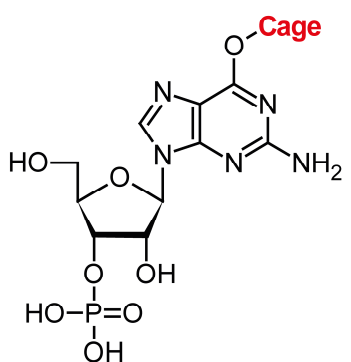

caged G Wenter 2005<smiles>CC(Cl)(Cl)Nc1ccn(C2OC3(CO)OC(OP(=O)(O)O)C2C3O)c(=O)n1</smiles>

caged C

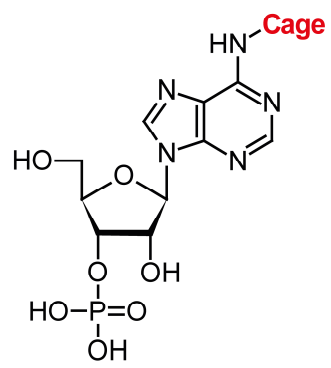

caged A
Höbartner 2005

Figure 29: Overview of caging strategies for DNA/RNA nucleosides: early approaches of caged-ATP ${ }^{707,722}$, 2'-caged ribose $^{717,718}$, caged phototethers ${ }^{719-721}$, caged phosphate backbone $e^{708-710}$ and the entire set of caged nucleotides for $\mathrm{DNA}^{711,714-716}$ and RNA $712,713,723$.

Blocking biological function does not require a complete distortion of the functional fold of nucleic acids, but can be achieved even within their native conformation and single photocages can be sufficient to block e.g. ribozyme cleavage. ${ }^{717}$ A sufficiently large destabilization with reasonably sparse 
incorporation of photolabile protecting groups that prevents secondary structure formation of oligonucleotides has been first demonstrated for a thrombin binding aptamer (TBA, Figure 30). ${ }^{29}$ TBA adopts a two-tetrad G-quadruplex structure in its binding competent state ${ }^{29}$; suppression of a threetetrad G-quadruplex formation then also has been achieved for an oligonucleotide from a telomeric sequence ${ }^{716}$. However, thermodynamically, photocaging base pair interactions only causes local structure disturbance ${ }^{724-726}$ and a complete suppression of secondary or even tertiary conformations remains challenging. ${ }^{713}$ In this context, alternative strategies that are making use of cyclized oligonucleotides via phototethers are powerful improvements for conformational caging (Figure 30). ${ }^{721}$
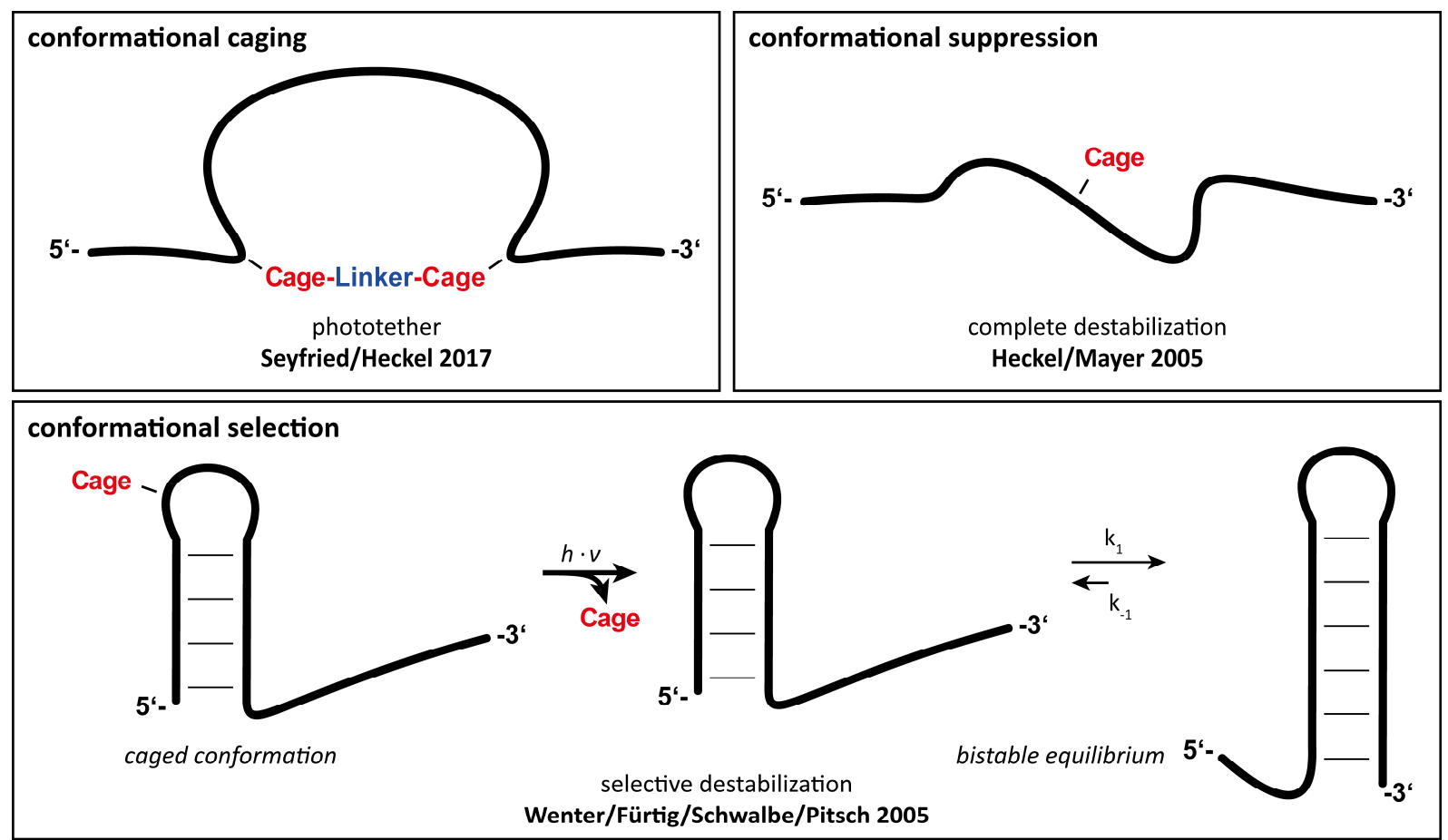

Figure 30: Different caging strategies for DNA/RNA oligonucleotides: conformational caging using phototethers ${ }^{721}$, conformational suppression or destabilization ${ }^{29,716}$ and conformational selection or trapping with specific site-directed, local destabilization ${ }^{712}$.

The flat conformational energy-landscapes for nucleic acids (2.2.1) often causes the formation of competing conformations $(2.1 .2,2.1 .3)$. A well-studied example $\mathrm{e}^{627,712,723,727-729}$ for this are two 20 -mer RNA oligonucleotide sequences $\left(\mathrm{I}^{730}, \mathrm{II}^{731}\right)$ that can each form a bistable two-state equilibrium system of two coexisting hairpin conformations. The two conformations are separated by only a small enthalpy difference $\left[\left(\Delta \mathrm{H}_{\mathrm{I}}=-5.9 \mathrm{kcal} \cdot \mathrm{mol}^{-1}\right)^{712} ;\left(\Delta \mathrm{H}_{\mathrm{II}}=-5.9 \mathrm{kcal} \cdot \mathrm{mol}^{-1}\right)^{723}\right]$ and incorporate different residues into base pair formation. In their first of its kind publication, Wenter et al. ${ }^{712}$ selected a single conformation by attaching a photocage to a residue that is in the loop for one conformation, but in the base paired stem for the other conformation. This selective destabilization (Figure 30) led to a homogenous population of a single folded conformation. A quantitative photolysis enabled a complete relaxation to the equilibrium populations. 
General Introduction 


\section{Materials and Methods}

\subsection{DNA Oligonucleotides and Sample Preparation}

All used DNA oligonucleotides have been HPLC-purified, desalted via centrifugation over a vivaspin (Sartorius) centrifugal concentrator with $1 \mathrm{kDa}$ molecular-weight cut-off, lyophilized and dissolved in $\mathrm{ddH}_{2} \mathrm{O}$. Salt-free samples for $\mathrm{K}^{+}$-induced folding have been repeatedly heated to $95^{\circ} \mathrm{C}$ and diluted with $5 \mathrm{M} \mathrm{LiCl}$ solution. Concentrations have been determined with $\mathrm{UV} /$ vis absorption at $260 \mathrm{~nm}$. Molar extinction coefficients $\left(\varepsilon_{260}\right)$ were calculated using a nearest-neighbour method. Unmodified DNA oligonucleotides have been purchased by eurofins Genomics, Ebersberg (Germany) and HPLCpurified by the manufacturer. Modified (photocaged) DNA oligonucleotides have been synthesized in the group of Prof. A. Heckel. Dean-Paulos Klötzner ( ${ }^{\mathrm{DK}}$, synthesis 1) or Anja Blümler ( ${ }^{\mathrm{AB}}$, synthesis 2).

\subsubsection{Overview of DNA Oligonucleotides}

Table 3: DNA oligonucleotide sequences used for the experiments presented in this thesis. (X= (R)-NPE-dG), (Synthesis: $\mathrm{DK}=$ Dean-Paulos Klötzner, $\mathrm{AB}=$ Anja Blümler $),(\mathrm{f}=$ caged in folded state, uf = caged in unfolded state $), \mathrm{G}$-to-T mutations in bold, G-tracts underlined.

\begin{tabular}{|c|c|c|c|c|}
\hline Origin & Length & Conformation & Modification & Sequence \\
\hline \multirow{21}{*}{$c M Y C$} & \multirow{5}{*}{18} & $2345-53$ & -- & AGGGTTGGGAGGGTGGGT \\
\hline & & $2345-33$ & -- & AGGGTGGGTAGGGTGGGT \\
\hline & & $2345-\mathrm{X} 3$ & -- & AGGGTGGGGAGGGTGGGT \\
\hline & & 2345-wt & -- & AGGGTGGGGAGGGTGGGG \\
\hline & & 1234 & -- & TGGGAGGGTTGGGAGGGT \\
\hline & \multirow{4}{*}{22} & wt-53 & -- & TGGGAGGGTTGGGAGGGTGGGT \\
\hline & & 1234 & -- & TGGGAGGGTTGGGAGGGTTTTT \\
\hline & & 1245 & -- & TGGGAGGGTTTTTAGGGTGGGT \\
\hline & & 2345 & -- & TTTTAGGGTTGGGAGGGTGGGT \\
\hline & \multirow{6}{*}{18} & $2345-53(f)$ & $1 \mathrm{xNPE} \mathrm{EK}^{\mathrm{DK}}$ & AGGGTTGGGAGGGTGGGT \\
\hline & & $2345-33(f)$ & $1 \times N P E^{D K}$ & AGGGTGGGTAGGGTGGGT \\
\hline & & $2345-53^{\prime}(\mathrm{f})$ & $2 \mathrm{xNPE}^{\mathrm{DK}}$ & AGGGTXGGGAGGGTGGGX \\
\hline & & $2345-53$ (uf) & $3 \mathrm{xNPE}^{\mathrm{DK}}$ & AGGGTTGXGAGXGTGXGT \\
\hline & & $2345-\mathrm{X} 3$ (uf) & $3 \times \mathrm{NPE}^{\mathrm{AB}}$ & AGGGTGGXGAGXGTGXGT \\
\hline & & 1234 (uf) & $3 x N P E^{A B}$ & TGGGAGXGTTGXGAGXGT \\
\hline & \multirow{6}{*}{22} & $1234(f)$ & $1 \times \mathrm{NPE}^{\mathrm{AB}}$ & TGGGAGGGTTGGGAGGGTGXGT \\
\hline & & $1245(\mathrm{f})$ & $1 \mathrm{xNPE} \mathrm{AB}^{\mathrm{AB}}$ & TGGGAGGGTTGXGAGGGTGGGT \\
\hline & & wt-53 (uf) & $3 \times \mathrm{XPE}^{\mathrm{AB}}$ & TGGGAGGGTTGXXAGX \\
\hline & & 1234 (uf) & $2 \mathrm{xNPE}^{\mathrm{AB}}$ & TGGGAGGGTTGXGAGXGTTTTT \\
\hline & & 1245 (uf) & $3 \mathrm{xNPE} \mathrm{E}^{\mathrm{AB}}$ & TGGGAGXGTTTTTAGXGTGXGT \\
\hline & & 2345 (uf) & $3 \mathrm{xNPE} \mathrm{E}^{\mathrm{AB}}$ & TTTTAGGGTTGXGAGXGTGXGT \\
\hline \multirow{4}{*}{ hTERT } & \multirow{4}{*}{20} & 53 , parallel (f) & -- & A $\underline{\mathbf{T} G G G A G G G T C T G G G A G G G C}$ \\
\hline & & 35, hybrid (f) & -- & AGGGTATGGGCTGGGAGGGC \\
\hline & & 53 , parallel (f) & $2 \mathrm{xNPE}^{\mathrm{DK}}$ & A $\overline{\mathbf{X G G G} A G G G \mathbf{X}}$ CTGGGAGGGC \\
\hline & & 35, hybrid (f) & $2 \mathrm{xNPE} \mathrm{E}^{\mathrm{DK}}$ & A $\overline{\text { GGGX }}$ A $\overline{\mathbf{X G G G}}$ CT $\overline{\text { GGGA }} \overline{\text { GGGC }}$ \\
\hline
\end{tabular}




\subsubsection{Synthesis of Photocaged DNA Oligonucleotides}

The protocols presented here in the following are kindly provided by the respective synthesist that have conducted the synthesis as collaboration partners within the presented projects. The protocols are taken from the Supporting Information of Grün et al. $\mathbf{2 0 2 0}^{732}$ and Grün et al. 2021 ${ }^{733}$, where also further details and analytics can be found. In general, chemical synthesis is an fascinating process. ${ }^{74,735}$

\section{Synthesis 1, performed and reported by Dean-Paulos Klötzner (Grün et al. 2020)}

The reactions presented were performed under argon atmosphere using dry solvents. NMR analysis of new compounds were performed on Bruker AV500 and DRX600 MHz spectrometers. Assignment of proton chemical shifts was done via ${ }^{1} \mathrm{H}-{ }^{1} \mathrm{H}$-COSY experiments. For flash chromatography silica gel 60 by Macherey-Nagel was used. Compound 1 was prepared according to Mayer et al. ${ }^{716}$.

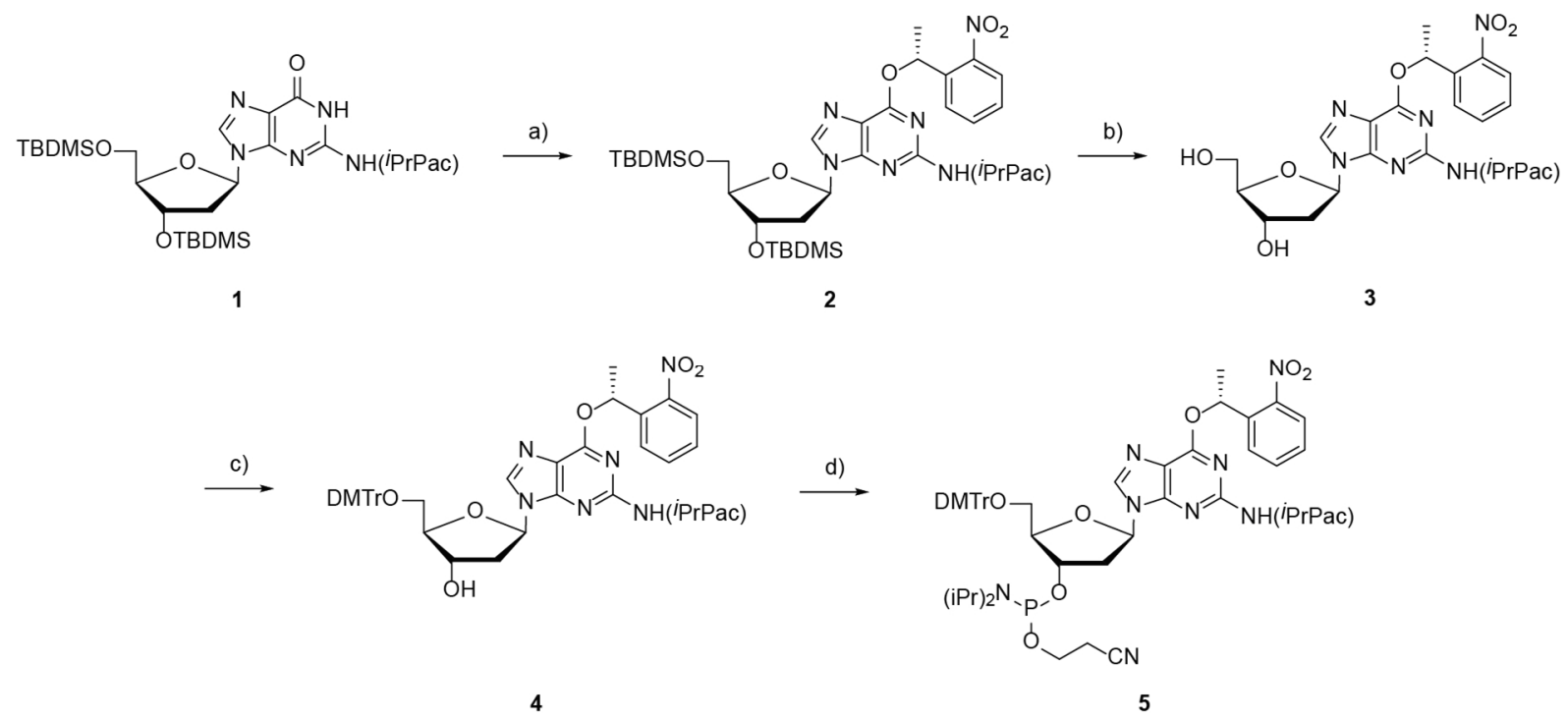

Scheme 1: Synthesis of ${ }^{(R)-\mathrm{NPE}} \mathrm{dG}$ phosphoramidite. a) (S)-1-(2-Nitrophenyl)ethanol (prepared as described in the literature $\left.{ }^{736}\right), \mathrm{PPh}_{3}, \mathrm{DEAD}$ (40 wt\% in toluene), THF, 65\%; b) TBAF, HOAc, THF, 78\%; c) 4,4'-Dimethoxytrityl chloride, pyridine, $82 \%$; 2-Cyanoethoxy- $\mathrm{N}, \mathrm{N}$-diisopropylaminochlorophosphine, (iPr) ${ }_{2} \mathrm{NEt}$, DCM, $71 \%$.

\section{Synthesis of compound 2 (65\% yield):}

$4.09 \mathrm{~g}$ DEAD solution ( $40 \mathrm{wt} \%$ in toluene, $9.39 \mathrm{mmol}, 1.5$ eq.) was added dropwise to a solution of $4.21 \mathrm{~g}$ of compound 1 (6.26 mmol, 1 eq.), $1.15 \mathrm{~g}(S)$-1-(2-nitrophenyl)ethanol (6.89 mmol, 1.1 eq.) and $2.46 \mathrm{~g} \mathrm{PPh}_{3}$ (9.39 mmol, 1.5 eq.) in $30 \mathrm{~mL}$ dry THF. The solution was stirred for 60 minutes at room temperature, concentrated and diluted with $200 \mathrm{~mL}$ DCM and $200 \mathrm{~mL}$ brine. The aqueous layer was extracted with $100 \mathrm{~mL}$ DCM $(2 \mathrm{x})$. The combined organic phases were dried over $\mathrm{MgSO}_{4}$. The solvent was evaporated and the residue purified via column chromatography (cyclohexane/EtOAc $3: 1 \rightarrow 1: 1)$ to give 2 as a yellowish foam.

\section{Synthesis of compound 3 (78\% yield):}

$3.3 \mathrm{~g}$ of compound 2 ( $4.02 \mathrm{mmol}, 1$ eq.) were dissolved in $40 \mathrm{~mL}$ dry THF. $1.38 \mathrm{~mL}$ Acetic acid (24.11 mmol, 6 eq.) and $12.06 \mathrm{~mL}$ TBAF solution ( $1 \mathrm{M}$ in THF, $12.06 \mathrm{mmol}, 3$ eq.) were added. The reaction mixture was stirred at room temperature for 23 hours. The solvent was evaporated and the residue purified via column chromatography $(\mathrm{DCM} / \mathrm{MeOH}$ 99:1 $\rightarrow$ 92:8) to give 3 as a yellowish foam. 


\section{Materials and Methods}

\section{Synthesis of compound 4 ( $82 \%$ yield):}

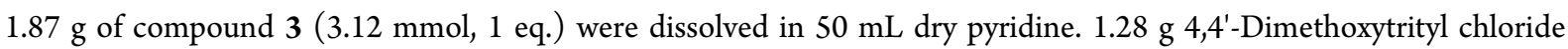
( $3.79 \mathrm{mmol}, 1.2$ eq.) were added in portions. The reaction mixture was stirred at room temperature for 3 hours. The solvent was evaporated and the residue diluted with $200 \mathrm{~mL}$ DCM and washed with $200 \mathrm{~mL}$ saturated sodium bicarbonate solution. The aqueous layer was extracted with $100 \mathrm{~mL} \mathrm{DCM}(2 \mathrm{x})$. The combined organic layers were dried over $\mathrm{MgSO}_{4}$ and evaporated. The residue was purified via column chromatography (DCM/MeOH/Et $\left.{ }_{3} \mathrm{~N} 98: 1: 1\right)$ to give 4 as a yellowish foam.

\section{Synthesis of compound 5 ( $71 \%$ yield):}

$1 \mathrm{~g}$ of compound 4 (1.12 mmol, 1 eq.) and $950 \mu \mathrm{L}(i \operatorname{Pr})_{2} \mathrm{NEt}(5.59 \mathrm{mmol}, 5$ eq.) were dissolved in $15 \mathrm{~mL}$ dry DCM. $529 \mathrm{mg}$ 2-Cyanoethoxy- $N, N$-diisopropylaminochlorophosphine ( $2.23 \mathrm{mmol}, 2 \mathrm{eq}$.) were added. The reaction mixture was stirred at room temperature for 1 hour. The solution was diluted with $150 \mathrm{~mL} \mathrm{DCM}$ and washed with $150 \mathrm{~mL}$ saturated sodium bicarbonate solution. The aqueous layer was extracted with $100 \mathrm{~mL} \mathrm{DCM}(2 \mathrm{x})$. The combined organic layers were dried over $\mathrm{MgSO}_{4}$ and evaporated. The residue was purified via column chromatography (cyclohexane/EtOAc 4:1 $\rightarrow$ 1:1 $\rightarrow$ 1:2) to give 5 as a yellowish foam. The column was packed with cyclohexane/EtOAc $4: 1+0.5 \% \mathrm{Et}_{3} \mathrm{~N}$ while the eluent was prepared without $\mathrm{Et}_{3} \mathrm{~N}$.

\section{Oligonucleotide synthesis}

Photocaged oligonucleotides were synthesized on an Äkta Oligopilot in $18 \mu \mathrm{mol}$ (S1), $16 \mu \mathrm{mol}$ (S2), $13 \mu \mathrm{mol}$ (S3), $16 \mu \mathrm{mol}(\mathrm{S} 4)$ and $19 \mu \mathrm{mol}$ (S5) scales. The following reagents were purchased from Sigma Aldrich: DMT-dG-(tac)-; DMT-dA-(tac)-; DMT-dC-(ac)-; and DMT-dT phosphoramidites and Fast Deprotection Cap 2 containing 4-tert-butylphenoxyacetic anhydride. 5-(Ethylthio)-1H-tetrazole was used as activator. 500 Å DMT-dT-CPG from Glen Research, 500 Å DMT-dC-(tac)-CPG from Millipore, 500 Å CUTAG CPG and 500 Å DMT-dG-(tac)-CPG from Sigma Aldrich were used. All caged oligonucleotides were synthesized DMTr-ON. Deprotection of S1, S2, S4 and S5 was performed using $6.5 \mathrm{~mL} \mathrm{NH}_{4} \mathrm{OH}$ for 1.5 hours at $65^{\circ} \mathrm{C}$ while $\mathrm{S} 3$ was deprotected using $6.5 \mathrm{~mL} t$ - $\mathrm{BuNH}_{2}$ :water $(1: 3)$ for 4 hours at $60^{\circ} \mathrm{C}$. After deprotection the solvent was evaporated and the crude product purified via RP-HPLC using a MultoKrom 100 - 5 C18 column (dimensions: $20 \cdot 250 \mathrm{~mm}$, gradient: 5-40\% MeCN in 0.1 M TEAA buffer pH $=7$ in $44 \mathrm{~min}$, flow: $10 \mathrm{~mL} / \mathrm{min}$ ) on an HPLC from Young Lin Instruments with SP930D pumps and a UV730D detector. After evaporation of the solvent, the DMTr group was removed with $80 \% \mathrm{HOAc}$ for $20 \mathrm{~min}$ at room temperature. The resulting oligonucleotides were purified again via RP-HPLC using the conditions mentioned before.

\section{Synthesis 2, performed and reported by Anja Blümler (Grün et al. 2021)}

All reactions were performed under argon atmosphere using dry solvents purchased from Acros Organics or Sigma Aldrich. Reagents were purchased from Acros Organics, Sigma Aldrich, ChemPur, TCI, Alfa Aesar or Carbosynth and used without further purification. For flash chromatography the used silica gel was purchased from Macherey-Nagel (particle size: 40-63 $\mu \mathrm{m}$ ), solvents were of technical grade. NMR spectra were recorded on Bruker DPX250, AV400 and AV500 instruments at ambient temperature. The synthesis of the (R)-NPE protected 2'-deoxyguanosine phosphoramidite was performed according to literature procedure. ${ }^{716,736}$ 


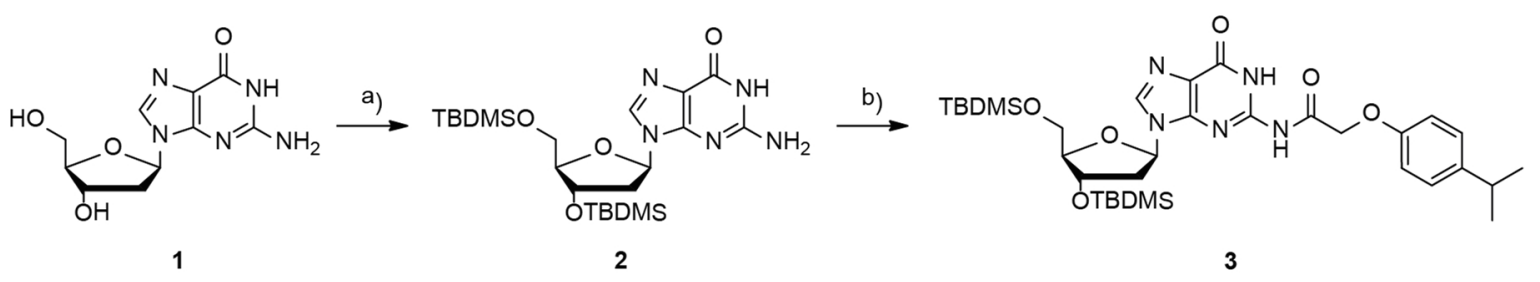

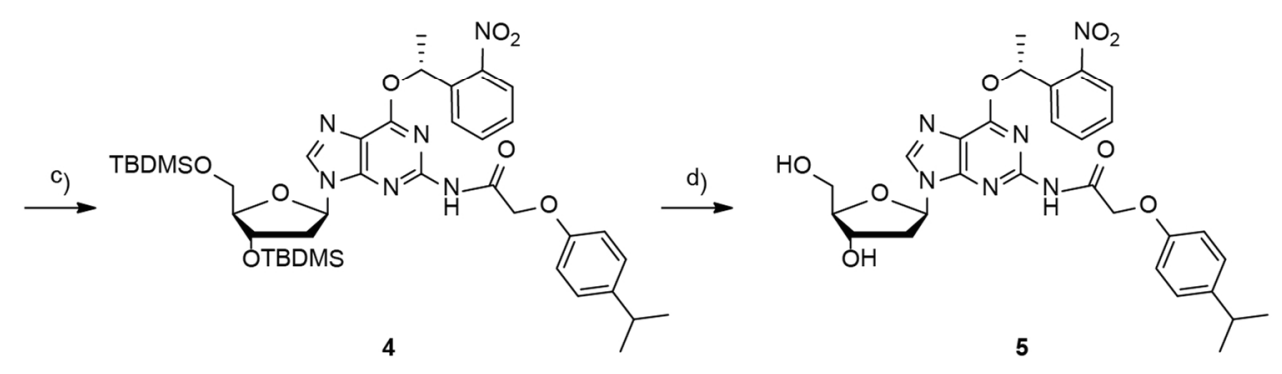<smiles>CC(C)c1ccc(OCC(=O)Nc2nc(O[C@@H](C)c3ccccc3[N+](=O)[O-])c3ncn([C@H](C)Oc4cccc(C(C)C)c4[N+](=O)[O-])c3n2)cc1</smiles>

Scheme 2: Overview of the synthesis of ${ }^{(R)-N P E} \mathrm{dG}$ phosphoramidite 7. a) TBDMS-Cl, imidazole, DMF, rt, quantitative; b) (4-isopropylphenoxy)acetyl chloride, pyridine, $0{ }^{\circ} \mathrm{C}$ to $\mathrm{rt}, 50 \%$; c) $(S)$-1-(2-nitrophenyl)ethanol (prepared according to the literature procedure(34)), $\mathrm{PPh}_{3}, \mathrm{DEAD}$ (40 wt\% in toluene), $\mathrm{THF}, 0^{\circ} \mathrm{C}$ to rt, $42 \%$; ) TBAF, AcOH, THF, rt, 93\%; e) 4,4'-dimethoxytrityl chloride, pyridine, $0^{\circ} \mathrm{C}$ to $\mathrm{rt}$, $91 \%$; f) 2-cyanoethyl- $N, N$-diisopropylchlorophosphoramidite, DIPEA, DCM, rt, 68\%.

\section{Synthesis of compound 2 (quantitative yield):}

$5.00 \mathrm{~g}$ 2'-deoxyguanosine 1 ( $18.7 \mathrm{mmol}, 1.0 \mathrm{eq}), 8.46 \mathrm{~g}$ tert-butyldimethylsilyl chloride ( $56.1 \mathrm{mmol}, 3.0 \mathrm{eq})$ and $8.92 \mathrm{~g}$ imidazole (130.8 mmol, $7.0 \mathrm{eq}$ ) were dissolved in $100 \mathrm{~mL}$ DMF and stirred at room temperature. After $1 \mathrm{~h}$, a white solid began to precipitate. The mixture was stirred overnight. The reaction was quenched by the addition of $150 \mathrm{~mL} E t \mathrm{H}$ and concentrated under reduced pressure. The residue was dissolved in $150 \mathrm{~mL}$ EtOAc and washed with dest. $\mathrm{H}_{2} \mathrm{O}, 1 \mathrm{M} \mathrm{HCl}$ and saturated aqueous $\mathrm{NaHCO}_{3}$ solution. The organic layer was dried over $\mathrm{Na}_{2} \mathrm{SO}_{4}$ and the solvent was removed under reduced pressure to give $\mathbf{2}$ as a white solid. The product was used for the following reaction without further purification.

\section{Synthesis of compound 3 (50\% yield):}

$3.50 \mathrm{~g}$ of the TBDMS protected nucleoside $2(7.1 \mathrm{mmol}, 1.0 \mathrm{eq})$ were dissolved in $25 \mathrm{~mL}$ pyridine. The solution was cooled to $0^{\circ} \mathrm{C}$ before $1.83 \mathrm{~mL}$ (4-isopropylphenoxy)acetyl chloride ( $10.6 \mathrm{mmol}, 1.5 \mathrm{eq}$ ) were added dropwise. The orange colored reaction mixture was stirred at $0{ }^{\circ} \mathrm{C}$ for $1 \mathrm{~h}$ and at room temperature for $4 \mathrm{~h}$. After quenching the reaction by the addition of $100 \mathrm{~mL} \mathrm{MeOH}$, the solvent was removed under reduced pressure. The crude product was purified by column chromatography $\left(\mathrm{SiO}_{2}, \mathrm{CH}_{2} \mathrm{Cl}_{2} / \mathrm{MeOH} 98: 2 \rightarrow 9: 1\right)$ to give 3 as a brownish solid.

\section{Synthesis of compound 4 (42\% yield):}

$2.00 \mathrm{~g}$ of 3 ( $3.0 \mathrm{mmol}, 1.0 \mathrm{eq}), 0.746 \mathrm{~g}(S)$-1-(2-nitrophenyl)ethanol (4.5 mmol, $1.5 \mathrm{eq})$ and $1.17 \mathrm{~g} \mathrm{PPh}_{3}(4.5 \mathrm{mmol}$, $1.5 \mathrm{eq}$ ) were dissolved in $35 \mathrm{~mL}$ THF. $0.7 \mathrm{~mL} \mathrm{DEAD}$ solution ( $40 \mathrm{wt} \%$ in toluene, $4.5 \mathrm{mmol}, 1.5 \mathrm{eq}$ ) were added dropwise. 


\section{Materials and Methods}

The reaction mixture was stirred at room temperature for $3 \mathrm{~d}$ and concentrated under reduced pressure. The residue was diluted with $200 \mathrm{~mL} \mathrm{CH} \mathrm{Cl}_{2}$ and washed with brine. The organic layer was dried over $\mathrm{Na}_{2} \mathrm{SO}_{4}$, the solvent removed under reduced pressure and the crude product purified by column chromatography $\left(\mathrm{SiO}_{2}\right.$, cyclohexane/EtOAc 95:5 $\rightarrow$ 3:1). Product 4 was isolated as a pale yellowish foam.

\section{Synthesis of compound 5 (93\% yield):}

$820 \mathrm{mg}$ of 4 ( $0.99 \mathrm{mmol}, 1.0 \mathrm{eq})$ were dissolved in $15 \mathrm{~mL}$ THF and $0.34 \mathrm{~mL}$ acetic acid $(6.0 \mathrm{mmol}, 6.0 \mathrm{eq}) \mathrm{and} 3.0 \mathrm{~mL}$ tetrabutylammonium fluoride solution ( $1 \mathrm{M}$ in THF, $3.0 \mathrm{mmol}$, $3.0 \mathrm{eq}$ ) were added. The reaction mixture was stirred at room temperature for $16 \mathrm{~h}$. The solvent was removed under reduced pressure and the crude product was purified by column chromatography $\left(\mathrm{SiO}_{2}, \mathrm{CH}_{2} \mathrm{Cl}_{2} \rightarrow \mathrm{CH}_{2} \mathrm{Cl}_{2} / \mathrm{MeOH}\right.$ 95:5) to give $\mathbf{5}$ as a yellowish foam.

\section{Synthesis of compound 6 ( $91 \%$ yield):}

$550 \mathrm{mg}$ of $\mathbf{5}$ ( $0.93 \mathrm{mmol}, 1.0 \mathrm{eq})$ were dissolved in $15 \mathrm{~mL}$ pyridine and cooled to $0{ }^{\circ} \mathrm{C} .378 \mathrm{mg} 4,4^{\prime}$-dimethoxytrityl chloride $(1.1 \mathrm{mmol}, 1.2 \mathrm{eq})$ were added slowly. The ice bath was removed and the yellow reaction mixture was stirred at room temperature for $3 \mathrm{~h}$. The solution was concentrated under reduced pressure and the residue diluted with $100 \mathrm{~mL} \mathrm{CH}_{2} \mathrm{Cl}_{2}$. The organic layer was washed with saturated aqueous $\mathrm{NaHCO}_{3}$ solution and dried over $\mathrm{Na}_{2} \mathrm{SO}_{4}$. The solvent was removed under reduced pressure. The crude product was purified by column chromatography $\left(\mathrm{SiO}_{2}, \mathrm{CH}_{2} \mathrm{Cl}_{2} / \mathrm{Et}_{3} \mathrm{~N}\right.$ 99:1 $\rightarrow \mathrm{CH}_{2} \mathrm{Cl}_{2} / \mathrm{MeOH}$ 95:5) to give 6 as a yellowish foam.

\section{Synthesis of compound 7 (68\% yield):}

To a solution of $753 \mathrm{mg}$ of $6(0.84 \mathrm{mmol}, 1.0 \mathrm{eq})$ and $733 \mu \mathrm{L}(\mathrm{Pr})_{2} \mathrm{NEt}(4.2 \mathrm{mmol}, 5.0 \mathrm{eq})$ dissolved in $15 \mathrm{~mL} \mathrm{CH}_{2} \mathrm{Cl}_{2}$ $376 \mu \mathrm{L}$ 2-cyanoethyl- $N, N$-diisopropylchlorophosphoramidite $(1.7 \mathrm{mmol}, 2.0 \mathrm{eq})$ were added. The reaction mixture was

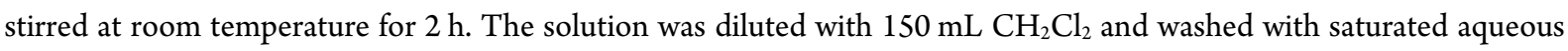
$\mathrm{NaHCO}_{3}$ solution. The organic layer was dried over $\mathrm{Na}_{2} \mathrm{SO}_{4}$ and the solvent removed under reduced pressure. The crude product was purified by column chromatography $\left(\mathrm{SiO}_{2}\right.$, hexane/EtOAc/Et ${ }_{3} \mathrm{~N}$ 95:4:1 $\rightarrow$ hexane/EtOAc $95: 5 \rightarrow 4: 1 \rightarrow 1: 1 \rightarrow 1: 2)$ to give 7 as a yellowish foam.

\section{Oligonucleotide synthesis:}

All photolabile modified oligonucleotides were synthesized on an ABI392 DNA/RNA synthesizer from Applied Biosystems. 0.3 M BTT from emp BIOTECH was used as activator. All oligonucleotides were synthesized and deprotected under UltraMILD conditions (Pac-dA-CE, iPrPac-dG-CE, Ac-dC-CE, dT-CE and dT SynBase ${ }^{\mathrm{TM}} 1000 \AA$ CPG purchased from Glen Research and Linktech) in $1 \mu \mathrm{mol}$ scales. $\mathrm{Pac}_{2} \mathrm{O}$ was used as capping reagent. short-2345 and wt-1245 (caged) were synthesized in DMTr-On while the remaining DNAs were synthesized in DMTr-Off mode. Deprotection was performed according to a protocol from Glen Research using $400 \mu \mathrm{L}$ concentrated ammonium hydroxide for $2 \mathrm{~h}$ at room temperature. After evaporation of the solvent at $4{ }^{\circ} \mathrm{C}$, the crude product was purified via RP-HPLC using an Agilent 1200 series instrument with an XBridge Peptide BEH C18 OBD Prep Column (300 А, $5 \mu \mathrm{m}, 10 \times 250 \mathrm{~mm}, 4.0 \mathrm{~mL} \mathrm{~min}^{-1}, 60^{\circ} \mathrm{C}$, gradient: 5-45.5\% MeOH in $400 \mathrm{mM}$ hexafluoro-2-propanol, $16.3 \mathrm{mM} \mathrm{NEt}_{3}$ buffer $\mathrm{pH}=8.2$ in $20 \mathrm{~min}$ ) from Waters. The solvent was evaporated at $4{ }^{\circ} \mathrm{C}$ using a vacuum concentrator (SpeedVac ${ }^{T M}$ from Thermo Fisher). The DMTr-group was cleaved by incubation of the DNA with $80 \%$ acetic acid for $20 \mathrm{~min}$ at room temperature. After evaporation at $4{ }^{\circ} \mathrm{C}$, the resulting DNA was again RP-HPLC purified using the same conditions as mentioned before. The solvent was removed using a vacuum concentrator. Finally, all samples were coevaporated several times with ultrapure water and lyophilized. 


\subsection{Spectroscopy}

All CD-spectra and CD-melting curves have been recorded on a JASCO spectropolarimeter J-810 at indicated temperatures. Spectra have been smoothed using a Savitzky-Golay filter. Time-resolved CD data were recorded on a Jasco J-810 spectropolarimeter in a $2 \mathrm{~mm}$ cuvette at $285 \mathrm{~K}$ using $10 \mu \mathrm{M}$ DNA in $5 \mathrm{mM} \mathrm{K}-\mathrm{P}_{\mathrm{i}}$-buffer (phosphate buffer: $38.5 \% \mathrm{KH}_{2} \mathrm{PO} 4+61.5 \% \mathrm{~K}_{2} \mathrm{HPO}_{4}$ ) at $\mathrm{pH}$ 7.0. Laser irradiation $(355 \mathrm{~nm}, 4 \mathrm{~W})$ within the cuvette was achieved via glass fiber connection to a laser set up (Paladin Advanced 355-8000) (Figure 31). Kinetics were recorded at $290 \mathrm{~nm}$ ( $1 \mathrm{~s}$ data interval) and in the range between $250-300 \mathrm{~nm}$ for control ( $6 \mathrm{~s}$ per spectrum resolution).

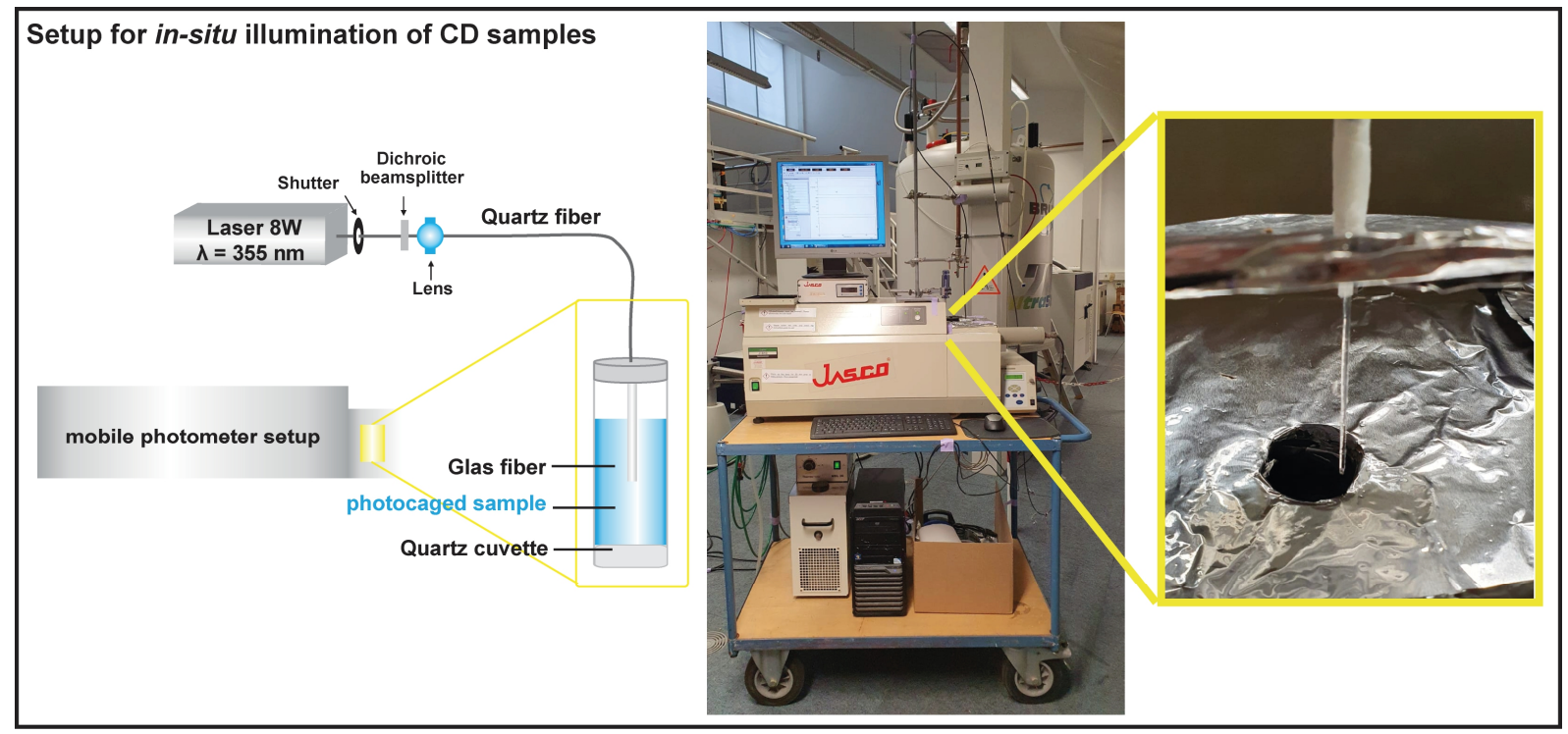

Figure 31: Setup for in-situ illumination of CD samples. Original setup design, idea and photos by J. Tassilo Grün.

\subsection{Non-Linear Regression and Global Fitting of Kinetic Data}

Kinetic traces have been processed in TopSpin 3.5pl7 (Bruker BioSpin) as either single signal intensity ("slice" - "extract column" or as sum-integral ("proj" - "sum") over a defined region. Traces then have been exported as .ascii-files and normalized for further analysis.

Non-linear regression of kinetic data was achieved with mono- or bi-exponential non-linear regression using the software SigmaPlot 12.5 (Systat Software Inc.).

For complex kinetic fits, numerical solutions for the system of differential equations were obtained from a python script (Appendix, commented script). The script was written by Dominik Brey, as intern under the supervision of me (the author of this thesis). The respective signal intensities of the NMRexperiments were normalized according to the mean equilibrium signal intensity. Global fit of the data was achieved with differential evolution using global optimization according to the respective kinetic model. Error estimation was estimated from a bootstrap method. Models were evaluated based on F-test analysis and residual plots. 


\subsection{NMR Spectroscopy}

All time-resolved NMR spectra were recorded on a Bruker AVIII HD $700 \mathrm{MHz}$ spectrometer equipped with a $5 \mathrm{~mm}$ z-axis TXI-HCP cryogenic probe at $298 \mathrm{~K}$ using $0.1 \mathrm{mM}$ DNA in $5 \mathrm{mM} \mathrm{K-P}$ (phosphate buffer: $38.5 \% \mathrm{KH}_{2} \mathrm{PO} 4+61.5 \% \mathrm{~K}_{2} \mathrm{HPO}_{4}$ ), $0.05 \mathrm{mM}$ DSS, $10 \% \mathrm{D} 2 \mathrm{O}$ at $\mathrm{pH} 7.0$ if not otherwise indicated. Water suppression was achieved using a jump-return-echo pulse scheme for timeresolved data or excitation sculpting for reference $1 \mathrm{D}$ spectra. For processing of data, Topspin $3.5 \mathrm{pl} 7$ (Bruker Biospin) was used. Time-resolved NMR experiments were performed as a pseudo-2D experiment. Laser irradiation within the NMR tube was triggered via a TLL connection to a laser set up (Paladin Advanced 355-8000) (Figure 32). Kinetics were recorded after $4 \mathrm{~s}$ of laser irradiation. Kinetic traces were subsequently averaged over 64 individual experiments. For $\mathrm{K}^{+}$-induced folding experiments, $0.1 \mathrm{mM}$ salt-free DNA in Bis-Tris-buffer at $\mathrm{pH}=7$ was used. $\mathrm{K}^{+}$-induced folding was achieved utilizing a rapid-mixing device, as described previously. ${ }^{144}$ Kinetic traces were subsequently averaged over 64 ( 8 for folding) individual scans. For light-induced folding experiments, kinetic traces were not averaged ( 1 scan per point). NOESY- and HMBC-spectra for assignment have been recorded using a jump-return-echo pulse scheme for water suppression. Spectrometers, Temperatures and sample conditions are indicated in the respective Figures.

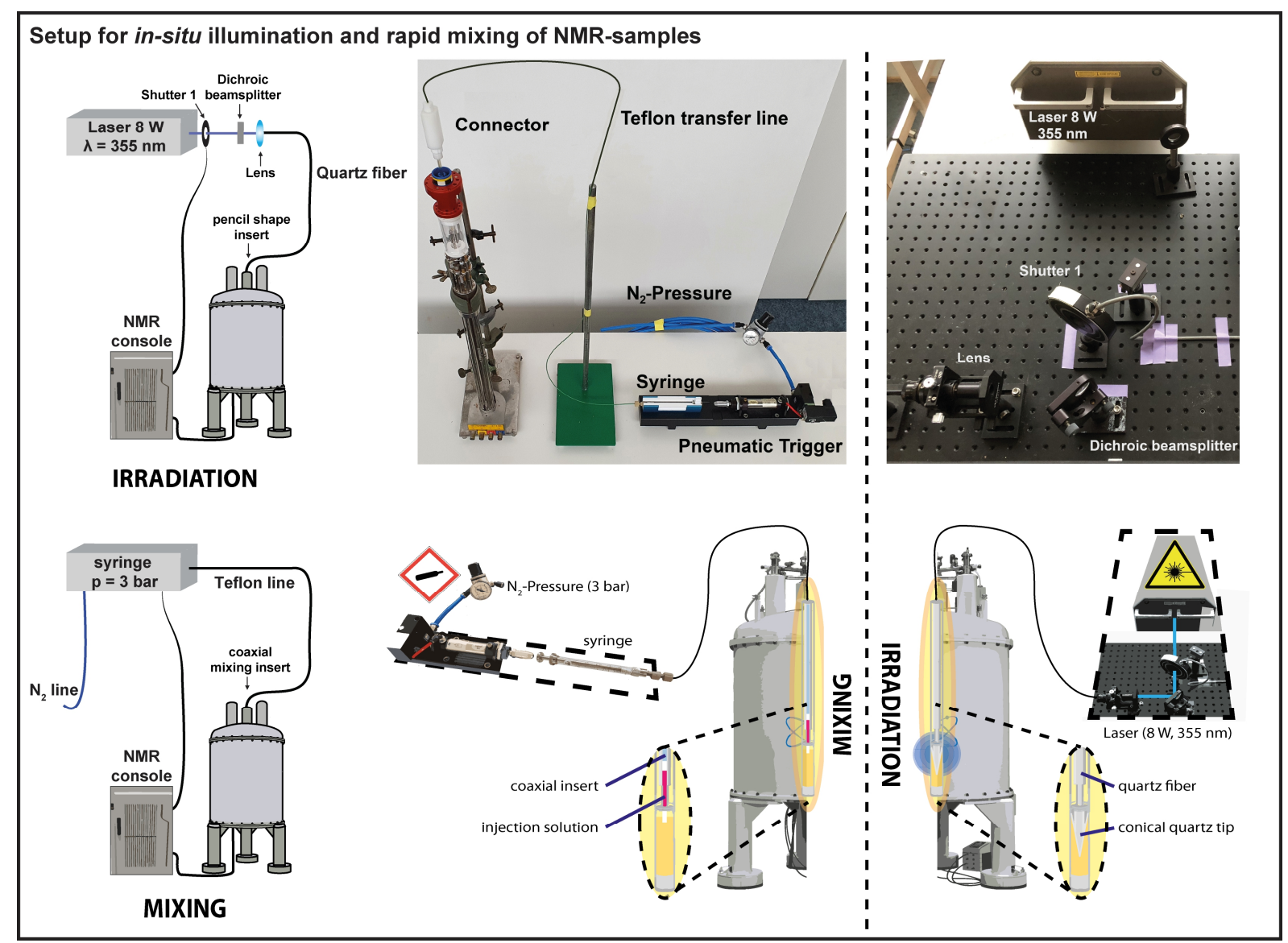

Figure 32: Setup for in situ illumination with a laser setup $(8 \mathrm{~W}, 355 \mathrm{~nm})$ connected via a glass fiber to a Shigemi tube with a conical quartz tip. ${ }^{696}$ Setup for in situ rapid mixing with a mixing device introduced by Hore et al. ${ }^{683}$. Photos from the setups are originally taken from J. Tassilo Grün. 
Materials and Methods 


\section{G-Register Exchange Dynamics}

\subsection{Overview}

This chapter examines the kinetics of folding from unfolded states and refolding from trapped, isolated folded states back to their native equilibrium for the major G4 conformation (2345) found in the cMYC promoter (2.4.2). The 18-mer oligonucleotide $c M Y C$ 2345-X3 (3.1.1) can coexist in two different G-register isomers (Figure 33 (left); and chapters 2.1.3, 2.4.3). The G-register exchange dynamics for the interconversion of the two parallel $c M Y C$ G4 conformations have been compared to the interconversion of two conformations from the hTERT G4 that co-exists in a hybrid and a parallel conformation. The experimental data focus on the dynamics associated to the refolding between either of the two-state systems.

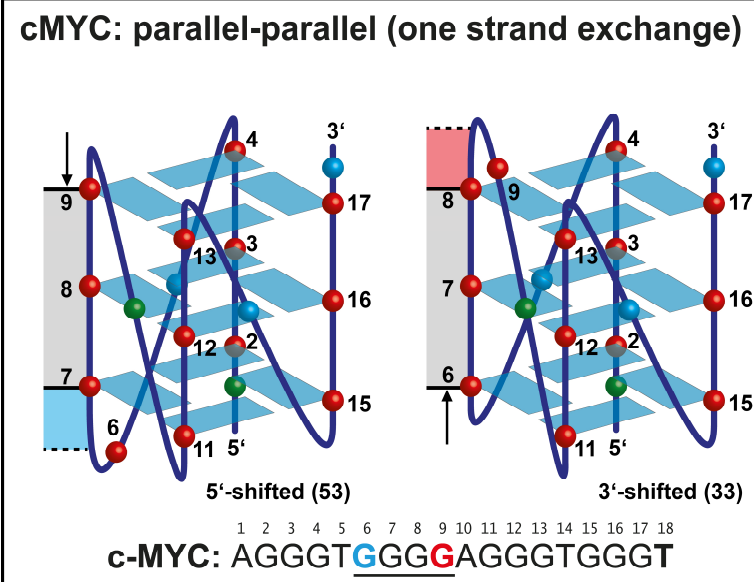

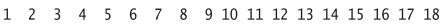
c-MYC: AGGGTGGGGAGGGTGGGT

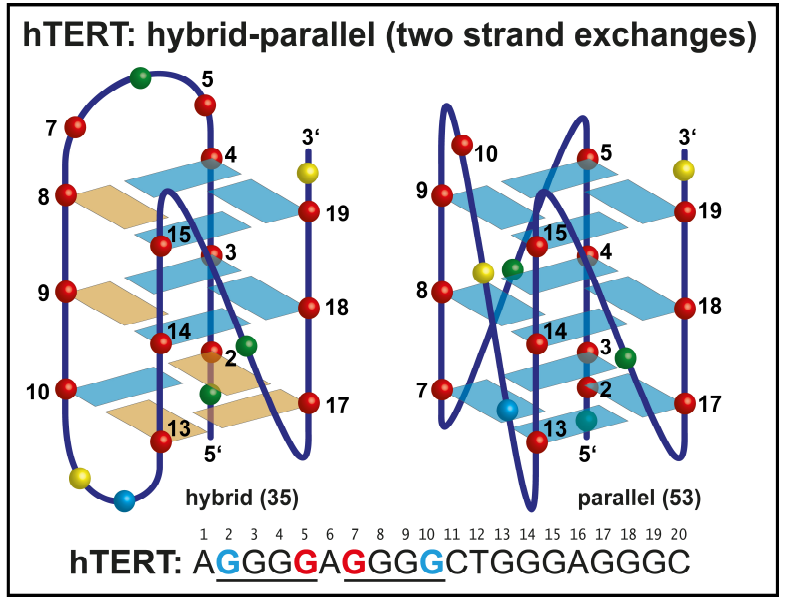

Figure 33: Schematic representation for two different pairs of G-register shift related G4 conformations. (left) Two parallel cMYC G-register isomers $(53,33)$ with G-tract III shifted either towards the 5'-terminal tetrad (5) or the 3'-terminal tetrad (3). G-tract V can form two additional isomeric states (3'- and 5'-shifted) in the wildtype sequence, but due to a G-to-T modification on residue 18 the 3 '-shifted state is locked. (right) Two G-register isomers from an $h T E R T$ G4 in hybrid (35) or parallel (53) conformation.

The main results presented here have been published in Grün et al. 2020. ${ }^{732}$ Experiments in chapter 4.6.1 have been conducted by Christopher Hennecker and Robert W. Harkness in the group of Anthony K. Mittermaier within a joint collaboration. All shown figures have been created originally by me (the author of this thesis) and are presented modified or unmodified as compared to the published version: Reprinted (adapted) with permission from J. Am. Chem. Soc. 2020, 142, 1, 264-273. Copyright 2020 American Chemical Society. 


\section{2 $\mathrm{K}^{+}$-Induced Folding into G-register Isomers}

Extensive desalting (3.1) of the native 18-mer $c M Y C 2345$-X3 oligonucleotides yields ${ }^{1} \mathrm{H}$-NMR spectra that show no imino proton signals in the ppm-range typical for Hoogsteen base pair interactions in $\mathrm{K}^{+}$-bound G-quadruplexes (Figure 34). This state has been considered unfolded after the careful analysis of the NMR-spectra, even though the formation of pre-folded states cannot completely be precluded. After addition of $\mathrm{K}^{+}$to a final concentration of $5 \mathrm{mM}$, the native folded ensemble can be recaptured. In thermodynamic equilibrium the folded state of $c M Y C 2345-\mathrm{X} 3$ is consisting of two coexisting G-quadruplex conformations, as will be shown and discussed further in this chapter.

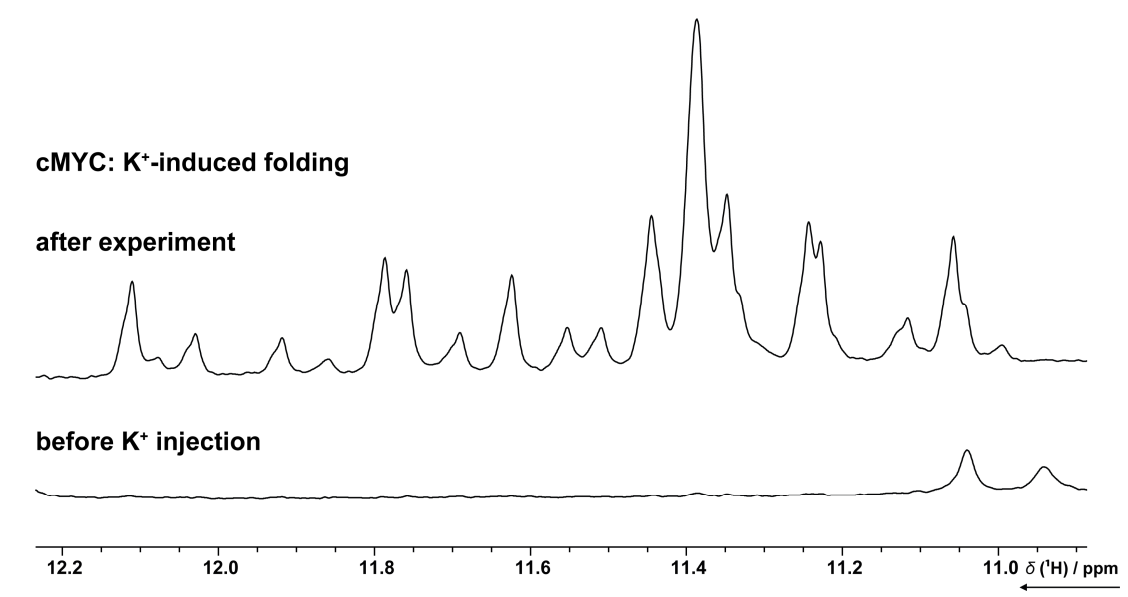

Figure 34: ${ }^{1} \mathrm{H}-\mathrm{NMR}$ spectra of $c M Y C 2345-\mathrm{X} 3$ in $\mathrm{K}^{+}$-free (lower) Bis-Tris buffered solution and several hours after addition of $5 \mathrm{mM} \mathrm{KCl}$ (upper). Comparably broad signals in the downfield $(>12.1 \mathrm{ppm})$ and upfield $(<11.1 \mathrm{ppm})$ shifted region indicate some pre-associated state that are unrelated to the $\mathrm{K}^{+}$-bound state. (0.1 mMDNA, $5 \mathrm{mM}$ Bis-Tris buffer $(\mathrm{pH}=7.0)$, 90/10: $\mathrm{H}_{2} \mathrm{O} / \mathrm{D}_{2} \mathrm{O}, 700 \mathrm{MHz}, 298 \mathrm{~K}$, jr-echo water suppression)

The folding reaction was initiated by a rapid injection of $\mathrm{K}^{+}$using the rapid-mixing device outlined in section 2.5.3 and 3.4. Time correlated single scan 1D spectra were recorded as pseudo-2D dataset. Kinetic traces for the folding reaction were then extracted by plotting the respective signal intensities as function of time. The spectral resolution $(\sim 1.16 \mathrm{~s})$ of the imino proton signals allows tracking of the kinetics of individual signals that represent either of the two folded conformations (Figure 35).

The folding kinetics exhibit a clear biphasic behaviour: (i) rapid folding into the two individual states and (ii) slow reequilibration to thermodynamic distribution. The kinetics also reveal that the minor conformation is slightly kinetically favoured and overshoots in the first folding phase, compared to thermodynamic equilibrium. The kinetics were analysed both with bi-exponential fitting and with global fitting to a kinetic model (Table 4).

The easiest model to describe the folding mechanism is a direct refolding from the minor to the major state (Figure 35). The global fitting procedure (3.3) yields numerical solutions for the individual rate constants. 
a)

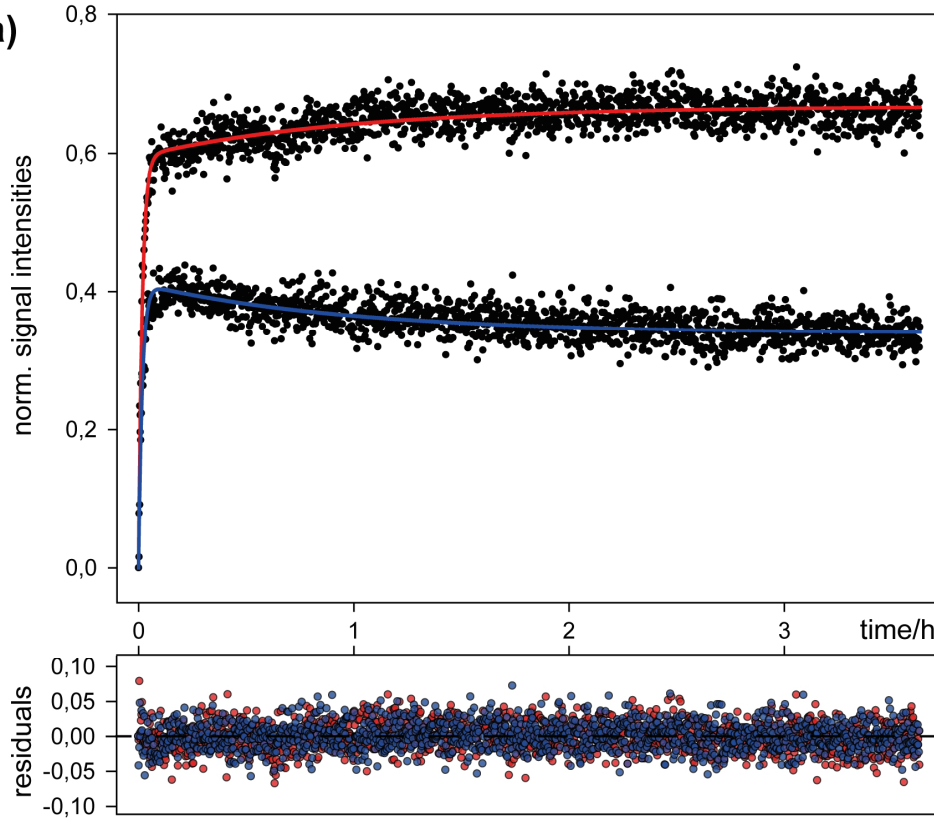

b)

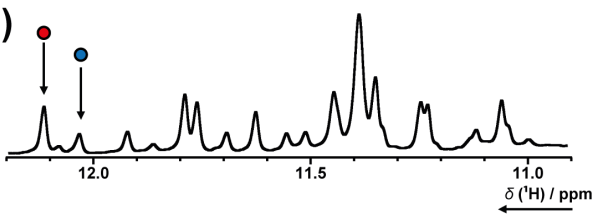

c)

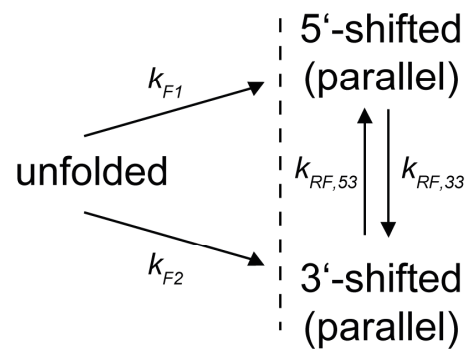

Figure 35: Folding transition of $c M Y C$ 2345-X3 G-register isomers. (a) Kinetic traces of individual imino proton signals (b), representative for the two folded conformations, shown in red (cMYC-2345-33) and blue (cMYC-2345-53). Fitting curves and residuals result from global fitting to the depicted kinetic model (c).

The residual plot shown in Figure 35 a results from fitting to the depicted kinetic model (Figure $35 \mathrm{c}$ ). Errors for bi-exponential fitting $\left(\mathrm{k}_{\mathrm{obs}}\right)$ are within $5 \%$, but should be considered as estimates.

Table 4: Kinetic rate constants for the overall (re-)folding transitions $\left(\mathrm{k}_{\mathrm{obs}}\right)$ derived from exponential fitting using nonlinear regression and results from global fitting to the kinetic model depicted in Figure 35. For non-linear regression, the calculated standard error is within $5 \%$ as well as the error estimate from fitting residuals.

\begin{tabular}{|c|c|c|c|c|c|}
\hline \multicolumn{3}{|c|}{$k_{o b s}\left[h^{-1}\right]$} & Conformation & folding rate $\left[h^{-1}\right]$ & refolding rate $\left[h^{-1}\right]$ \\
\hline \multirow{2}{*}{$c M Y C$} & Folding & Refolding & $2345-53$ & $\mathrm{k}_{\mathrm{F}, 53}=35$ & $\mathrm{k}_{\mathrm{RF}, 53}=0.35$ \\
\hline & 59 & 1.0 & $2345-33$ & $\mathrm{k}_{\mathrm{F}, 33}=25$ & $\mathrm{k}_{\mathrm{RF}, 33}=0.69$ \\
\hline
\end{tabular}

A Bis-Tris buffer was used instead of a phosphate buffer to obtain the $\mathrm{K}^{+}$-free state, which is different from NMR spectra of most other shown samples. The spectra indicate no structural changes in different buffers. Further, no influence of phosphate vs. Bis-Tris buffer on folding kinetics was observed in control experiments. 


\subsection{Light-Induced Refolding of Caged G-Register Isomers}

\subsubsection{Conformational Selection of G-Register Isomers}

The second, slow phase of the folding reaction indicates that refolding occurs, which gives a dynamic link between the two folded conformations. The expected timescale for this rearrangement is to slow for NMR methods in equilibrium to investigate, if the discussed direct refolding mechanism is correct. The folding trajectory however, could also be affected by underlying on- or off-pathway intermediates or pre- and misfolded structures that cause the respective kinetic behaviour. In this regard, the corresponding population change would less be a refolding reaction from stable folded structures, but more a feature of perturbed, retarded folding from this somewhat partially folded states. The refolding dynamics therefore should be separated from initial folding and investigated independently.

The $c M Y C$ 2345-X3 oligonucleotide was synthesized in a way to carry a (R)-NPE photolabile protecting group either at position G6 to yield $c M Y C 2345-53$ or at position G9 to yield $c M Y C 2345-33$ (Figure 36). The ${ }^{1} \mathrm{H}-\mathrm{NMR}$ spectra show that this approach is suitable to select either one of the two conformations, as the native spectrum directly decomposes into the spectra of the individual, trapped single conformations.
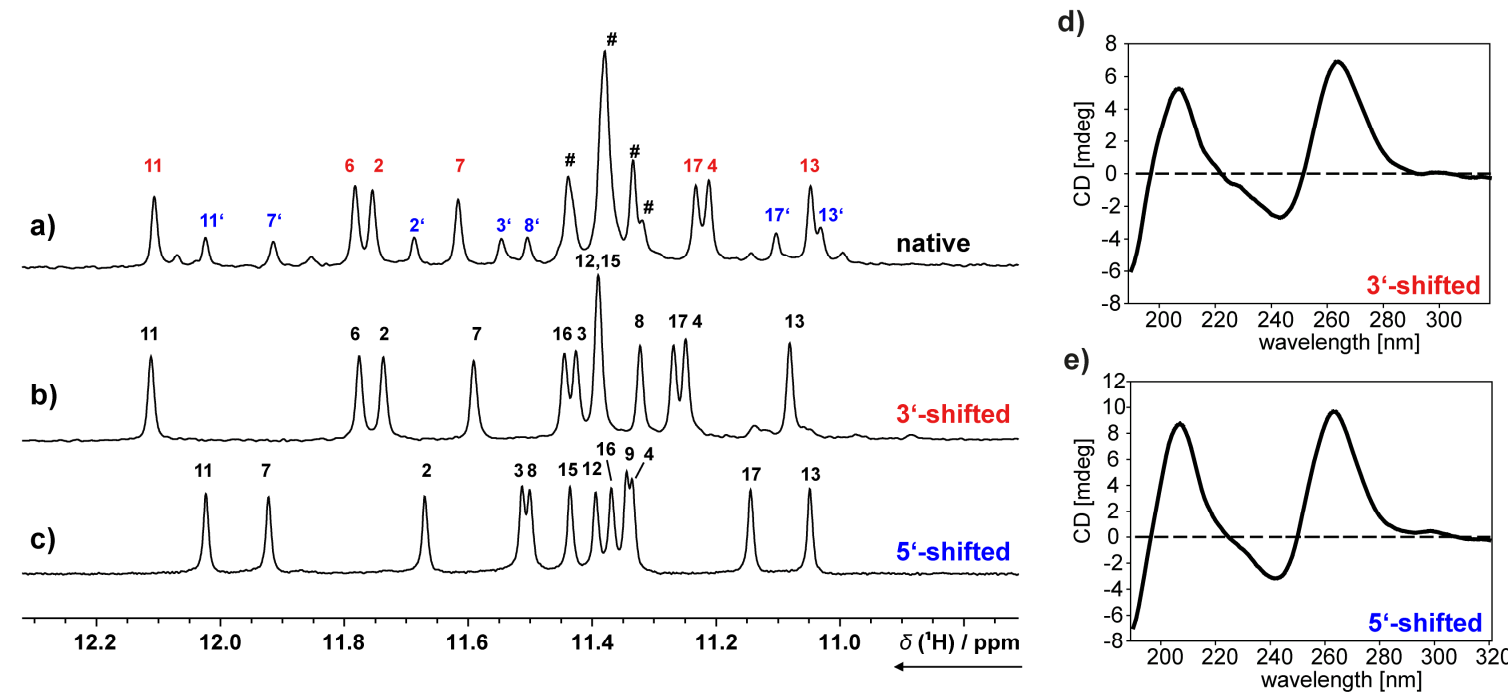

e)

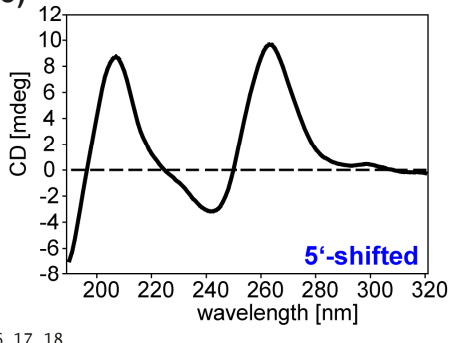

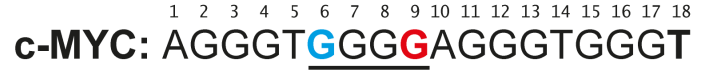

Figure 36: (a-c) 1D- ${ }^{1} \mathrm{H}-\mathrm{NMR}$ spectra of a) native cMYC 2345-X3, b) trapped (caged) 3'-shifted conformation (red) and c) caged 5'-shifted conformation (blue). (0.1 mM DNA, 5 mM K- $\mathrm{P}_{\mathrm{i}}$ buffer ( $\left.\mathrm{pH}=7.0\right), 90 / 10: \mathrm{H}_{2} \mathrm{O} / \mathrm{D}_{2} \mathrm{O}, 700 \mathrm{MHz}, 298 \mathrm{~K}$, jr-echo water suppression) (d-e) CD-spectra of caged 3'-shifted (d) and 5'-shifted (e) conformations. The spectra show characteristic signatures for all-parallel G4 conformations. (10 $\mu \mathrm{M}$ DNA, $5 \mathrm{mM} \mathrm{K}-\mathrm{P}_{\mathrm{i}}$ buffer $\left.(\mathrm{pH}=7.0), 298 \mathrm{~K}\right)$

CD spectra of the two conformations show that the expected parallel conformation is maintained (Figure 36 d, 3'-shifted 33 and e, 5'-shifted 53). Spectral assignment (Figure 37, Table 5) of the respective conformations was achieved via NOESY (Figure 38, Figure 39) interactions, as well as longrange $\mathrm{HMBC}$ correlations and H-D-exchange experiments (Appendix 6.1, Figure 66). Taken together the expected overall all-parallel folding topology can be certainly supposed. 

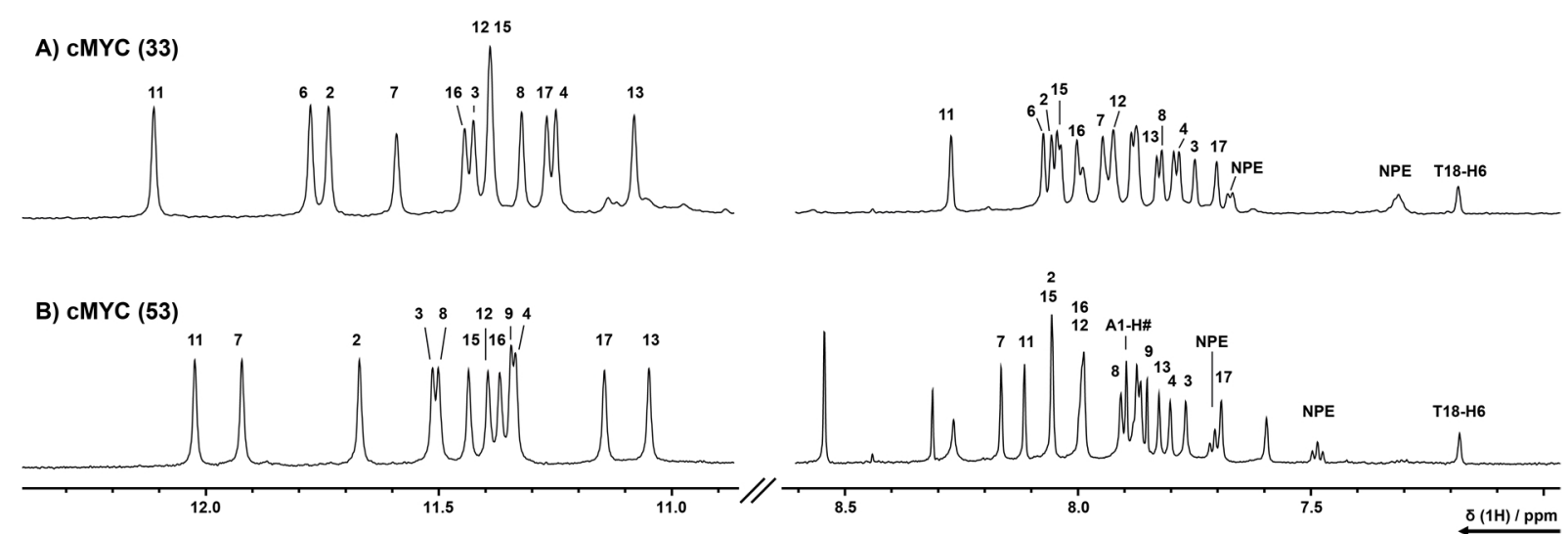

Figure 37: Assigned $1 \mathrm{D}^{1} \mathrm{H}-\mathrm{NMR}$ spectra showing the imino and aromatic proton regions of photocaged A) $c M Y C-18$ (33) and B) cMYC-18 (53) G-quadruplex, (700 MHz, $298 \mathrm{~K})$. Referenced to DSS.

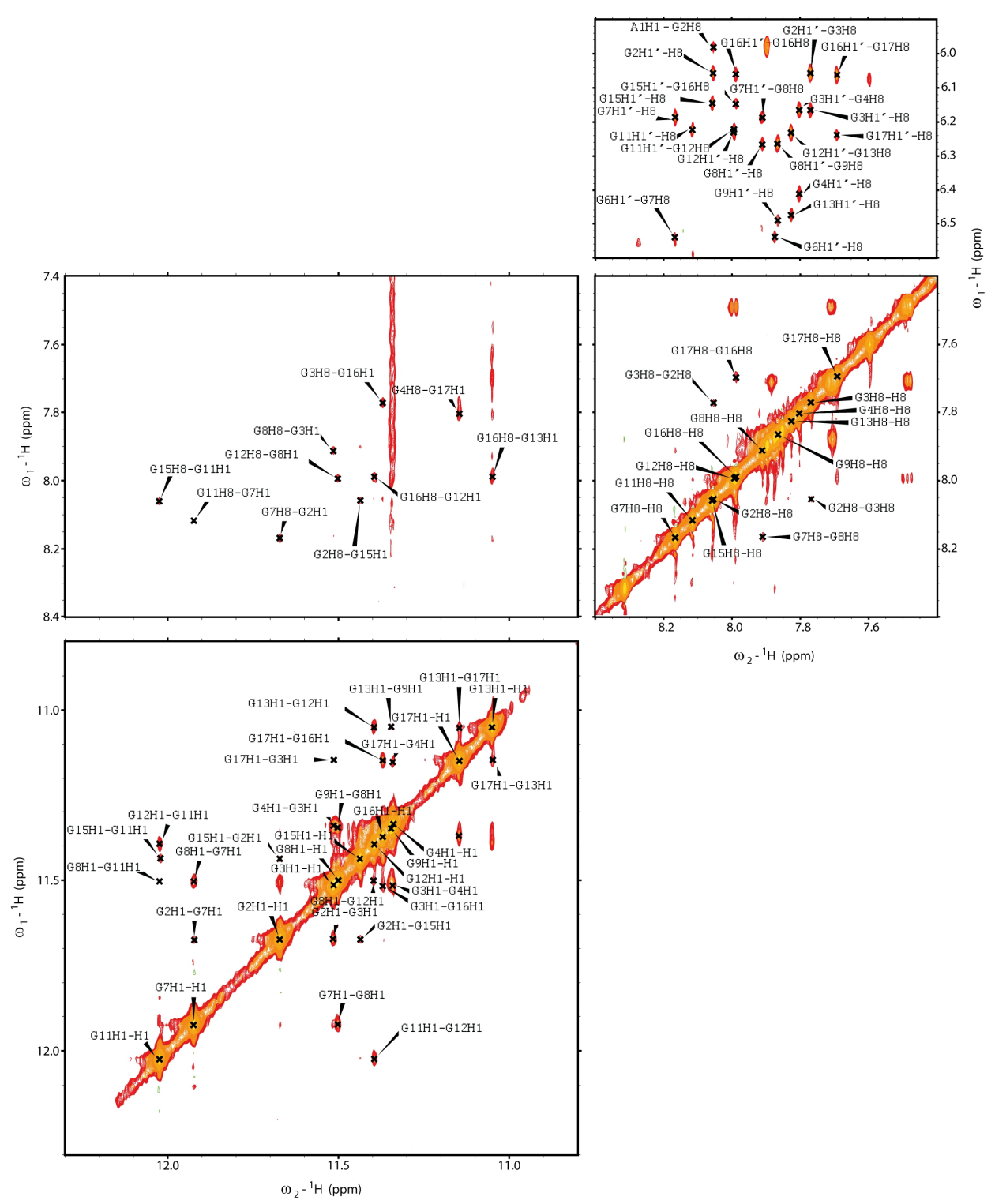

Figure 38: Assigned 2D-NOESY-spectra with characteristic H1-H1, H1-H8, H8-H8 and H1'-H8 regions of the photocaged 5'-shifted cMYC-2345-53 G-quadruplex. Recorded with $8192 \times 1024$ points ( $800 \mathrm{MHz}, 298 \mathrm{~K})$ using a pulse-sequence with jump-return-echo water-suppression. (5 mM K-P $\mathrm{P}_{\mathrm{i}}$ buffer $\left.(\mathrm{pH}=7.0), 298 \mathrm{~K}\right)$ 

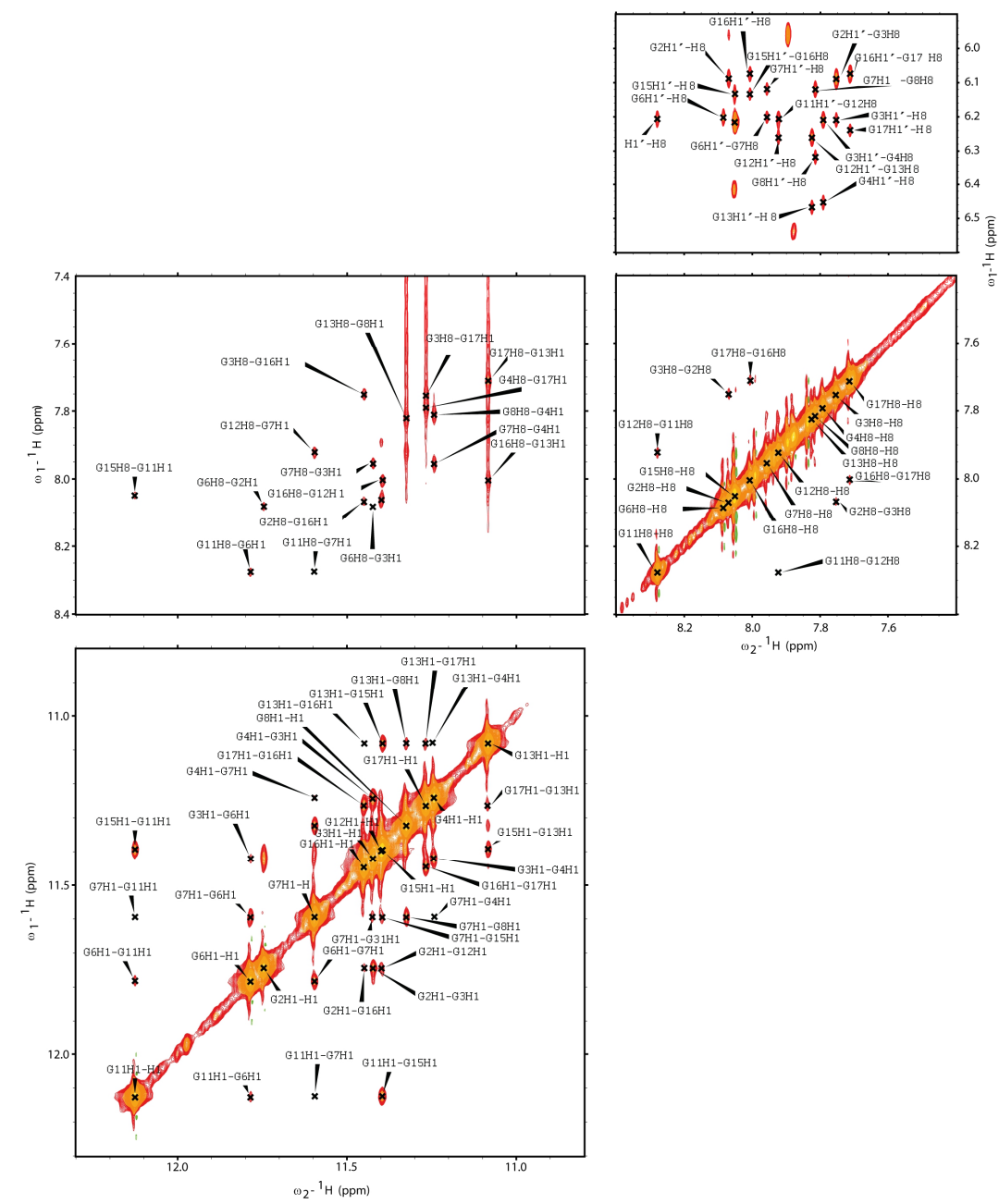

Figure 39: Assigned 2D-NOESY-spectra with characteristic H1-H1, H1-H8, H8-H8 and H1'-H8 regions of the photocaged 3'-shifted $c M Y C$-2345-33 G-quadruplex. Recorded with $8192 \times 1024$ points $(600 \mathrm{MHz}, 298 \mathrm{~K})$ using a pulse-sequence with jump-return-echo water-suppression. (5 mM K- $\mathrm{P}_{\mathrm{i}}$ buffer $\left.(\mathrm{pH}=7.0), 298 \mathrm{~K}\right)$

Table 5: Chemical shifts of assigned $\mathrm{H} 1$ imino and $\mathrm{H} 8$ aromatic proton signals of photocaged $c M Y C$ conformations at $298 \mathrm{~K}$ in $5 \mathrm{mM} \mathrm{K}^{+}(\mathrm{pH}=7.0)$. Referenced to DSS.

\begin{tabular}{c|c|c|c|c}
\multicolumn{2}{c}{} & \multicolumn{2}{c}{$c \mathbf{M Y C}(\mathbf{5 3})[\mathbf{p p m}]$} & \multicolumn{2}{c}{$\boldsymbol{c M Y C}(\mathbf{3 3})[\mathbf{p p m}]$} \\
Residue & $H 1$ & $H 8$ & $H 1$ & $H 8$ \\
\hline G2 & 11.67 & 8.05 & 11.75 & 8.07 \\
G3 & 11.52 & 7.77 & 11.42 & 7.75 \\
G4 & 11.34 & 7.80 & 11.24 & 7.79 \\
G6 & - & - & 11.78 & 8.09 \\
G7 & 11.92 & 8.17 & 11.60 & 7.96 \\
G8 & 11.50 & 7.91 & 11.32 & 7.82 \\
G9 & 11.35 & 7.87 & - & - \\
G11 & 12.02 & 8.12 & 12.13 & 8.28 \\
G12 & 11.40 & 8.00 & 11.40 & 7.92 \\
G13 & 11.05 & 7.83 & 11.08 & 7.83 \\
G15 & 11.44 & 8.06 & 11.40 & 8.05 \\
G16 & 11.37 & 7.99 & 11.45 & 8.00 \\
G17 & 11.15 & 7.69 & 11.27 & 7.71
\end{tabular}




\subsubsection{Refolding into a Two-State G-Register Ensemble}

The refolding reaction was initiated by photolysis using the laser setup outlined in section 3.4. Time correlated single scan 1D spectra were recorded as pseudo-2D dataset and afterwards summed up to yield 64 scans per point in time. Kinetic traces for the refolding reaction were then extracted by plotting the respective signal intensities as function of time (Figure 40).

a)

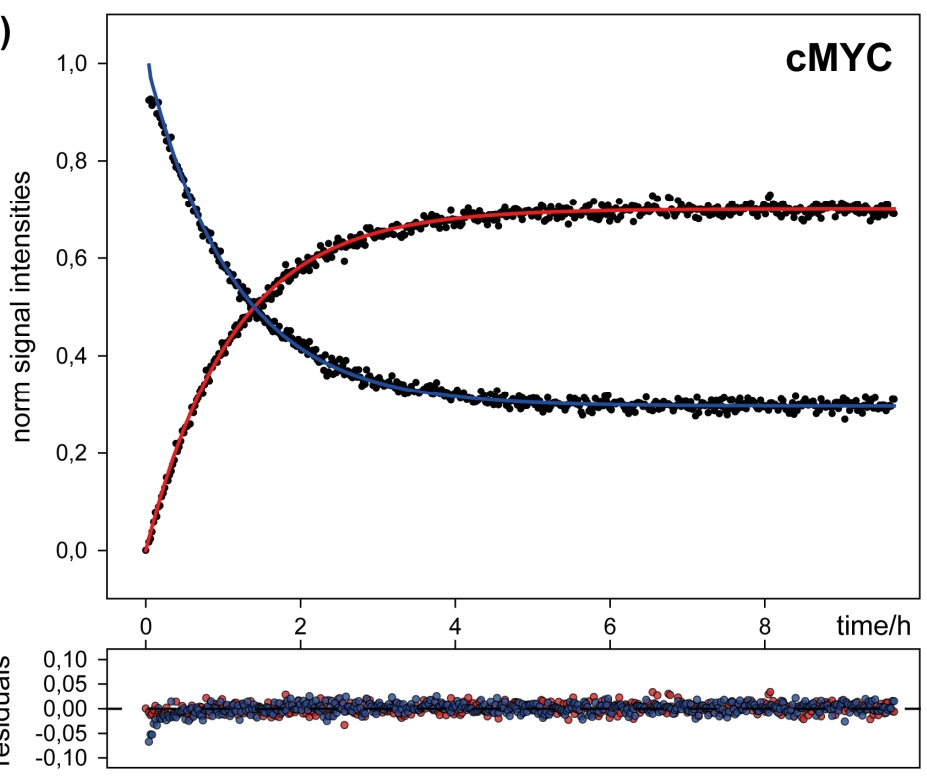

b)

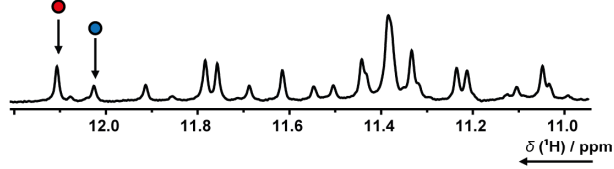

5'-shifted (parallel)

c)

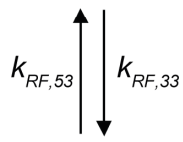

3'-shifted

(parallel)

Figure 40: Refolding transition of $c M Y C$ 2345-X3 G-register isomers. (a) Kinetic traces of individual imino proton signals (b), representative for the two folded conformations, shown in red (cMYC-2345-33) and blue (cMYC-2345-53). Fitting curves and residuals result from global fitting to the depicted kinetic model (c).

The kinetic data reveal that the refolding process is slow, taking place for several hours at room temperature. The signals for the initially $100 \%$ populated single conformation decay, while signals for the second conformation build-up. These kinetics have been fitted globally according to a two-state kinetic model (Figure $40 \mathrm{c}$ ), yielding the rate constants $k_{R F, 53}$ and $k_{R F, 33}$ shown in Table 6 . The observable rate constant $\mathrm{k}_{\mathrm{obs}}$ for the refolding process was derived from mono-exponential fitting. The rates are in good agreement if $\boldsymbol{k}_{o b s}=\boldsymbol{k}_{R F, 53}+\boldsymbol{k}_{R F, 33}$ is assumed $\left(0.85 \mathrm{~h}^{-1} \approx 0.9 \mathrm{~h}^{-1}\right)$.

Table 6: Kinetic rate constants for the overall refolding transition $\left(\mathrm{k}_{\mathrm{obs}}\right)$ derived from exponential fitting using non-linear regression and results from global fitting to the kinetic model depicted in Figure 40. For non-linear regression, the calculated standard error is within $5 \%$ as well as the error estimate from fitting residuals.

\begin{tabular}{c|cc}
\multicolumn{2}{c}{$k_{o b s}\left[h^{-1}\right]$} & \multicolumn{1}{c}{ refolding rate $\left[h^{-1}\right]$} \\
\hline \multirow{2}{*}{$c M Y C$} & Refolding & $\mathrm{k}_{\mathrm{RF}, 53}=0.27$ \\
& 0.85 & $\mathrm{k}_{\mathrm{RF}, 33}=0.63$
\end{tabular}

The two-state model is the simplest model that can be applied to this system of two conformations. Neither long-lived intermediate conformations can be detected, nor does the correlation between both traces indicate an accumulation of unfolded species. 
The total signal intensity remains constant during the progression of the experiment. However, the overall kinetics are slow. The existence of any additional short-lived intermediate states (potentially unfolded states) that indicate more complex refolding cannot be postulated or precluded based on the experiments.

\subsubsection{Refolding into a Full Set G-Register Ensemble}

The refolding reported in 4.3.2, encompasses the two majorly populated $c M Y C$ G-register isomers 2345-53 and 2345-33. To investigate the kinetic effect of the full conformational space of the 18-mer cMYC-2345 wt-sequence, a trapped conformation was prepared using a second photocage at the 3'-terminal G-residue (G18). This position is replaced with a G-to-T mutation in all other presented experiments. Potentially, the incorporation of G18 into tetrad formation can yield two additional G-register isomers, resulting from a strand shift in the 3-terminal G-tract (namely the 35 and 55 conformation).

The incorporation of the second photocage was successful to trap the 53-conformation in a single folded state without further distortions to the structure (Figure $41 \mathrm{~b}$ ). The refolding kinetics have been monitored after photolysis and analysed with mono-exponential fitting. The observable rate constant for refolding was obtained as a function of the build-up of the $c M Y C-33$ conformation (Figure $41 \mathrm{c}$ ). The refolding $\left(0.54 \mathrm{~h}^{-1}\right)$ is $\sim 36 \%$ slower compared to the two state refolding process between the 53 and 33 conformations $\left(0.85 \mathrm{~h}^{-1}\right)$. This shows that the refolding process in the $c M Y C$ G4 forming sequence that is able to adopt all four possible G-register isomers is clearly more complex. The NMR spectrum after the refolding process shows additional imino proton signals that indicate a polymorphic ensemble with more than two populated conformations (Figure 41 a).

The enlarged conformational space during refolding enables additional interactions in the transitory ensemble leading to kinetic traps that decelerate the process. These additional conformational states can only occur if the 3'-terminal G-tract (G-tract V) unfolds from the G4-core.

a)

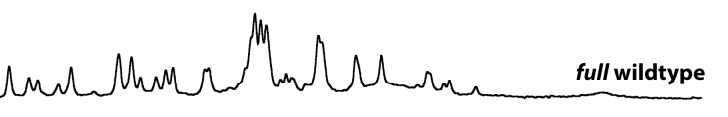

b)

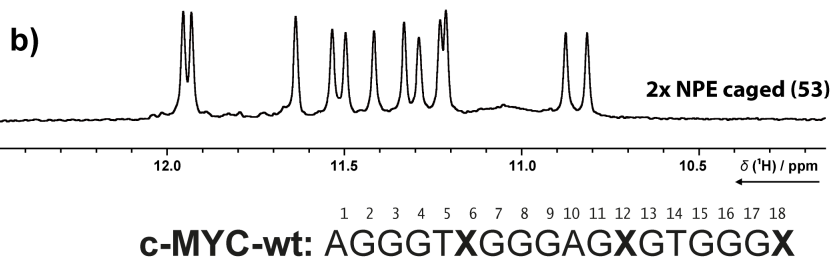

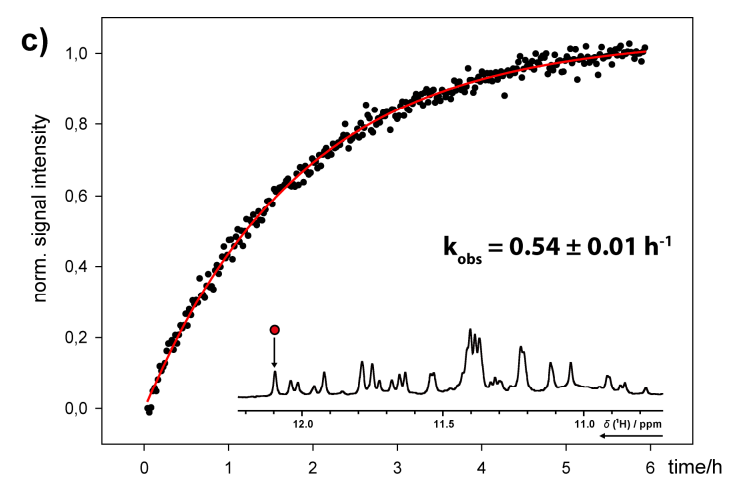

Figure 41: ${ }^{1} \mathrm{H}$ NMR spectrum of a) the 18-mer $c M Y C$ wt-sequence with all four possible G-register isomers in a native oligonucleotide; and b) with two $(R)$-NPE cages at position G6 and G18 (marked with X in the sequence, conformation53). c) Kinetic trace for light-induced refolding and mono-exponential fitting (red) to yield $\mathrm{k}_{\mathrm{obs}}$. 


\subsection{G-Register Polymorphism in the hTERT Promoter G-Quadruplex}

The human TElomerase Reverse-Transcriptase ( $h T E R T)$ gene promoter region possesses a G4 forming element that has multiple G-rich strands, able to fold into a manifold of different G4 conformations. ${ }^{102,103}$ Two co-existing conformations have been published for a $20 \mathrm{nt}$ long sequence that can be found $60 \mathrm{nt}$ upstream of the TSS.

These conformations feature G-register isomerism as they incorporate different G-residues into the G4 tetrads. However, the reported conformations are markedly different, since they adopt either an all-parallel or a hybrid conformation. To investigate the G-register exchange dynamics in comparison to the reported kinetics of the $c M Y C$ G-register isomers, the same photocaging strategy was applied. For each oligonucleotide, two photocages have been incorporated to isolate a specific conformation, because the hTERT conformations distinguish for G-register shifts in G-tract I and II. The photocaging strategy was successful to trap and isolate the parallel and the hybrid conformations from the wtsequence (Figure 42). Some minor additional imino proton signals are visible that vanish immediately after uncaging. This set of signals might arise from different stacked photocage orientations.

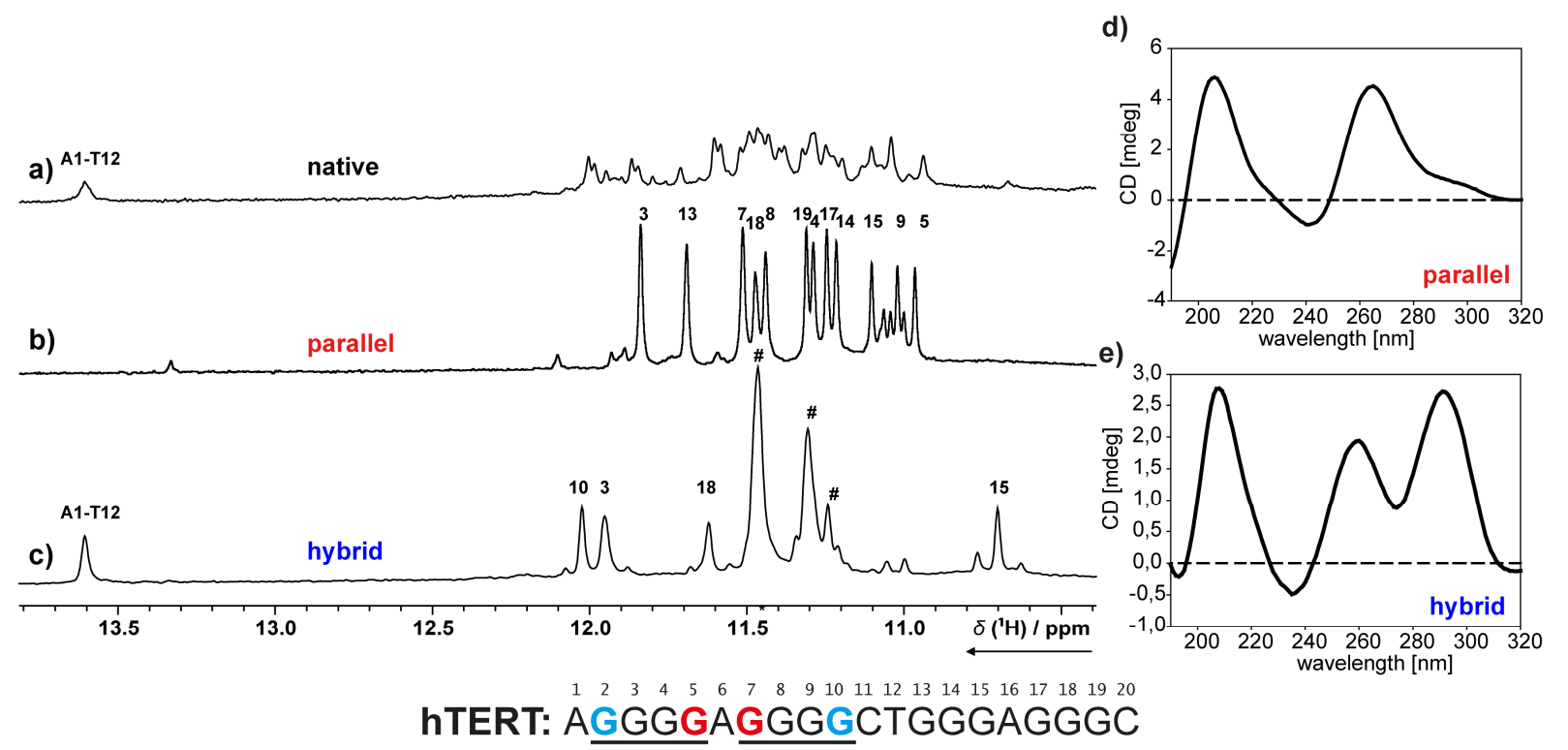

Figure 42: (a-c) 1D- ${ }^{1} \mathrm{H}-\mathrm{NMR}$ spectra of a) native $\left.h T E R T, \mathbf{b}\right)$ trapped (caged) parallel conformation (red) and c) caged hybrid conformation (blue). (0.1 mM DNA, 5 mM K- $\mathrm{P}_{\mathrm{i}}$ buffer $\left(\mathrm{pH}=7.0\right.$ ), 90/10: $\mathrm{H}_{2} \mathrm{O} / \mathrm{D}_{2} \mathrm{O}, 700 \mathrm{MHz}, 298 \mathrm{~K}$, jr-echo water suppression) (d-e) CD-spectra of caged parallel (d) and hybrid (e) conformations. The spectra show characteristic signatures for all-parallel and hybrid G4 conformations. Assignment taken from Lim et al. ${ }^{102}$, \#-signals have not been assigned due to spectral overlap. (10 $\mu$ M DNA, 5 mM K-P $\mathrm{P}_{\mathrm{i}}$ buffer $\left.(\mathrm{pH}=7.0), 298 \mathrm{~K}\right)$

The refolding kinetics have been measured and analyzed as described in 4.3.2. Figure 43 shows representative kinetic traces, starting from a caged parallel conformation. Fitted in blue is the decay of the parallel conformation, fitted in red the build-up of the hybrid conformation. The downfield-shifted signal ( $13.6 \mathrm{ppm})$ can be assigned to an A1-T12 Watson-Crick base pair, which serves as a specific probe for the hybrid conformation. 
The refolding reaction is slow with an observable rate constant (Table 7) that is comparable to the refolding of the $c M Y C$ G-register isomers.

This observation was unexpected; as the initial hypothesis was that the refolding should be slower, due to a drastically greater degree of unfolding needed for interconversion of a hybrid to a parallel conformation. The postulation of an unfolded ensemble in the kinetic model (Figure $43 \mathrm{c}$ ) is based both on analysis of fitting statistics and rational considerations, because refolding between the two states necessarily requires unfolding to a significant extent. The residual plot shows $\sim 5 \%$ systematic deviations for the fitting in the initial $2 \mathrm{~h}$ of the experiment, which gives indications for a more complex underlying mechanism. The kinetic model is however adequate to describe the overall refolding progression.

a)

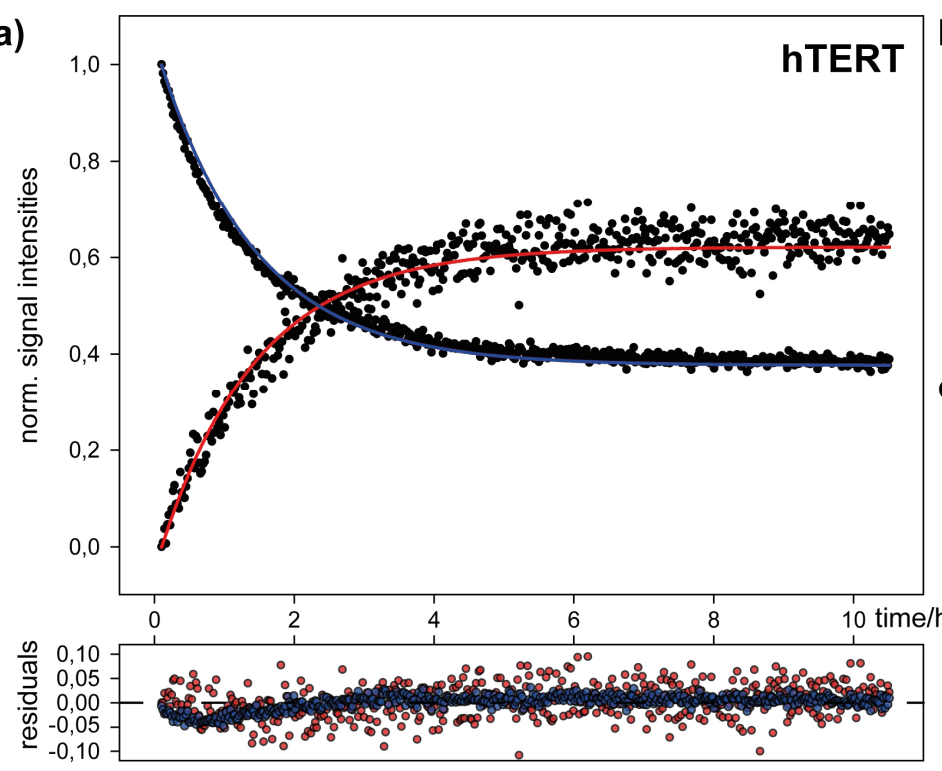

b)
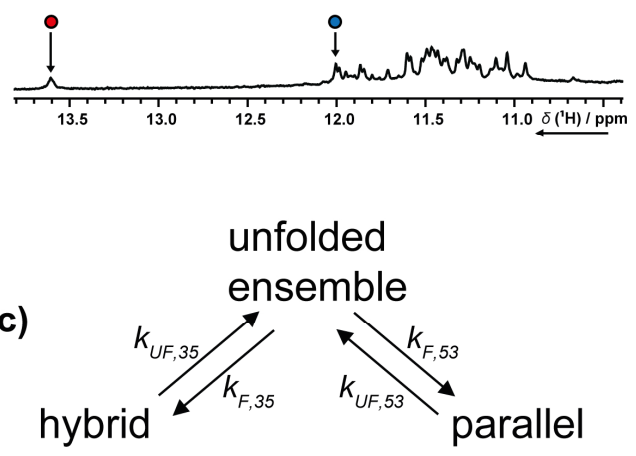

Figure 43: Refolding transition of hTERT G-register isomers. (a) Kinetic traces of individual imino proton signals (b), representative for the two folded conformations, shown in red ( $h T E R T$ hybrid) and blue ( $h T E R T$ parallel). Fitting curves and residuals result from global fitting to the depicted kinetic model (c).

Table 7: Kinetic rate constants for the overall refolding transition $\left(\mathrm{k}_{\mathrm{obs}}\right)$ derived from exponential fitting using non-linear regression and results from global fitting to the kinetic model depicted in Figure 43. For non-linear regression, the calculated standard error is within $5 \%$ as well as the error estimate from fitting residuals.

\begin{tabular}{|c|c|c|}
\hline & $k_{o b s}\left[h^{-1}\right]$ & refolding rate $\left[h^{-1}\right]$ \\
\hline \multirow{4}{*}{ hTERT } & \multirow{4}{*}{$\begin{array}{c}\text { Refolding } \\
0.67\end{array}$} & $\mathrm{k}_{\mathrm{UF}, 35(\mathrm{hybrid})}=1.2$ \\
\hline & & $\mathrm{k}_{\mathrm{F}, 35 \text { (hybrid) }}=491$ \\
\hline & & $\mathrm{k}_{\mathrm{UF}, 53 \text { (parallel) }}=0.43$ \\
\hline & & $\mathrm{k}_{\mathrm{F}, 53 \text { (parallel) })}=277$ \\
\hline
\end{tabular}




\subsection{Activation Energies of G-Register Exchange Transitions}

The energy profiles of the G-register exchange transitions help to get mechanistic insight into the refolding processes of the inherently different G-register isomers in the $c M Y C$ (parallel to parallel) and in the hTERT G4s (parallel to hybrid).

The refolding kinetics have been measured in the range between 288 and $308 \mathrm{~K}$. Arrhenius analysis (Figure 44) of the temperature-dependent rate constants yields an apparent activation energy for refolding (Table 8 ). The apparent activation energies for $c M Y C$ do not significantly differ, if the experiment is started from the caged $c M Y C-18$ 2345-53 or $c M Y C-18$ 2345-33 conformation. For hTERT, the apparent activation energies are larger compared to $c M Y C$, and do differ for the experiments that start either in the hybrid or parallel conformation.
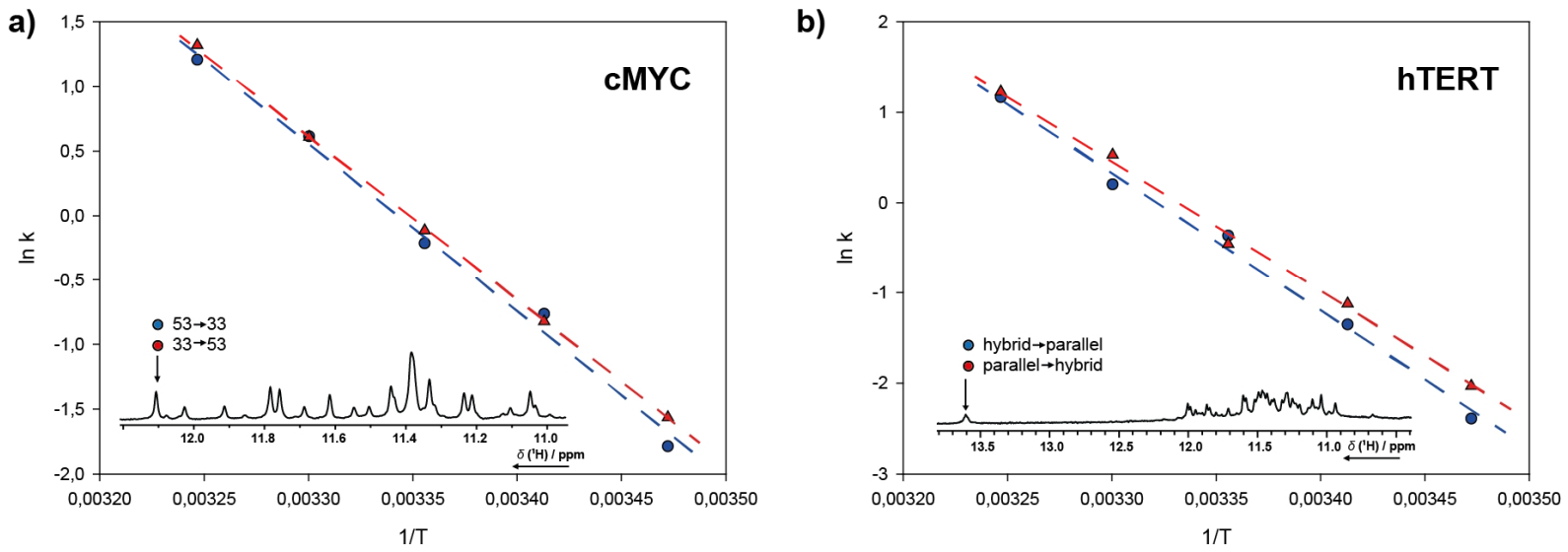

Figure 44: Arrhenius plots of activation energies for refolding of $c M Y C$ (a) and $h T E R T(\mathbf{b})$ G-register isomers. Apparent activation energies have been estimated using linear regression. Data points shown in red and blue represent individual experiments, starting from either of the two caged conformation at each temperature.

Table 8: Apparent activation energies for the refolding reactions between 5'-shifted vs. 3'-shifted parallel conformations ( $c M Y C)$ and parallel vs. hybrid conformations ( $h T E R T)$. Refolding for $c M Y C$ is irrespective of the experimental starting point (caged 53 or caged 33 conformation).

$c M Y C$

hTERT

apparent activation energies

$\left[k J \cdot \mathrm{mol}^{-1}\right]$
107

hybrid: 128

parallel: 120

Melting temperatures $\left(\mathrm{T}_{\mathrm{m}}\right)$ have been determined based on CD melting curves for all of the caged conformations (Figure 45). Melting temperatures are comparable to literature values $\left(c M Y C^{100,101}\right.$ and $\left.h T E R T^{102}\right)$ and reflect the relative population of the coexisting conformations. This is not a trivial finding, as it indicates that for the selected caged conformations no stabilizing or destabilizing effect from the photocages can be observed. The melting curve of the hTERT hybrid conformation is significantly broader than the melting curves from the other G4 conformations and has is not perfectly sigmoidal. This gives indications for a multiphasic, more complex unfolding behaviour of the hybrid 
conformation. The given $\mathrm{T}_{\mathrm{m}}$ is a superposition of those individual steps, which suggests that unfolding steps with higher energetic barriers exist.
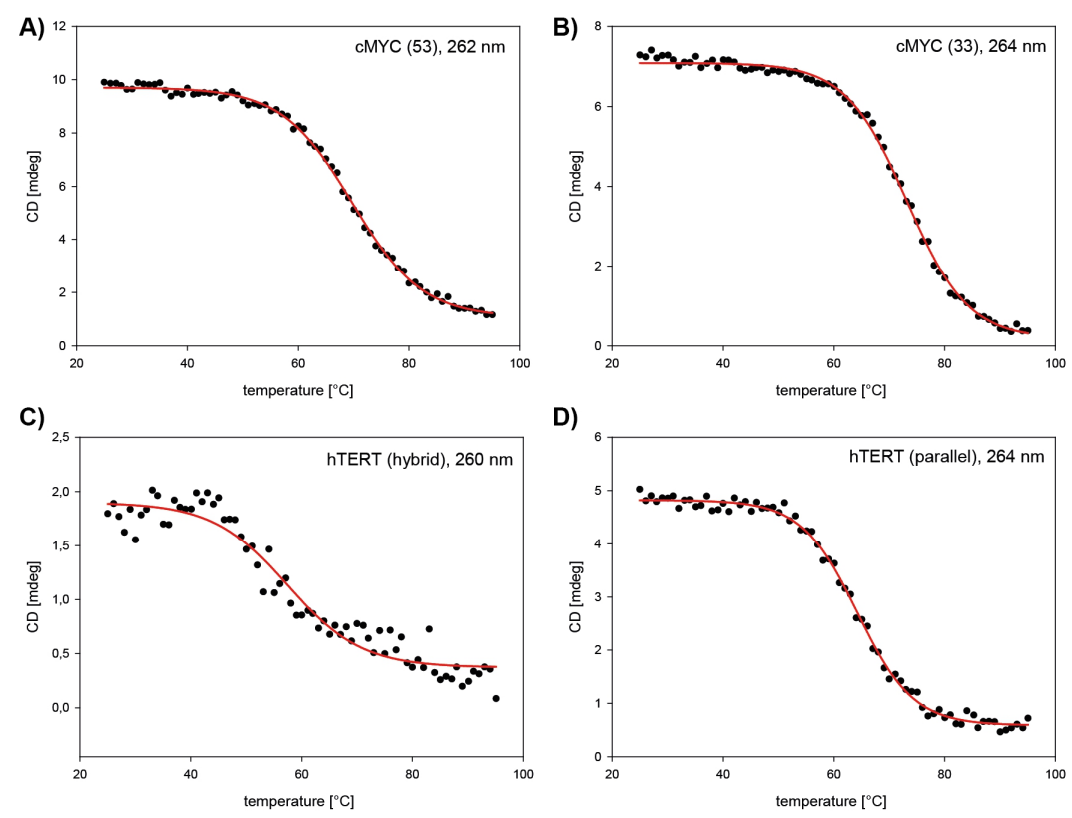

Figure 45: CD melting curves of all investigated caged G4 conformations in the range between $25-95^{\circ} \mathrm{C}$. $\mathrm{T}_{\mathrm{m}} \mathrm{s}$ have been calculated using sigmoidal fitting. The melting curve for $h T E R T$ hybrid (c) indicates multiphasic melting transition.

Table 9: Melting temperatures $\mathrm{T}_{\mathrm{m}}$ of the caged G4 conformations from $c M Y C$ and $h T E R T$, derived from sigmoidal fitting of CD melting curves.

\begin{tabular}{l|l} 
DNA (caged conformation) & $\mathrm{T}_{\mathrm{m}}\left[{ }^{\circ} \mathrm{C}\right]$ \\
\hline$c M Y C 2345-53$ & $69.7 \pm 0.2$ \\
\hline cMYC 2345-33 & $73.2 \pm 0.2$ \\
\hline hTERT (hybrid) & $57.4 \pm 0.9$ \\
\hline hTERT (parallel) & $64.2 \pm 0.2$
\end{tabular}

The apparent activation energies do not allow evaluating directly the degree of unfolding during the refolding process. The calculated apparent activation energies are derived from observable rate constants (exponential fitting) and these relaxation rates cannot be directly assigned to a single rate constant in the proposed kinetic models. They further reflect the energy barriers of the rate-limiting steps during refolding, which is not directly linked to the overall thermal stability of the respective conformations. However, if the thermal stabilities for all conformations would be equal and the same refolding mechanism would be assumed (progressing through the same transitory ensemble), and then comparable activation energies must be expected. Here, opposing trends for these parameters have been observed: the ratio for the mean thermal stabilities for the $c M Y C$ G-register isomers compared to the hTERT G-register isomers is $\approx 1.0: 0.84$, which means that $h T E R T$ has a $16 \%$ decreased thermal stability compared to $c M Y C$. The ratio for the apparent activation energies for refolding is $\approx 1.0: 1.2$, which means that $h T E R T$ requires a $20 \%$ higher activation energy for refolding (20\% slower observable rate constant in refolding). 


\subsection{Folding Initiation in Pre-Equilibrated $\mathrm{K}^{+}$-Solution}

\subsubsection{Dynamics of Thermally-Induced (Un)-Folding of G-Register Isomers}

All of the above-discussed investigations of G-register isomer dynamics have been conducted with oligonucleotides constituted of the wt-sequences. These oligonucleotides have been chemically modified to transiently disturb the native constitution and hence the native distribution of conformation populations. However, the nucleotide sequences of the oligonucleotides can also be modified with single nucleotide mutations. In this way, all relevant G-register isomers of the $c M Y C$ and hTERT G4 can also be isolated and separated permanently in four different oligonucleotides. These stable oligonucleotides also yield single conformations, but cannot refold from one conformation to another. Analysis from thermal melting profiles can yield kinetic information for unfolding and folding of distinct conformations. Rapid cooling or heating causes different cooling and heating profiles in denaturation experiments with shifted apparent melting temperatures. The observation of thermal hysteresis for the folding and unfolding of the G4 conformations provides quantitative information on the underlying rate constants at which thermal folding and unfolding occurs. These experiments based on thermal hysteresis (TH) and their kinetic analysis have been presented by A. K. Mittermaier and co-workers. ${ }^{101,142,737}$ The herein presented results from these experiments and their quantitative analysis have been done by Robert W. Harkness and Christopher Hennecker in the group of A. K. Mittermaier (McGill University, Canada). They are co-authors of the publication Grün et al. 2020. ${ }^{732}$ Experimental details can be found there, the findings are briefly summarized in this chapter for a comprehensive and conclusive discussion.

Melting profiles have been monitored with $U V$-vis absorbance at $295 \mathrm{~nm}$, with scan rates of 1, 2, 3 and $4{ }^{\circ} \mathrm{C} \cdot \mathrm{min}^{-1}$. Based on the analysis of the thermal hysteresis $(\mathrm{TH})$ experiments the refolding process was simulated according to the kinetic models depicted in Figure 46. These simulations yield apparent activation energies and rate constants shown in Table 10. Note, that the kinetic models must include unfolded states, because the experiments report on (complete) thermal unfolding (and folding). This can be in thermodynamic aspects fundamentally different from the isothermal refolding reported from the light-induced NMR experiments.

Table 10: Apparent activation energies and rate constants extracted from simulations for refolding between the G-register isomers in $c M Y C$ and $h T E R T$. The simulations are based on rate constants for folding and unfolding that have been extracted from thermal hysteresis experiments. These experiments cannot account for direct conversion/refolding, but measure two-state transitions between folded and unfolded states.

$$
\text { conformation app.activation energies app.rate constants }
$$

\begin{tabular}{c|ccc} 
& & $E_{\text {relax }}\left[\mathrm{kJ} \cdot \mathrm{mol}^{-1}\right]$ & $k_{\text {relax }}\left[\mathrm{h}^{-1}\right]$ \\
\hline \multirow{2}{*}{ cMYC } & 5'-shifted (53) & $129 \pm 2$ & $0.15 \pm 0.01$ \\
& 3'-shifted (33) & $129 \pm 2$ & $0.15 \pm 0.01$ \\
\multirow{2}{*}{ hTERT } & parallel (53) & $144 \pm 4$ & $0.93 \pm 0.03$ \\
& hybrid (35) & $146 \pm 1$ & $0.33 \pm 0.01$
\end{tabular}


a)

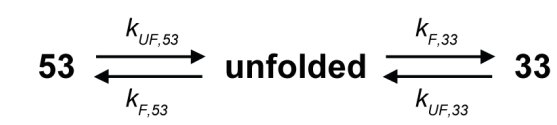

b)

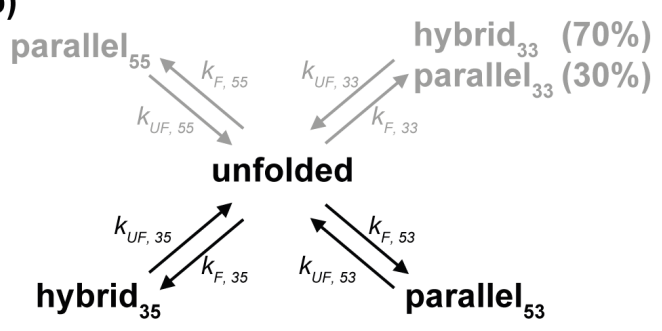

Figure 46: Kinetic models used for the simulation of refolding kinetics between the G-register isomers in $c M Y C(\mathbf{a})$ and hTERT $(\mathbf{b})$. Shown in grey are additional states that have been proposed for $h T E R T$, but are not significantly populated in equilibrium (Figure 42 and Phan et al. ${ }^{102}$ ). Analysis and experimental data for these conformations are discussed in detail in Grün et al.. ${ }^{732}$

For $h T E R T$, the mean of the predicted apparent rate constants calculated for a refolding according to the kinetic model depicted in Figure $46 \mathrm{~b}$, is in good agreement with the NMR derived rates (NMR: $0.67 \mathrm{~h}^{-1}$; TH: $0.63 \mathrm{~h}^{-1}$ ). For $c M Y C$ in contrast, the predicted apparent rates derived from $\mathrm{TH}$ according to the kinetic model depicted in Figure 46 a) are slower by a factor of $\sim 6$. The apparent activation energies calculated from $\mathrm{TH}$ are $\sim 13 \%$ larger for $h T E R T$ and $\sim 20 \%$ larger for $c M Y C$ compared to the NMR derived activation energies for isothermal refolding. Given the very different experimental approaches, the agreement for $h T E R T$ is still remarkable, even though it is significantly different. For $c M Y C$, the apparent activation energies are somehow not comparable anymore for the two experimental results and their underlying models. Both the comparison of kinetic rates and the apparent activation energies are overall in good agreement for $h T E R T$. This implies that the model, which has been proposed for the analysis in both kind of experiments, is suitable to describe the observed refolding process. For $c M Y C$, the drastic differences in TH and isothermal NMR experiments imply that no complete unfolding occurs for the G-register exchange process.

The kinetic and energetic parameters from TH experiments have however not been determined directly, because in these experiments the two conformations are separated by mutations. The primary results from the $\mathrm{TH}$ experiments is folding (and unfolding, not shown) rates for the single conformations, given in Table 11. Most remarkably, the folding rates for both cMYC G-register isomers is approx. $10^{3}$-times faster as the $\mathrm{K}^{+}$-induced folding rates from the NMR experiments $\left(\sim 35 \mathrm{~h}^{-1}\right.$ for 53 and $\sim 25 \mathrm{~h}^{-1}$ for 33 ).

Table 11: Apparent activation energies and rate constants extracted from TH experiments for the thermal folding of Gregister isomers in $C M Y C$ and $h T E R T$.

\begin{tabular}{|c|c|c|c|}
\hline & conformation & $\begin{array}{c}\text { app. activation energies } \\
E_{F}\left[\mathrm{~kJ} \cdot \mathrm{mol}^{-1}\right]\end{array}$ & $\begin{array}{c}\text { app. rate constants } k_{F} \\
{\left[h^{-1}\right]}\end{array}$ \\
\hline \multirow{2}{*}{$c M Y C$} & 5'-shifted (53) & $-75 \pm 4$ & $3800 \pm 700$ \\
\hline & 3'-shifted (33) & $-73 \pm 3$ & $6000 \pm 1000$ \\
\hline
\end{tabular}




\subsubsection{Light-Induced Folding into G-register Isomers}

To scrutinize the drastic differences in the folding rates for thermally induced folding in the $\mathrm{TH}$ experiments and $\mathrm{K}^{+}$-induced folding in the NMR experiments, the photocaging strategy was expanded. An unfolded state in the presence of $\mathrm{K}^{+}$has been prepared, by introducing a total of three $(R)$-NPE photocages to the $c M Y C-2345$-wt 18-mer. This strategy was successful to prevent completely the folding of the $\mathrm{G} 4$ forming oligonucleotide in the presence of $\mathrm{K}^{+}$and at ambient temperatures (Figure $47 \mathrm{~b}$ ). The characteristic region for imino proton signals in slow exchange that shows Hoogsteen base pair interactions does not indicate any kind of pre-folded structures. The photolysis reaction is complete within $4 \mathrm{~s}$ of laser irradiation and the folded state can be recaptured after isothermal folding. The folding reaction here is accelerated by a factor of $\sim 10^{3}$ (mono-exponential fitting yields an approximation of $\sim 6300 \mathrm{~h}^{-1}$ ), compared to $\mathrm{K}^{+}$-induced folding. Folding of the isolated 53-isomer is monophasic in good approximation. This folding relaxation time is in the range of the observed kinetics for thermal folding in the TH experiments (4.6.1).
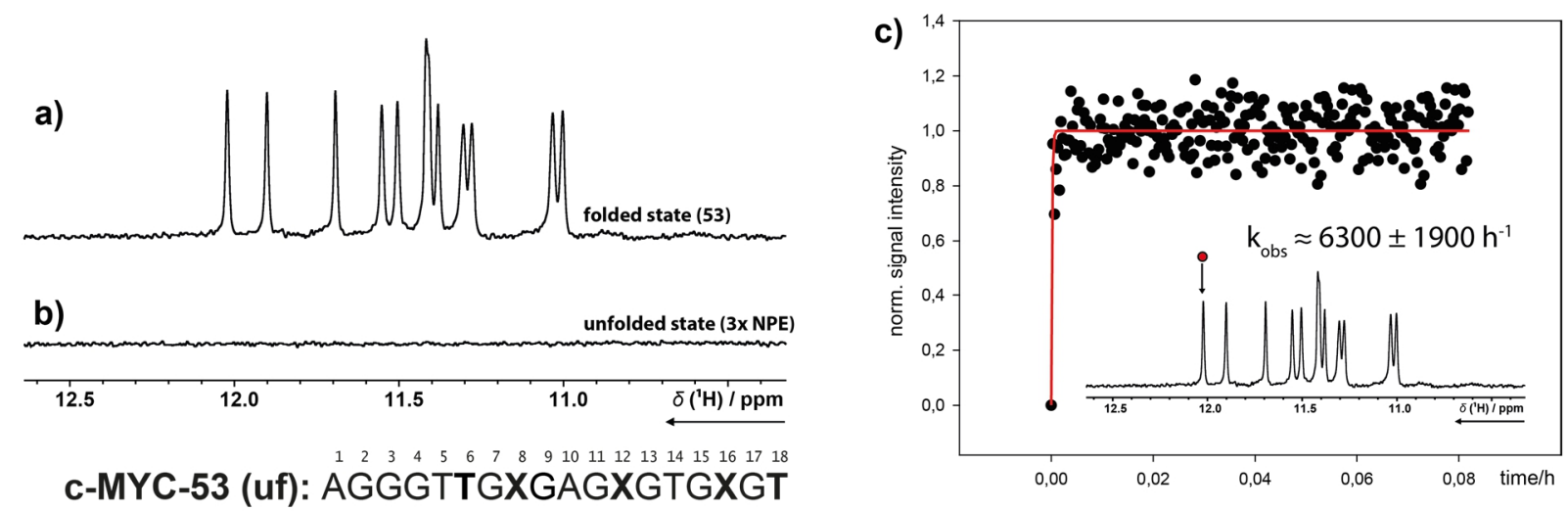

Figure 47: ${ }^{1} \mathrm{H}$ NMR spectrum of a) the 18-mer $c M Y C$ 53-sequence in a native oligonucleotide; and $\left.\mathbf{b}\right)$ with three $(R)$-NPE cages at position G8, G12 and G16 (marked with X in the sequence, conformation-53). c) Kinetic trace for light-induced folding and mono-exponential fitting (red) to yield $\mathrm{k}_{\mathrm{obs}}$.

Two major effects can account for the differences in the observed kinetics. (i) While the initial folding experiment was induced by addition of $\mathrm{K}^{+}$, the folding experiment in Figure 47 and in $\mathrm{TH}$ was initiated in a pre-equilibrated $\mathrm{K}^{+}$solution. (ii) Folding in the $\mathrm{TH}$ experiments and the light-induced folding have been measured on $c M Y C$ sequences with a single isolated G-register isomer (53), while the initial folding experiment (4.2) reports on folding of a sequence that can fold into both G-register isomers 33 and 53.

To quantify these effects, the 18-mer $c M Y C-2345$-wt (X3) that can fold in both G-register isomers has been trapped in an unfolded state following the same photocaging strategy. Figure 48 shows that this sequence can also be trapped in a mainly unfolded state. However, broad signals between 10.9 and $11.5 \mathrm{ppm}$ indicate that in this case some pre-structured conformations can be formed. This nicely highlights the challenge to prevent base pair interactions in G4 forming oligonucleotides. Here, the addition (or better remaining with respect to the wt-sequence) of only a single G-residue enables at 
least some extent of pre-formation. Therefore, a quantification of this experiment (in comparison to other light-induced folding experiments presented within this thesis) must be taken with caution. The fits to the kinetic traces shown in Figure $48 \mathrm{c}$, are meant to represent a trend in the folding kinetics. What can be qualitatively stated is that for the overall folding (integration between $10.8-12.2 \mathrm{ppm}$, black dots) is clearly biphasic.

The separated, conformation-specific traces follow again the same trends observed for the $\mathrm{K}^{+}$-induced folding, with a kinetic overshoot of the 33-isomer and subsequent re-equilibration. The initial folding phase (for overall folding) is finished within the dead time of the experiment, which is approximately $\sim 50 \%$ faster compared to folding of the isolated 53-isomer (Figure 47). The subsequent refolding within the following $\sim 5$ minutes is $\sim 10^{2}$-times faster than the refolding after $\mathrm{K}^{+}$-induced folding. The amplitude of the subsequent, slower refolding dynamics to reach the thermodynamic equilibrium is smaller than for the $\mathrm{K}^{+}$-induced folding experiment.
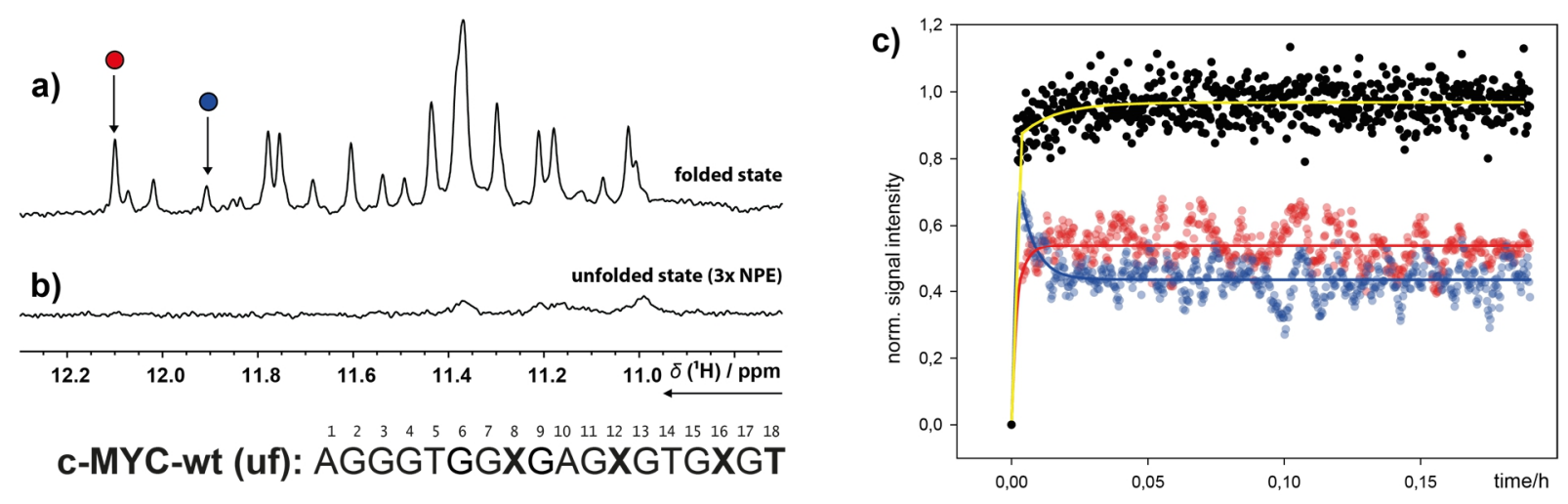

Figure 48: ${ }^{1} \mathrm{H}$ NMR spectrum of a) the 18-mer $c M Y C$ X3-sequence with two possible G-register isomers in a native oligonucleotide; and b) with three (R)-NPE cages at position G8, G12 and G16 (marked with X in the sequence, conformation-53). c) Kinetic trace for light-induced folding and bi-exponential fitting to show the trends in the folding kinetics (yellow, estimate for the relaxation time). Shown in black is the kinetic trace for the overall folding (normalized integration from $10.8-12.2 \mathrm{ppm}$ ). Shown in red (33) and blue (53) are the conformation-specific kinetic traces with exponential fits to show the trend in the refolding, subsequent to initial folding.

\subsection{Conclusions}

The major G-quadruplex conformation of the $c M Y C$ NHE-III $I_{1}$ G4 forming sequence is able to adopt up to four different folded loop-isomers with all-parallel topology that are related by a G-register shift in the G-tract III or V (either 5'-shifted: 5; or 3'-shifted: 3, to yield 53, 33, 35 and 55). The folding and refolding kinetics of the two majorly populated ${ }^{101,142}$ G-register isomers 2345-53 and 2345-33 have been investigated in this chapter. Time-resolved NMR experiments of the folding process that were initiated either by rapid addition of $\mathrm{K}^{+}(4.2)$ or by photolysis of multiple photocages (4.6.2) revealed a kinetic partitioning mechanism for the folding of the two co-existing G-register isomers. Folding is fast and proceeds on two parallel pathways that directly yield the isomeric folded states. Subsequent to this initial phase, a slower refolding takes places with an observable rate constant of $\sim 0.9 \mathrm{~h}^{-1}$. The 
refolding kinetics back to the conformational equilibrium between the two states have been delineated with light-induced experiments of photocaged isolated single conformations (4.3). The kinetics and activation energies (4.5) for two-state cMYC G-register exchange refolding (both parallel conformations) have been compared to two G-register shift related conformations in the hTERT promoter G4 (hybrid and parallel, 4.4). Finally, the isothermal NMR experiments have been crossevaluated with an experimental approach based on thermal hysteresis for rapid thermal folding and unfolding (4.6.1). It was demonstrated that the presented photocaging strategy for conformational selection and conformational suppression of G4 states is a superior approach to unravel the kinetics of G-register exchange dynamics. It allows the observation of undisturbed relaxation dynamics for native, unmodified (after photolysis) oligonucleotides under isothermal and physiological relevant conditions.

The experimental data give striking evidence that the underlying refolding mechanisms for the different types of G-register exchange dynamics are fundamentally different (Figure 49). For $h T E R T$, the evaluation of all presented experiments are in accordance with an unfolding-folding mechanism. The observed kinetics for isothermal refolding of the two G-register isomers in $c M Y C$ is $6 \mathrm{x}$ faster than expected for a complete unfolding-folding mechanism (estimated from thermal hysteresis experiments). A putative species of the transitory ensemble could be a triplex-like strand orientation that allows persisting tetrad scaffolds. The respective G-tract can be imagined as shifting or sliding along this remaining G4 core. However, the real physics of the transitory ensemble are probably better described by compacted coil-like structures. ${ }^{162,163}$

a)

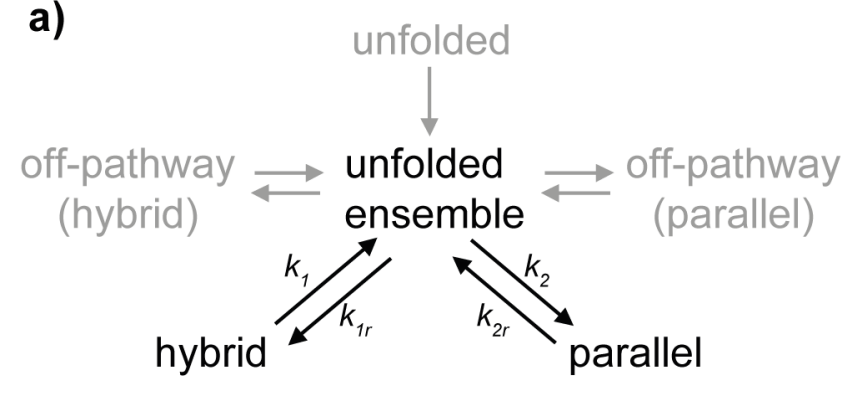

hTERT b)

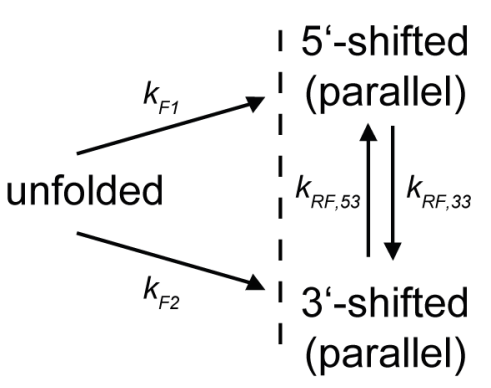

cMYC

Figure 49: Kinetic models for the folding and refolding dynamics of G4-conformations that are related by a formal shift of G-registers with respect to the G4-core tetrads. (a) Model for the hTERT G4 ensemble with a co-existing hybrid and parallel conformation. (b) Model for the $c M Y C-2345$ G4 ensemble with two co-existing parallel conformations.

The slow refolding kinetics for the G-register exchange dynamics exceed the timescales of biologically relevant processes by orders of magnitude (2.2.3). Therefore, the observed off-equilibrium distribution of G-register isomers directly after folding has to be taken into account for a structural evaluation of so-called "major" conformations in polymorphic G4 ensembles. 
G-Register Exchange Dynamics 


\section{Spare-Tire Exchange Dynamics}

\subsection{Overview}

Many G-quadruplexes have additional G-tracts ( $>4)$ that enables them to adopt different G-tract/loop isomers $(2.1 .3,2.3 .3)$. This non-canonical structural feature $(2.1 .3)$ is referred to as spare-tire isomerism. Accordingly, within this chapter the respective isomers will be referred to as "spare-tire isomers".

As discussed and outlined above (4.6.2), folding kinetics following a $\mathrm{K}^{+}$-induced folding are remarkably slower than folding kinetics following folding initiation from uncaging or renaturation from a thermally denatured state. The successful approach of caging a completely unfolded state in the presence of $\mathrm{K}^{+}$was applied to investigate the folding kinetics of the $c M Y C \mathrm{G} 4$ element in more detail.

To separate and deconvolute pure spare-tire exchange from G-register exchange dynamics, the sequence has been adapted to preclude the formation of G-register isomers (Figure 50). This sequence is called "wildtype", with respect to the possibility to form all possible spare-tire isomers.

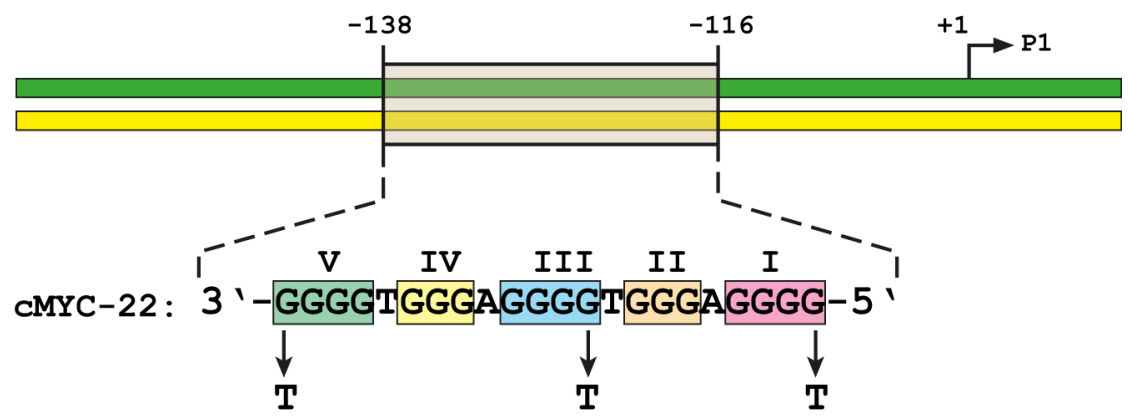

Figure 50: Depiction of the 22-mer sequence from the NHE-III $I_{1}$ in the $c M Y C$ promoter region including G-tracts I-V (numbered from 5'-to-3' direction). Positions that were mutated (G-to-T) to preclude G-register isomers are indicated. Figure has been adapted according to the publications from the Hurley group..$^{99,452}$

The main results presented here have been published in Grün et al. 2021, ${ }^{733}$ accepted manuscript (published online) (doi: 10.1021/jacs.1c01089). All shown figures have been created originally by me (the author of this thesis) and are presented modified or unmodified as compared to the published version: Reprinted (adapted) with permission from J. Am. Chem. Soc. 2021, published online. (doi: 10.1021/jacs.1c01089) Copyright 2021 American Chemical Society. 


\subsection{Light-Induced Folding of Caged Spare-Tire-Isomers}

\subsubsection{Trapping Completely Unfolded Spare-Tire Isomers with Multiple Photocages}

Here, the 22-nt long cMYC sequence was used to investigate the folding of the entire G4 forming sequence that spans all five (1-5) G-rich tracts. This sequence is able to fold into three distinct folded G4 conformations (Figure 51 b), namely the 5'-terminal conformation (5'-1234, abbr. 1234), a longlooped conformation (5'-1245, abbr. 1245) and the 3'-terminal conformation (5'-2345, abbr. 2345). Figure 51 a shows the characteristic imino proton region of the ${ }^{1} \mathrm{H} 1 \mathrm{D}$ spectrum of the $c M Y C-22 \mathrm{wt}-$ oligonucleotide. Wildtype here however should not be misleading, since in fact already mutations have been applied to lock a single G-register isomer for the 2345 conformation (G9T and G22T, resulting in $c M Y C-2345-53$, see Figure 50). Following the distribution of populated conformations for the G4 isomers, 2345 will be called the major conformation and 1234 as well as 1245 will be called minor conformations.

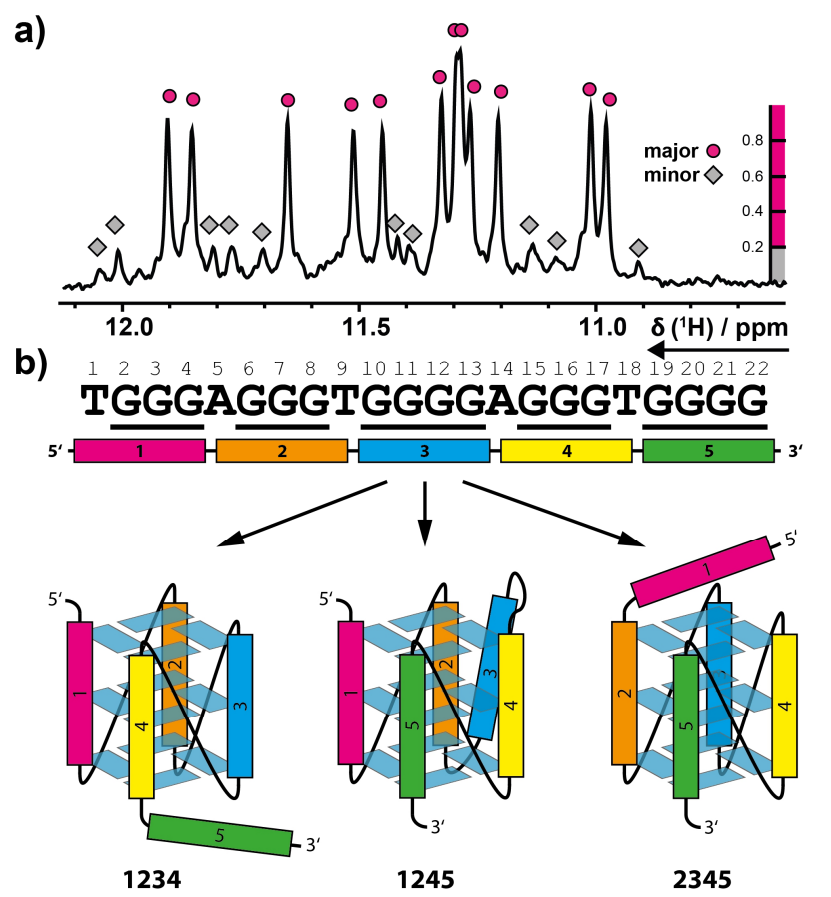

Figure 51: (a) N-H1 imino proton characteristic region of a ${ }^{1} \mathrm{H}-1 \mathrm{D}$ NMR spectrum $(700 \mathrm{MHz}, 298 \mathrm{~K}$, excitation sculpting, $100 \mu \mathrm{M}$ DNA, $5 \mathrm{mM} \mathrm{K}-\mathrm{P}_{\mathrm{i}}$-buffer, $\left.\mathrm{pH}=7.0\right)$ of wt-cMYC-22 and (b) simplified schematic representation of the spare-tire isomers.

To cage the $c M Y C$-22-wt-12345 oligonucleotide in an unfolded state, three (R)-NPE-cages have been placed at the respective positions in G-tract III, IV and V (Table 12). The three different spare-tire isomers $(1234,1245$ and 2345) have been separated and isolated in three different oligonucleotide sequences with G-to-T mutations in the respective G-tracts. In the folded state, these stabilized oligonucleotides can adopt only a single conformation, as the other spare-tire conformations cannot fold anymore. For each of these stabilized oligonucleotides then also up to three $(R)$-NPE-cages have been attached at different G-tracts to trap them in an unfolded state (Table 12). The photocaging strategy was successful to trap the oligonucleotides in an unfolded state. 
Table 12: Sequences and position of $(R)$-NPE-photocages of wildtype and stabilized spare-tire isomers. The wildtype sequence here denotes the 53-isomer only. $(\mathrm{X}=(R)$-NPE-dG $)$

\begin{tabular}{|c|c|c|c|c|c|c|c|c|c|c|}
\hline loop-isomer & & igonuc & leo & tide-s & equer & & & & & \\
\hline & & I & & II & & III & & IV & V & \\
\hline$w t-12345$ & $\mathrm{~T}$ & $\underline{\mathrm{GGG}}$ & A & GGG & TT & $\underline{\mathrm{GX} G}$ & A & $\underline{\mathrm{GXG}} \mathrm{T}$ & T $\underline{\mathrm{GXG}}$ & $\mathrm{T}$ \\
\hline stab.-1234 & $\mathrm{T}$ & $\underline{\text { GGG }}$ & A & GGG & TT & $\underline{\mathrm{GXG}}$ & A & $\underline{\mathrm{GXG}} \mathrm{T}$ & T TTT & $\mathrm{T}$ \\
\hline stab.-1245 & $\mathrm{T}$ & $\underline{\mathrm{GGG}}$ & A & $\mathrm{GX} \mathrm{G}$ & $\mathrm{TT}$ & TTT & A & $\underline{\mathrm{GXG}} \mathrm{T}$ & I $\mathrm{GXG}$ & $\mathrm{T}$ \\
\hline stab.-2345 & $\mathrm{T}$ & TTT & A & GGG & $\mathrm{TT}$ & $\mathrm{GXG}$ & A & $\mathrm{GX} G \quad \mathrm{~T}$ & $T \mathrm{GXG}$ & $\mathrm{T}$ \\
\hline
\end{tabular}

The ${ }^{1} \mathrm{H}$ 1D spectra (Figure 52) show no signals in the characteristic region for N-H1 imino protons with Hoogsteen base pair interactions (10.4 - $12.5 \mathrm{ppm})$. From these spectra, the formation of any pre-folded states can be precluded. For partially folded or pre-folded states, at least broad signals would be expected, arising from interactions in the intermediate exchange regime.

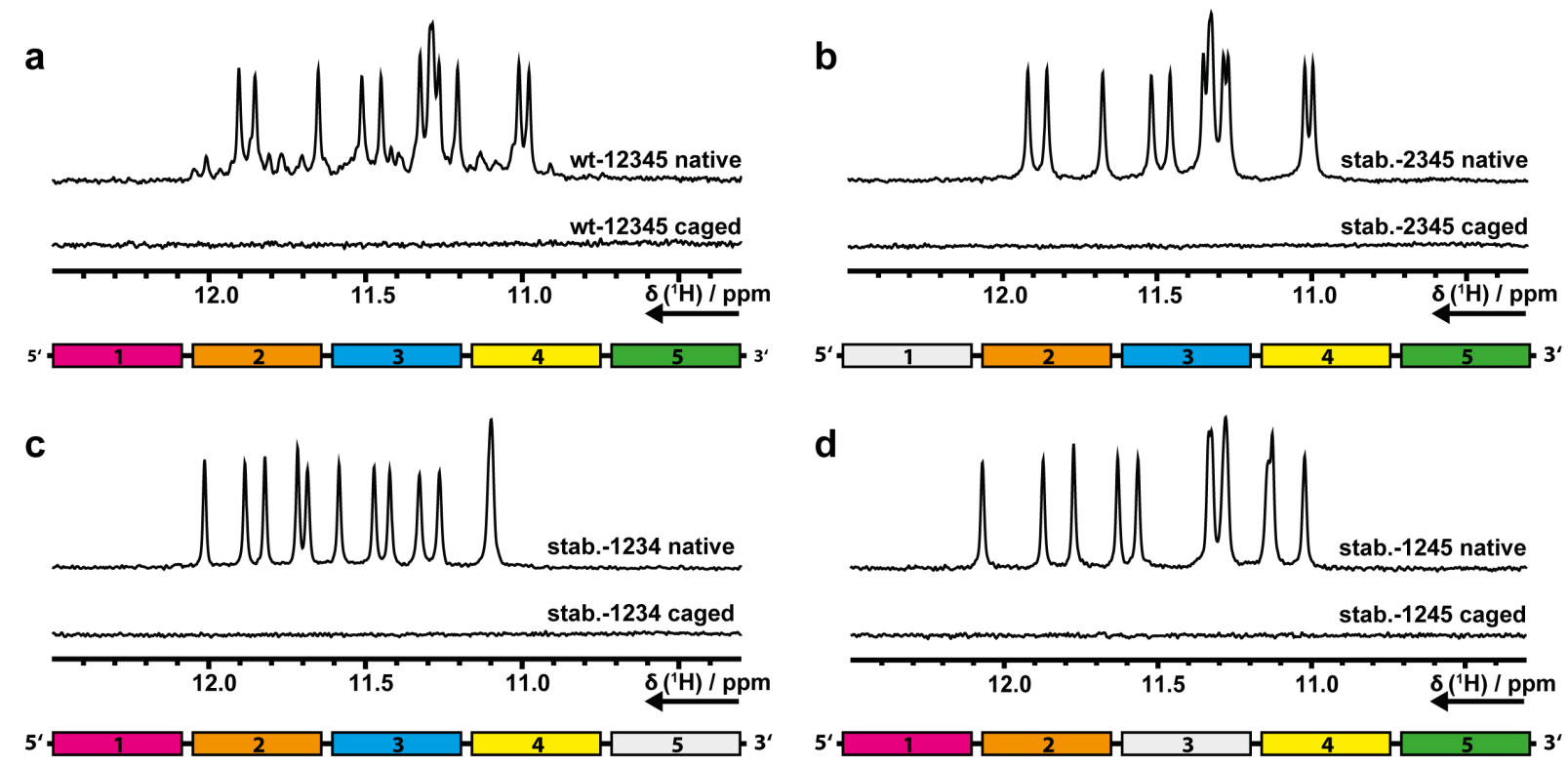

Figure 52: N-H1 region of ${ }^{1} \mathrm{H} 1 \mathrm{D}$ NMR-spectra of caged and native (uncaged) oligonucleotides for cMYC-22 wildtype (a) and spare-tire isomers $(\mathbf{b}-\mathbf{d}),\left(700 \mathrm{MHz}, 298 \mathrm{~K}\right.$, excitation sculpting, $100 \mu \mathrm{M} \mathrm{DNA}, 5 \mathrm{mM} \mathrm{K}-\mathrm{P}_{\mathrm{i}}$-buffer, $\left.\mathrm{pH}=7.0\right)$.

\subsubsection{Light-Induced Folding Kinetics under Pre-Equilibrated $\mathrm{K}^{+}$Conditions}

Starting from these photocaged unfolded states, folding was triggered with $4 \mathrm{~s}$ of laser irradiation (3.4). The folding reactions were monitored using a pseudo-2D experiment that records a series of ${ }^{1} \mathrm{H} 1 \mathrm{D}$ spectra with jump-return-echo water suppression optimized for the detection of imino protons (700 MHz: $\mathrm{d} 19=50 \mu \mathrm{s}$ ). The signals in the spectral region between 10.8-12.1 ppm (see also Appendix 6.2, Figure 67) have been integrated and the normalized signal intensity was plotted to yield kinetic traces for the build-up rate of the G4-specific imino protons (Figure 53). 

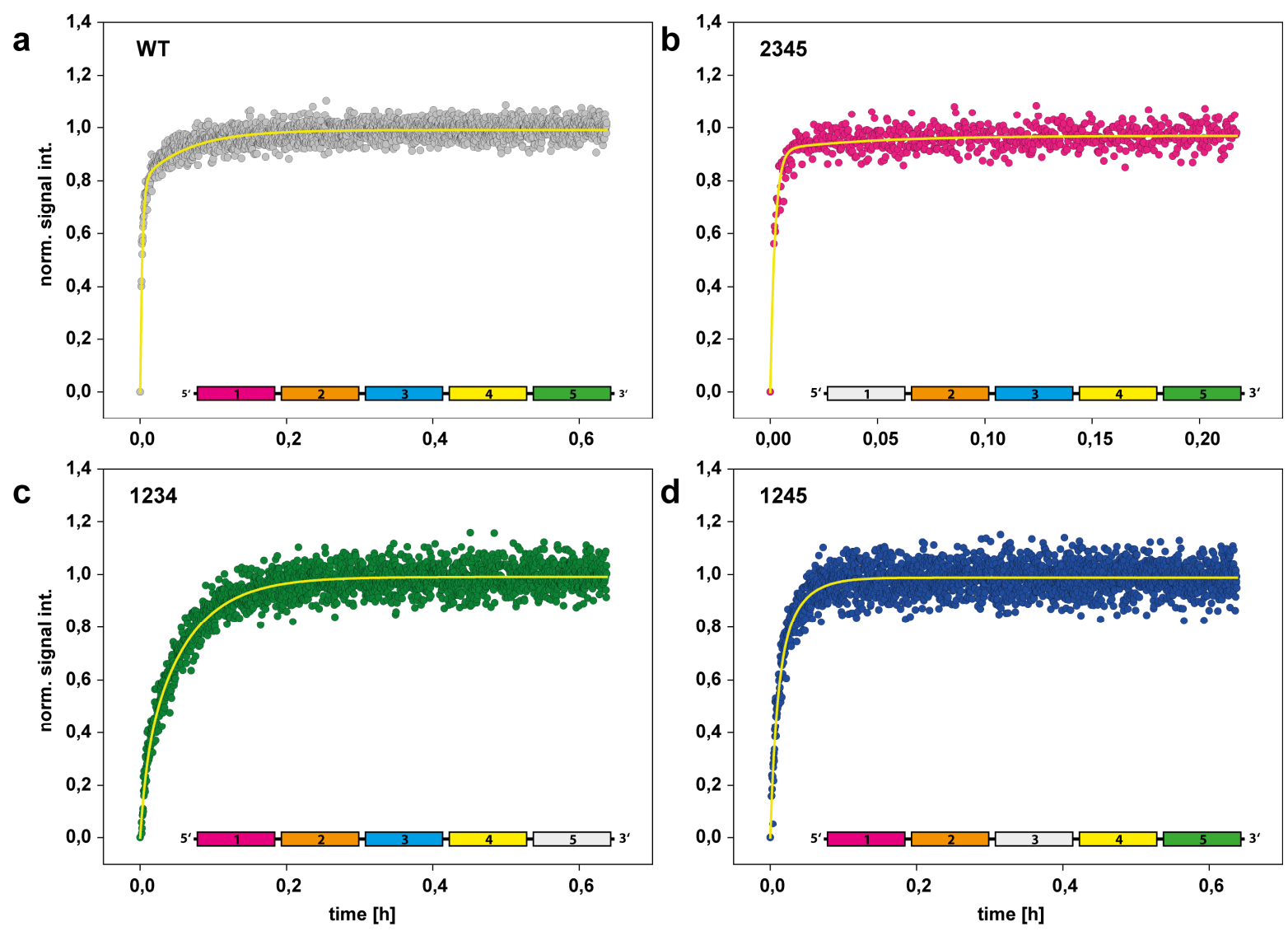

Figure 53: Plots of kinetic traces for light-induced folding of $c M Y C-22$ : wildtype (a, grey), major spare-tire isomer 2345 (b, magenta) and minor spare-tire isomers 1234 (c, green) and 1245 (d, blue). The plots show the normalized signal intensity for imino proton signals in the range between 10.8-12.1 ppm. Fits using bi-exponential non-linear regression are shown in yellow. (100 $\mu \mathrm{M}$ DNA, $5 \mathrm{mM}$ K-P $\mathrm{P}_{\mathrm{i}}$-buffer, $\left.\mathrm{pH}=7.0\right)$.

At room temperature the folding kinetics are sufficiently slow (s-min) to trace them, even after $4 \mathrm{~s}$ of laser irradiation, which is the initial dead time within the experiment. The time resolution between two points ( 1 scan per point) is $\sim 1.155 \mathrm{~s}$. All four folding kinetics (wt and three stabilized isomers) are clearly biphasic, with an initial faster folding regime and a subsequent slower folding regime. This statement is not meant to declare that the underlying folding process is only biphasic, but it can be multiphasic with convoluted kinetic steps. Based on the observable biphasic behaviour, the kinetics have been fitted with bi-exponential non-linear regression, which yields excellent representations for the folding reactions. The observable kinetic rate constants for folding as well as the amplitudes for the folding phases are given in Table 13.

Table 13: Observable kinetic rate constants $\left(\mathrm{k}_{1}, \mathrm{k}_{2}\right)$ for light-induced folding of cMYC-22 spare-tire isomers obtained from bi-exponential fitting. The ratio $\mathrm{k}_{1}: \mathrm{k}_{2}$ describes the relative amplitudes of the folding phases.

\begin{tabular}{llll}
\hline isomer & $\mathrm{k}_{1}\left[\mathrm{~h}^{-1}\right]$ & $\mathrm{k}_{2}\left[\mathrm{~h}^{-1}\right]$ & $\mathrm{k}_{1}: \mathrm{k}_{2}$ \\
\hline stab.-1234 & $110 \pm 16$ & $17 \pm 13$ & $0.26: 0.74$ \\
\hline stab.-1245 & $119 \pm 18$ & $35 \pm 5$ & $0.56: 0.44$ \\
\hline stab.-2345 & $461 \pm 22$ & $22 \pm 8$ & $0.94: 0.06$ \\
\hline$w t-12345$ & $348 \pm 13$ & $16 \pm 1$ & $0.80: 0.20$ \\
\hline
\end{tabular}


The folding kinetics of $c M Y C-22$ have been compared to folding of shortened oligonucleotides, cMYC-18. Here no mutations are needed, because truncation of the 5'-termial or 3'-terminal spare-tire preserves for alternative G-tract interactions. Table 14 shows the sequences for the 5 -truncated (short-1234) and 3'-truncated (short-2345) oligonucleotide. The $c M Y C$-18-short-2345 sequence is comparable to the $c M Y C-18$-X3 sequence used in (4.6.2), but with a G6T mutation, that locks the 53-G-register isomer.

Table 14: Sequences and position of (R)-NPE-photocages of shortened 5'-terminal and 3'-terminal conformations $(c M Y C-18) .(\mathrm{X}=(R)-\mathrm{NPE}-\mathrm{dG})$

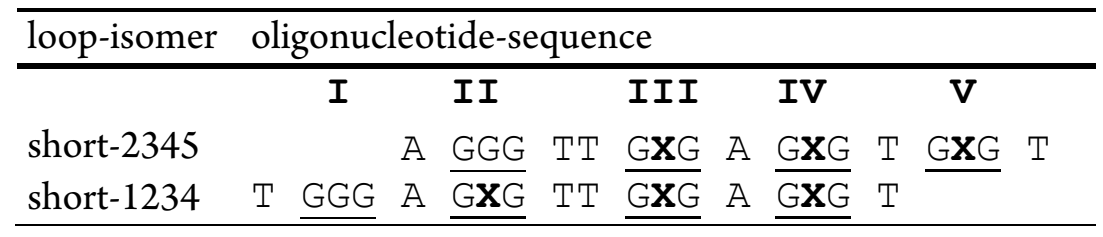

Figure 54 shows the kinetic traces and bi-exponential fits of the light-induced folding. Here, again folding is clearly biphasic with the kinetic rate constants given in Table 15. Noteworthy, the kinetics are significantly accelerated compared to folding of the full-length stabilized spare-tire isomers. The fast folding phase for folding of 2345 cannot be quantified; hence folding is accelerated at least by a factor of $\sim 6.5$ (as estimated from the experimental dead time).
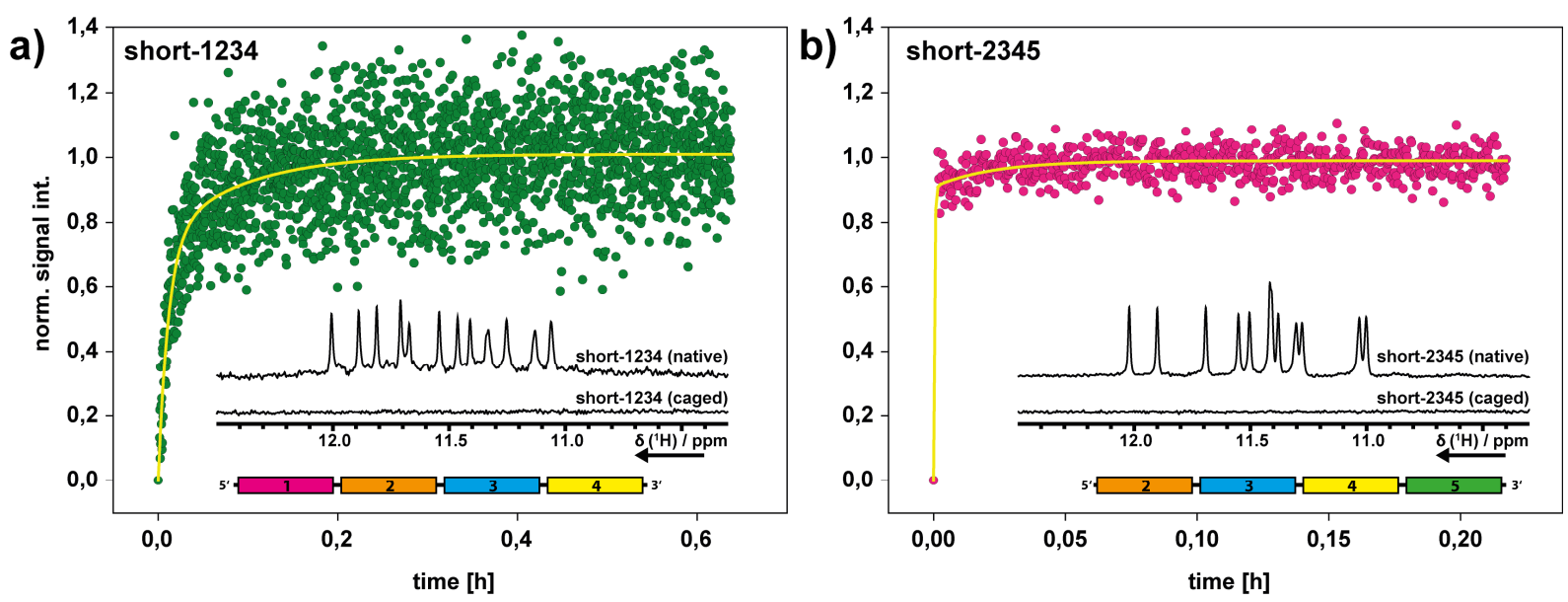

Figure 54: Plots of kinetic traces for light-induced folding of $c M Y C-18$ (shortened 5'-terminal (1234, a) and 3'-terminal $(2345, \mathbf{b})$ conformations). The plots show the normalized signal intensity for imino proton signals in the range between 10.8-12.1 ppm. Fits using bi-exponential non-linear regression are shown in yellow. (100 $\mu \mathrm{M} D N A, 5 \mathrm{mM}$ K- $\mathrm{P}_{\mathrm{i}}$-buffer, $\mathrm{pH}=7.0)$.

Table 15: Observable kinetic rate constants $\left(\mathrm{k}_{1}, \mathrm{k}_{2}\right)$ for light-induced folding of cMYC-18 spare-tire isomers (1234 and 2345-53) obtained from bi-exponential fitting. The ratio $\mathrm{k}_{1}: \mathrm{k}_{2}$ describes the relative amplitudes of the folding phases.

\begin{tabular}{l|l|l|l}
\hline loop-isomer & \multicolumn{1}{l}{$\mathrm{k}_{1}\left[\mathrm{~h}^{-1}\right]$} & $\mathrm{k}_{2}\left[\mathrm{~h}^{-1}\right]$ & $\mathrm{k}_{1}: \mathrm{k}_{2}$ \\
\hline short-2345 (53) & n.d. & $45 \pm 3$ & $0.92: 0.08$ \\
\hline short-1234 & $86 \pm 10$ & $11 \pm 2$ & $0.74: 0.26$ \\
\hline
\end{tabular}




\subsubsection{Intermediate Formation and Folding Pathway for $c M Y C-1234$}

At lower temperatures $(285 \mathrm{~K})$, folding of $c M Y C-1234$ revealed the formation of a long-lived intermediate state. In total eight additional imino proton signals build up after photolysis, mainly shifted to the downfield region ( $>12 \mathrm{ppm}$, Figure $55 \mathrm{a}$ ). The additional signal build up in parallel to the build-up of imino proton signals that are conformation specific for the 1234 conformation (see also Appendix 6.2, Figure 67).

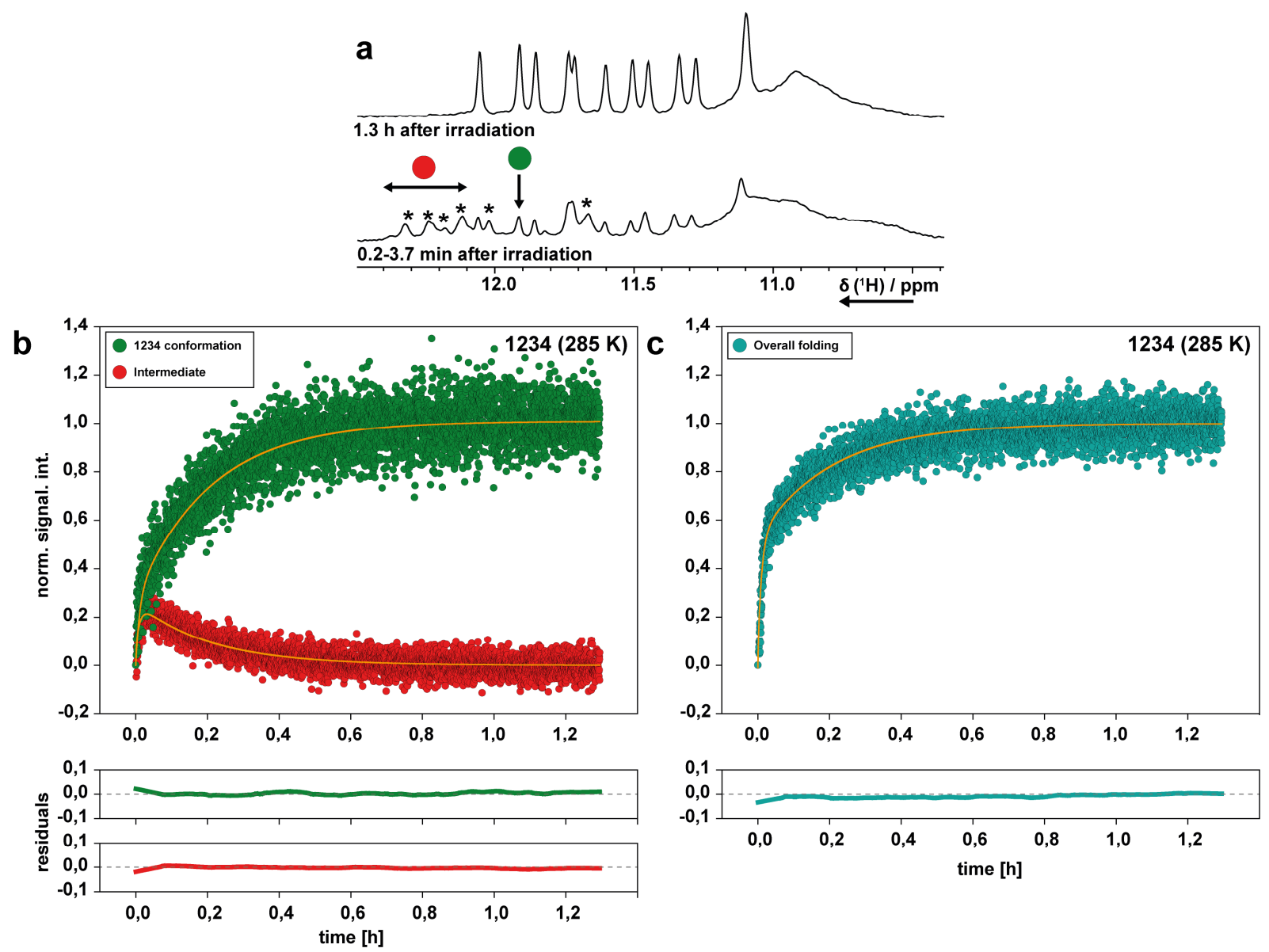

Figure 55: (a) Imino proton NMR spectra of stabilized 1234 at indicated times after light irradiation. (b) Kinetic traces for light-induced folding of stabilized 1234 spare-tire isomer. Shown in green is the trace of only a single conformation-specific signal and shown in red the conformation-specific signals for the intermediate conformation. (c) Shown in turquoise is the kinetic trace for the overall folding $(10.8-12.4 \mathrm{ppm})$. Kinetic fits arise from fitting according to a kinetic model (Figure 56, 3.3) with the respective residual plots shown below.

Figure 55 b shows two kinetic traces from conformation specific (1234: green, intermediate: red, [12.1-12-4 ppm]) imino proton signals. After $\sim 4 \mathrm{~min}$, the kinetic trace for the intermediate conformation reaches a maximum at $\sim 20 \%$ of the normalized total signal intensity in equilibrium. Afterwards it decays over a period of about $1 \mathrm{~h}$ until the peaks completely vanish. Figure $55 \mathrm{c}$ (turquoise) instead shows the integrated peak intensity between 10.8-12.4 ppm, which reflects the overall, conformation independent folding progression. The overall folding is strictly separated in two regimes. The initial folding phase is significantly accelerated in comparison to the conformation specific folding of 1234 alone. The initial folding phase then abruptly passes into a slower phase, after 
the maximum intensity for the intermediate conformation is reached. This kinetic behaviour is expected for parallel folding pathways, which defines the intermediate as off-pathway with respect to the exclusively populated 1234 conformation in equilibrium. The kinetic traces in Figure $55 \mathrm{~b}$ have been fitted to a kinetic model that accounts for parallel folding pathways (Figure 56). The fitting procedure (3.3) used global fitting and yields a prediction for the folding kinetics with the respective kinetic rate constants given in Table 16.

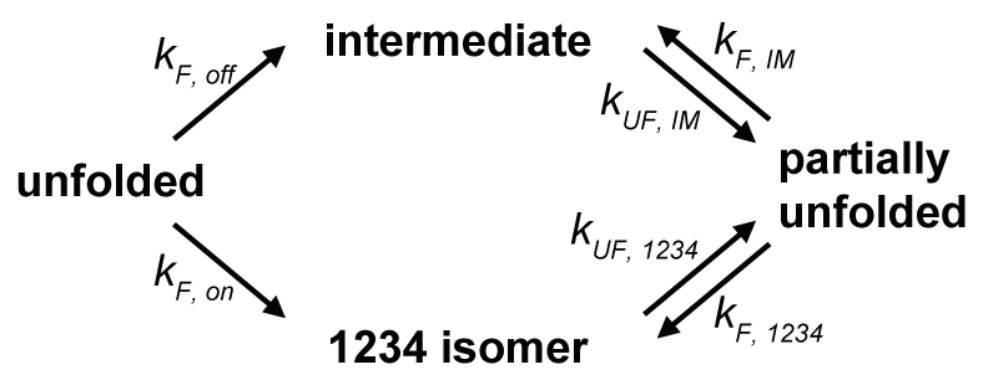

Figure 56: Kinetic model for parallel folding pathways of 1234. The initial folding is irreversible, as the completely (potentially random coil like) unfolded state with photocages cannot be recovered. The ensemble of partially unfolded states accounts for any "not-folded" species.

Table 16: Kinetic rate constants from global fitting for folding of 1234 at $285 \mathrm{~K}$ according to the kinetic model depicted in Figure 56 . The calculated error is within $5 \%$ as well as the error estimate from fitting residuals.

\begin{tabular}{c|c|c|c}
\hline \multicolumn{4}{c}{ fitted rates $\left[\mathrm{h}^{-1}\right]$} \\
\hline \multicolumn{2}{c}{ intermediate } & \multicolumn{2}{c}{1234} \\
\hline $\mathrm{k}_{F, \text { off }}$ & 272 & $\mathrm{k}_{F, \text { off }}$ & 496 \\
\hline $\mathrm{k}_{F, I M}$ & 7.44 & $\mathrm{k}_{F, 1234}$ & 62.7 \\
\hline $\mathrm{k}_{U F, I M}$ & 0.00136 & $\mathrm{k}_{U F, 1234}$ & 29.3 \\
\hline
\end{tabular}

To support the kinetic NMR data, time-resolved CD spectra have been measured. CD-spectroscopy can help to elucidate the secondary structure formation. ${ }^{569,571}$ The CD-cuvette was attached to the $355 \mathrm{~nm}$ laser-setup that has also been used for real-time NMR experiments in a self-designed experimental setup (3.2). After manually triggered laser-irradiation, $\mathrm{CD}$-spectra in the range between 250-300 nm (Figure 57 a, $6 \mathrm{~s}$ interval) or single wavelength measurements at $290 \mathrm{~nm}$ (Figure $57 \mathrm{~b}, 1 \mathrm{~s}$ interval) have been acquired. Peaks in the $\mathrm{CD}$-spectra give hints for the folding topology of G4s: positive bands at $\sim 260 \mathrm{~nm}$ show all-parallel folds and positive bands at $\sim 290 \mathrm{~nm}$ show anti-parallel (or hybrid) folds. ${ }^{170,571}$

The time-resolved CD-spectra show that while the intensity of the band at $260 \mathrm{~nm}$ steadily increases, a small band at $290 \mathrm{~nm}$ again shows an intermediate build-up curve. The kinetic trace of this intermediate band at $290 \mathrm{~nm}$ then was normalized and plotted together with the kinetic trace obtained from time-resolved NMR-spectra. The traces obtained from both methods are completely superimposable, which allows concluding that the topology of the intermediate state is in an anti-parallel conformation. 


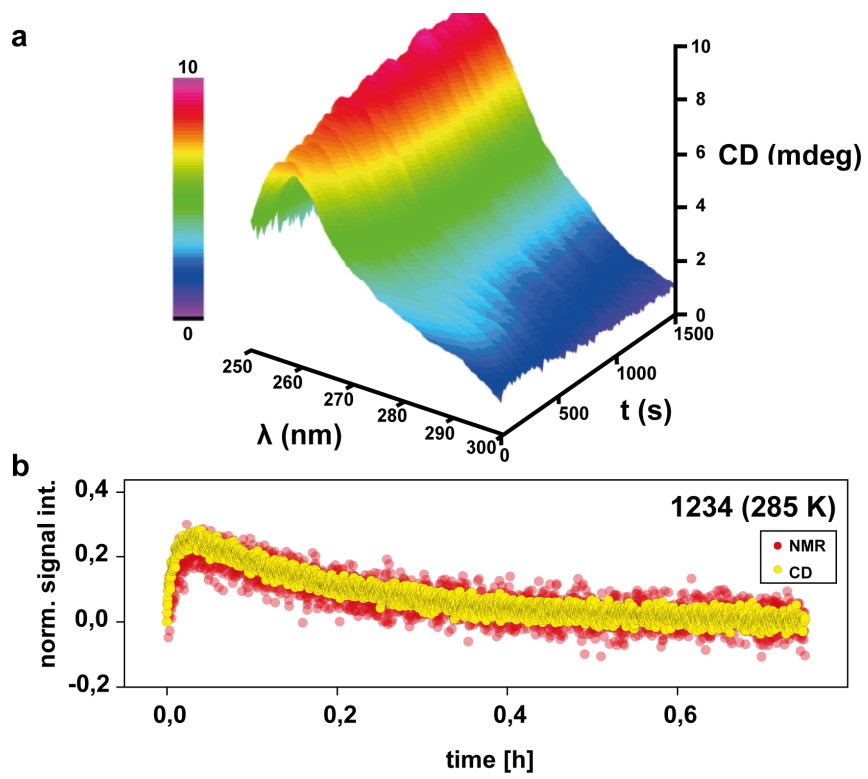

Figure 57: Time-resolved CD data for light-induced folding of 1234. (a) Series of time-resolved CD spectra in the range between $250-300 \mathrm{~nm}$. (b) Normalized overlay of the kinetic trace for an intermediate conformation obtained from NMR $(12.1-12.4 \mathrm{ppm}$ red $)$ and from CD $(290 \mathrm{~nm})$ spectroscopy.

\subsection{Temperature Dependence of Folding}

Light-induced folding has been investigated at variable temperatures in the range between 285 and $310 \mathrm{~K}$. The kinetics for each isomer at every temperature have been fitted with bi-exponential non-linear regression to yield observable rate constants $k_{1}$ and $k_{2}$. Arrhenius plots of the apparent kinetic rate constants are shown in Figure 58 and they show that the temperature difference for folding of each of the isolated spare-tire isomers is fundamentally different.
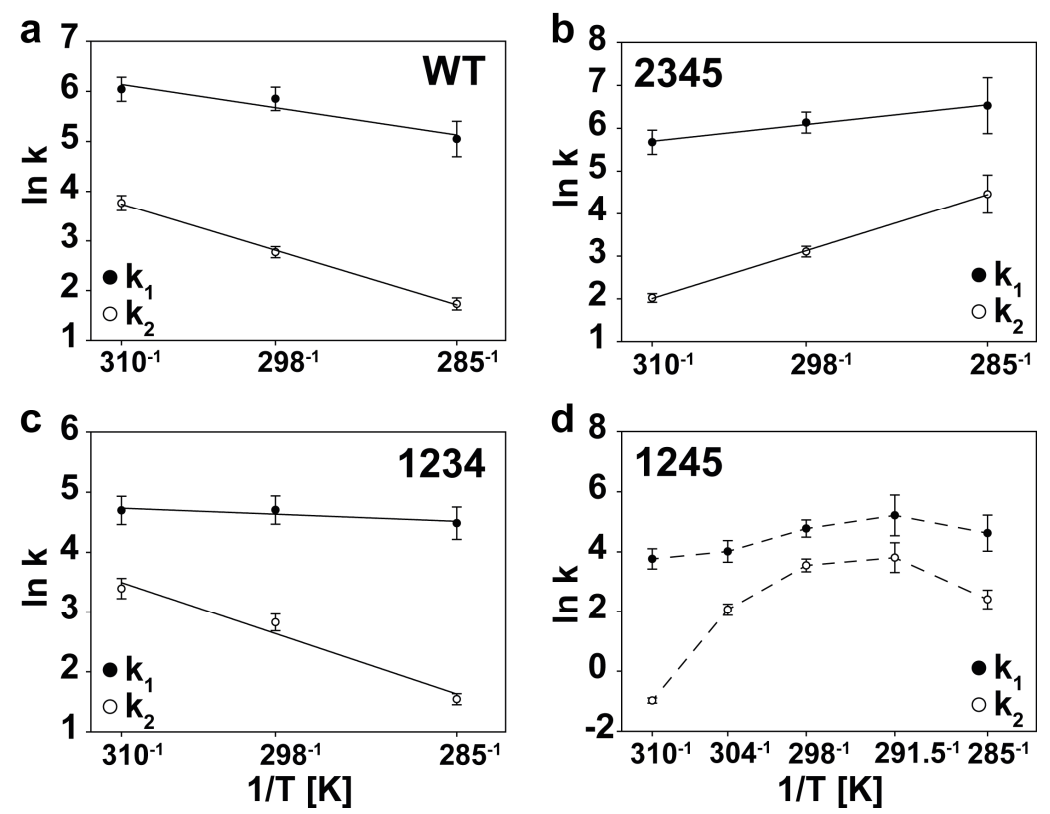

Figure 58: Arrhenius plots for temperature dependent light-induced folding of cMYC-22 spare-tire isomers. (a-c) have been fitted with linear regression, dashed lines in $(\mathbf{d})$ connect the individual experimental points without underlying fitted model to follow the trend. 
The observed apparent activation energy barriers are derived from observable rate constants and cannot be assigned directly to distinct kinetic steps during the complex folding process. They are a superposition of different energy barriers from individual rate constants that are involved in the folding mechanism. These barriers give strong indications for the rate-limiting barriers during folding.

Both the wildtype sequence and the isolated 1234 conformation have a linear Arrhenius correlation with a positive apparent activation energy barrier. The isolated 2345 conformation instead has a linear Arrhenius behaviour with a negative apparent activation energy barrier. The calculated apparent activation energies are given in Table 17. The 1245-conformation shows non-Arrhenius behaviour in the temperature range between 285 and $310 \mathrm{~K}$.

Table 17: Calculated apparent activation energy barriers for $c M Y C-22$ spare-tire isomers, derived from observable rate constants $k_{1}$ and $k_{2}$. The energies are calculated from Arrhenius analysis shown in Figure 58.

\begin{tabular}{l|cc}
\hline isomer & $\Delta \mathrm{E}_{\mathrm{A} 1}\left[\mathrm{~kJ} \cdot \mathrm{mol}^{-1}\right]$ & $\Delta \mathrm{E}_{\mathrm{A} 2}\left[\mathrm{~kJ} \cdot \mathrm{mol}^{-1}\right]$ \\
\hline stab.-1234 & $6.4 \pm 3.5$ & $54.9 \pm 9.9$ \\
\hline stab.-1245 & - & - \\
\hline stab.-2345 & $-25.0 \pm 2.3$ & $-71.8 \pm 1.4$ \\
\hline$w t-12345$ & $29.6 \pm 8.9$ & $59.7 \pm 2.4$ \\
\hline
\end{tabular}

The positive activation energy barrier for folding of the stab.-1234 isomer can be explained with the formation of off-pathway intermediate conformations that need to be unfolded. The observed anti-parallel species presented presumably gives major contributions to the observed total energy barrier, but the activation energy does not necessarily reflect the unfolding energy of only this NMR-visible conformation.

The fast folding relaxation time of the isolated stab.-2345 isomer and the negative apparent activation energy barrier, indicate a mainly entropically driven, close to funnel-like folding process for this conformation. The conformational space during folding is drastically reduced for this sequence, since both the formation of G-register isomers and the competing spare-tire isomers is precluded. Nevertheless, the folding kinetics are remarkably slower than those obtained for the 18-mer cMYC-2345 sequences (4.6.2 and Figure 54; both with and without possible G-register formation). This points to (i) an influence of the flexibility of the 5'-tail and (ii) possible transient base pair interactions with the 5'-elongated nucleotides. These interactions are precluded in the folding experiments of the short $c M Y C-2345$ oligonucleotides, which further guides an optimized folding process.

Folding of the 3'-terminal (2345) and 5'-terminal (1234) conformations mark limiting regimes for either (i) pronounced kinetic partitioning with intermediate formation or (ii) close to funnel-like folding. The non-Arrhenius temperature dependence for the isolated stab.-1245 isomer indicates that 
here folding takes place in an interesting junction region on the folding energy landscape. At lower temperatures, folding is rate limited by unwinding kinetic traps with energy barriers, while at higher temperatures the process gets more entropy driven, with only a negative observed folding energy barrier.

Different kind of possible off-pathway interactions and transient kinetic traps might be present in the folding of the wt-sequence that shows a positive apparent activation energy barrier. This energy reflects refolding barriers from any possible microstates in the large conformational space of the wt-sequence that have to be overcome. To examine, if these off-states possibly involve fully folded minor conformations (spare-tire isomers 1234 and 1245), refolding from these states was investigated.

\subsection{Light-Induced Refolding of Caged Meta-Stable Minor Conformations}

\subsubsection{Conformational Selection}

In chapter 4.3.1 a strategy to trap and isolate single folded G4 conformations has been presented using site-specific suppression of Hoogsteen-interactions with $(R)$-NPE-photocages. This strategy has been applied to trap the minor conformations 1234 and 1245 . Figure 59 shows the $1 \mathrm{D}{ }^{1} \mathrm{H}$ NMR spectra in the relevant Hoogsteen base paired imino proton ppm-range for caged wt-1245 (G12 caged, b) and wt-1234 (G20 caged, c) and a spectrum of the native (uncaged) wt after complete relaxation (a). The spectra clearly show that the conformational selection was successful and that single conformations could be isolated. The caged wt- 1245 however shows additional imino proton signals (marked with \#) that might indicate the formation of a second (or partially folded) conformation.

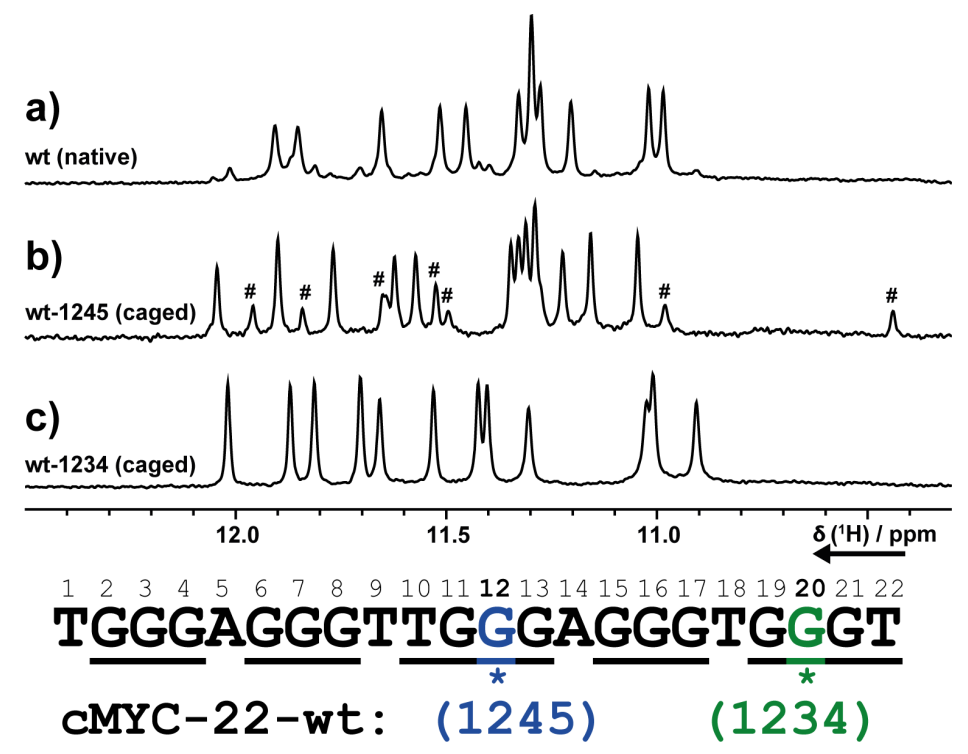

Figure 59: Trapped minor conformations as meta-stable states of cMYC-wt-1234 (G20 caged) and cMYC-wt-1245 (G12 caged). Before (b, c) and after uncaging (a, complete relaxation). (700 MHz, $298 \mathrm{~K}$, excitation sculpting, $100 \mu \mathrm{M}$ DNA, $5 \mathrm{mM} \mathrm{K}-\mathrm{P}_{\mathrm{i}}$-buffer, $\mathrm{pH}=7.0$ ). $\left(^{*}\right)$ indicates caging positions to yield 1245 (blue) or 1234 (green). (\#) indicates an additional set of signals for the photocaged conformation. 
Figure 60 shows a comparison of the caged spectrum (lower) and a spectrum directly after uncaging (upper, $4 \mathrm{~s}$ laser irradiation $+1.15 \mathrm{~s}$ acquisition time). The marked, additional signals are completely vanished, before any kind of spectral changes can be observed. Similar observations were made for the caged parallel-hTERT conformation (chapter 4.4, Figure 55). It cannot be completely ruled out that these kind of signals arise from partially unfolded or otherwise disturbed folded conformations. However, two findings strongly oppose that assumption: (i) the very sharp linewidth that indicate a defined state in the slow exchange regime; and (ii) the very fast decay of the signals in comparison to even the fastest folding, unfolding or refolding transitions for all of the investigated G4 dynamics. Furthermore, this would imply either that these "destabilized" conformations, after uncaging, convert directly to the completely folded conformation or that they completely unfold. If that were correct, a rapid folding of a stable G4 conformation must be expected. The NMR-spectra rule that out. Therefore, a likely explanation for these signals could be alternative arrangements (e.g. stacking) of the $(R)$-NPE-residues that cause perturbed chemical shifts. This explanation would be in line with a moderate shift of the imino proton signals after uncaging that has been observed for the trapped G4 conformations.
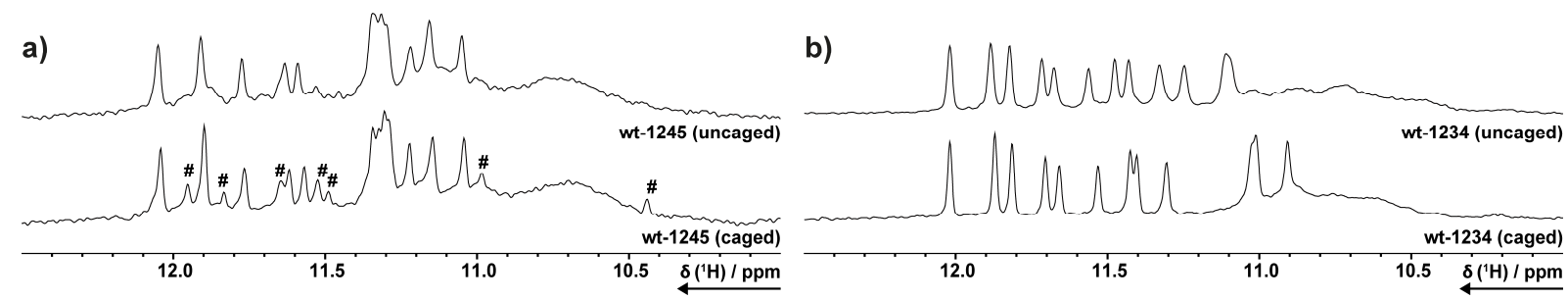

Figure 60: Trapped minor conformations as meta-stable states of $c M Y C$-wt-1245 (a) and cMYC-wt-1234 (b). Before (lower spectra) and directly after uncaging (upper spectra) $(700 \mathrm{MHz}, 298 \mathrm{~K}$, jump-return-echo, $100 \mu \mathrm{M}$ DNA, $5 \mathrm{mM}$ $\mathrm{K}-\mathrm{P}_{\mathrm{i}}$-buffer, $\left.\mathrm{pH}=7.0\right)(\#)$ indicates an additional set of signals for the photocaged conformation.

\subsubsection{Refolding Kinetics}

After quantitative uncaging, the native oligonucleotides are released, persisting in their initially folded G4 conformation. No additional imino proton signals were observed within a short time after photocleavage of the caging-groups, which would indicate the presence of additional conformations. Hence, the starting point of the experiments represents the unperturbed, native state of the single spare-tire G4 conformations. This exclusive population of the minor conformations could otherwise not be observed for the wildtype $c M Y C$-sequence.

The initial conformations then decay over a period of several hours, while signals for the major conformation 2345 rise up (Figure 61). The kinetics for both the unfolding of the minor conformations and the subsequent folding of the major conformation are correlated and progress coherently. The observable rate constants have been obtained from a global fit of both traces with double mono-exponential non-linear regression (Table 18). 
At room temperature, a complete refolding of the minor conformations to the major conformation was observed. Hence, the minor conformations are completely meta-stable. Arrhenius analysis of temperature dependent refolding allows an estimate for the apparent activation energy of refolding (Table 18).
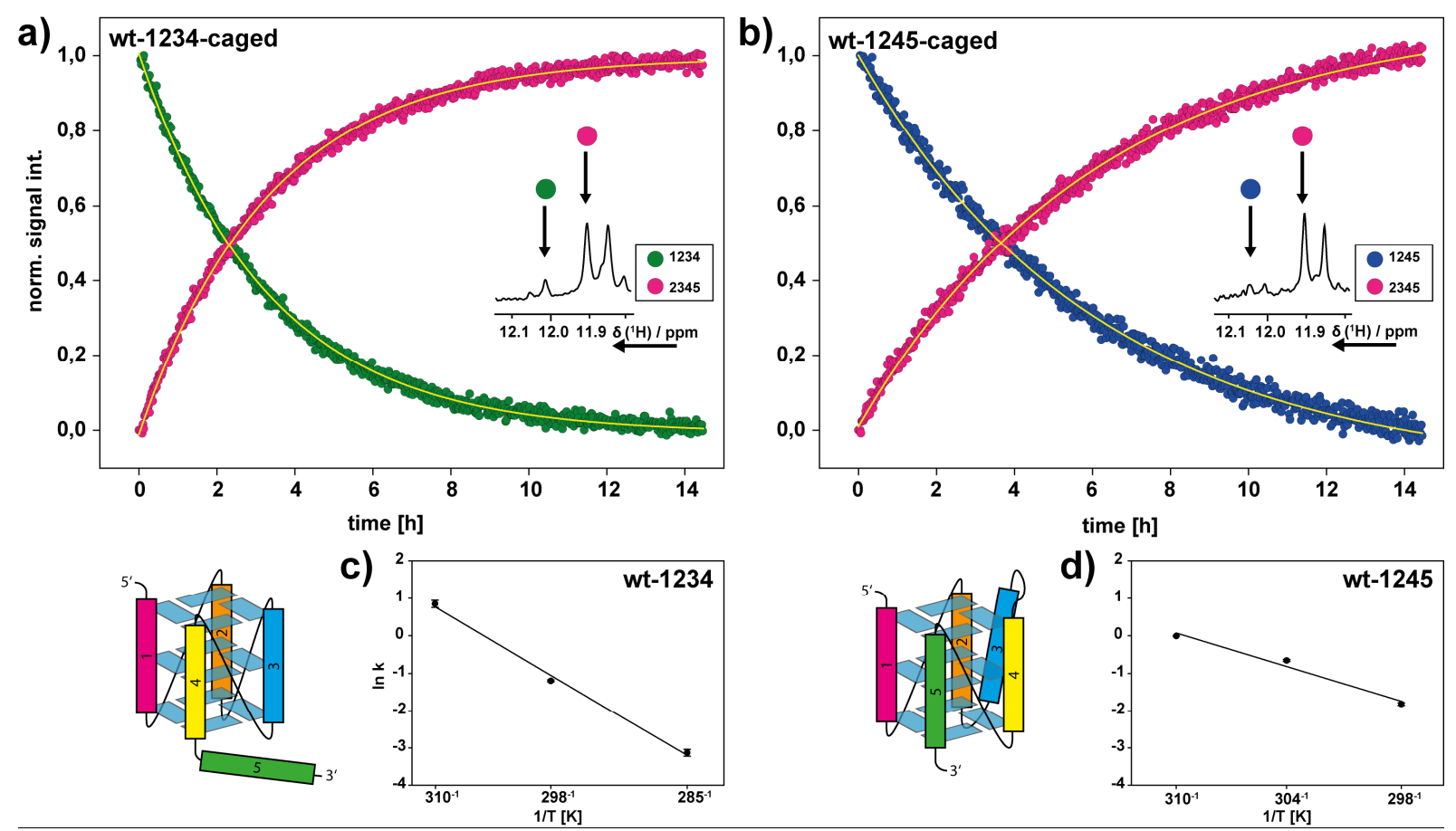

Figure 61: Refolding transition of trapped meta-stable cMYC-22 spare-tire isomers 1234 and 1245 at 298 K. (a, b) Kinetic traces of individual imino proton signals, representative for the two folded conformations (magenta: 2345, green: 1234, blue: 1245). Fitting curves from global mono-exponential regression are shown in yellow. (c, d) Arrhenius analysis with linear regression to calculate apparent activation energies for refolding (Table 18).

Table 18: Observable rate constants for refolding of trapped conformations 1234 and 1245 from the 22-mer $c M Y C$-wt oligonucleotide. Apparent activation energies have been calculated from Arrhenius analysis (1234: $285-310 \mathrm{~K} ; 1245$ : $298-310 \mathrm{~K})$.

\begin{tabular}{c|c|c}
\hline loop-isomer & $\mathrm{k}_{\mathrm{obs}}\left[\mathrm{h}^{-1}\right]$ & \multicolumn{1}{c}{$\Delta \mathrm{E}_{\mathrm{A}}\left[\mathrm{kJ} \cdot \mathrm{mol}^{-1}\right]$} \\
\hline caged-1234 & $0.302 \pm 0.006$ & $116 \pm 8$ \\
\hline caged-1245 & $0.163 \pm 0.001$ & $116 \pm 19$ \\
\hline
\end{tabular}

For the 1234 conformation, a complete refolding was observed even at $285 \mathrm{~K}$. For the 1245 conformation, at lower temperature $(285 \mathrm{~K})$, no refolding and no formation of conformation 2345 was observed (Figure 62). This is unexpected with regard to the calculated apparent activation energies of 1234 and 1245. The activation energy barriers however, reflect only the rate-limiting step for the unfolding of a certain conformation. The greater degree of required rearrangements for the 1245 conformation compared to 1234, might explain why 1245 has an increased lifetime. Irrespective of the same heights for the energy barriers that have to be overcome for unfolding to the transitory ensemble for both minor conformations, presumably 1245 is kinetically trapped. 


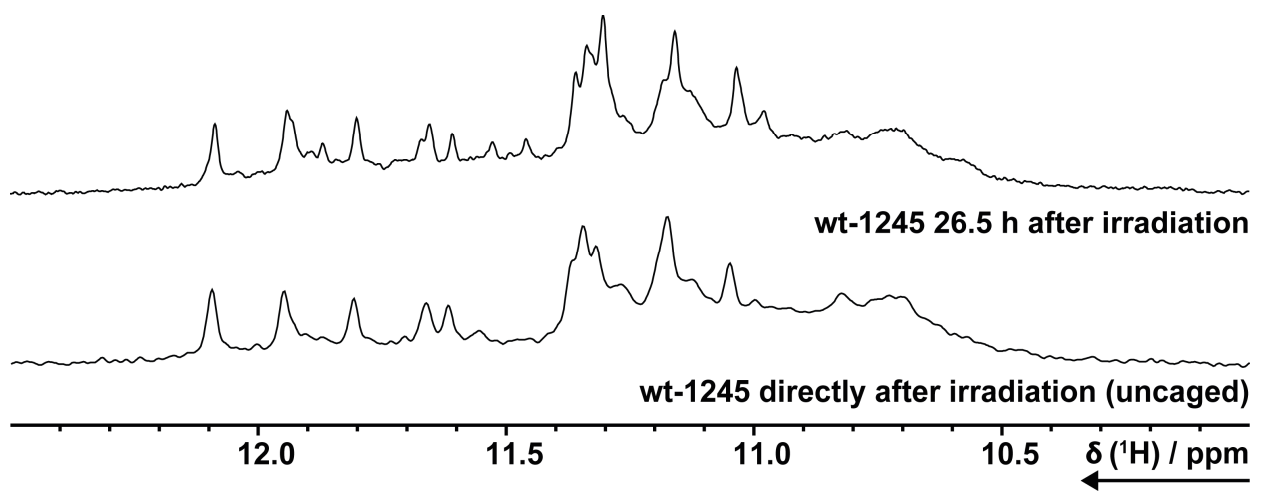

Figure 62: Trapped minor conformation of $c M Y C$-wt-1245 at $285 \mathrm{~K}$. Before (lower spectrum) and after directly after uncaging (upper spectra) $(700 \mathrm{MHz}, 298 \mathrm{~K}$, jump-return-echo, $100 \mu \mathrm{M}$ DNA, 5 mM K-P -buffer, pH=7.0)

\subsection{Conclusions}

The G4 forming sequence in the $c M Y C$ NHE-III 1 is able to adopt up to three different folded loopisomers with all-parallel topology that incorporate different G-tracts into tetrad-formation (either 5 '-1234; 5'-1245 or 5'-2345). The folding and refolding kinetics of these spare-tire isomers have been investigated in this chapter. Time-resolved NMR experiments of the folding process that were initiated by photolysis of multiple photocages (5.2) revealed inherently different folding kinetics. Folding was investigated for separated and stabilized single conformations (1234, 1245 and 2345) and for the wildtype-sequence that is able to adopt all spare-tire conformations (but only a single G-register conformation). Folding of all spare-tire isomers is biphasic and proceeds on multiple parallel pathways that yield the isomeric folded states. Analysis of the temperature dependence of the folding reactions (5.3) reveals different manifestations of the underlying kinetic partitioning mechanisms. The minor conformations 1234 and 1245 have been isolated with a trapping strategy for conformational selection (5.4). The preparation of this single-conformation, off-equilibrium populations revealed that both minor conformations are meta-stable with respect to the major $c M Y C-G 4$ conformation 2345.

Folding kinetics of the wt-sequence could be disentangled as a superposition of the folding kinetics of the minor conformations ( $20 \%$ populated in the wt) and the major conformation ( $80 \%$ populated in the wt). The major conformation $c M Y C-2345$ folds via a close to funnel-like pathway with optimized kinetics. For the minor conformation $c M Y C-1234$, the formation of an anti-parallel off-pathway conformation was demonstrated both with time-resolved NMR and with CD experiments (5.2.3). The minor conformation $c M Y C-1245$ showed an unprecedented non-Arrhenius temperature dependence in the folding kinetics, which is presumably linked to the increased flexibility of the long internal loop that separates the 5'-terminal and the 3'-terminal parts of the tetrad-constituting parts of the oligonucleotide. Thus, the observed inherently different folding dynamics for the formation of sparetire G4 conformations demonstrate markedly different limiting regimes on the folding energy landscape for structurally very similar parallel G4s emerging from the same oligonucleotide sequence. 
Spare-Tire Exchange Dynamics 


\section{The Dynamic Behaviour of the $c M Y C$ Promoter G-Quadruplex}

\subsection{Folding Pathways for the 22 nt cMYC Full Length Sequence}

\subsection{1 $\mathrm{K}^{+}$-Recruitment During Folding}

Folding has been investigated with several methods within this thesis, using time-resolved NMRspectroscopy, time-resolved CD-spectroscopy and a thermal hysteresis approach with UV detection. To obtain kinetic information with these techniques, different approaches were used to prepare unfolded states. In chapter 4.2, $c M Y C \mathrm{G} 4$ folding has been initiated by addition of $\mathrm{K}^{+}$-ions, while in chapters 4.6, and 5.2 and folding has been initiated under pre-equilibrated $\mathrm{K}^{+}$-conditions (thermal denaturation and photocaging). Strikingly, the folding kinetics for $\mathrm{K}^{+}$-induced folding deviate by several orders of magnitude, when compared to the other methods.

$\mathrm{K}^{+}$-induced folding has been used in numerous studies ${ }^{144,167,170}$ on $\mathrm{G} 4$ folding and the findings presented in these studies and within this thesis raise no doubt that this is a legitimate approach to study these processes. However, the recruitment and coordination of $\mathrm{K}^{+}$-ions has a significant effect on the overall folding dynamics, which is a direct conclusion from the presented folding kinetics. Experimental approaches can only describe macrostates (if any), but no microstates that in sum define the conformational entropy of G4 folding. Hence, the folding kinetics do not allow speculating about the reasons for the decelerated folding, after addition of $\mathrm{K}^{+}$. Although it seems reasonable to assume that the conformational space (conformational energy landscape) of the unfolded oligonucleotides is fundamentally different for (i) thermal denaturation, (ii) destabilization with photocages or (iii) $\mathrm{K}^{+}$-free conditions. The contributions of entropy and enthalpy for removing the hydrate shells from free vs. DNA-coordinated $\mathrm{K}^{+}$-ions may add important energetic aspects that alter the folding pathways. Insights for the binding properties of cations to G-quadruplexes have been obtained from theory ${ }^{738}$ and mass spectrometry ${ }^{167,739}$. Especially ${ }^{15} \mathrm{NH}_{4}{ }^{+}$has been extensively studied with $\mathrm{NMR},{ }^{93,740-747}$ but also G4-related experiments have been reported for a direct NMR detection ${ }^{748,749}$ of e.g. ${ }^{23} \mathrm{Na}^{+},{ }^{39} \mathrm{~K}^{+}$or investigation of ion coordination sites with cross-correlated relaxation rates. ${ }^{750}$ Further NMR experimental data and insights from computational methods and simulations will be needed to elucidate the role of $\mathrm{K}^{+}$-binding during folding.

This is of particular importance if $\mathrm{K}^{+}$-coordination becomes rate limiting during folding in a way that e.g. $\mathrm{K}^{+}$-coordination does not happen concerted on both inter-tetrad "binding sites". ${ }^{167,751}$ In this regard, the misfolded or partially folded states proposed by Marchand and Gabelica based on mass spectrometry ${ }^{167}$ have to be evaluated carefully. Here, the authors describe, unlike in other folding studies, a step-by-step titration with substoichiometric amounts of $\mathrm{K}^{+}$. If the lifetimes (or better: $d$ well times) for this intermediate or transition states are longer (slower) than subsequent kinetic steps, then the associated misfolding pathways may not be relevant under native, $\mathrm{K}^{+}$-containing conditions. 
Marchand and Gabelica describe a drastic acceleration of folding kinetics in the range of $<1 \mathrm{mM} \mathrm{K}^{+}$ (10 $\mu \mathrm{M}$ DNA). This effect seems to be mainly saturated in the range of $2-3 \mathrm{mM} \mathrm{K} \mathrm{K}^{+}(>100 \mu \mathrm{M}$ DNA). ${ }^{177}$

A rapid change of the ionic conditions is by no means physiological, and so is thermal denaturation. The approach presented in this thesis, using photocages to prepare unfolded states is a clear advancement in this regard, as it can be applied without further requirements to experimental and sample conditions. Folding can be studied under constant, isothermal experimental conditions with any biophysical detection method.

\subsubsection{Parallel Reaction Pathways Accelerate Folding (G-Register Isomers)}

This chapter relates to the major findings of the publication: "Parallel reaction pathways accelerate folding of a guanine quadruplex" (Harkness, Hennecker, Grün et al. NAR 2021). ${ }^{752}$ This publication contains contributions from me (the author of this thesis) using the photocaging approach for lightinduced folding.

In protein folding processes, the existence of parallel folding pathways reduces the entropy penalty for the folding, since the conformational entropy can be maximized. From these basic considerations ${ }^{753-759}$, the hypothesis follows that if different parallel folding pathways are occupied during G4 folding, the overall folding should be accelerated. The observable rate constant for the folding of a wildtype sequence into a structural ensemble that enfolds all conformations that can be possibly adopted $(\mathrm{x}, \mathrm{y}, \mathrm{z})$ is given as:

$$
\mathrm{k}_{\mathrm{F}, \mathrm{wt}}=\mathrm{k}_{\mathrm{F}, \mathrm{w}}+\mathrm{k}_{\mathrm{F}, \mathrm{x}}+\mathrm{k}_{\mathrm{F}, \mathrm{y}}+\mathrm{k}_{\mathrm{F}, \mathrm{z}}
$$

Given that the individual rate constants were equally fast and the related conformations were equally populated, folding of the wildtype would be $4 \mathrm{x}$ faster than the isolated conformations. Mittermaier and co-workers have outlined this hypothesis and investigated the predicted consequences on the four possible G-register isomer conformations from the 18 -mer $c M Y C-2345$ sequence $(33,35,55,53)$. Converting (1) into the following expression:

$$
\mathrm{k}_{\mathrm{F}, \mathrm{wt}} \cdot<\mathrm{k}_{\mathrm{F}, 33,35,53,55}>^{-1}=4
$$

(with $\left\langle\mathrm{k}_{\mathrm{F}, \ldots}\right\rangle$ as mean value of the rate constants) gives a folding acceleration that is equal to the number of folding pathways. They conducted a series of experiments based on rapid thermal (un)folding with thermal hysteresis, comparable to those presented in chapter 4.6.1. These experiments have been supported with light-induced (isothermal) folding experiments observed with real-time NMR (from me, the author of this thesis). The trend in these experiments supports the general idea that G4 folding can be significantly accelerated by occupation of multiple parallel folding pathways. 


\subsubsection{Enlarged Conformational Space Decelerates Folding (Spare-Tire Isomers)}

The influence of spare-tire isomers on the kinetics of the major folding pathways follows a different trend as observed for the G-register isomers. Here, the kinetics of the wildtype sequence with minimal restrictions to the conformational space are slower than those observed for the kinetics of the isolated major conformation. The wt-sequence is able to adopt all single isolated conformations and further all possible sub- and microstates, with unproductive interactions and misfolded species. This effect is expected for G4 folding with kinetic partitioning of competing basins of attraction on the folding energy landscape.

The fact that the competing minor conformations are also formed concurrently to the major conformation, despite their $\sim 4 \mathrm{x}$ slower folding kinetics, points towards irreversible folding steps that enable these slower pathways. These tipping points separate the folding pathways and lead to kinetic traps that collapse into the folding basins of the minor conformations. This results in an overall slower folding process (if described as a decay function of unfolded states towards any folded G4 structure.

\subsection{Folding intermediates}

In chapter 5.2.3 the experimental observation of a long-lived (several minutes at $285 \mathrm{~K}$ ) intermediate formation for $c M Y C-1234$ is presented. The kinetics for time-resolved NMR and CD spectra are superimposable and clearly indicate the formation of an anti-parallel conformation. Furthermore, the observation of eight well resolved imino proton resonances in the downfield-shifted region $(>12.1 \mathrm{ppm})$ point to eight G-residues in slow-exchange (base paired). Marchand and Gabelica ${ }^{167}$, as well as Gray et al. ${ }^{170}\left(298 \mathrm{~K}\right.$, up to $25 \mathrm{mM} \mathrm{K}^{+}$) have made strikingly similar observations on folding for cMYC-2345 (in 33 G-register conformation, PDB: 1XAV) with CD-spectroscopy. The cMYC-2345-33 G4 features the same loop geometry as the $c M Y C-1234$ G4 (lateral:proximal:lateral = 1:2:1 nts). They find anti-parallel intermediates persisting on a comparable time scale. Gray et al. have proposed a kinetic model that involves the formation of an anti-parallel chair G4 conformation that can evolve into the final all-parallel conformation. They suggest that this process does not require complete unfolding, because estimates for the unfolding rates (derived from complement trapping experiments) are significantly slower than the observed folding relaxation time. Finally, Marchand and Gabelica could show with mass spectrometry that the elusive anti-parallel intermediate binds only a single $\mathrm{K}^{+}$-ion.

The discussed experimental findings from the literature, in line with the experimental findings presented in this thesis, prompt proposing a 2-tetrad chair conformation as possible intermediate. Only the anti-parallel 2-tetrad G4 can account for all observed experimental data. 
The formation of this intermediate species is further supported by predictions from molecular dynamics. ${ }^{157,161-163}$ The formation of a 3-tetrad chair G4 as proposed by Gray et al. does not account (i) for the single occupied $\mathrm{K}^{+}$-binding site, and (ii) the resulting $1 \mathrm{nt}$ lateral loops are highly disfavoured for anti-parallel hairpins. The non-native syn/anti distribution in the chair G4 must be disentangled to enable a refolding transition to the all-anti sugar configuration in an all-parallel G4 conformation. Therefore, the observed intermediate formation is rather an off- than an on-pathway state.

The proposed structural model for the intermediate conformation is highly sensitive on the length of the proximal loop, which explains the differences in folding kinetics for the spare-tire isomers $c M Y C$ 2345 and 1245 (Figure 63). In the light-induced folding experiments for the cMYC-2345 in 53 G-register conformation (chapter 5.2.2), no stable intermediate formation was observed and folding was significantly faster compared to folding kinetics of other $c M Y C$ G4 conformations. The reason for this is likely the length of the $1 \mathrm{nt}$ proximal loop resulting in a 2:1:1 loop geometry that prevents a tetrad formation of initially collapses terminal hairpins.

The unparalleled increased internal flexibility in the resulting $6 \mathrm{nt}$ long proximal loop in the case of cMYC-1245, probably minimizes the chance for the formation of productive microstates. The separated hairpins are most likely not stable and will further collapse, but the coordination of $\mathrm{K}^{+}$and subsequent tetrad formation will be less effective in this compacted ensemble. This gives a reasonable explanation for the non-Arrhenius temperature dependence of $c M Y C-1245$ (5.3). This thermodynamic profile of folding kinetics is expected for hairpin folding. ${ }^{760-766}$ The propensity and stability of a stabilization in a G4-like arrangement, after the initial collapse into two intramolecular hairpins then is a rate-determining folding phase.

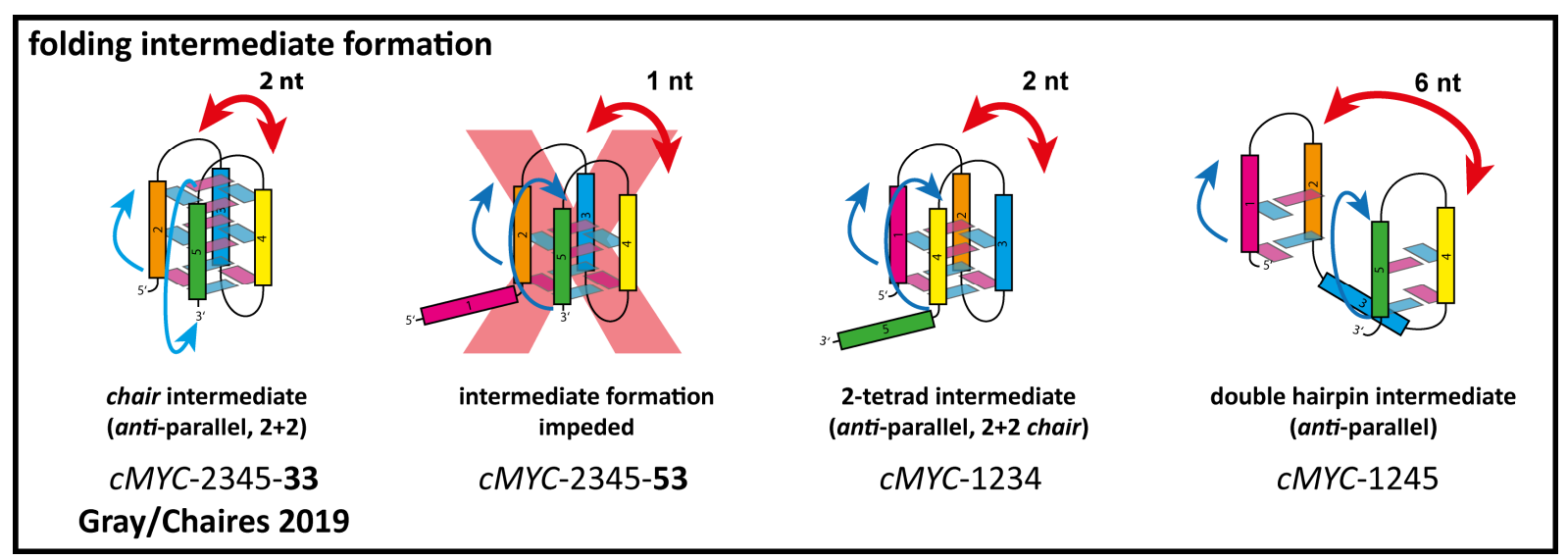

Figure 63: Different proposed folding intermediates and their stability in dependence to the length of the proximal loop. For $c M Y C-1234$ and $c M Y C-2345-33$, the 2 nt long proximal loop enables the intermediate formation of an anti-parallel chair G4. The resulting $1 \mathrm{nt}$ long proximal loop for $c M Y C-2345-53$ impedes the formation of defined tetrads in this strand arrangement. In case of $c M Y C-1245$, the increased flexibility in the $6 \mathrm{nt}$ long proximal loop separates the terminal hairpins (1-2 and 4-5) and thereby minimizes the probability of productive microstates and $\mathrm{K}^{+}$-coordination to form a 2 -tetrad G4. 


\subsection{Refolding Across Different Transitory Ensembles}

Refolding of folded G4 conformations has been investigated between spare-tire isomers (cMYC 1234 and 1245, transition to 2345) and different G-register isomers (between cMYC-2345-53 and cMYC-2345-33, both parallel; and between hTERT hybrid and parallel). The apparent activation energies and refolding kinetics allow proposing diverging mechanism that require different degrees of unfolding in the transitory ensemble. The experimental data can be explained following a simplified depiction of strand rearrangements as a reasonable model. As discussed above, the experimental data support the assumption that the observed apparent activation energies reflect the same rate-limiting step in all investigated refolding processes (chapters 4.5, 4.6.1 and 5.4.2). The subsequent refolding kinetics however are remarkably different, which links to different rearrangement steps.

Formally, the G-register exchange process for the $c M Y C$ isomers and the spare-tire exchange process for the transition from $c M Y C 1234$ to 2345 requires only the removal of one G-tract, followed by the re-incorporation of the same, shifted G-tract; or the incorporation of another G-tract as substitute. The kinetic observations for both processes are strikingly similar, which prompts the proposal of a triplex-like strand orientation. For the refolding of the hTERT G-register isomers as well as for refolding of the $c M Y C 1245$ spare-tire isomer, this degree of unfolding is not sufficient for the transition between these conformations. Both pairs of G4 isomers require at least a reorientation of two G-tracts, $h T E R T$ in addition requires redistribution of syn/anti configurations.
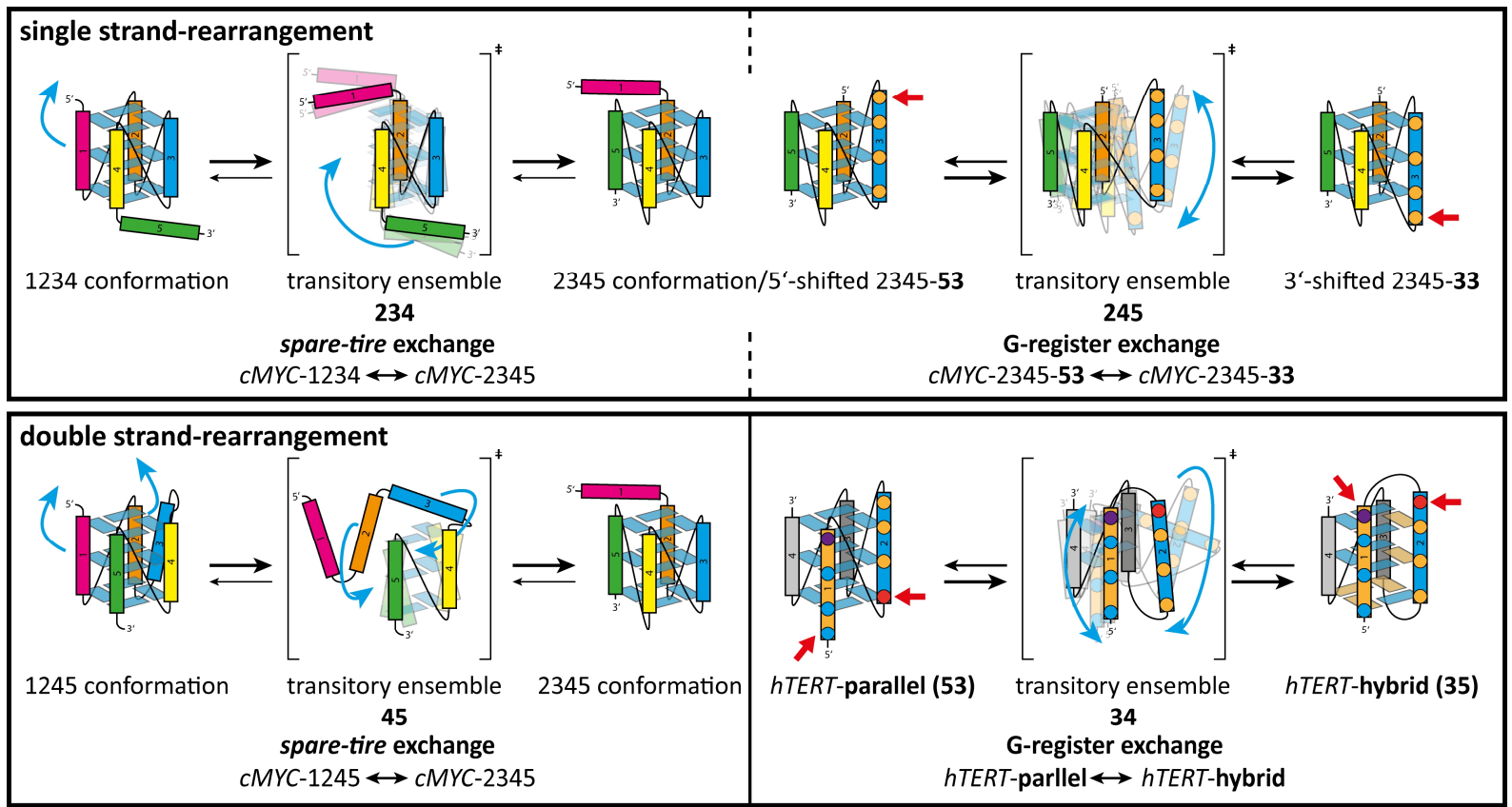

Figure 64: Different proposed species in the transitory ensembles for spare-tire exchange and G-register exchange dynamics. The measured refolding kinetics and analysis of the apparent activation energies support the hypothesis that the refolding mechanisms require different degrees of unfolding. A single strand-rearrangement does not necessarily require a complete unfolding-folding transition, but can evolve through compacted ensembles. 
The proposed model for the different transitory ensembles is based on the experimental findings within this thesis. It is further supported by predictions from molecular dynamics simulations, on comparable systems. ${ }^{157,161-164}$ However, it cannot possibly account for putatively involved short-lived microstates that emerge from conformational diffusion on the sub- $\mu$ s timescale. The real polymer physics of the underlying mechanisms will be certainly more complex.

\subsection{Non-Canonical Structural Polymorphism as Challenge}

DNA G-quadruplexes are evasive and volatile structural species with an intrinsic dynamic nature. Within this thesis, the conformational dynamics related to the non-canonical G4-structural features of spare-tire and G-register isomerism have been described in the $c M Y C$ promoter G4 ensemble. The literature gives strong indications that these $c M Y C-G 4$ dynamics and the polymorphic character of the NHE-III ${ }_{1}$ are vital and integral components for their functionality in gene regulation. ${ }^{99,340,341,440}$ The disentanglement of G4 ensembles into distinct folded conformations is however crucial to understand their structure-function relationship and the mechanisms that drive the dynamics and thermodynamic properties within such somehow blurred structural ensembles. Even small changes in the experimental conditions or the primary sequences of G4s have a drastic effect on the structural integrity. ${ }^{767}$

In this regard, the herein developed and presented strategy that utilizes photocages for conformational selection or suppression represents tremendous advantages over hitherto reported methods to investigate G4 folding (2.2.2). The approach was successful to trap and isolated different folded or unfolded states in all presented cases. However, Figure 48 in chapter 4.6 .2 (triple caged cMYC-2345-X3 18-mer), shows that the effective inhibition of base pair interactions remains challenging and is not per se straightforward. The photocaging strategy with a (close to) minimal use of photocages has to be adjusted to a given oligonucleotide sequence to prevent pre- or partially folded species.

Chapter 4.2 as well as many examples from the literature ${ }^{144,159,160,172}$ show the challenge to accomplish a complete unfolding in the absence of $\mathrm{K}^{+}$, besides the additional considerations that might bias investigation of structural dynamics with altered ionic conditions (chapters 2.2.2,6.1.1). The presence of pre-folded structures is a crucial aspect that has tremendous effects on the folding pathways and the resulting folded conformations. ${ }^{157-160,167}$ The thermodynamic effects that arise from sequence modifications to tailor single conformations are also not negligible. This can be seen e.g. for a direct comparison of the effects of G-to-T vs. G-to-A or G-to-I mutations in the same oligonucleotide sequence $\left(c M Y C-G 4^{101,460,461}\right)$.

The successful application of transient chemical modification to G4 forming oligonucleotides with photocages is therefore remarkable. The time-resolved relaxation processes that have been presented 
can be considered mostly unbiased by any of the discussed external or intrinsic factors (ionic conditions, temperature, sequence modifications, conformational entropy) that potentially disturb the folding energy landscape. Restraints to the investigated sequences (with respect to the respective wt) or e.g. buffer conditions ( $\left[\mathrm{K}^{+}\right]$below physiological concentrations) have been deliberately introduced. The methodological approach however is universally applicable to diverse oligonucleotide sequences and experimental or sample conditions.

\subsection{Model for cMYC G4 (Re)-Folding including Non-Canonical Polymorphism}

The experimental data presented in this thesis allow drawing a comprehensive picture of the conformational landscape of the full-length G4 forming sequence found in the NHE-III 1 of the $c M Y C$ promoter. (Figure 65). The presented model includes the possible formation of both types of noncanonical polymorphism, which is G-register (chapter 4) and spare-tire (chapter 5) isomers. The major folding pathways have been delineated, important intermediate and meta-stable states have been characterized and key transitory ensembles are proposed.

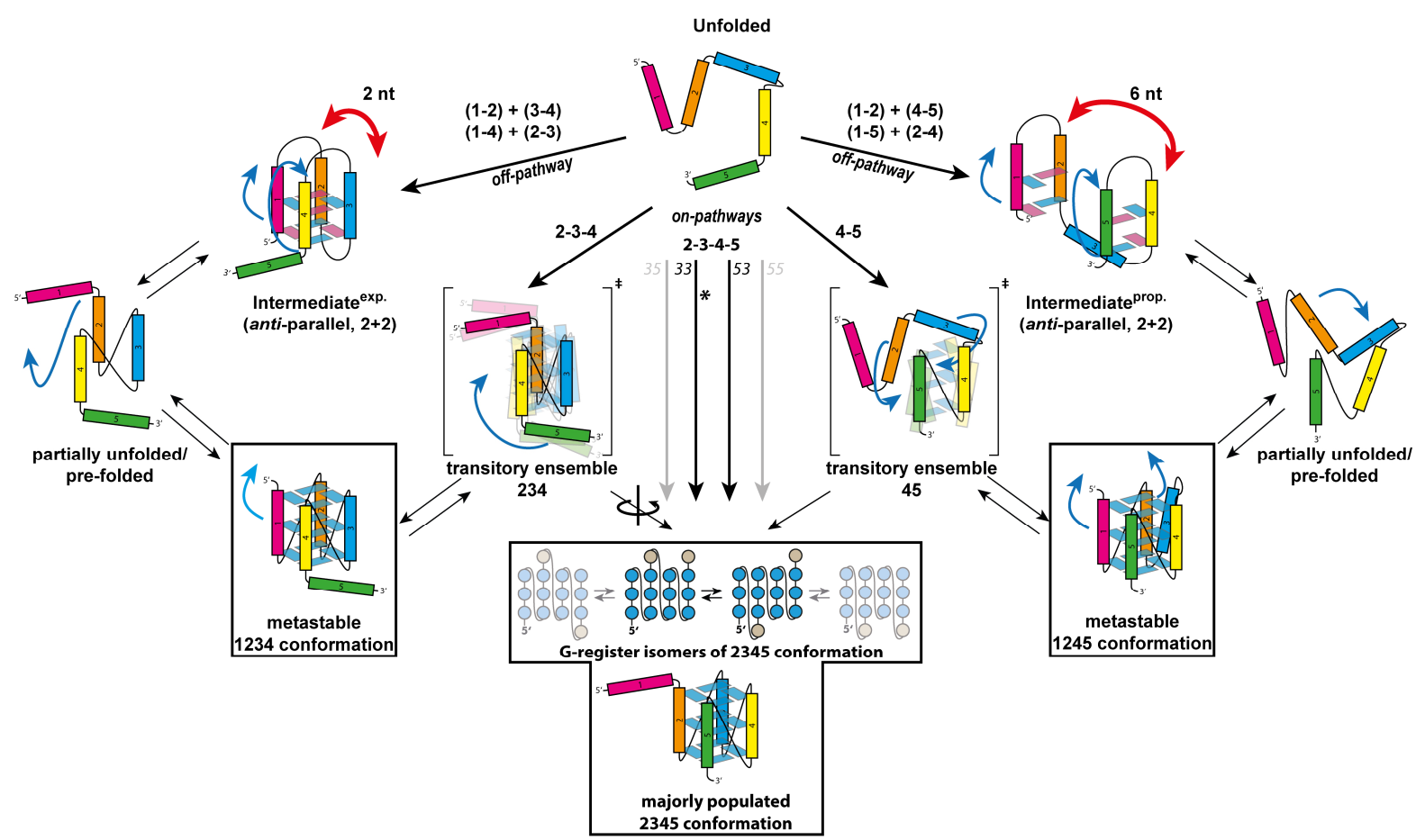

Figure 65: Overview and model for the major folding and refolding pathways in the $c M Y C$ NHE-III 1 promoter G4. The model is based on experimental findings presented in this thesis, experimental data from the literature and the abovediscussed general considerations. The depicted model accounts for non-canonical structural polymorphism, including spare-tire and G-register isomers. ( ${ }^{*}$ see additional intermediate formation for cMYC-2345-33 in Figure 63. Folding pathway/mechanism reported by Gray et al. ${ }^{170}$ ).

One of the key findings is that all different folding pathways can be occupied, starting from an unfolded state. The major on- and off-pathways here are defined with regard to the major populated 2345 conformations, including its G-register isomers. This definition might be misleading, since the major 
off-pathways lead to the minor conformations 1234 and 1245, which themselves are no misfolded states, but rather alternative conformations.

However, the minor conformations are thermodynamically less stable, leading to refolding towards the major conformation at ambient temperatures. The on- and off-pathways have different impact on the overall folding kinetics: while a significant population of parallel off-pathways causes a deceleration, the parallel on-pathways cause an accelerated folding.

To go along these pathways, the early-stage, initial hydrogen bond interactions and orientation of the oligonucleotide backbone must be decisive. Otherwise, these pathways would not be productive by any means, since the kinetics of the pure on-pathways are significantly faster. This means that the initial, reversible dynamics (pre-equilibrium) of these pre-folded contacts must be kinetically in the same time range as subsequent steps or slightly slower. The on-pathways therefore lead to accelerated folding, since here more contacts between residues that are part of the major conformation may lead to productive macrostates. The irreversible decision (or branching) for any of the four possible G-register isomers might be on a late-stage along the folding pathway. This means that after initial pre-formation (including G-tracts 2-3-4-5) four different isomers share a common route along the folding energy landscape, leading to an acceleration of overall folding. Only in this scenario, the enlarged conformational entropy has this kinetic effect.

\subsection{Conclusion and Outlook}

Within the scope of this thesis important conformational dynamics related to newly discovered noncanonical structural features of DNA G4s have been described, as discussed and summarized in chapter 6. The presented experimental approach that exploits a photocaging approach for conformational selection and conformational suppression is a major improvement of hitherto reported experimental approaches to measure G4 kinetics. The experimental findings and their presented analysis have shed light on the dynamic features of the polymorphic G4 ensemble in the $\mathrm{NHE}^{-\mathrm{III}_{1}}$ of the $c M Y C$ promoter. This insight will further deepen the understanding of the pivotal biophysical properties that govern G4 functionality.

In upcoming investigations, it will be highly desirable to elucidate the influence of $c M Y C$ G4 interacting proteins. Evidence is growing within the literature, that the binding affinities and processing efficiencies of binding proteins and helicases are sensitive to the described conformations with noncanonical polymorphism. Further, the rather slow interconversion dynamics harbour the speculation that there might exist proteins with a refolding chaperoning activity in the cell. Newly developed NMR methods (such as ultrafast $2 \mathrm{D}-\mathrm{NMR}^{634}$ in combination with signal enhancement ${ }^{639,641,768}$ ) will help to expand the experimental toolkit to tackle this challenge. 


\section{References}

\section{References}

1. Dobson, C. M. Protein folding and misfolding. Nature 426, 884-890 (2003).

2. Vendruscolo, M., Zurdo, J., MacPhee, C. E. \& Dobson, C. M. Protein folding and misfolding: a paradigm of self-assembly and regulation in complex biological systems. Philos. Trans. R. Soc. London. Ser. A Math. Phys. Eng. Sci. 361, 1205-1222 (2003). Dawkins, R. River Out Of Eden: A Darwinian View of Life. (page 133, Basic Books/Weidenfeld\&Nicolson, ISBN: 0-297-81540-7, 1995). Dahm, R. Friedrich Miescher and the discovery of DNA. Developmental Biology (2005). doi:10.1016/j.ydbio.2004.11.028 Dahm, R. Discovering DNA: Friedrich Miescher and the early years of nucleic acid research. Human Genetics 122, 565-581 (2008). Chargaff, E., Lipshitz, R. \& Green, C. Composition of the desoxypentose nucleic acids of four genera of sea-urchin. J. Biol. Chem. 195, 155-60 (1952).

7. Watson, J. D. \& Crick, F. H. C. Molecular Structure of Nucleic Acids: A Structure for Deoxyribose Nucleic Acid. Nature 171, 737-738 (1953)

8. Wilkins, M. H. F., Stokes, A. R. \& Wilson, H. R. Molecular Structure of Nucleic Acids: Molecular Structure of Deoxypentose Nucleic Acids. Nature 171, 738-740 (1953).

9. Franklin, R. E. \& Gosling, R. G. Molecular Configuration in Sodium Thymonucleate. Nature 171, 740-741 (1953).

10. Franklin, R. E. \& Gosling, R. G. The structure of sodium thymonucleate fibres. I. The influence of water content. Acta Crystallogr. (1953). doi: $10.1107 / \mathrm{s} 0365110 \times 53001939$

11. Marvin, D. A., Spencer, M., Wilkins, M. H. F. \& Hamilton, L. D. The molecular configuration of deoxyribonucleic acid III. X-ray diffraction study of the C form of the lithium salt. J. Mol. Biol. 3, 547-IN14 (1961).

12. Mitsui, Y. et al. Physical and Enzymatic Studies on Poly d(I-C).Poly d(I-C), an Unusual Double-helical DNA. Nature 228, 1166-1169 (1970). Wang, A. H. J. et al. Molecular structure of a left-handed double helical DNA fragment at atomic resolution. Nature 282, 680-686 (1979). Wacker, A. et al. Secondary structure determination of conserved SARS-CoV-2 RNA elements by NMR spectroscopy. Nucleic Acids Res. 48, 12415-12435 (2020).

15. Wacker, A. et al. Structure and dynamics of the deoxyguanosine-sensing riboswitch studied by NMR-spectroscopy. Nucleic Acids Res. 39, 68026812 (2011). Niehrs, C. \& Luke, B. Regulatory R-loops as facilitators of gene expression and genome stability. Nat. Rev. Mol. Cell Biol. 21, 167-178 (2020). Nasiri, A. H., Wurm, J. P., Immer, C., Weickhmann, A. K. \& Wöhnert, J. An intermolecular G-quadruplex as the basis for GTP recognition in the class V-GTP aptamer. RNA 22, 1750-1759 (2016).

18. mmer, C., Hacker, C. \& Wöhnert, J. Solution structure and RNA-binding of a minimal ProQ-homolog from Legionella pneumophila (Lpp1663). RNA 26, 2031-2043 (2020).

19. Ellington, A. D. \& Szostak, J. W. In vitro selection of RNA molecules that bind specific ligands. Nature 346, 818-822 (1990).

20. Tuerk, C. \& Gold, L. Systematic evolution of ligands by exponential enrichment: RNA ligands to bacteriophage T4 DNA polymerase. Science 249, 505-510 (1990).

21. Ellington, A. D. \& Szostak, J. W. Selection in vitro of single-stranded DNA molecules that fold into specific ligand-binding structures. Nature 355, 850-852 (1992).

22. Green, S. J., Lubrich, D. \& Turberfield, A. J. DNA hairpins: Fuel for autonomous DNA devices. Biophys. J. (2006). doi:10.1529/biophysj.106.084681

23. Breaker, R. R. DNA enzymes. Nat. Biotechnol. 15, 427-431 (1997).

24. Silverman, S. K. DNA as a Versatile Chemical Component for Catalysis, Encoding, and Stereocontrol. Angew. Chem. Int. Ed. 49, 7180-7201 (2010).

25. Ponce-Salvatierra, A., Wawrzyniak-Turek, K., Steuerwald, U., Höbartner, C. \& Pena, V. Crystal structure of a DNA catalyst. Nature 529, 231234 (2016).

26. Wirmer-Bartoschek, J. \& Schwalbe, H. Understanding How DNA Enzymes Work. Angew. Chem. Int. Ed. 55, 5376-5377 (2016).

27. Stojanovic, M. N., de Prada, P. \& Landry, D. W. Aptamer-Based Folding Fluorescent Sensor for Cocaine. J. Am. Chem. Soc. 123, 4928-4931 (2001).

Bock, L. C., Griffin, L. C., Latham, J. A., Vermaas, E. H. \& Toole, J. J. Selection of single-stranded DNA molecules that bind and inhibit human thrombin. Nature (1992). doi:10.1038/355564a0

Heckel, A. \& Mayer, G. Light Regulation of Aptamer Activity: An Anti-Thrombin Aptamer with Caged Thymidine Nucleobases. J. Am. Chem. Soc. 127, 822-823 (2005).

Thiviyanathan, V. et al. Hybrid-hybrid matrix structural refinement of a DNA three-way junction from 3D NOESY-NOESY. J. Biomol. NMR 14, 209-21 (1999).

31. van Buuren, B. N. ., Overmars, F. J. J., Ippel, J. H., Altona, C. \& Wijmenga, S. S. Solution structure of a DNA three-way junction containing two unpaired thymidine bases. identification of sequence features that decide conformer selection. J. Mol. Biol. 304, 371-383 (2000).

32. Duckett, D. R. \& Lilley, D. M. The three-way DNA junction is a Y-shaped molecule in which there is no helix-helix stacking. EMBO J. 9, 16591664 (1990).

33. Assenberg, R. Sequence-dependent folding of DNA three-way junctions. Nucleic Acids Res. 30, 5142-5150 (2002).

34. Liu, Y. \& West, S. C. Happy Hollidays: 40th anniversary of the Holliday junction. Nat. Rev. Mol. Cell Biol. 5, 937-944 (2004).

35. Holliday, R. A mechanism for gene conversion in fungi. Genet. Res. 5, 282-304 (1964).

36. Haider, S. et al. Holliday Junctions Formed from Human Telomeric DNA. J. Am. Chem. Soc. 140, 15366-15374 (2018).

37.

Shiba, T., Iwasaki, H., Nakata, A. \& Shinagawa, H. SOS-inducible DNA repair proteins, RuvA and RuvB, of Escherichia coli: functional interactions between RuvA and RuvB for ATP hydrolysis and renaturation of the cruciform structure in supercoiled DNA. Proc. Natl. Acad. Sci. 88, 8445-8449 (1991).

38. Shlyakhtenko, L. S., Potaman, V. N., Sinden, R. R. \& Lyubchenko, Y. L. Structure and dynamics of supercoil-stabilized DNA cruciforms. J. Mol. Biol. 280, 61-72 (1998).

39. Schroth, G. P. \& Ho, P. S. Occurrence of potential cruciform and H-DNA forming sequences in genomic DNA. Nucleic Acids Res. 23, 19771983 (1995).

40. Brázda, V., Laister, R. C., Jagelská, E. B. \& Arrowsmith, C. Cruciform structures are a common DNA feature important for regulating biological processes. BMC Mol. Biol. 12, 33 (2011).

41. Zazopoulos, E., Lalli, E., Stocco, D. M. \& Sassone-Corsi, P. DNA binding and transcriptional repression by DAX-1 blocks steroidogenesis. Nature 390, 311-315 (1997). 


\section{References}

42.

43.

44.

45

47.

48.

49.

50 .

51.

52.

53.

54.

55

56.

57.

58.

59.

60.

61.

62.

63.

64.

65.

66.

67.

68.

69.

Bikard, D., Loot, C., Baharoglu, Z. \& Mazel, D. Folded DNA in Action: Hairpin Formation and Biological Functions in Prokaryotes. Microbiol. Mol. Biol. Rev. 74, 570-588 (2010).

Greider, C. W. Telomeres Do D-Loop-T-Loop Minireview. Cell (1999).

Cornish-Bowden, A. Abbreviations and symbols for the description of conformations of polynucleotide chains (Recommendations 1982). Pure Appl. Chem. 55, 1273-1280 (1983).

Markley, J. L. et al. Recommendations for the presentation of NMR structures of proteins and nucleic acids. IUPAC-IUBMB-IUPAB InterUnion Task Group on the Standardization of Data Bases of Protein and Nucleic Acid Structures Determined by NMR Spectroscopy. J. Biomol. NMR 12, 1-23 (1998).

Mirkin, S. M. Discovery of alternative DNA structures: a heroic decade (1979-1989). Front. Biosci. 13, 1064-71 (2008).

Shing, P. \& Carter, M. DNA Structure: Alphabet Soup for the Cellular Soul. in DNA Replication-Current Advances (InTech, 2011). doi: $10.5772 / 18536$

Hoogsteen, K. The crystal and molecular structure of a hydrogen-bonded complex between 1-methylthymine and 9-methyladenine. Acta Crystallogr. 16, 907-916 (1963).

Nikolova, E. N. et al. Transient Hoogsteen base pairs in canonical duplex DNA. Nature 470, 498-502 (2011).

Watson, J. D. \& Crick, F. H. C. The Structure of DNA. Cold Spring Harb. Symp. Quant. Biol. 18, 123-131 (1953).

Yagil, G. Paranemic Structures of DNA and their Role in DNA Unwinding. Crit. Rev. Biochem. Mol. Biol. 26, 475-559 (1991).

van Dongen, M. J. et al. Structure and mechanism of formation of the H-y5 isomer of an intramolecular DNA triple helix. Nat. Struct. Biol. 6, 854-9 (1999).

Pestov, D. G., Dayn, A., Siyanova EYu, George, D. L. \& Mirkin, S. M. H-DNA and Z-DNA in the mouse c-Ki-ras promoter. Nucleic Acids Res. 19, 6527-32 (1991).

Kinniburgh, A. J. A cis-acting transcription element of the c-myc gene can assume an H-DNA conformation. Nucleic Acids Res. 17, 7771-8 (1989).

Radhakrishnan, I. \& Patel, D. J. Solution structure of a purine.purine-pyrimidine DNA triplex containing G.GC and T.AT triples. Structure 1, 135-152 (1993)

Radhakrishnan, I. \& Patel, D. J. Solution structure of a pyrimidine-purine-pyrimidine DNA triplex containing T·AT, C+ $\cdot G C$ and G.TA triples. Structure 2, 17-32 (1994).

Pandey, S. et al. Transcription blockage by stable H-DNA analogs in vitro. Nucleic Acids Res. 43, 6994-7004 (2015).

Christensen, J. J., Wrathall, D. P., Izatt, R. M. \& Tolman, D. O. Thermodynamics of proton dissociation in dilute aqueous solution. IX. pK, changes in macroenthalpy, and changes in macroentropy values for proton ionization from o-, $\mathrm{m}-$, and $\mathrm{p}$-aminobenzoic acids and their methyl esters at 25 degrees. J. Phys. Chem. 71, 3001-6 (1967).

Gellert, M., Lipsett, M. N. \& Davies, D. R. Helix Formation by Guanylic Acid. Proc. Natl. Acad. Sci. 48, 2013-2018 (1962).

Sen, D. \& Gilbert, W. Formation of parallel four-stranded complexes by guanine-rich motifs in DNA and its implications for meiosis. Nature 334, 364-366 (1988).

Sen, D. \& Gilbert, W. A sodium-potassium switch in the formation of four-stranded G4-DNA. Nature 344, 410-4 (1990).

Detellier, C. \& Laszlo, P. Role of alkali metal and ammonium cations in the self-assembly of the 5'-guanosine monophosphate dianion. J. Am. Chem. Soc. 102, 1135-1141 (1980).

Borzo, M., Detellier, C., Laszlo, P. \& Paris, A. Proton, sodium-23, and phosphorus-31 NMR studies of the self-assembly of the 5'-guanosine monophosphate dianion in neutral aqueous solution in the presence of sodium cations. J. Am. Chem. Soc. 102, 1124-1134 (1980).

Hud, N. V. \& Plavec, J. The Role of Cations in Determining Quadruplex Structure and Stability. in Quadruplex Nucleic Acids (eds. Neidle, S. \& Balasubramanian, S.) 100-130 (2007). doi:10.1039/9781847555298-00100

Largy, E., Mergny, J.-L. \& Gabelica, V. Role of Alkali Metal Ions in G-Quadruplex Nucleic Acid Structure and Stability. in Metal Ions in Life Sciences 203-258 (2016). doi:10.1007/978-3-319-21756-7_7

Biffi, G., Tannahill, D., McCafferty, J. \& Balasubramanian, S. Quantitative visualization of DNA G-quadruplex structures in human cells. Nat. Chem. 5, 182-186 (2013).

Zhang, S. et al. Real-time monitoring of DNA G-quadruplexes in living cells with a small-molecule fluorescent probe. Nucleic Acids Res. 46, $7522-7532$ (2018).

Di Antonio, M. et al. Single-molecule visualization of DNA G-quadruplex formation in live cells. Nat. Chem. 12, $832-837$ (2020). in the cytoplasm of human cells. Nat. Chem. 6, 75-80 (2014).

Laguerre, A. et al. Visualization of RNA-Quadruplexes in Live Cells. J. Am. Chem. Soc. 137, 8521-8525 (2015).

Liu, H.-H. et al. RNA G-quadruplex formation in defined sequence in living cells detected by bimolecular fluorescence complementation. Chem. Sci. 7, 4573-4581 (2016).

Guo, J. U. \& Bartel, D. P. RNA G-quadruplexes are globally unfolded in eukaryotic cells and depleted in bacteria. Science 353, aaf5371-aaf5371 (2016).

Gehring, K., Leroy, J. L. \& Guéron, M. A tetrameric DNA structure with protonated cytosine.cytosine base pairs. Nature 363, 561-565 (1993). Guéron, M. \& Leroy, J. L. The i-motif in nucleic acids. Current Opinion in Structural Biology 10, 326-331 (2000).

Esmaili, N. \& Leroy, J. L. i-motif solution structure and dynamics of the d(AACCCC) and d(CCCCAA) tetrahymena telomeric repeats. Nucleic Acids Res. 33, 213-24 (2005).

6. Lieblein, A. L., Buck, J., Schlepckow, K., Fürtig, B. \& Schwalbe, H. Time-Resolved NMR Spectroscopic Studies of DNA i-Motif Folding Reveal Kinetic Partitioning. Angew. Chem. Int. Ed. 51, 250-253 (2012).

Lieblein, A. L., Krämer, M., Dreuw, A., Fürtig, B. \& Schwalbe, H. The Nature of Hydrogen Bonds in Cytidine $\cdots$ H $+\cdots$ Cytidine DNA Base Pairs. Angew. Chem. Int. Ed. 51, 4067-4070 (2012).

Lieblein, A. L., Fürtig, B. \& Schwalbe, H. Optimizing the Kinetics and Thermodynamics of DNA i-Motif Folding. ChemBioChem 14, 12261230 (2013).

Day, H. A., Pavlou, P. \& Waller, Z. A. E. I-Motif DNA: Structure, stability and targeting with ligands. Bioorganic Med. Chem. 22, 4407-4418 (2014).

Lannes, L., Halder, S., Krishnan, Y. \& Schwalbe, H. Tuning the pH Response of i-Motif DNA Oligonucleotides. ChemBioChem 16, 1647-1656 (2015).

Lannes, L., Young, P., Richter, C., Morgner, N. \& Schwalbe, H. Interaction of the N-Terminal Tandem Domains of hnRNP LL with the BCL2 Promoter i-Motif DNA Sequence. ChemBioChem 18, 2033-2044 (2017). 


\section{References}

Wright, E. P., Huppert, J. L. \& Waller, Z. A. E. Identification of multiple genomic DNA sequences which form i-motif structures at neutral pH. Nucleic Acids Res. 45, 2951-2959 (2017).

Abou Assi, H., Garavís, M., González, C. \& Damha, M. J. i-Motif DNA: structural features and significance to cell biology. Nucleic Acids Res. 46, 8038-8056 (2018).

Zeraati, M. et al. I-motif DNA structures are formed in the nuclei of human cells. Nat. Chem. 10, 631-637 (2018).

Agrawal, P., Hatzakis, E., Guo, K., Carver, M. \& Yang, D. Solution structure of the major G-quadruplex formed in the human VEGF promoter in K+: Insights into loop interactions of the parallel G-quadruplexes. Nucleic Acids Res. 41, 10584-10592 (2013).

Bochman, M. L., Paeschke, K. \& Zakian, V. A. DNA secondary structures: stability and function of G-quadruplex structures. Nat. Rev. Genet. 13, 770-780 (2012).

Dvorkin, S. A., Karsisiotis, A. I. \& Webba da Silva, M. Encoding canonical DNA quadruplex structure. Sci. Adv. 4, eaat3007 (2018).

Maity, A., Winnerdy, F. R., Chang, W. D., Chen, G. \& Phan, A. T. Intra-locked G-quadruplex structures formed by irregular DNA G-rich motifs. Nucleic Acids Res. 48, 3315-3327 (2020).

Cui, X. et al. Exploration of the Structure and Recognition of a G-quadruplex in the her2 Proto-oncogene Promoter and Its Transcriptional Regulation. Sci. Rep. 9, 3966 (2019).

Palumbo, S. L. et al. A novel G-quadruplex-forming GGA repeat region in the c-myb promoter is a critical regulator of promoter activity. Nucleic Acids Res. 36, 1755-1769 (2008).

Adrian, M. et al. Structure and Conformational Dynamics of a Stacked Dimeric G-Quadruplex Formed by the Human CEB1 Minisatellite. J. Am. Chem. Soc. 136, 6297-6305 (2014).

Macaya, R. F., Schultze, P., Smith, F. W., Roe, J. A. \& Feigon, J. Thrombin-binding DNA aptamer forms a unimolecular quadruplex structure in solution. Proc. Natl. Acad. Sci. 90, 3745-3749 (1993).

Podbevsek, P., Hud, N. V. \& Plavec, J. NMR evaluation of ammonium ion movement within a unimolecular G-quadruplex in solution. Nucleic Acids Res. 35, 2554-2563 (2007).

Brcic, J. \& Plavec, J. NMR structure of a G-quadruplex formed by four d(G4C2) repeats: insights into structural polymorphism. Nucleic Acids Res. 46, 11605-11617 (2018).

Lightfoot, H. L., Hagen, T., Tatum, N. J. \& Hall, J. The diverse structural landscape of quadruplexes. FEBS Lett. 593, 2083-2102 (2019).

Piazza, A. et al. Non-Canonical G-quadruplexes cause the hCEB1 minisatellite instability in Saccharomyces cerevisiae. Elife 6, (2017).

Varizhuk, A. et al. The expanding repertoire of G4 DNA structures. Biochimie 135, 54-62 (2017).

Patel, D. J., Phan, A. T. \& Kuryavyi, V. Human telomere, oncogenic promoter and 5'-UTR G-quadruplexes: diverse higher order DNA and RNA targets for cancer therapeutics. Nucleic Acids Res. 35, 7429-7455 (2007).

Seenisamy, J. et al. The Dynamic Character of the G-Quadruplex Element in the c-MYC Promoter and Modification by TMPyP4. J. Am. Chem. Soc. 126, 8702-8709 (2004).

Hatzakis, E., Okamoto, K. \& Yang, D. Thermodynamic Stability and Folding Kinetics of the Major G-Quadruplex and Its Loop Isomers Formed in the Nuclease Hypersensitive Element in the Human c-Myc Promoter: Effect of Loops and Flanking Segments on the Stability of Parallel-Stranded Intramolecul. Biochemistry 49, 9152-9160 (2010).

Harkness, R. W. \& Mittermaier, A. K. G-register exchange dynamics in guanine quadruplexes. Nucleic Acids Res. 44, 3481-3494 (2016).

Lim, K. W. et al. Coexistence of Two Distinct G-Quadruplex Conformations in the hTERT Promoter. J. Am. Chem. Soc. 132, 12331-12342 (2010).

Monsen, R. C. et al. The hTERT core promoter forms three parallel G-quadruplexes. Nucleic Acids Res. 48, 5720-5734 (2020).

Cheung, I., Schertzer, M., Rose, A. \& Lansdorp, P. M. Disruption of dog-1 in Caenorhabditis elegans triggers deletions upstream of guaninerich DNA. Nat. Genet. 31, 405-409 (2002).

Zhao, Y., O’Neil, N. J. \& Rose, A. M. Poly-G/poly-C tracts in the genomes of Caenorhabditis. BMC Genomics 8, 403 (2007).

Sengar, A., Heddi, B. \& Phan, A. T. Formation of G-Quadruplexes in Poly-G
Quadruplex Formed by a G 15 Stretch. Biochemistry 53, 7718-7723 (2014).

Quadruplex Formed by a G 15 Stretch. Biochemistry 53, 7718-7723 (2014).

Li, X. et al. Guanine-vacancy-bearing G-quadruplexes responsive to guanine derivatives. Proc. Natl. Acad. Sci. U. S. A. 112, 14581-6 (2015).

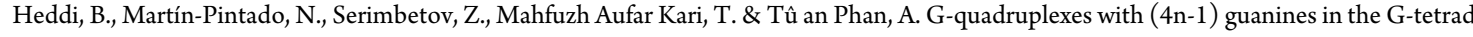
core: formation of a G-triad-water complex and implication for small-molecule binding. Nucleic Acids Res. 44, 910-916 (2016).

Wang, K.-B., Dickerhoff, J., Wu, G. \& Yang, D. PDGFR- $\beta$ Promoter Forms a Vacancy G-Quadruplex that Can Be Filled in by dGMP: Solution Structure and Molecular Recognition of Guanine Metabolites and Drugs. J. Am. Chem. Soc. 142, 5204-5211 (2020).

He, Y. et al. Selective Targeting of Guanine-Vacancy-Bearing G-Quadruplexes by G-Quartet Complementation and Stabilization with a Guanine-Peptide Conjugate. J. Am. Chem. Soc. 142, 11394-11403 (2020).

Phan, A. T., Modi, Y. S. \& Patel, D. J. Propeller-Type Parallel-Stranded G-Quadruplexes in the Human c-myc Promoter. J. Am. Chem. Soc. 126, $8710-8716(2004)$.

Jana, J. \& Weisz, K. A Thermodynamic Perspective on Potential G-Quadruplex Structures as Silencer Elements in the MYC Promoter. Chem. - A Eur. J. 26, 17242-17251 (2020).

3. Fleming, A. M., Zhou, J., Wallace, S. S. \& Burrows, C. J. A Role for the Fifth G-Track in G-Quadruplex Forming Oncogene Promoter Sequences during Oxidative Stress: Do These "Spare Tires" Have an Evolved Function? ACS Cent. Sci. 1, 226-233 (2015).

Do, N. Q. \& Phan, A. T. Monomer-Dimer Equilibrium for the 5'-5' Stacking of Propeller-Type Parallel-Stranded G-Quadruplexes: NMR Structural Study. Chem. Eur. J. 18, 14752-14759 (2012).

5. Palumbo, S. L., Ebbinghaus, S. W. \& Hurley, L. H. Formation of a Unique End-to-End Stacked Pair of G-Quadruplexes in the hTERT Core Promoter with Implications for Inhibition of Telomerase by G-Quadruplex-Interactive Ligands. J. Am. Chem. Soc. 131, 10878-10891 (2009). Chaires, J. B. et al. An Improved Model for the hTERT Promoter Quadruplex. PLoS One 9, e115580 (2014).

7. Song, J. H. et al. Small-Molecule-Targeting Hairpin Loop of hTERT Promoter G-Quadruplex Induces Cancer Cell Death. Cell Chem. Biol. 26, 1110-1121.e4 (2019).

18. Smargiasso, N. et al. G-quadruplex DNA assemblies: loop length, cation identity, and multimer formation. J. Am. Chem. Soc. 130, 10208-16 (2008).

19. Marsh, T. C., Vesenka, J. \& Henderson, E. A new DNA nanostructure, the G-wire, imaged by scanning probe microscopy. Nucleic Acids Res. 23, 696-700 (1995).

Marsh, T. C. \& Henderson, E. G-Wires: Self-Assembly of a Telomeric Oligonucleotide, d(GGGGTTGGGG), into Large Superstructures. Biochemistry 33, 10718-10724 (1994). 


\section{References}

121. Bose, K., Lech, C. J., Heddi, B. \& Phan, A. T. High-resolution AFM structure of DNA G-wires in aqueous solution. Nat. Commun. 9, 1959 (2018).

122. Mukundan, V. T. \& Phan, A. T. Bulges in G-quadruplexes: Broadening the definition of G-quadruplex-forming sequences. J. Am. Chem. Soc. 135, 5017-5028 (2013).

123. Meier, M. et al. Structure and hydrodynamics of a DNA G-quadruplex with a cytosine bulge. Nucleic Acids Res. 46, 5319-5331 (2018).

124. Lenarčič Živković, M., Rozman, J. \& Plavec, J. Structure of a DNA G-Quadruplex Related to Osteoporosis with a G-A Bulge Forming a Pseudoloop. Molecules 25, 4867 (2020).

125. Das, P. et al. Bulges in left-handed G-quadruplexes. Nucleic Acids Res. 49, 1724-1736 (2021).

126. Onel, B. et al. A New G-Quadruplex with Hairpin Loop Immediately Upstream of the Human BCL2 P1 Promoter Modulates Transcription. J. Am. Chem. Soc. 138, 2563-2570 (2016).

127. Ngoc Nguyen, T. Q., Lim, K. W. \& Phan, A. T. Duplex formation in a G-quadruplex bulge. Nucleic Acids Res. 48, 10567-10575 (2020).

128. Phan, A. T., Kuryavyi, V., Gaw, H. Y. \& Patel, D. J. Small-molecule interaction with a five-guanine-tract G-quadruplex structure from the human MYC promoter. Nat. Chem. Biol. 1, 167-173 (2005).

129. Schnarr, L., Jana, J., Preckwinkel, P. \& Weisz, K. Impact of a Snap-Back Loop on Stability and Ligand Binding to a Parallel G-Quadruplex. J. Phys. Chem. B 124, 2778-2787 (2020).

130. Phan, A. T., Kuryavyi, V., Burge, S., Neidle, S. \& Patel, D. J. Structure of an Unprecedented G-Quadruplex Scaffold in the Human c-kit Promoter. J. Am. Chem. Soc. 129, 4386-4392 (2007).

131. Chen, Y. et al. The major G-quadruplex formed in the human platelet-derived growth factor receptor $\beta$ promoter adopts a novel broken-strand structure in K+ solution. J. Am. Chem. Soc. 134, 13220-3 (2012).

132. Chung, W. J. et al. Structure of a left-handed DNA G-quadruplex. Proc. Natl. Acad. Sci. 112, 2729-2733 (2015).

133. Fu, B. et al. Right-handed and left-handed G-quadruplexes have the same DNA sequence: distinct conformations induced by an organic small molecule and potassium. Chem. Commun. 52, 10052-10055 (2016).

134. Bakalar, B., Heddi, B., Schmitt, E., Mechulam, Y. \& Phan, A. T. A Minimal Sequence for Left-Handed G-Quadruplex Formation. Angew. Chem. Int. Ed. 58, 2331-2335 (2019).

135. Winnerdy, F. R. et al. NMR solution and X-ray crystal structures of a DNA molecule containing both right- and left-handed parallel-stranded G-quadruplexes. Nucleic Acids Res. 47, 8272-8281 (2019).

136. Escaja, N., Gómez-Pinto, I., Pedroso, E. \& González, C. Four-Stranded DNA Structures Can Be Stabilized by Two Different Types of Minor Groove G:C:G:C Tetrads. J. Am. Chem. Soc. 129, 2004-2014 (2007).

137. Kocman, V. \& Plavec, J. A tetrahelical DNA fold adopted by tandem repeats of alternating GGG and GCG tracts. Nat. Commun. 5, 5831 (2014).

138. Kocman, V. \& Plavec, J. Tetrahelical structural family adopted by AGCGA-rich regulatory DNA regions. Nat. Commun. 8, 15355 (2017).

139. Hadži, S., Kocman, V., Oblak, D., Plavec, J. \& Lah, J. Energetic Basis of AGCGA-Rich DNA Folding into a Tetrahelical Structure. Angew. Chem. Int. Ed. 58, 2387-2391 (2019).

140. Dejesus-Hernandez, M. et al. Expanded GGGGCC Hexanucleotide Repeat in Noncoding Region of C9ORF72 Causes Chromosome 9pLinked FTD and ALS. Neuron 72, 245-256 (2011).

141. Haeusler, A. R. et al. C9orf72 nucleotide repeat structures initiate molecular cascades of disease. Nature 507, 195-200 (2014).

142. Harkness, R. W. \& Mittermaier, A. K. G-quadruplex dynamics. Biochim. Biophys. Acta - Proteins Proteomics 1865, 1544-1554 (2017).

143. Phan, A. T. \& Patel, D. J. Two-Repeat Human Telomeric d(TAGGGTTAGGGT) Sequence Forms Interconverting Parallel and Antiparallel G-Quadruplexes in Solution: Distinct Topologies, Thermodynamic Properties, and Folding/Unfolding Kinetics. J. Am. Chem. Soc. 125, 15021-15027 (2003).

144. Bessi, I., Jonker, H. R. A., Richter, C. \& Schwalbe, H. Involvement of Long-Lived Intermediate States in the Complex Folding Pathway of the Human Telomeric G-Quadruplex. Angew. Chem. - Int. Ed. 54, 8444-8448 (2015).

145. Nußbaumer, F. et al. Synthesis and incorporation of 13C-labeled DNA building blocks to probe structural dynamics of DNA by NMR. Nucleic Acids Res. 45, 9178-9192 (2017).

146. Oh, K.-I., Kim, J., Park, C.-J. \& Lee, J.-H. Dynamics Studies of DNA with Non-canonical Structure Using NMR Spectroscopy. Int. J. Mol. Sci. 21, $2673(2020)$

147. Voter, A. F., Qiu, Y., Tippana, R., Myong, S. \& Keck, J. L. A guanine-flipping and sequestration mechanism for G-quadruplex unwinding by RecQ helicases. Nat. Commun. 9, 4201 (2018).

148. Cahoon, L. A. \& Seifert, H. S. An Alternative DNA Structure Is Necessary for Pilin Antigenic Variation in Neisseria gonorrhoeae. Science 325, 764-767 (2009)

149. Portella, G., Orozco, M. \& Vendruscolo, M. Determination of a Structural Ensemble Representing the Dynamics of a G-Quadruplex DNA. Biochemistry 59, 379-388 (2020).

150. Carrino, S., Hennecker, C. D., Murrieta, A. C. \& Mittermaier, A. Frustrated folding of guanine quadruplexes in telomeric DNA. Nucleic Acids Res. published online (2021). doi:10.1093/nar/gkab140

151. Levinthal, C. How to fold graciously. Mössbauer Spectrosc. Biol. Syst. Proc. 67, 22-26 (1969).

152. Levinthal, C. Are there pathways for protein folding? J. Chim. Phys. 65, 44-45 (1968).

153. Dill, K. A. \& Chan, H. S. From Levinthal to pathways to funnels. Nat. Struct. Mol. Biol. 4, 10-19 (1997)

154. Englander, S. W. \& Mayne, L. The nature of protein folding pathways. Proc. Natl. Acad. Sci. 111, 15873-15880 (2014).

155. Callaway, E. 'It will change everything': DeepMind's AI makes gigantic leap in solving protein structures. Nature 588, 203-204 (2020).

156. Anfinsen, C. B., Haber, E., Sela, M. \& White, F. H. The Kinetics Of Formation Of Native Ribonuclease During Oxidation Of The Reduced Polypeptide Chain. Proc. Natl. Acad. Sci. 47, 1309-1314 (1961).

157. Šponer, J. et al. Folding of guanine quadruplex molecules-funnel-like mechanism or kinetic partitioning? An overview from MD simulation studies. Biochim. Biophys. acta. Gen. Subj. 1861, 1246-1263 (2017).

158. Kuo, M. H.J. et al. Conformational Transition of a Hairpin Structure to G-Quadruplex within the WNT1 Gene Promoter. J. Am. Chem. Soc. 137, 210-218 (2015).

159. Frelih, T., Wang, B., Plavec, J. \& Šket, P. Pre-folded structures govern folding pathways of human telomeric G-quadruplexes. Nucleic Acids Res. 48, 2189-2197 (2020).

160. Kotar, A., Rigo, R., Sissi, C. \& Plavec, J. Two-quartet kit* G-quadruplex is formed via double-stranded pre-folded structure. Nucleic Acids Res. 47, 2641-2653 (2019).

161. Stadlbauer, P., Krepl, M., Cheatham, T. E., Koca, J. \& Sponer, J. Structural dynamics of possible late-stage intermediates in folding of quadruplex DNA studied by molecular simulations. Nucleic Acids Res. 41, 7128-7143 (2013). 


\section{References}

Stadlbauer, P., Trantírek, L., Cheatham, T. E., Koča, J. \& Šponer, J. Triplex intermediates in folding of human telomeric quadruplexes probed by microsecond-scale molecular dynamics simulations. Biochimie 105, 22-35 (2014).

Stadlbauer, P. et al. Parallel G-triplexes and G-hairpins as potential transitory ensembles in the folding of parallel-stranded DNA GQuadruplexes. Nucleic Acids Res. 47, 7276-7293 (2019).

Stadlbauer, P. et al. Hairpins participating in folding of human telomeric sequence quadruplexes studied by standard and T-REMD simulations. Nucleic Acids Res. 43, 9626-44 (2015).

Gajarský, M. et al. Structure of a Stable G-Hairpin. J. Am. Chem. Soc. 139, 3591-3594 (2017).

Živković, M. L. et al. Insight into formation propensity of pseudocircular DNA G-hairpins. Nucleic Acids Res. 49, 2317-2332 (2021).

Marchand, A. \& Erie Gabelica, V. Folding and misfolding pathways of G-quadruplex DNA. Nucleic Acids Res. 44, 10999-11012 (2016).

Gabelica, V. A pilgrim's guide to G-quadruplex nucleic acid folding. Biochimie 105, 1-3 (2014).

Gray, R. D., Trent, J. O. \& Chaires, J. B. Folding and Unfolding Pathways of the Human Telomeric G-Quadruplex. J. Mol. Biol. 426, 16291650 (2014).

Gray, R. D., Trent, J. O., Arumugam, S. \& Chaires, J. B. Folding Landscape of a Parallel G-Quadruplex. J. Phys. Chem. Lett. 10, 1146-1151 (2019).

Zhang, A. Y. Q. \& Balasubramanian, S. The kinetics and folding pathways of intramolecular G-quadruplex nucleic acids. J. Am. Chem. Soc. 134, 19297-19308 (2012).

Müller, D., Bessi, I., Richter, C. \& Schwalbe, H. The folding landscapes of human telomeric RNA and DNA G-quadruplexes are markedly different. Angew. Chem. Int. Ed. ange.202100280 (2021). doi:10.1002/ange.202100280

Aznauryan, M., Søndergaard, S., Noer, S. L., Schiøtt, B. \& Birkedal, V. A direct view of the complex multi-pathway folding of telomeric Gquadruplexes. Nucleic Acids Res. 44, 11024-11032 (2016).

Noer, S. L. et al. Folding dynamics and conformational heterogeneity of human telomeric G-quadruplex structures in $\mathrm{Na}+$ solutions by single molecule FRET microscopy. Nucleic Acids Res. 44, 464-71 (2016).

Li, W., Hou, X.-M., Wang, P.-Y., Xi, X.-G. \& Li, M. Direct Measurement of Sequential Folding Pathway and Energy Landscape of Human Telomeric G-quadruplex Structures. J. Am. Chem. Soc. 135, 6423-6426 (2013).

Cheng, Y. et al. Folding/unfolding kinetics of G-quadruplexes upstream of the P1 promoter of the human BCL-2 oncogene. J. Biol. Chem. 294, 5890-5895 (2019).

Gray, R. D. \& Chaires, J. B. Isothermal folding of G-quadruplexes. Methods 57, 47-55 (2012).

Mergny, J.-L. \& Lacroix, L. Analysis of Thermal Melting Curves. Oligonucleotides 13, 515-537 (2003).

Brown, N. M., Rachwal, P. A., Brown, T. \& Fox, K. R. Exceptionally slow kinetics of the intramolecular quadruplex formed by the Oxytricha telomeric repeat. Org. Biomol. Chem. 3, 4153-7 (2005).

Green, J. J., Ying, L., Klenerman, D. \& Balasubramanian, S. Kinetics of unfolding the human telomeric DNA quadruplex using a PNA trap. J. Am. Chem. Soc. 125, 3763-7 (2003).

Risitano, A. \& Fox, K. R. Stability of intramolecular DNA quadruplexes: comparison with DNA duplexes. Biochemistry 42, 6507-13 (2003). Lane, A. N. The stability of intramolecular DNA G-quadruplexes compared with other macromolecules. Biochimie 94, 277-86 (2012). Spoerner, M. et al. High pressure 31P NMR spectroscopy on guanine nucleotides. J. Biomol. NMR 67, 1-13 (2017).

Munte, C. E. et al. High pressure response of 1H NMR chemical shifts of purine nucleotides. Biophys. Chem. 254, 106261 (2019).

You, H., Wu, J., Shao, F. \& Yan, J. Stability and Kinetics of c- MYC Promoter G-Quadruplexes Studied by Single-Molecule Manipulation. J. Am. Chem. Soc. 137, 2424-2427 (2015).

Bugaut, A., Murat, P. \& Balasubramanian, S. An RNA Hairpin to G-Quadruplex Conformational Transition. J. Am. Chem. Soc. 134, 1995319956 (2012).

Wang, Z.-F. et al. Cytosine epigenetic modification modulates the formation of an unprecedented G4 structure in the WNT1 promoter. Nucleic Acids Res. 48, 1120-1130 (2020).

Lu, X.-M. et al. Folding Dynamics of Parallel and Antiparallel G-Triplexes under the Influence of Proximal DNA. J. Phys. Chem. B 122, 94999506 (2018).

Søndergaard, S. et al. Dynamics of Fluorescent Dyes Attached to G-Quadruplex DNA and their Effect on FRET Experiments. ChemPhysChem 16, 2562-2570 (2015).

Bhattacharyya, D., Mirihana Arachchilage, G. \& Basu, S. Metal Cations in G-Quadruplex Folding and Stability. Front. Chem. 4, (2016).

You, J. et al. Effects of monovalent cations on folding kinetics of G-quadruplexes. Biosci. Rep. 37, (2017).

Nguyen, T. Q. N., Lim, K. W. \& Phan, A. T. Folding Kinetics of G-Quadruplexes: Duplex Stem Loops Drive and Accelerate G-Quadruplex Folding. J. Phys. Chem. B 124, 5122-5130 (2020).

Hou, X.-M. et al. Involvement of G-triplex and G-hairpin in the multi-pathway folding of human telomeric G-quadruplex. Nucleic Acids Res. 45, 11401-11412 (2017).

Cerofolini, L. et al. G-triplex structure and formation propensity. Nucleic Acids Res. 42, 13393-13404 (2014).

Mashimo, T., Yagi, H., Sannohe, Y., Rajendran, A. \& Sugiyama, H. Folding Pathways of Human Telomeric Type-1 and Type-2 G-Quadruplex Structures. J. Am. Chem. Soc. 132, 14910-14918 (2010).

Lane, A. N., Chaires, J. B., Gray, R. D. \& Trent, J. O. Stability and kinetics of G-quadruplex structures. Nucleic Acids Res. 36, 5482-5515 (2008). Liu, W. et al. Kinetics and Mechanism of Conformational Changes in a G-Quadruplex of Thrombin-Binding Aptamer Induced by Pb2+. J. Phys. Chem. B 115, 13051-13056 (2011).

8. Liu, W. et al. Kinetics and mechanism of G-quadruplex formation and conformational switch in a G-quadruplex of PS2.M induced by Pb2+. Nucleic Acids Res. 40, 4229-4236 (2012).

Long, X. \& Stone, M. D. Kinetic Partitioning Modulates Human Telomere DNA G-Quadruplex Structural Polymorphism. PLoS One 8, e83420 (2013).

Gray, R. D. \& Chaires, J. B. Kinetics and mechanism of K+- and Na+-induced folding of models of human telomeric DNA into G-quadruplex structures. Nucleic Acids Res. 36, 4191-4203 (2008).

Xue, Y. et al. Kinetic and thermodynamic control of G-quadruplex folding. Angew. Chem. Int. Ed. 50, 8046-50 (2011).

Bian, Y. et al. Atomistic Picture for the Folding Pathway of a Hybrid-1 Type Human Telomeric DNA G-quadruplex. PLoS Comput. Biol. 10, e1003562 (2014).

Rocca, R. et al. Folding intermediate states of the parallel human telomeric G-quadruplex DNA explored using Well-Tempered Metadynamics. Sci. Rep. 10, 3176 (2020). 


\section{References}

204. Bian, Y. et al. Insights into the Kinetic Partitioning Folding Dynamics of the Human Telomeric G-Quadruplex from Molecular Simulations and Machine Learning. J. Chem. Theory Comput. 16, 5936-5947 (2020).

205. Wang, Y. \& Patel, D. J. Solution structure of the human telomeric repeat d[AG3(T2AG3)3] G-tetraplex. Structure 1, 263-282 (1993).

206. Parkinson, G. N., Lee, M. P. H. \& Neidle, S. Crystal structure of parallel quadruplexes from human telomeric DNA. Nature 417, 876-880 (2002).

207. Dai, J., Carver, M., Punchihewa, C., Jones, R. A. \& Yang, D. Structure of the hybrid-2 type intramolecular human telomeric G-quadruplex in K+ solution: Insights into structure polymorphism of the human telomeric sequence. Nucleic Acids Res. 35, 4927-4940 (2007).

208. Dai, J. et al. Structure of the intramolecular human telomeric G-quadruplex in potassium solution: a novel adenine triple formation. Nucleic Acids Res. 35, 2440-2450 (2007).

209. Phan, A. T., Kuryavyi, V., Luu, K. N. \& Patel, D. J. Structure of two intramolecular G-quadruplexes formed by natural human telomere sequences in K+ solution. Nucleic Acids Res. 35, 6517-25 (2007).

210. Luu, K. N., Phan, A. T., Kuryavyi, V., Lacroix, L. \& Patel, D. J. Structure of the Human Telomere in K+ Solution: An Intramolecular (3+1) GQuadruplex Scaffold. J. Am. Chem. Soc. 128, 9963-9970 (2006).

211. Isambert, H. The jerky and knotty dynamics of RNA. Methods 49, 189-196 (2009).

212. Kolesnikova, S., Hubálek, M., Bednárová, L., Cvačka, J. \& Curtis, E. A. Multimerization rules for G-quadruplexes. Nucleic Acids Res. 45, 86848696 (2017).

213. Mergny, J.-L. Kinetics of tetramolecular quadruplexes. Nucleic Acids Res. 33, 81-94 (2005).

214. Bardin, C. \& Leroy, J. L. The formation pathway of tetramolecular G-quadruplexes. Nucleic Acids Res. 36, 477-488 (2008).

215. Merkina, E. E. \& Fox, K. R. Kinetic Stability of Intermolecular DNA Quadruplexes. Biophys. J. 89, 365-373 (2005).

216. Hazel, P., Huppert, J., Balasubramanian, S. \& Neidle, S. Loop-Length-Dependent Folding of G-Quadruplexes. J. Am. Chem. Soc. 126, 1640516415 (2004)

217. Agrawal, P., Lin, C., Mathad, R. I., Carver, M. \& Yang, D. The major G-quadruplex formed in the human BCL-2 proximal promoter adopts a parallel structure with a 13-nt loop in K+ solution. J. Am. Chem. Soc. 136, 1750-3 (2014).

218. Greco, M. L. et al. Coexistence of two main folded G-quadruplexes within a single G-rich domain in the EGFR promoter. Nucleic Acids Res. 45, 10132-10142 (2017).

219. Balkwill, G. D., Garner, T. P. \& Searle, M. S. Folding of single-stranded DNA quadruplexes containing an autonomously stable mini-hairpin loop. Mol. Biosyst. 5, 542-7 (2009).

220. Zhang, D.-H. et al. Monomorphic RNA G-Quadruplex and Polymorphic DNA G-Quadruplex Structures Responding to Cellular Environmental Factors. Biochemistry 49, 4554-4563 (2010).

221. Tang, C.-F. \& Shafer, R. H. Engineering the Quadruplex Fold: Nucleoside Conformation Determines Both Folding Topology and Molecularity in Guanine Quadruplexes. J. Am. Chem. Soc. 128, 5966-5973 (2006).

222. Małgowska, M. Overview of RNA G-quadruplex structures. Acta Biochim. Pol. 63, (2017).

223. Mendoza, O., Porrini, M., Salgado, G. F., Gabelica, V. \& Mergny, J.-L. Orienting Tetramolecular G-Quadruplex Formation: The Quest for the Elusive RNA Antiparallel Quadruplex. Chem. Eur. J. 21, 6732-6739 (2015).

224. Xiao, C.-D., Ishizuka, T. \& Xu, Y. Antiparallel RNA G-quadruplex Formed by Human Telomere RNA Containing 8-Bromoguanosine. Sci. Rep. 7, 6695 (2017).

225. Karg, B., Haase, L., Funke, A., Dickerhoff, J. \& Weisz, K. Observation of a Dynamic G-Tetrad Flip in Intramolecular G-Quadruplexes. Biochemistry 55, 6949-6955 (2016).

226. Hirashima, K. \& Seimiya, H. Telomeric repeat-containing RNA/G-quadruplex-forming sequences cause genome-wide alteration of gene expression in human cancer cells in vivo. Nucleic Acids Res. 43, 2022-2032 (2015).

227. Martadinata, H. \& Phan, A. T. Structure of propeller-type parallel-stranded RNA G-quadruplexes, formed by human telomeric RNA sequences in K+ solution. J. Am. Chem. Soc. (2009). doi:10.1021/ja806592z

228. Phan, A. T. Human telomeric G-quadruplex: structures of DNA and RNA sequences. FEBS J. 277, 1107-17 (2010).

229. Collie, G. W. et al. Electrospray mass spectrometry of telomeric RNA (TERRA) reveals the formation of stable multimeric G-quadruplex structures. J. Am. Chem. Soc. 132, 9328-34 (2010).

230. Martadinata, H., Heddi, B., Lim, K. W. \& Phan, A. T. Structure of long human telomeric RNA (TERRA): G-quadruplexes formed by four and eight UUAGGG repeats are stable building blocks. Biochemistry 50, 6455-61 (2011).

231. Martadinata, H. \& Phan, A. T. Structure of Human Telomeric RNA (TERRA): Stacking of Two G-Quadruplex Blocks in K + Solution. Biochemistry 52, 2176-2183 (2013).

232. Dhakal, S. et al. G-Quadruplex and i-Motif Are Mutually Exclusive in ILPR Double-Stranded DNA. Biophys. J. 102, 2575-2584 (2012).

233. Cui, Y., Kong, D., Ghimire, C., Xu, C. \& Mao, H. Mutually Exclusive Formation of G-Quadruplex and i-Motif Is a General Phenomenon Governed by Steric Hindrance in Duplex DNA. Biochemistry 55, 2291-2299 (2016).

234. King, J. J. et al. DNA G-Quadruplex and i-Motif Structure Formation Is Interdependent in Human Cells. J. Am. Chem. Soc. 142, 20600-20604 (2020).

235. Sengupta, P., Bose, D. \& Chatterjee, S. The Molecular Tête-à-Tête between G-Quadruplexes and the i-motif in the Human Genome. ChemBioChem cbic.202000703 (2021). doi:10.1002/cbic.202000703

236. Kang, H.-J., Kendrick, S., Hecht, S. M. \& Hurley, L. H. The Transcriptional Complex Between the BCL2 i-Motif and hnRNP LL Is a Molecular Switch for Control of Gene Expression That Can Be Modulated by Small Molecules. J. Am. Chem. Soc. 136, 4172-4185 (2014).

237. Kaiser, C. E. et al. Insight into the Complexity of the i-Motif and G-Quadruplex DNA Structures Formed in the KRAS Promoter and Subsequent Drug-Induced Gene Repression. J. Am. Chem. Soc. 139, 8522-8536 (2017).

238. Kendrick, S. et al. The Dynamic Character of the BCL2 Promoter i-Motif Provides a Mechanism for Modulation of Gene Expression by Compounds That Bind Selectively to the Alternative DNA Hairpin Structure. J. Am. Chem. Soc. 136, 4161-4171 (2014).

239. Brooks, T. A., Kendrick, S. \& Hurley, L. Making sense of G-quadruplex and i-motif functions in oncogene promoters. FEBS J. 277, 3459-3469 (2010).

240. Sutherland, C., Cui, Y., Mao, H. \& Hurley, L. H. A Mechanosensor Mechanism Controls the G-Quadruplex/i-Motif Molecular Switch in the MYC Promoter NHE III 1. J. Am. Chem. Soc. 138, 14138-14151 (2016).

241. Dai, J., Hatzakis, E., Hurley, L. H. \& Yang, D. I-Motif Structures Formed in the Human c-MYC Promoter Are Highly Dynamic-Insights into Sequence Redundancy and I-Motif Stability. PLoS One 5, e11647 (2010).

242. Rhodes, D. \& Lipps, H. J. G-quadruplexes and their regulatory roles in biology. Nucleic Acids Res. 43, 8627-37 (2015).

243. Huppert, J. L. \& Balasubramanian, S. Prevalence of quadruplexes in the human genome. Nucleic Acids Res. 33, 2908-16 (2005).

244. Huppert, J. L. \& Balasubramanian, S. G-quadruplexes in promoters throughout the human genome. Nucleic Acids Res. 35, 406-413 (2007). 


\section{References}

Du, Z., Zhao, Y. \& Li, N. Genome-wide analysis reveals regulatory role of G4 DNA in gene transcription. Genome Res. 18, 233-241 (2008). Hänsel-Hertsch, R., Di Antonio, M. \& Balasubramanian, S. DNA G-quadruplexes in the human genome: Detection, functions and therapeutic potential. Nat. Rev. Mol. Cell Biol. 18, 279-284 (2017).

Maizels, N. \& Gray, L. T. The G4 Genome. PLoS Genet. 9, e1003468 (2013).

Todd, A. K., Johnston, M. \& Neidle, S. Highly prevalent putative quadruplex sequence motifs in human DNA. Nucleic Acids Res. 33, 29012907 (2005).

Eddy, J. \& Maizels, N. Gene function correlates with potential for G4 DNA formation in the human genome. Nucleic Acids Res. 34, 3887-3896 (2006).

Vallur, A. C. \& Maizels, N. Activities of human exonuclease 1 that promote cleavage of transcribed immunoglobulin switch regions. Proc. Natl. Acad. Sci. 105, 16508-16512 (2008).

Maizels, N. Dynamic roles for G4 DNA in the biology of eukaryotic cells. Nat. Struct. Mol. Biol. 13, 1055-1059 (2006).

Moyzis, R. K. et al. A highly conserved repetitive DNA sequence, (TTAGGG)n, present at the telomeres of human chromosomes. Proc. Natl. Acad. Sci. 85, 6622-6626 (1988).

Ribeyre, C. et al. The Yeast Pif1 Helicase Prevents Genomic Instability Caused by G-Quadruplex-Forming CEB1 Sequences In Vivo. PLoS Genet. 5, e1000475 (2009).

Fleming, A. M. et al. Human DNA Repair Genes Possess Potential G-Quadruplex Sequences in Their Promoters and 5'-Untranslated Regions. Biochemistry 57, 991-1002 (2018).

Mao, S.-Q. et al. DNA G-quadruplex structures mold the DNA methylome. Nat. Struct. Mol. Biol. 25, 951-957 (2018).

Guilbaud, G. et al. Local epigenetic reprogramming induced by G-quadruplex ligands. Nat. Chem. 9, 1110-1117 (2017).

Hänsel-Hertsch, R. et al. G-quadruplex structures mark human regulatory chromatin. Nat. Genet. 48, 1267-1272 (2016).

Reina, C. \& Cavalieri, V. Epigenetic Modulation of Chromatin States and Gene Expression by G-Quadruplex Structures. Int. J. Mol. Sci. 21, $4172(2020)$.

Varshney, D., Spiegel, J., Zyner, K., Tannahill, D. \& Balasubramanian, S. The regulation and functions of DNA and RNA G-quadruplexes. Nat. Rev. Mol. Cell Biol. 21, 459-474 (2020).

Bedrat, A., Lacroix, L. \& Mergny, J.-L. Re-evaluation of G-quadruplex propensity with G4Hunter. Nucleic Acids Res. 44, 1746-1759 (2016). Brázda, V. et al. G4Hunter web application: a web server for G-quadruplex prediction. Bioinformatics 35, 3493-3495 (2019).

Chambers, V. S. et al. High-throughput sequencing of DNA G-quadruplex structures in the human genome. Nat. Biotechnol. 33, 877-881 (2015).

Kikin, O., D’Antonio, L. \& Bagga, P. S. QGRS Mapper: a web-based server for predicting G-quadruplexes in nucleotide sequences. Nucleic Acids Res. 34, W676-W682 (2006).

Piazza, A. et al. Genetic instability triggered by G-quadruplex interacting Phen-DC compounds in Saccharomyces cerevisiae. Nucleic Acids Res. 38, 4337-4348 (2010).

Lopes, J. et al. G-quadruplex-induced instability during leading-strand replication. EMBO J. 30, 4033-4046 (2011).

Piazza, A. et al. Stimulation of Gross Chromosomal Rearrangements by the Human CEB1 and CEB25 Minisatellites in Saccharomyces cerevisiae Depends on G-Quadruplexes or Cdc13. PLoS Genet. 8, e1003033 (2012).

Piazza, A. et al. Short loop length and high thermal stability determine genomic instability induced by G-quadruplex-forming minisatellites. EMBO J. 34, 1718-1734 (2015).

Chai, W., Du, Q., Shay, J. W. \& Wright, W. E. Human telomeres have different overhang sizes at leading versus lagging strands. Mol. Cell 21, 427-435 (2006).

Lu, W., Zhang, Y., Liu, D., Songyang, Z. \& Wan, M. Telomeres-structure, function, and regulation. Experimental Cell Research 319, 133-141 (2013).

Morin, G. B. The human telomere terminal transferase enzyme is a ribonucleoprotein that synthesizes TTAGGG repeats. Cell 59, 521-529 (1989).

Sandin, S. \& Rhodes, D. Telomerase structure. Current Opinion in Structural Biology 25, 104-110 (2014).

Sfeir, A. J., Chai, W., Shay, J. W. \& Wright, W. E. Telomere-end processing the terminal nucleotides of human chromosomes. Mol. Cell 18, 131-8 (2005).

De Lange, T. Shelterin: The protein complex that shapes and safeguards human telomeres. Genes and Development 19, 2100-2110 (2005).

De Cian, A. et al. Targeting telomeres and telomerase. Biochimie 90, 131-55 (2008).

Hockemeyer, D. \& Collins, K. Control of telomerase action at human telomeres. Nat. Struct. Mol. Biol. 22, 848-52 (2015).

Wheelhouse, R. T., Sun, D., Han, H., Han, F. X. \& Hurley, L. H. Cationic Porphyrins as Telomerase Inhibitors: the Interaction of Tetra-( N methyl-4-pyridyl)porphine with Quadruplex DNA. J. Am. Chem. Soc. 120, 3261-3262 (1998).

Reed, J. E., Arnal, A. A., Neidle, S. \& Vilar, R. Stabilization of G-quadruplex DNA and inhibition of telomerase activity by square-planar nickel(II) complexes. J. Am. Chem. Soc. 128, 5992-5993 (2006).

Read, M. A. et al. Molecular modeling studies on G-quadruplex complexes of telomerase inhibitors: Structure-activity relationships. J. Med. Chem. 42, 4538-4546 (1999).

Ou, T. et al. G-quadruplexes: targets in anticancer drug design. ChemMedChem 3, 690-713 (2008).

Kim, M.-Y., Vankayalapati, H., Shin-Ya, K., Wierzba, K. \& Hurley, L. H. Telomestatin, a potent telomerase inhibitor that interacts quite specifically with the human telomeric intramolecular g-quadruplex. J. Am. Chem. Soc. 124, 2098-9 (2002).

Bazzicalupi, C., Ferraroni, M., Bilia, A. R., Scheggi, F. \& Gratteri, P. The crystal structure of human telomeric DN
An interesting case of stacked ligand to G-tetrad ratio higher than 1:1. Nucleic Acids Res. 41, 632-638 (2013).

Ohnmacht, S. A. \& Neidle, S. Small-molecule quadruplex-targeted drug discovery. Bioorganic Med. Chem. Lett. 24, 2602-2612 (2014).

McLuckie, K. I. E. et al. G-quadruplex DNA as a molecular target for induced synthetic lethality in cancer cells. J. Am. Chem. Soc. 135, 9640-3 (2013).

Sun, D. et al. Inhibition of Human Telomerase by a G-Quadruplex-Interactive Compound. J. Med. Chem. 40, 2113-2116 (1997).

Oganesian, L. \& Bryan, T. M. Physiological relevance of telomeric G-quadruplex formation: a potential drug target. BioEssays 29, 155-165 (2007).

Incles, C. M. et al. A G-quadruplex telomere targeting agent produces p16-associated senescence and chromosomal fusions in human prostate cancer cells. Mol. Cancer Ther. 3, 1201-6 (2004).

Tauchi, T. et al. Activity of a novel G-quadruplex-interactive telomerase inhibitor, telomestatin (SOT-095), against human leukemia cells: involvement of ATM-dependent DNA damage response pathways. Oncogene 22, 5338-5347 (2003). 


\section{References}

288. Salvati, E. et al. Telomere damage induced by the G-quadruplex ligand RHPS4 has an antitumor effect. J. Clin. Invest. 117, 3236-3247 (2007).

Harrison, R. J., Gowan, S. M., Kelland, L. R. \& Neidle, S. Human telomerase inhibition by substituted acridine derivatives. Bioorg. Med. Chem. Lett. 9, 2463-2468 (1999).

290. Bryan, T. M. G-Quadruplexes at Telomeres: Friend or Foe? Molecules 25, 3686 (2020).

291. Carvalho, J., Mergny, J.-L., Salgado, G. F., Queiroz, J. A. \& Cruz, C. G-quadruplex, Friend or Foe: The Role of the G-quartet in Anticancer Strategies. Trends Mol. Med. 26, 848-861 (2020).

292. Nakanishi, C. \& Seimiya, H. G-quadruplex in cancer biology and drug discovery. Biochem. Biophys. Res. Commun. 531, 45-50 (2020).

293. Zhao, J. \& Zhai, Q. Recent advances in the development of ligands specifically targeting telomeric multimeric G-quadruplexes. Bioorg. Chem. 103, 104229 (2020).

294. Hänsel-Hertsch, R., Spiegel, J., Marsico, G., Tannahill, D. \& Balasubramanian, S. Genome-wide mapping of endogenous G-quadruplex DNA structures by chromatin immunoprecipitation and high-throughput sequencing. Nat. Protoc. 13, 551-564 (2018).

295. Hänsel-Hertsch, R. et al. Landscape of G-quadruplex DNA structural regions in breast cancer. Nat. Genet. 52, 878-883 (2020).

296. Schaffitzel, C. et al. In vitro generated antibodies specific for telomeric guanine-quadruplex DNA react with Stylonychia lemnae macronuclei. Proc. Natl. Acad. Sci. 98, 8572-8577 (2001).

297. Paeschke, K. et al. Telomerase recruitment by the telomere end binding protein- $\beta$ facilitates G-quadruplex DNA unfolding in ciliates. Nat. Struct. Mol. Biol. 15, 598-604 (2008).

298. Henderson, A. et al. Detection of G-quadruplex DNA in mammalian cells. Nucleic Acids Res. 42, 860-869 (2014).

299. Liu, H.-Y. et al. Conformation Selective Antibody Enables Genome Profiling and Leads to Discovery of Parallel G-Quadruplex in Human Telomeres. Cell Chem. Biol. 23, 1261-1270 (2016).

300. Hänsel, R. et al. Evaluation of Parameters Critical for Observing Nucleic Acids Inside Living Xenopus laevis Oocytes by In-Cell NMR Spectroscopy. J. Am. Chem. Soc. 131, 15761-15768 (2009).

301. Hänsel, R. et al. The parallel G-quadruplex structure of vertebrate telomeric repeat sequences is not the preferred folding topology under physiological conditions. Nucleic Acids Res. 39, 5768-5775 (2011).

302. Hänsel, R., Foldynová-Trantírková, S., Dötsch, V. \& Trantírek, L. Investigation of quadruplex structure under physiological conditions using in-cell NMR. Topics in Current Chemistry 330, 47-66 (2013).

303. Hänsel, R., Löhr, F., Trantirek, L. \& Dötsch, V. High-resolution insight into G-overhang architecture. J. Am. Chem. Soc. 135, 2816-24 (2013).

304. Hänsel, R., Luh, L. M., Corbeski, I., Trantirek, L. \& Dötsch, V. In-cell NMR and EPR spectroscopy of biomacromolecules. Angew. Chem. Int. Ed. 53, 10300-14 (2014).

305. Salgado, G. F., Cazenave, C., Kerkour, A. \& Mergny, J.-L. G-quadruplex DNA and ligand interaction in living cells using NMR spectroscopy. Chem. Sci. 6, 3314-3320 (2015).

306. Yamaoki, Y., Nagata, T., Sakamoto, T. \& Katahira, M. Recent progress of in-cell NMR of nucleic acids in living human cells. Biophys. Rev. 12, $411-417$ (2020).

307. Bao, H.-L. et al. Characterization of human telomere RNA G-quadruplex structures in vitro and in living cells using 19F NMR spectroscopy. Nucleic Acids Res. 45, 5501-5511 (2017).

308. Bao, H.-L. \& Xu, Y. Investigation of higher-order RNA G-quadruplex structures in vitro and in living cells by 19F NMR spectroscopy. Nat. Protoc. 13, 652-665 (2018)

309. Bao, H.-L., Liu, H. \& Xu, Y. Hybrid-type and two-tetrad antiparallel telomere DNA G-quadruplex structures in living human cells. Nucleic Acids Res. 47, 4940-4947 (2019).

310. Adrian, M., Heddi, B. \& Phan, A. T. NMR spectroscopy of G-quadruplexes. Methods 57, 11-24 (2012).

311. Chilka, P., Desai, N. \& Datta, B. Small Molecule Fluorescent Probes for G- Quadruplex Visualization as Potential Cancer Theranostic Agents. Molecules 24, 752 (2019).

312. Huang, W.-C. et al. Direct evidence of mitochondrial G-quadruplex DNA by using fluorescent anti-cancer agents. Nucleic Acids Res. 43, 1010213 (2015).

313. Laguerre, A., Wong, J. M. Y. \& Monchaud, D. Direct visualization of both DNA and RNA quadruplexes in human cells via an uncommon spectroscopic method. Sci. Rep. 6, 32141 (2016).

314. Summers, P. A. et al. Visualising G-quadruplex DNA dynamics in live cells by fluorescence lifetime imaging microscopy. Nat. Commun. 12, 162 (2021).

315. Spiegel, J., Adhikari, S. \& Balasubramanian, S. The Structure and Function of DNA G-Quadruplexes. Trends in Chemistry 2, 123-136 (2020).

316. Bryan, T. M. Mechanisms of DNA Replication and Repair: Insights from the Study of G-Quadruplexes. Molecules 24, 3439 (2019).

317. Lerner, L. K. \& Sale, J. E. Replication of G Quadruplex DNA. Genes (Basel). 10, 95 (2019).

318. Prorok, P. et al. Involvement of G-quadruplex regions in mammalian replication origin activity. Nat. Commun. 10, 3274 (2019).

319. Valton, A.-L. \& Prioleau, M.-N. G-Quadruplexes in DNA Replication: A Problem or a Necessity? Trends Genet. 32, 697-706 (2016).

320. Tsukakoshi, K., Saito, S., Yoshida, W., Goto, S. \& Ikebukuro, K. CpG Methylation Changes G-Quadruplex Structures Derived from Gene Promoters and Interaction with VEGF and SP1. Molecules 23, 944 (2018).

321. Tu, A. T. T., Hoshi, K., Ikebukuro, K., Hanagata, N. \& Yamazaki, T. Monomeric G-Quadruplex-Based CpG Oligodeoxynucleotides as Potent Toll-Like Receptor 9 Agonists. Biomacromolecules 21, 3644-3657 (2020).

322. Jara-Espejo, M. \& Line, S. R. DNA G-quadruplex stability, position and chromatin accessibility are associated with CpG island methylation. FEBS J. 287, 483-495 (2020).

323. Yoshida, W., Terasaka, M., Laddachote, S. \& Karube, I. Stabilization of G-quadruplex structure on vascular endothelial growth factor gene promoter depends on CpG methylation site and cation type. Biochim. Biophys. Acta - Gen. Subj. 1862, 1933-1937 (2018).

324. Kim, N. The Interplay between G-quadruplex and Transcription. Curr. Med. Chem. 26, 2898-2917 (2019).

325. Tong, X. et al. Solution structure of all parallel G-quadruplex formed by the oncogene RET promoter sequence. Nucleic Acids Res. 39, 6753$6763(2011)$.

326. Agarwal, T., Roy, S., Kumar, S., Chakraborty, T. K. \& Maiti, S. In the sense of transcription regulation by G-quadruplexes: Asymmetric effects in sense and antisense strands. Biochemistry 53, 3711-3718 (2014).

327. Bourbon, H. M., Lapeyre, B. \& Amalric, F. Structure of the mouse nucleolin gene. The complete sequence reveals that each RNA binding domain is encoded by two independent exons. J. Mol. Biol. 200, 627-638 (1988).

328. Caizergues-Ferrer, M. et al. Nucleolin from Xenopus laevis: cDNA cloning and expression during development. Genes Dev. 3, 324-333 (1989).

329. Maridor, G., Krek, W. \& Nigg, E. A. Structure and developmental expression of chicken nucleolin and NO38: Coordinate expression of two abundant non-ribosomal nucleolar proteins. BBA - Gene Struct. Expr. 1049, 126-133 (1990). 


\section{References}

Peter, M., Nakagawa, J., Dorée, M., Labbé, J. C. \& Nigg, E. A. Identification of major nucleolar proteins as candidate mitotic substrates of cdc2 kinase. Cell 60, 791-801 (1990).

331.

332.

333.

334

335

336

Tuteja, R. \& Tuteja, N. Nucleolin: A Multifunctional Major Nucleolar Phosphoprotein. Crit. Rev. Biochem. Mol. Biol. 33, 407-436 (1998). Ginisty, H., Sicard, H., Roger, B. \& Bouvet, P. Structure and functions of nucleolin. J. Cell Sci. 112 ( Pt 6, 761-72 (1999). Tajrishi, M. M., Tuteja, R. \& Tuteja, N. Nucleolin. Commun. Integr. Biol. 4, 267-275 (2011). Hanakahi, L. A., Sun, H. \& Maizels, N. High Affinity Interactions of Nucleolin with G-G-paired rDNA. J. Biol. Chem. 274, 15908-15912 (1999).

González, V., Guo, K., Hurley, L. \& Sun, D. Identification and characterization of nucleolin as a c-myc G-quadruplex-binding protein. J. Biol. Chem. 284, 23622-35 (2009).

Tosoni, E. et al. Nucleolin stabilizes G-quadruplex structures folded by the LTR promoter and silences HIV-1 viral transcription. Nucleic Acids Res. 43, 8884-8897 (2015).

De Nicola, B. et al. Structure and possible function of a G-quadruplex in the long terminal repeat of the proviral HIV-1 genome. Nucleic Acids Res. 44, 6442-6451 (2016).

González, V. \& Hurley, L. H. The C-terminus of nucleolin promotes the formation of the c-MYC G-quadruplex and inhibits c-MYC promoter activity. Biochemistry 49, 9706-14 (2010).

Lago, S., Tosoni, E., Nadai, M., Palumbo, M. \& Richter, S. N. The cellular protein nucleolin preferentially binds long-looped G-quadruplex nucleic acids. Biochim. Biophys. Acta - Gen. Subj. 1861, 1371-1381 (2017).

Saha, A. et al. Nucleolin Discriminates Drastically between Long-Loop and Short-Loop Quadruplexes. Biochemistry 59, 1261-1272 (2020).

Sengupta, P., Bhattacharya, A., Sa, G., Das, T. \& Chatterjee, S. Truncated G-Quadruplex Isomers Cross-Talk with the Transcription Factors To Maintain Homeostatic Equilibria in c-MYC Transcription. Biochemistry 58, 1975-1991 (2019).

Dickerhoff, J., Onel, B., Chen, L., Chen, Y. \& Yang, D. Solution Structure of a MYC Promoter G-Quadruplex with 1:6:1 Loop Length. ACS Omega 4, 2533-2539 (2019).

Bian, W.-X. et al. Binding of cellular nucleolin with the viral core RNA G-quadruplex structure suppresses HCV replication. Nucleic Acids Res. 47, 56-68 (2019).

Santos, T. et al. Recognition of nucleolin through interaction with RNA G-quadruplex. Biochem. Pharmacol. 114208 (2020). doi:10.1016/j.bcp.2020.114208

Figueiredo, J. et al. Targeting nucleolin by RNA G-quadruplex-forming motif. Biochem. Pharmacol. 114418 (2021). doi:10.1016/j.bcp.2021.114418

Mendoza, O., Bourdoncle, A., Boulé, J. B., Brosh, R. M. \& Mergny, J. L. G-quadruplexes and helicases. Nucleic Acids Research 44, 1989-2006 (2016).

Wu, G., Xing, Z., Tran, E. J. \& Yang, D. DDX5 helicase resolves G-quadruplex and is involved in MYC gene transcriptional activation. Proc. Natl. Acad. Sci. 116, 20453-20461 (2019).

De Magis, A. et al. Zuo1 supports G4 structure formation and directs repair toward nucleotide excision repair. Nat. Commun. 11, 3907 (2020). Hároníková, L. et al. IFI16 Preferentially Binds to DNA with Quadruplex Structure and Enhances DNA Quadruplex Formation. PLoS One 11, e0157156 (2016).

Carle, C. M., Zaher, H. S. \& Chalker, D. L. A Parallel G Quadruplex-Binding Protein Regulates the Boundaries of DNA Elimination Events of Tetrahymena thermophila. PLOS Genet. 12, e1005842 (2016).

Niu, K. et al. Identification of LARK as a novel and conserved G-quadruplex binding protein in invertebrates and vertebrates. Nucleic Acids Res. 47, 7306-7320 (2019).

Williams, P., Li, L., Dong, X. \& Wang, Y. Identification of SLIRP as a G Quadruplex-Binding Protein. J. Am. Chem. Soc. 139, 12426-12429 (2017).

Chen, S. et al. Mechanistic studies for the role of cellular nucleic-acid-binding protein (CNBP) in regulation of c-myc transcription. Biochim. Biophys. Acta - Gen. Subj. 1830, 4769-4777 (2013).

David, A. P. et al. CNBP controls transcription by unfolding DNA G-quadruplex structures. Nucleic Acids Res. 47, 7901-7913 (2019).

Fry, M. Tetraplex DNA and its interacting proteins. Front. Biosci. 12, 4336-51 (2007).

Brázda, V., Hároníková, L., Liao, J. \& Fojta, M. DNA and RNA Quadruplex-Binding Proteins. Int. J. Mol. Sci. 15, 17493-17517 (2014).

Brázda, V. et al. The Amino Acid Composition of Quadruplex Binding Proteins Reveals a Shared Motif and Predicts New Potential Quadruplex Interactors. Molecules 23, 2341 (2018).

Huang, Z.-L. et al. Identification of G-Quadruplex-Binding Protein from the Exploration of RGG Motif/G-Quadruplex Interactions. J. Am. Chem. Soc. 140, 17945-17955 (2018).

Armas, P., Agüero, T. H., Borgognone, M., Aybar, M. J. \& Calcaterra, N. B. Dissecting CNBP, a Zinc-Finger Protein Required for Neural Crest Development, in Its Structural and Functional Domains. J. Mol. Biol. 382, 1043-1056 (2008).

Masuzawa, T. \& Oyoshi, T. Roles of the RGG Domain and RNA Recognition Motif of Nucleolin in G-Quadruplex Stabilization. ACS Omega 5, 5202-5208 (2020).

Mishra, S. K., Tawani, A., Mishra, A. \& Kumar, A. G4IPDB: A database for G-quadruplex structure forming nucleic acid interacting proteins. Sci. Rep. 6, 38144 (2016).

Heddi, B., Cheong, V. V., Martadinata, H. \& Phan, A. T. Insights into G-quadruplex specific recognition by the DEAH-box helicase RHAU: Solution structure of a peptide-quadruplex complex. Proc. Natl. Acad. Sci. 112, 9608-9613 (2015).

Chen, M. C. et al. Structural basis of G-quadruplex unfolding by the DEAH/RHA helicase DHX36. Nature 558, 465-469 (2018).

Cogoi, S., Shchekotikhin, A. E. \& Xodo, L. E. HRAS is silenced by two neighboring G-quadruplexes and activated by MAZ, a zinc-finger transcription factor with DNA unfolding property. Nucleic Acids Res. 42, 8379-8388 (2014).

Cogoi, S., Ferino, A., Miglietta, G., Pedersen, E. B. \& Xodo, L. E. The regulatory G4 motif of the Kirsten ras (KRAS) gene is sensitive to guanine oxidation: implications on transcription. Nucleic Acids Res. 46, 661-676 (2018).

Postel, E. H. Multiple biochemical activities of NM23/NDP kinase in gene regulation. J. Bioenerg. Biomembr. 35, 31-40 (2003).

Dexheimer, T. S. et al. NM23-H2 may play an indirect role in transcriptional activation of c-myc gene expression but does not cleave the nuclease hypersensitive element III 1. Mol. Cancer Ther. 8, 1363-1377 (2009).

Ma, D. et al. NM23-H1 and NM23-H2 Repress Transcriptional Activities of Nuclease-hypersensitive Elements in the Platelet-derived Growth Factor-A Promoter. J. Biol. Chem. 277, 1560-1567 (2002).

González, V. \& Hurley, L. H. The c- MYC NHE III 1 : Function and Regulation. Annu. Rev. Pharmacol. Toxicol. 50, 111-129 (2010). 


\section{References}

Sharma, S., Sengupta, A. \& Chowdhury, S. NM23/NDPK proteins in transcription regulatory functions and chromatin modulation: emerging trends. Lab. Investig. 98, 175-181 (2018).

371 Epigenetic suppression of human telomerase

dependent fashion. J. Biol. Chem. 292, 15205-15215 (2017).

Genomic Stability. Mol. Cell. Biol. 28, 4116-4128 (2008).

373. Sarkies, P. et al. FANCJ coordinates two pathways that maintain epigenetic stability at G-quadruplex DNA. Nucleic Acids Res. 40, 1485-1498 (2012).

374. Wu, C. G. \& Spies, M. G-quadruplex recognition and remodeling by the FANCJ helicase. Nucleic Acids Res. 44, 8742-8753 (2016).

375. Paeschke, K. et al. Pif1 family helicases suppress genome instability at G-quadruplex motifs. Nature 497, 458-462 (2013).

376. Byrd, A. K. \& Raney, K. D. Structure and function of Pif1 helicase. Biochem. Soc. Trans. 45, 1159-1171 (2017).

377. Li, J.-L.et al. Inhibition of the Bloom's and Werner's Syndrome Helicases by G-Quadruplex Interacting Ligands. Biochemistry 40, 15194-15202 (2001).

378. Wu, W. et al. HERC2 Facilitates BLM and WRN Helicase Complex Interaction with RPA to Suppress G-Quadruplex DNA. Cancer Res. 78, 6371-6385 (2018).

379. Smestad, J. A. \& Maher, L. J. Relationships between putative G-quadruplex-forming sequences, RecQ helicases, and transcription. BMC Med. Genet. 16, 91 (2015).

380. Wu, W.-Q., Hou, X.-M., Li, M., Dou, S.-X. \& Xi, X.-G. BLM unfolds G-quadruplexes in different structural environments through different mechanisms. Nucleic Acids Res. 43, 4614-4626 (2015).

381. Budhathoki, J. B., Stafford, E. J., Yodh, J. G. \& Balci, H. ATP-dependent G-quadruplex unfolding by Bloom helicase exhibits low processivity. Nucleic Acids Res. 43, 5961-5970 (2015).

382. Chatterjee, S. et al. Mechanistic insight into the interaction of BLM helicase with intra-strand G-quadruplex structures. Nat. Commun. 5, 5556 (2014).

383. van Wietmarschen, N. et al. BLM helicase suppresses recombination at G-quadruplex motifs in transcribed genes. Nat. Commun. 9, 271 (2018).

384. Lee, S., Kim, J., Han, S. \& Park, C.-J. Recognition and Unfolding of c-MYC and Telomeric G-Quadruplex DNAs by the RecQ C-Terminal Domain of Human Bloom Syndrome Helicase. ACS Omega 5, 14513-14522 (2020).

385. Aggarwal, M., Sommers, J. a, Shoemaker, R. H. \& Brosh, R. M. Inhibition of helicase activity by a small molecule impairs Werner syndrome helicase (WRN) function in the cellular response to DNA damage or replication stress. Proc. Natl. Acad. Sci. 108, 1525-1530 (2011).

386. Johnson, J. E., Cao, K., Ryvkin, P., Wang, L.-S. \& Johnson, F. B. Altered gene expression in the Werner and Bloom syndromes is associated with sequences having G-quadruplex forming potential. Nucleic Acids Res. 38, 1114-1122 (2010).

387. Budhathoki, J. B. et al. A Comparative Study of G-Quadruplex Unfolding and DNA Reeling Activities of Human RECQ5 Helicase. Biophys. J. 110, 2585-2596 (2016).

388. Keller, H. et al. The intrinsically disordered amino-terminal region of human RecQL4: multiple DNA-binding domains confer annealing, strand exchange and G4 DNA binding. Nucleic Acids Res. 42, 12614-12627 (2014).

389. Ghosh, A. K. et al. RECQL4, the protein mutated in Rothmund-Thomson syndrome, functions in telomere maintenance. J. Biol. Chem. 287, 196-209 (2012)

390. Castillo-Tandazo, W. et al. ATP-dependent helicase activity is dispensable for the physiological functions of Recq14. PLOS Genet. 15, e1008266 (2019).

391. Sauer, M. \& Paeschke, K. G-quadruplex unwinding helicases and their function in vivo. Biochem. Soc. Trans. 45, 1173-1182 (2017).

392. Tippana, R., Hwang, H., Opresko, P. L., Bohr, V. A. \& Myong, S. Single-molecule imaging reveals a common mechanism shared by Gquadruplex-resolving helicases. Proc. Natl. Acad. Sci. U. S. A. 113, 8448-8453 (2016).

393. Popuri, V. et al. The Human RecQ helicases, BLM and RECQ1, display distinct DNA substrate specificities. J. Biol. Chem. 283, 17766-76 (2008).

394. Ellis, N. A. et al. The Bloom's syndrome gene product is homologous to RecQhelicases. Cell 83, 655-666 (1995).

395. Gray, M. D. et al. The Werner syndrome protein is a DNA helicase. Nat. Genet. 17, 100-103 (1997).

396. Larizza, L., Magnani, I. \& Roversi, G. Rothmund-Thomson syndrome and RECQL4 defect: Splitting and lumping. Cancer Lett. 232, 107-120 (2006).

397. Majello, B., De Luca, P., Suske, G. \& Lania, L. Differential transcriptional regulation of c-myc promoter through the same DNA binding sites targeted by Sp1-like proteins. Oncogene 10, 1841-8 (1995).

398. Suske, G. The Sp-family of transcription factors. Gene 238, 291-300 (1999)

399. Han, H., Langley, D. R., Rangan, A. \& Hurley, L. H. Selective interactions of cationic porphyrins with G-quadruplex structures. J. Am. Chem. Soc. 123, 8902-13 (2001).

400. Haiyong, H., Cliff, C. L. \& Hurley, L. H. Accelerated assembly of G-quadruplex structures by a small molecule. Biochemistry 38, 6981-6986 (1999).

401. Izbicka, E. et al. Effects of cationic porphyrins as G-quadruplex interactive agents in human tumor cells. Cancer Res. 59, 639-44 (1999).

402. Tian, T., Chen, Y.-Q., Wang, S.-R. \& Zhou, X. G-Quadruplex: A Regulator of Gene Expression and Its Chemical Targeting. Chem 4, 13141344 (2018).

403. Bessi, I., Wirmer-Bartoschek, J., Dash, J. \& Schwalbe, H. Targeting G-quadruplex with Small Molecules: An NMR View. in Modern Magnetic Resonance 2189-2210 (Springer International Publishing, 2018). doi:10.1007/978-3-319-28388-3_126

404. Franceschin, M. et al. Natural and synthetic G-quadruplex interactive berberine derivatives. Bioorganic Med. Chem. Lett. 16, 1707-1711 (2006).

405. Shirude, P. S. et al. Macrocyclic and Helical Oligoamides as a New Class of G-Quadruplex Ligands. J. Am. Chem. Soc. 129, 11890-11891 (2007).

406. Monchaud, D. \& Teulade-Fichou, M.-P. A hitchhiker's guide to G-quadruplex ligands. Org. Biomol. Chem. 6, 627-36 (2008).

407. Burger, A. M. et al. The G-quadruplex-interactive molecule BRACO-19 inhibits tumor growth, consistent with telomere targeting and interference with telomerase function. Cancer Res. 65, 1489-1496 (2005).

408. Sun, D., Guo, K., Rusche, J. J. \& Hurley, L. H. Facilitation of a structural transition in the polypurine/polypyrimidine tract within the proximal promoter region of the human VEGF gene by the presence of potassium and G-quadruplex-interactive agents. Nucleic Acids Res. 33, 60706080 (2005).

409. Shin-ya, K. et al. Telomestatin, a Novel Telomerase Inhibitor from Streptomyces anulatus. J. Am. Chem. Soc. 123, 1262-1263 (2001).

410. Bessi, I. et al. Spectroscopic, molecular modeling, and NMR-spectroscopic investigation of the binding mode of the natural alkaloids berberine and sanguinarine to human telomeric G-quadruplex DNA. ACS Chem. Biol. 7, 1109-1119 (2012). 


\section{References}

411. Ruggiero, E. \& Richter, S. N. G-quadruplexes and G-quadruplex ligands: targets and tools in antiviral therapy. Nucleic Acids Res. 46, 3270-3283 (2018).

412. Duarte, A. R., Cadoni, E., Ressurreição, A. S., Moreira, R. \& Paulo, A. Design of Modular G-quadruplex Ligands. ChemMedChem 13, 869-893 (2018).

413. O'Hagan, M. P., Morales, J. C. \& Galan, M. C. Binding and Beyond: What Else Can G-Quadruplex Ligands Do? European J. Org. Chem. 2019, 4995-5017 (2019).

414. De Magis, A. et al. DNA damage and genome instability by G-quadruplex ligands are mediated by R loops in human cancer cells. Proc. Natl. Acad. Sci. 116, 816-825 (2019).

415. Li, Q. et al. G4LDB: a database for discovering and studying G-quadruplex ligands. Nucleic Acids Res. 41, D1115-D1123 (2013).

416. Wirmer-Bartoschek, J. et al. Solution NMR Structure of a Ligand/Hybrid-2-G-Quadruplex Complex Reveals Rearrangements that Affect Ligand Binding. Angew. Chem. Int. Ed. 56, 7102-7106 (2017).

417. Lejault, P., Mitteaux, J., Sperti, F. R. \& Monchaud, D. How to untie G-quadruplex knots and why? Cell Chem. Biol. in press (2021). doi:10.1016/j.chembiol.2021.01.015

418. Grand, C. L. et al. The cationic porphyrin TMPyP4 down-regulates c-MYC and human telomerase reverse transcriptase expression and inhibits tumor growth in vivo. Mol. Cancer Ther. 1, 565-73 (2002).

419. Kim, M.-Y., Gleason-Guzman, M., Izbicka, E., Nishioka, D. \& Hurley, L. H. The different biological effects of telomestatin and TMPyP4 can be attributed to their selectivity for interaction with intramolecular or intermolecular G-quadruplex structures. Cancer Res. 63, 3247-56 (2003). 420. Parkinson, G. N., Ghosh, R. \& Neidle, S. Structural basis for binding of porphyrin to human telomeres. Biochemistry 46, 2390-7 (2007).

421. Read, M. et al. Structure-based design of selective and potent G quadruplex-mediated telomerase inhibitors. Proc. Natl. Acad. Sci. U. S. A. 98, 4844-9 (2001).

422. Campbell, N. H., Parkinson, G. N., Reszka, A. P. \& Neidle, S. Structural Basis of DNA Quadruplex Recognition by an Acridine Drug. J. Am. Chem. Soc. 130, 6722-6724 (2008).

423. Monchaud, D. et al. Ligands playing musical chairs with G-quadruplex DNA: A rapid and simple displacement assay for identifying selective G-quadruplex binders. Biochimie 90, 1207-1223 (2008).

424. De Cian, A., DeLemos, E., Mergny, J.-L., Teulade-Fichou, M.-P. \& Monchaud, D. Highly Efficient G-Quadruplex Recognition by Bisquinolinium Compounds. J. Am. Chem. Soc. 129, 1856-1857 (2007).

425. Chung, W. J., Heddi, B., Hamon, F., Teulade-Fichou, M.-P. \& Phan, A. T. Solution structure of a G-quadruplex bound to the bisquinolinium compound Phen-DC(3). Angew. Chem. Int. Ed. 53, 999-1002 (2014).

426. Chung, W.J. et al. Solution Structure of an Intramolecular (3+1) Human Telomeric G-Quadruplex Bound to a Telomestatin Derivative. J. Am. Chem. Soc. 135, 13495-13501 (2013).

427. Casini, A. et al. Structural and solution chemistry, antiproliferative effects, and DNA and protein binding properties of a series of dinuclear gold(III) compounds with bipyridyl ligands. J. Med. Chem. 49, 5524-5531 (2006).

428. Gabbiani, C., Guerri, A., Cinellu, M. A. \& Messori, L. Dinuclear Gold(III) Complexes as Potential Anticancer Agents: Structure, Reactivity and Biological Profile of a Series of Gold(III) Oxo-Bridged Derivatives. Open Crystallogr. J. 3, 29-40 (2010).

429. Gratteri, P. et al. Interactions of selected gold(III) complexes with DNA G quadruplexes. Dalt. Trans. 44, 3633-9 (2015).

430. Heller, A. Spiers Memorial Lecture: On the hypothesis of cathodic protection of genes. Faraday Discuss. 116, 1-13 (2000).

431. Fleming, A. M. \& Burrows, C. J. 8-Oxo-7,8-dihydroguanine, friend and foe: Epigenetic-like regulator versus initiator of mutagenesis. DNA Repair (Amst). 56, 75-83 (2017).

432. Redstone, S. C. J., Fleming, A. M. \& Burrows, C. J. Oxidative Modification of the Potential G-Quadruplex Sequence in the PCNA Gene Promoter Can Turn on Transcription. Chem. Res. Toxicol. 32, 437-446 (2019).

433. Fleming, A. M. \& Burrows, C. J. G-Quadruplex Folds of the Human Telomere Sequence Alter the Site Reactivity and Reaction Pathway of Guanine Oxidation Compared to Duplex DNA. Chem. Res. Toxicol. 26, 593-607 (2013).

434. An, N., Fleming, A. M. \& Burrows, C. J. Human Telomere G-Quadruplexes with Five Repeats Accommodate 8-Oxo-7,8-dihydroguanine by Looping out the DNA Damage. ACS Chem. Biol. 11, 500-507 (2016).

435. Fleming, A. M., Ding, Y. \& Burrows, C. J. Oxidative DNA damage is epigenetic by regulating gene transcription via base excision repair. Proc. Natl. Acad. Sci. U. S. A. 114, 2604-2609 (2017).

436. Fleming, A. M., Zhu, J., Ding, Y. \& Burrows, C. J. 8-Oxo-7,8-dihydroguanine in the Context of a Gene Promoter G-Quadruplex Is an On-Off Switch for Transcription. ACS Chem. Biol. 12, 2417-2426 (2017).

437. Zhu, J., Fleming, A. M. \& Burrows, C. J. The RAD17 Promoter Sequence Contains a Potential Tail-Dependent G-Quadruplex That Downregulates Gene Expression upon Oxidative Modification. ACS Chem. Biol. 13, 2577-2584 (2018).

438. Omaga, C. A., Fleming, A. M. \& Burrows, C. J. The Fifth Domain in the G-Quadruplex-Forming Sequence of the Human NEIL3 Promoter Locks DNA Folding in Response to Oxidative Damage. Biochemistry 57, 2958-2970 (2018).

439. Fleming, A. M., Zhu, J., Howpay Manage, S. A. \& Burrows, C. J. Human NEIL3 Gene Expression Regulated by Epigenetic-Like Oxidative DNA Modification. J. Am. Chem. Soc. 141, 11036-11049 (2019).

440. Fleming, A. M. \& Burrows, C. J. Interplay of Guanine Oxidation and G-Quadruplex Folding in Gene Promoters. Journal of the American Chemical Society 142, 1115-1136 (2020).

441. Zhou, J., Fleming, A. M., Averill, A. M., Burrows, C. J. \& Wallace, S. S. The NEIL glycosylases remove oxidized guanine lesions from telomeric and promoter quadruplex DNA structures. Nucleic Acids Res. 43, 4039-4054 (2015).

442. Konvalinová, H. et al. Diverse effects of naturally occurring base lesions on the structure and stability of the human telomere DNA quadruplex. Biochimie 118, 15-25 (2015).

443. Bielskute, S., Plavec, J. \& Podbevšek, P. Impact of Oxidative Lesions on the Human Telomeric G-Quadruplex. J. Am. Chem. Soc. 141, 25942603 (2019).

444. Bielskute, S., Plavec, J. \& Podbevšek, P. Oxidative lesions modulate G-quadruplex stability and structure in the human BCL2 promoter. Nucleic Acids Res. 49, 2346-2356 (2021).

445. Takahashi, S. et al. Recovery of the Formation and Function of Oxidized G-Quadruplexes by a Pyrene-Modified Guanine Tract. J. Am. Chem. Soc. 140, 5774-5783 (2018).

446. Cogoi, S. \& Xodo, L. E. G-quadruplex formation within the promoter of the KRAS proto-oncogene and its effect on transcription. Nucleic Acids Res. 34, 2536-2549 (2006).

447. Cogoi, S., Paramasivam, M., Spolaore, B. \& Xodo, L. E. Structural polymorphism within a regulatory element of the human KRAS promoter: Formation of G4-DNA recognized by nuclear proteins. Nucleic Acids Res. 36, 3765-3780 (2008). 


\section{References}

448. Paramasivam, M. et al. Protein hnRNP A1 and its derivative Upl unfold quadruplex DNA in the human KRAS promoter: Implications for transcription. Nucleic Acids Res. 37, 2841-2853 (2009).

449. Balasubramanian, S. \& Neidle, S. G-quadruplex nucleic acids as therapeutic targets. Current Opinion in Chemical Biology 13, 345-353 (2009).

450. Balasubramanian, S., Hurley, L. H. \& Neidle, S. Targeting G-quadruplexes in gene promoters: a novel anticancer strategy? Nat. Rev. Drug Discov. 10, 261-275 (2011).

451. Neidle, S. Quadruplex nucleic acids as targets for anticancer therapeutics. Nat. Rev. Chem. 1, 0041 (2017).

452. Siddiqui-Jain, A., Grand, C. L., Bearss, D. J. \& Hurley, L. H. Direct evidence for a G-quadruplex in a promoter region and its targeting with a small molecule to repress c-MYC transcription. Proc. Natl. Acad. Sci. 99, 11593-11598 (2002).

453. Hanahan, D. \& Weinberg, R. A. The hallmarks of cancer. Cell 100, 57-70 (2000).

454. Hanahan, D. \& Weinberg, R. A. Hallmarks of Cancer: The Next Generation. Cell 144, 646-674 (2011).

455. Fouad, Y. A. \& Aanei, C. Revisiting the hallmarks of cancer. Am. J. Cancer Res. 7, 1016-1036 (2017).

456. Brooks, T. A. \& Hurley, L. H. The role of supercoiling in transcriptional control of MYC and its importance in molecular therapeutics. Nat. Rev. Cancer 9, 849-61 (2009).

457. Pirota, V., Nadai, M., Doria, F. \& Richter, S. N. Naphthalene Diimides as Multimodal G-Quadruplex-Selective Ligands. Molecules 24, 426 (2019).

458. Sanchez-Martin, V., Lopez-Pujante, C., Soriano-Rodriguez, M. \& Garcia-Salcedo, J. A. An Updated Focus on Quadruplex Structures as Potential Therapeutic Targets in Cancer. Int. J. Mol. Sci. 21, 8900 (2020).

459. Kosiol, N., Juranek, S., Brossart, P., Heine, A. \& Paeschke, K. G-quadruplexes: a promising target for cancer therapy. Mol. Cancer 20, 40 (2021).

460. Ambrus, A., Chen, D., Dai, J., Jones, R. A. \& Yang, D. Solution structure of the biologically relevant G-quadruplex element in the human cMYC promoter. Implications for G-quadruplex stabilization. Biochemistry 44, 2048-58 (2005).

461. Mathad, R. I., Hatzakis, E., Dai, J. \& Yang, D. C-MYC promoter G-quadruplex formed at the 5'-end of NHE III 1 element: Insights into biological relevance and parallel-stranded G-quadruplex stability. Nucleic Acids Res. 39, 9023-9033 (2011).

462. Dai, J., Chen, D., Jones, R. A., Hurley, L. H. \& Yang, D. NMR solution structure of the major G-quadruplex structure formed in the human BCL2 promoter region. Nucleic Acids Res. 34, 5133-5144 (2006).

463. Dexheimer, T. S., Sun, D. \& Hurley, L. H. Deconvoluting the structural and drug-recognition complexity of the G-quadruplex-forming region upstream of the bcl-2 P1 promoter. J. Am. Chem. Soc. 128, 5404-5415 (2006).

464. Sengupta, P., Chattopadhyay, S. \& Chatterjee, S. G-Quadruplex surveillance in BCL-2 gene: a promising therapeutic intervention in cancer treatment. Drug Discov. Today 22, 1165-1186 (2017).

465. Ducani, C., Bernardinelli, G., Högberg, B., Keppler, B. K. \& Terenzi, A. Interplay of Three G-Quadruplex Units in the KIT Promoter. J. Am. Chem. Soc. 141, 10205-10213 (2019).

466. Morgan, R. K., Batra, H., Gaerig, V. C., Hockings, J. \& Brooks, T. A. Identification and characterization of a new G-quadruplex forming region within the kRAS promoter as a transcriptional regulator. Biochim. Biophys. Acta - Gene Regul. Mech. 1859, 235-245 (2016).

467. Qin, Y., Rezler, E. M., Gokhale, V., Sun, D. \& Hurley, L. H. Characterization of the G-quadruplexes in the duplex nuclease hypersensitive element of the PDGF-A promoter and modulation of PDGF-A promoter activity by TMPyP4. Nucleic Acids Res. 35, 7698-7713 (2007).

468. Qin, Y. et al. Molecular cloning of the human platelet-derived growth factor receptor beta (PDGFR-beta) promoter and drug targeting of the G-quadruplex-forming region to repress PDGFR-beta expression. Biochemistry 49, 4208-19 (2010).

469. De Armond, R., Wood, S., Sun, D., Hurley, L. H. \& Ebbinghaus, S. W. Evidence for the presence of a guanine quadruplex forming region within a polypurine tract of the hypoxia inducible factor 1alpha promoter. Biochemistry 44, 16341-50 (2005).

470. $\mathrm{Xu}, \mathrm{Y}$. \& Sugiyama, H. Structural and functional characterizations of the G-quartet and i-motif elements in retinoblastoma susceptibility genes (Rb). Nucleic Acids Symp. Ser. (Oxf). 177-178 (2005).

471. $\mathrm{Xu}, \mathrm{Y}$. \& Sugiyama, H. Formation of the G-quadruplex and i-motif structures in retinoblastoma susceptibility genes (Rb). Nucleic Acids Res. 34, 949-954 (2006).

472. Garman, E. F. Rosalind Franklin 1920-1958. Acta Crystallogr. Sect. D, Struct. Biol. 76, 698-701 (2020).

473. Wierstra, I. \& Alves, J. The c-myc promoter: still MysterY and challenge. Adv. Cancer Res. 99, 113-333 (2008).

474. Pelengaris, S., Khan, M. \& Evan, G. c-MYC: more than just a matter of life and death. Nat. Rev. Cancer 2, 764-776 (2002).

475. Adhikary, S. \& Eilers, M. Transcriptional regulation and transformation by Myc proteins. Nat. Rev. Mol. Cell Biol. 6, 635-645 (2005).

476. Meyer, N. \& Penn, L. Z. Reflecting on 25 years with MYC. Nat. Rev. Cancer 8, 976-990 (2008).

477. Dang, C. V. MYC on the Path to Cancer. Cell 149, 22-35 (2012).

478. Chen, H., Liu, H. \& Qing, G. Targeting oncogenic Myc as a strategy for cancer treatment. Signal Transduct. Target. Ther. 3, 5 (2018).

479. Duffy, M. J. \& Crown, J. Drugging "undruggable" genes for cancer treatment: Are we making progress? Int. J. Cancer 148, 8-17 (2021).

480. Nair, S. K. \& Burley, S. K. X-Ray Structures of Myc-Max and Mad-Max Recognizing DNA. Cell 112, 193-205 (2003).

481. Dang, C. V., Reddy, E. P., Shokat, K. M. \& Soucek, L. Drugging the 'undruggable' cancer targets. Nat. Rev. Cancer 17, 502-508 (2017).

482. Molyneux, E. M. et al. Burkitt's lymphoma. Lancet 379, 1234-1244 (2012).

483. Eisenman, R. N. Deconstructing Myc. Genes Dev. 15, 2023-2030 (2001).

484. Levens, D. 'You Don't Muck with MYC'. Genes Cancer 1, 547-554 (2010).

485. Gazin, C. et al. Nucleotide sequence of the human c-myc locus: provocative open reading frame within the first exon. EMBO J. 3, 383-387 (1984).

486. Boles, T. C. \& Hogan, M. E. DNA structure equilibria in the human c-myc gene. Biochemistry 26, 367-376 (1987).

487. Siebenlist, U., Hennighausen, L., Battey, J. \& Leder, P. Chromatin structure and protein binding in the putative regulatory region of the c-myc gene in burkitt lymphoma. Cell 37, 381-391 (1984).

488. Mirkin, S. M. et al. DNA H form requires a homopurine-homopyrimidine mirror repeat. Nature 330, 495-497 (1987).

489. Cooney, M., Czernuszewicz, G., Postel, E., Flint, S. \& Hogan, M. Site-specific oligonucleotide binding represses transcription of the human cmyc gene in vitro. Science 241, 456-459 (1988).

490. Postel, E. H., Mango, S. E. \& Flint, S. J. A nuclease-hypersensitive element of the human c-myc promoter interacts with a transcription initiation factor. Mol. Cell. Biol. 9, 5123-5133 (1989).

491. Kinniburgh, A. J., Firulli, A. B. \& Kolluri, R. DNA triplexes and regulation of the c-myc gene. Gene 149, 93-100 (1994).

492. Olivas, W. M. \& Maher, L. J. I. Competitive Triplex/Quadruplex Equilibria Involving Guanine-Rich Oligonucleotides. Biochemistry 34, 278284 (1995).

493. Simonsson, T., Pecinka, P. \& Kubista, M. DNA tetraplex formation in the control region of c-myc. Nucleic Acids Res. 26, 1167-1172 (1998). 


\section{References}

494.

495.

496

497.

498.

499.

500

501.

502.

503.

504

505.

506.

507.

508.

509.

510

511.

512.

513.

514.

515

516

517.

518.

519.

520.

521

522.

523

524

525

526.

527.

528 .

529.

530.

531.

532

533. Freyer, M. W. et al. Biophysical Studies of the c-MYC NHE III1 Promoter: Model Quadruplex Interactions with a Cationic Porphyrin. Biophys.

Simonsson, T., Pribylova, M. \& Vorlickova, M. A Nuclease Hypersensitive Element in the Human c-myc Promoter Adopts Several Distinct iTetraplex Structures. Biochem. Biophys. Res. Commun. 278, 158-166 (2000).

Wang, G. \& Vasquez, K. M. Naturally occurring H-DNA-forming sequences are mutagenic in mammalian cells. Proc. Natl. Acad. Sci. 101, 13448-13453 (2004).

Belotserkovskii, B. P. et al. A Triplex-forming Sequence from the Human c-MYC Promoter Interferes with DNA Transcription. J. Biol. Chem. 282, 32433-32441 (2007).

del Mundo, I. M. A., Zewail-Foote, M., Kerwin, S. M. \& Vasquez, K. M. Alternative DNA structure formation in the mutagenic human c-MYC promoter. Nucleic Acids Res. 45, 4929-4943 (2017).

Kim, H. G. et al. Inhibition of transcription of the human c-myc protooncogene by intermolecular triplex. Biochemistry 37, 2299-304 (1998). oligonucleotides directed to the c-myc gene. Biochemistry 39, 5126-38 (2000).

McGuffie, E. M., Pacheco, D., Carbone, G. M. R. \& Catapano, C. V. Antigene and antiproliferative effects of a c-myc-targeting phosphorothioate triple helix-forming oligonucleotide in human leukemia cells. Cancer Res. 60, 3790-9 (2000).

Christensen, L. A., Finch, R. A., Booker, A. J. \& Vasquez, K. M. Targeting Oncogenes to Improve Breast Cancer Chemotherapy. Cancer Res. 66, 4089-4094 (2006).

Sedoris, K. C. et al. Genomic c-Myc Quadruplex DNA Selectively Kills Leukemia. Mol. Cancer Ther. 11, 66-76 (2012).

Boulware, S. B. et al. Triplex-forming oligonucleotides targeting c- MYC potentiate the anti-tumor activity of gemcitabine in a mouse model of human cancer. Mol. Carcinog. 53, 744-752 (2014).

Muench, D. et al. Quadruplex-forming oligonucleotide targeted to the VEGF promoter inhibits growth of non-small cell lung cancer cells. PLoS One 14, (2019).

Ponzielli, R., Katz, S., Barsyte-Lovejoy, D. \& Penn, L. Z. Cancer therapeutics: Targeting the dark side of Myc. Eur. J. Cancer 41, 2485-2501 (2005).

Kalkat, M. et al. MYC Deregulation in Primary Human Cancers. Genes (Basel). 8, 151 (2017).

Xu-Monette, Z. Y. et al. Clinical and Biologic Significance of MYC Genetic Mutations in De Novo Diffuse Large B-cell Lymphoma. Clin. Cancer Res. 22, 3593-3605 (2016).

Rangan, A., Fedoroff, O. Y. \& Hurley, L. H. Induction of Duplex to G-quadruplex Transition in the c-myc Promoter Region by a Small Molecule. J. Biol. Chem. 276, 4640-4646 (2001).

Dembska, A., Rzepecka, P. \& Juskowiak, B. Spectroscopic Characterization of i-motif Forming c-myc Derived Sequences Double-Labeled with Pyrene. J. Fluoresc. 23, 807-812 (2013).

Reilly, S. M. et al. Folding and Hydrodynamics of a DNA i-Motif from the c-MYC Promoter Determined by Fluorescent Cytidine Analogs. Biophys. J. 107, 1703-1711 (2014).

Paul, S., Hossain, S. S. \& Samanta, A. Insights into the Folding Pathway of a c-MYC-Promoter-Based i-Motif DNA in Crowded Environments at the Single-Molecule Level. J. Phys. Chem. B 124, 763-770 (2020).

Brooks, T. A. \& Hurley, L. H. Targeting MYC Expression through G-Quadruplexes. Genes Cancer 1, 641-649 (2010).

Liu, J. \& Levens, D. Making myc. Curr. Top. Microbiol. Immunol. 302, 1-32 (2006).

Michelotti, E. F., Tomonaga, T., Krutzsch, H. \& Levens, D. Cellular Nucleic Acid Binding Protein Regulates the CT Element of the Human cmyc Protooncogene. J. Biol. Chem. 270, 9494-9499 (1995).

Michelotti, E. F., Michelotti, G. A., Aronsohn, A. I. \& Levens, D. Heterogeneous nuclear ribonucleoprotein Kis a transcription factor. Mol. Cell. Biol. 16, 2350-2360 (1996).

Tomonaga, T. \& Levens, D. Activating transcription from single stranded DNA. Proc. Natl. Acad. Sci. 93, 5830-5835 (1996).

Postel, E., Berberich, S., Flint, S. \& Ferrone, C. Human c-myc transcription factor PuF identified as nm23-H2 nucleoside diphosphate kinase, a candidate suppressor of tumor metastasis. Science 261, 478-480 (1993).

Berberich, S. J. \& Postel, E. H. PuF/NM23-H2/NDPK-B transactivates a human c-myc promoter-CAT gene via a functional nuclease hypersensitive element. Oncogene 10, 2343-7 (1995).

Ji, L., Arcinas, M. \& Boxer, L. M. The Transcription Factor, Nm23H2, Binds to and Activates the Translocated c-myc Allele in Burkitt's Lymphoma. J. Biol. Chem. 270, 13392-13398 (1995).

Rigo, R., Palumbo, M. \& Sissi, C. G-quadruplexes in human promoters: A challenge for therapeutic applications. Biochim. Biophys. Acta - Gen. Subj. 1861, 1399-1413 (2017).

Sun, D. \& Hurley, L. H. The Importance of Negative Superhelicity in Inducing the Formation of G-Quadruplex and i-Motif Structures in the c-Myc Promoter: Implications for Drug Targeting and Control of Gene Expression. J. Med. Chem. 52, 2863-2874 (2009).

Stump, S., Mou, T.-C., Sprang, S. R., Natale, N. R. \& Beall, H. D. Crystal structure of the major quadruplex formed in the promoter region of the human c-MYC oncogene. PLoS One 13, e0205584 (2018).

Dettler, J. M., Buscaglia, R., Le, V. H. \& Lewis, E. A. DSC Deconvolution of the Structural Complexity of c-MYC P1 Promoter G-Quadruplexes. Biophys. J. 100, 1517-1525 (2011).

Marchand, A., Rosu, F., Zenobi, R. \& Gabelica, V. Thermal Denaturation of DNA G-Quadruplexes and Their Complexes with Ligands: Thermodynamic Analysis of the Multiple States Revealed by Mass Spectrometry. J. Am. Chem. Soc. 140, 12553-12565 (2018).

Prochownik, E. V. \& Vogt, P. K. Therapeutic Targeting of Myc. Genes Cancer 1, 650-659 (2010).

Koh, C. M., Sabò, A. \& Guccione, E. Targeting MYC in cancer therapy: RNA processing offers new opportunities. BioEssays 38, 266-275 (2016).

Allen-Petersen, B. L. \& Sears, R. C. Mission Possible: Advances in MYC Therapeutic Targeting in Cancer. BioDrugs 33, 539-553 (2019).

Wolf, E. \& Eilers, M. Targeting MYC Proteins for Tumor Therapy. Annu. Rev. Cancer Biol. 4, 61-75 (2020).

Ross, J. et al. Targeting MYC: From understanding its biology to drug discovery. Eur. J. Med. Chem. 213, 113137 (2021).

Wang, W. et al. Human MYC G-quadruplex: From discovery to a cancer therapeutic target. Biochimica et Biophysica Acta - Reviews on Cancer 1874, 188410 (2020).

Liu, W. et al. Structures of 1:1 and 2:1 complexes of BMVC and MYC promoter G-quadruplex reveal a mechanism of ligand conformation adjustment for G4-recognition. Nucleic Acids Res. 47, 11931-11942 (2019).

Asamitsu, S., Obata, S., Yu, Z., Bando, T. \& Sugiyama, H. Recent Progress of Targeted G-Quadruplex-Preferred Ligands Toward Cancer Therapy. Molecules 24, 429 (2019). J. 92, 2007-2015 (2007). 


\section{References}

534. Głuszyńska, A., Juskowiak, B., Kuta-Siejkowska, M., Hoffmann, M. \& Haider, S. Carbazole ligands as c-myc G-quadruplex binders. Int. J. Biol. Macromol. 114, 479-490 (2018).

535. Felsenstein, K. M. et al. Small Molecule Microarrays Enable the Identification of a Selective, Quadruplex-Binding Inhibitor of MYC Expression. ACS Chem. Biol. 11, 138-148 (2016).

536. Pany, S. P. P., Bommisetti, P., Diveshkumar, K. V. \& Pradeepkumar, P. I. Benzothiazole hydrazones of furylbenzamides preferentially stabilize c-MYC and c-KIT1 promoter G-quadruplex DNAs. Org. Biomol. Chem. 14, 5779-5793 (2016).

537. Wu, T.-Y., Huang, Q., Huang, Z.-S., Hu, M.-H. \& Tan, J.-H. A drug-like imidazole-benzothiazole conjugate inhibits malignant melanoma by stabilizing the c-MYC G-quadruplex. Bioorg. Chem. 99, 103866 (2020).

538. Dutta, D. et al. Cell penetrating thiazole peptides inhibit c-MYC expression via site-specific targeting of c-MYC G-quadruplex. Nucleic Acids Res. 46, 5355-5365 (2018).

539. Panda, D., Saha, P., Das, T. \& Dash, J. Target guided synthesis using DNA nano-templates for selectively assembling a G-quadruplex binding c-MYC inhibitor. Nat. Commun. 8, 16103 (2017).

540. Nimbarte, V. D. et al. Synthesis and In vitro Evaluation of novel 5-nitroIndole Derivatives as c-Myc G-Quadruplex Binders with Anticancer Activities. ChemMedChem cmdc.202000835 (2021). doi:10.1002/cmdc.202000835

541. Chauhan, A. et al. Synthesis of Fluorescent Binaphthyl Amines That Bind c-MYC G-Quadruplex DNA and Repress c-MYC Expression. J. Med. Chem. 59, 7275-7281 (2016).

542. Pavan Kumar, Y. et al. Fluorescent Dansyl-Guanosine Conjugates that Bind $c-M Y C$ Promoter G-Quadruplex and Downregulate $c-M Y C$ Expression. ChemBioChem 17, 388-393 (2016).

543. Hu, M. H. et al. Discovery of a New Four-Leaf Clover-Like Ligand as a Potent c-MYC Transcription Inhibitor Specifically Targeting the Promoter G-Quadruplex. J. Med. Chem. 61, 2447-2459 (2018).

544. Asamitsu, S. et al. Simultaneous Binding of Hybrid Molecules Constructed with Dual DNA-Binding Components to a G-Quadruplex and Its Proximal Duplex. Chem. Eur. J. 24, 4428-4435 (2018).

545. Asamitsu, S., Li, Y., Bando, T. \& Sugiyama, H. Ligand-Mediated G-Quadruplex Induction in a Double-Stranded DNA Context by Cyclic Imidazole/Lysine Polyamide. ChemBioChem 17, 1317-1322 (2016).

546. Binas, O. et al. $19 \mathrm{~F}$ NMR-Based Fragment Screening for 14 Different Biologically Active RNAs and 10 DNA and Protein Counter-Screens. ChemBioChem 22, 423-433 (2021).

547. Drygin, D. et al. Anticancer activity of CX-3543: A direct inhibitor of rRNA biogenesis. Cancer Res. 69, 7653-7661 (2009).

548. Xu, H. et al. CX-5461 is a DNA G-quadruplex stabilizer with selective lethality in BRCA1/2 deficient tumours. Nat. Commun. 8, 14432 (2017).

549. Brown, R. V., Danford, F. L., Gokhale, V., Hurley, L. H. \& Brooks, T. A. Demonstration that drug-targeted down-regulation of MYC in nonHodgkins lymphoma is directly mediated through the promoter G-quadruplex. J. Biol. Chem. 286, 41018-27 (2011).

550. Das, T., Panda, D., Saha, P. \& Dash, J. Small Molecule Driven Stabilization of Promoter G-Quadruplexes and Transcriptional Regulation of cMYC. Bioconjug. Chem. 29, 2636-2645 (2018).

551. Calabrese, D. R. et al. Chemical and structural studies provide a mechanistic basis for recognition of the MYC G-quadruplex. Nat. Commun. 9, 4229 (2018).

552. Bouvard, C. et al. Small molecule selectively suppresses MYC transcription in cancer cells. Proc. Natl. Acad. Sci. 114, 3497-3502 (2017).

553. Musso, L. et al. c-MYC G-quadruplex binding by the RNA polymerase I inhibitor BMH-21 and analogues revealed by a combined NMR and biochemical Approach. Biochim. Biophys. Acta - Gen. Subj. 1862, 615-629 (2018).

554. Cercek, A., Wheler, J., Murray, P. E., Zhou, S. \& Saltz, L. Phase 1 study of APTO-253 HCl, an inducer of KLF4, in patients with advanced or metastatic solid tumors. Invest. New Drugs 33, 1086-1092 (2015).

555. Local, A. et al. APTO-253 stabilizes G-quadruplex DNA, inhibits MYC expression, and induces DNA damage in acute myeloid leukemia cells. Mol. Cancer Ther. 17, 1177-1186 (2018).

556. Tsai, C. Y. et al. APTO-253 is a new addition to the repertoire of drugs that can exploit DNA BRCA1/2 Deficiency. Mol. Cancer Ther. 17, 11671176 (2018).

557. Pal, S. \& Paul, S. An in silico investigation of the binding modes and pathway of APTO-253 on c-KIT G-quadruplex DNA. Phys. Chem. Chem. Phys. 23, 3361-3376 (2021).

558. Haddach, M. et al. Discovery of CX-5461, the first direct and selective inhibitor of RNA polymerase I, for cancer therapeutics. ACS Med. Chem. Lett. 3, 602-606 (2012).

559. Lee, H. C. et al. RNA Polymerase I Inhibition with CX-5461 as a Novel Therapeutic Strategy to Target MYC in Multiple Myeloma. Br. J. Haematol. 177, 80-94 (2017).

560. Mars, J.-C. et al. The chemotherapeutic agent CX-5461 irreversibly blocks RNA polymerase I initiation and promoter release to cause nucleolar disruption, DNA damage and cell inviability. NAR Cancer 2, (2020).

561. Yan, S. et al. The RNA polymerase I transcription inhibitor CX-5461 cooperates with topoisomerase 1 inhibition by enhancing the DNA damage response in homologous recombination-proficient high-grade serous ovarian cancer. Br. J. Cancer 124, 616-627 (2021).

562. Bruno, P. M. et al. The primary mechanism of cytotoxicity of the chemotherapeutic agent CX-5461 is topoisomerase II poisoning. Proc. Natl. Acad. Sci. U. S. A. 117, 4053-4060 (2020).

563. Sanij, E. et al. CX-5461 activates the DNA damage response and demonstrates therapeutic efficacy in high-grade serous ovarian cancer. Nat. Commun. 11, 1-18 (2020).

564. Sullivan, H.J., Chen, B. \& Wu, C. Molecular dynamics study on the binding of an anticancer DNA G-quadruplex stabilizer, CX-5461, to human telomeric, C-Kit1, and c-Myc G-quadruplexes and a DNA duplex. J. Chem. Inf. Model. 60, 5203-5224 (2020).

565. Khot, A. et al. First-in-human RNA polymerase I transcription inhibitor CX-5461 in patients with advanced hematologic cancers: Results of a phase I dose-escalation study. Cancer Discov. 9, 1036-1049 (2019).

566. Jana, J. et al. Human cathelicidin peptide LL37 binds telomeric G-quadruplex. Mol. Biosyst. 9, 1833-1836 (2013).

567. Sengupta, P. et al. Site-specific amino acid substitution in dodecameric peptides determines the stability and unfolding of c-MYC quadruplex promoting apoptosis in cancer cells. Nucleic Acids Res. 46, 9932-9950 (2018).

568. Purcell, E. M. Research in nuclear magnetism. Nobel Lecture December 11 (1952). Available at: https://www.nobelprize.org/uploads/2018/06/purcell-lecture.pdf. (Accessed: 19th January 2021)

569. Binas, O., Bessi, I. \& Schwalbe, H. Structure Validation of G-Rich RNAs in Noncoding Regions of the Human Genome. ChemBioChem 21, 1656-1663 (2020).

570. Tunis-Schneider, M. J. B. \& Maestre, M. F. Circular dichroism spectra of oriented and unoriented deoxyribonucleic acid films-A preliminary study. J. Mol. Biol. 52, 521-541 (1970). 


\section{References}

571. Del Villar-Guerra, R., Trent, J. O. \& Chaires, J. B. G-Quadruplex Secondary Structure Obtained from Circular Dichroism Spectroscopy. Angew. Chem. Int. Ed. 57, 7171-7175 (2018).

572. Kypr, J., Kejnovska, I., Renciuk, D. \& Vorlickova, M. Circular dichroism and conformational polymorphism of DNA. Nucleic Acids Res. 37, $1713-1725$ (2009).

573. Gray, D. M. et al. Measured and calculated CD spectra of G-quartets stacked with the same or opposite polarities. Chirality 20, 431-440 (2008).

574. Vorličková, M., Kejnovská, I., Bednářová, K., Renčiuk, D. \& Kypr, J. Circular Dichroism Spectroscopy of DNA: From Duplexes to Quadruplexes. Chirality 24, 691-698 (2012).

575. Daly, S., Rosu, F. \& Gabelica, V. Mass-resolved electronic circular dichroism ion spectroscopy. Science 368, 1465-1468 (2020).

576. Overhauser, A. W. Polarization of Nuclei in Metals. Phys. Rev. 92, 411-415 (1953).

577. Carver, T. R. \& Slichter, C. P. Polarization of Nuclear Spins in Metals. Phys. Rev. 92, 212-213 (1953).

578. Solomon, I. Relaxation Processes in a System of Two Spins. Phys. Rev. 99, 559-565 (1955).

579. Jeener, J., Meier, B. H., Bachmann, P. \& Ernst, R. R. Investigation of exchange processes by two-dimensional NMR spectroscopy. J. Chem. Phys. 71, 4546-4553 (1979).

580. Kumar, A., Ernst, R. R. \& Wüthrich, K. A two-dimensional nuclear Overhauser enhancement (2D NOE) experiment for the elucidation of complete proton-proton cross-relaxation networks in biological macromolecules. Biochem. Biophys. Res. Commun. 95, 1-6 (1980).

581. Vögeli, B. The nuclear Overhauser effect from a quantitative perspective. Prog. Nucl. Magn. Reson. Spectrosc. 78, 1-46 (2014).

582. Fürtig, B., Richter, C., Wöhnert, J. \& Schwalbe, H. NMR spectroscopy of RNA. ChemBioChem 4, 936-962 (2003).

583. Schnieders, R., Keyhani, S., Schwalbe, H. \& Fürtig, B. More than Proton Detection - New Avenues for NMR Spectroscopy of RNA. Chem.A Eur.J. 26, 102-113 (2020).

584. Schnieders, R. et al. NMR Spectroscopy of Large Functional RNAs: From Sample Preparation to Low-Gamma Detection. Curr. Protoc. nucleic acid Chem. 82, e116 (2020).

585. Webba da Silva, M. NMR methods for studying quadruplex nucleic acids. Methods 43, 264-277 (2007).

586. Feigon, J., Koshlap, K. M. \& Smith, F. W. 1H NMR spectroscopy of DNA triplexes and quadruplexes. Methods Enzymol. 261, 225-55 (1995).

587. Zimmer, D. P. \& Crothers, D. M. NMR of enzymatically synthesized uniformly $13 \mathrm{C} 15 \mathrm{~N}$-labeled DNA oligonucleotides. Proc. Natl. Acad. Sci. U. S. A. 92, 3091-5 (1995).

588. Louis, J. M., Martin, R. G., Clore, G. M. \& Gronenborn, A. M. Preparation of Uniformly Isotope-labeled DNA Oligonucleotides for NMR Spectroscopy. J. Biol. Chem. 273, 2374-2378 (1998).

589. Lohmann, J. S., Stougaard, M. \& Koch, J. A new enzymatic route for production of long 5'-phosphorylated oligonucleotides using suicide cassettes and rolling circle DNA synthesis. BMC Biotechnol. 7, 49 (2007).

590. Nelissen, F. H. T., Girard, F. C., Tessari, M., Heus, H. A. \& Wijmenga, S. S. Preparation of selective and segmentally labeled single-stranded DNA for NMR by self-primed PCR and asymmetrical endonuclease double digestion. Nucleic Acids Res. 37, e114 (2009).

591. Nelissen, F. H. T., Goossens, E. P. M., Tessari, M. \& Heus, H. A. Enzymatic preparation of multimilligram amounts of pure single-stranded DNA samples for material and analytical sciences. Anal. Biochem. 475, 68-73 (2015).

592. Nelissen, F. H. T., Tessari, M., Wijmenga, S. S. \& Heus, H. A. Stable isotope labeling methods for DNA. Prog. Nucl. Magn. Reson. Spectrosc. 96, 89-108 (2016).

593. Nunes, A. R. D., Chavante, S. F., Rocha, H. A. O. \& Lanza, D. C. F. 'in-House' Production of Single Stranded Oligodeoxyribonucleotides. Nucleic Acid Ther. 27, 115-120 (2017).

594. Meija, J. et al. Isotopic compositions of the elements 2013 (IUPAC Technical Report). Pure Appl. Chem. 88, 293-306 (2016).

595. Phan, A. T. Long-range imino proton-13C J-couplings and the through-bond correlation of imino and non-exchangeable protons in unlabeled DNA. J. Biomol. NMR 16, 175-178 (2000).

596. Phan, A. T. Through-bond correlation of sugar and base protons in unlabeled nucleic acids. J. Magn. Reson. 153, 223-6 (2001).

597. Adrian, M., Winnerdy, F. R., Heddi, B. \& Phan, A. T. Rotation of Guanine Amino Groups in G-Quadruplexes: A Probe for Local Structure and Ligand Binding. Biophys. J. 113, 775-784 (2017).

598. Schnieders, R. et al. Novel 13C-detected NMR Experiments for the Precise Detection of RNA Structure. Angew. Chem. Int. Ed. 58, 9140-9144 (2019).

599. Bothe, J. R. et al. Characterizing RNA dynamics at atomic resolution using solution-state NMR spectroscopy. Nat. Methods 8, 919-931 (2011). 600. Al-Hashimi, H. M. NMR studies of nucleic acid dynamics. J. Magn. Reson. 237, 191-204 (2013).

601. Zhao, B. \& Zhang, Q. Characterizing excited conformational states of RNA by NMR spectroscopy. Curr. Opin. Struct. Biol. 30, 134-146 (2015).

602. Rinnenthal, J. et al. Mapping the Landscape of RNA Dynamics with NMR Spectroscopy. Acc. Chem. Res. 44, 1292-1301 (2011).

603. Palmer, A. G. NMR Characterization of the Dynamics of Biomacromolecules. Chem. Rev. 104, 3623-3640 (2004).

604. Markwick, P. R. L., Malliavin, T. \& Nilges, M. Structural Biology by NMR: Structure, Dynamics, and Interactions. PLoS Comput. Biol. 4, e1000168 (2008).

605. Marušič, M., Schlagnitweit, J. \& Petzold, K. RNA Dynamics by NMR Spectroscopy. ChemBioChem 20, 2685-2710 (2019).

606. Gáspári, Z. \& Perczel, A. Protein Dynamics as Reported by NMR. in Annual Reports on NMR Spectroscopy 35-75 (2010). doi:10.1016/B978$0-08-089054-8.00002-2$

607. Reich, H. NMR spectroscopy. Chem 605, University of Wisconsin - Madison chapter 8, Relaxation in NMR spectroscopy (2020). Available at: https://organicchemistrydata.org/hansreich/resources/nmr. (Accessed: 4th March 2021)

608. Bloembergen, N., Purcell, E. M. \& Pound, R. V. Relaxation Effects in Nuclear Magnetic Resonance Absorption. Phys. Rev. 73, 679-712 (1948).

609. Levitt, M. H. Spin dynamics: basics of nuclear magnetic resonance. John Wiley Sons, LTD 1. edition, chapter 16 Relaxation, pp. 513-537, ISBN: $04714892(2001)$.

610. Lipari, G. \& Szabo, A. Model-free approach to the interpretation of nuclear magnetic resonance relaxation in macromolecules. 1. Theory and range of validity. J. Am. Chem. Soc. 104, 4546-4559 (1982).

611. Akke, M., Fiala, R., Jiang, F., Patel, D. \& Palmer, A. G. Base dynamics in a UUCG tetraloop RNA hairpin characterized by $15 \mathrm{~N}$ spin relaxation: correlations with structure and stability. RNA 3, 702-9 (1997).

612. Shajani, Z. \& Varani, G. 13C NMR relaxation studies of RNA base and ribose nuclei reveal a complex pattern of motions in the RNA binding site for human U1A protein. J. Mol. Biol. 349, 699-715 (2005).

613. Ban, D., Sabo, T., Griesinger, C. \& Lee, D. Measuring Dynamic and Kinetic Information in the Previously Inaccessible Supra-tc Window of Nanoseconds to Microseconds by Solution NMR Spectroscopy. Molecules 18, 11904-11937 (2013).

614. Tolman, J. R. \& Ruan, K. NMR residual dipolar couplings as probes of biomolecular dynamics. Chem. Rev. 106, 1720-36 (2006). 


\section{References}

615. Zhang, Q. \& Al-Hashimi, H. M. Domain-elongation NMR spectroscopy yields new insights into RNA dynamics and adaptive recognition. RNA 15, 1941-1948 (2009).

616. Chiarparin, E., Rüdisser, S. \& Bodenhausen, G. Hydrogen Bonds in RNA Base Pairs Investigated by Cross-Correlated Relaxation of MultipleQuantum Coherence in NMR. ChemPhysChem 2, 41-45 (2001).

617. Vögeli, B. Comprehensive description of NMR cross-correlated relaxation under anisotropic molecular tumbling and correlated local dynamics on all time scales. J. Chem. Phys. 133, 014501 (2010).

618. Brutscher, B. Principles and applications of cross-correlated relaxation in biomolecules. Concepts Magn. Reson. 12, 207-229 (2000).

619. Carr, H. Y. \& Purcell, E. M. Effects of Diffusion on Free Precession in Nuclear Magnetic Resonance Experiments. Phys. Rev. 94, 630-638 (1954).

620. Meiboom, S. \& Gill, D. Modified Spin-Echo Method for Measuring Nuclear Relaxation Times. Rev. Sci. Instrum. 29, 688-691 (1958).

621. Juen, M. A. et al. Excited States of Nucleic Acids Probed by Proton Relaxation Dispersion NMR Spectroscopy. Angew. Chem. Int. Ed. 55, 12008$12(2016)$.

622. Forsén, S. \& Hoffman, R. A. Study of Moderately Rapid Chemical Exchange Reactions by Means of Nuclear Magnetic Double Resonance. J. Chem. Phys. 39, 2892-2901 (1963).

623. Vallurupalli, P., Sekhar, A., Yuwen, T. \& Kay, L. E. Probing conformational dynamics in biomolecules via chemical exchange saturation transfer: a primer. J. Biomol. NMR 67, 243-271 (2017).

624. Wenter, P., Bodenhausen, G., Dittmer, J. \& Pitsch, S. Kinetics of RNA refolding in dynamic equilibrium by $1 \mathrm{H}$-detected 15N exchange NMR spectroscopy. J. Am. Chem. Soc. 128, 7579-87 (2006).

625. Kloiber, K., Spitzer, R., Tollinger, M., Konrat, R. \& Kreutz, C. Probing RNA dynamics via longitudinal exchange and CPMG relaxation dispersion NMR spectroscopy using a sensitive 13C-methyl label. Nucleic Acids Res. 39, 4340-51 (2011).

626. Van Nuland, N. A. J., Forge, V., Balbach, J. \& Dobson, C. M. Real-Time NMR Studies of Protein Folding. Acc. Chem. Res. 31, 773-780 (1998).

627. Fürtig, B. et al. Time-resolved NMR studies of RNA folding. Biopolymers 86, 360-383 (2007).

628. Pintér, G. et al. Real-time NMR spectroscopy in the study of biomolecular kinetics and dynamics. Magn. Reson. preprint (2021). doi: $10.5194 / \mathrm{mr}-2021-16$

629. Rennella, E. \& Brutscher, B. Fast Real-Time NMR Methods for Characterizing Short-Lived Molecular States. ChemPhysChem 14, 3059-3070 (2013).

630. Favier, A. \& Brutscher, B. Recovering lost magnetization: polarization enhancement in biomolecular NMR. J. Biomol. NMR 49, 9-15 (2011).

631. Schanda, P. \& Brutscher, B. Very fast two-dimensional NMR spectroscopy for real-time investigation of dynamic events in proteins on the time scale of seconds. J. Am. Chem. Soc. (2005). doi:10.1021/ja051306e

632. Schanda, P., Kupče, E. \& Brutscher, B. SOFAST-HMQC Experiments for Recording Two-dimensional Deteronuclear Correlation Spectra of Proteins within a Few Seconds. J. Biomol. NMR 33, 199-211 (2005).

633. Schanda, P., Van Melckebeke, H. \& Brutscher, B. Speeding Up Three-Dimensional Protein NMR Experiments to a Few Minutes. J. Am. Chem. Soc. 128, 9042-9043 (2006).

634. Gouilleux, B., Rouger, L. \& Giraudeau, P. Ultrafast 2D NMR: Methods and Applications. in Annual Reports on NMR Spectroscopy 75-144 (2018). doi:10.1016/bs.arnmr.2017.08.003

635. Giraudeau, P. \& Frydman, L. Ultrafast 2D NMR: An Emerging Tool in Analytical Spectroscopy. Annu. Rev. Anal. Chem. 7, 129-161 (2014).

636. Harris, T., Szekely, O. \& Frydman, L. On the Potential of Hyperpolarized Water in Biomolecular NMR Studies. J. Phys. Chem. B 118, 32813290 (2014).

637. Olsen, G., Markhasin, E., Szekely, O., Bretschneider, C. \& Frydman, L. Optimizing water hyperpolarization and dissolution for sensitivityenhanced 2D biomolecular NMR. J. Magn. Reson. 264, 49-58 (2016).

638. Szekely, O., Olsen, G. L., Felli, I. C. \& Frydman, L. High-Resolution 2D NMR of Disordered Proteins Enhanced by Hyperpolarized Water. Anal. Chem. 90, 6169-6177 (2018).

639. Szekely, O., Olsen, G. L., Novakovic, M., Rosenzweig, R. \& Frydman, L. Assessing Site-Specific Enhancements Imparted by Hyperpolarized Water in Folded and Unfolded Proteins by 2D HMQC NMR. J. Am. Chem. Soc. 142, 9267-9284 (2020).

640. Olsen, G. L. et al. Sensitivity-enhanced three-dimensional and carbon-detected two-dimensional NMR of proteins using hyperpolarized water. J. Biomol. NMR 74, 161-171 (2020).

641. Novakovic, M. et al. A 300-fold enhancement of imino nucleic acid resonances by hyperpolarized water provides a new window for probing RNA refolding by 1D and 2D NMR. Proc. Natl. Acad. Sci. U. S. A. 117, 2449-2455 (2020).

642. Mishkovsky, M. \& Frydman, L. Progress in Hyperpolarized Ultrafast 2D NMR Spectroscopy. ChemPhysChem 9, 2340-2348 (2008).

643. Lee, M.-K., Gal, M., Frydman, L. \& Varani, G. Real-time multidimensional NMR follows RNA folding with second resolution. Proc. Natl. Acad. Sci. 107, 9192-9197 (2010).

644. Gal, M., Mishkovsky, M. \& Frydman, L. Real-Time Monitoring of Chemical Transformations by Ultrafast 2D NMR Spectroscopy. J.Am. Chem. Soc. 128, 951-956 (2006).

645. Dethoff, E. A., Petzold, K., Chugh, J., Casiano-Negroni, A. \& Al-Hashimi, H. M. Visualizing transient low-populated structures of RNA. Nature (2012). doi:10.1038/nature11498

646. Kimsey, I. J. et al. Dynamic basis for dG•dT misincorporation via tautomerization and ionization. Nature 554, 195-201 (2018).

647. Schuler, B. \& Eaton, W. A. Protein folding studied by single-molecule FRET. Curr. Opin. Struct. Biol. 18, 16-26 (2008).

648. Yoo, J., Louis, J. M., Gopich, I. V. \& Chung, H. S. Three-Color Single-Molecule FRET and Fluorescence Lifetime Analysis of Fast Protein Folding. J. Phys. Chem. B 122, 11702-11720 (2018).

649. Wirth, A. J., Liu, Y., Prigozhin, M. B., Schulten, K. \& Gruebele, M. Comparing Fast Pressure Jump and Temperature Jump Protein Folding Experiments and Simulations. J. Am. Chem. Soc 137, 7152-7159 (2015).

650. Jung, A. High-temperature solution NMR structure of TmCsp. Protein Sci. 13, 342-350 (2004).

651. Kachel, N., Kremer, W., Zahn, R. \& Kalbitzer, H. R. Observation of intermediate states of the human prion protein by high pressure NMR spectroscopy. BMC Struct. Biol. 6, 16 (2006).

652. Dreydoppel, M. et al. Equilibrium and Kinetic Unfolding of GB1: Stabilization of the Native State by Pressure. J. Phys. Chem. B 122, 88468852 (2018).

653. Cavini, I. A. et al. Inhibition of amyloid A $\beta$ aggregation by high pressures or specific d-enantiomeric peptides. Chem. Commun. (Camb). 54, 3294-3297 (2018).

654. Kamatari, Y. O., Kitahara, R., Yamada, H., Yokoyama, S. \& Akasaka, K. High-pressure NMR spectroscopy for characterizing folding intermediates and denatured states of proteins. Methods 34, 133-43 (2004).

655. Kalbitzer, H. R. High pressure NMR methods for characterizing functional substates of proteins. Subcell. Biochem. 72, 179-197 (2015). 


\section{References}

656. Akasaka, K. High pressure NMR spectroscopy. Subcell. Biochem. 72, 707-721 (2015).

657. Caro, J. A. \& Wand, A. J. Practical aspects of high-pressure NMR spectroscopy and its applications in protein biophysics and structural biology. Methods 148, 67-80 (2018).

658. Baldwin, A. J. \& Kay, L. E. NMR spectroscopy brings invisible protein states into focus. Nat. Chem. Biol. 5, 808-814 (2009).

659. Roche, J., Ying, J., Maltsev, A. S. \& Bax, A. Impact of Hydrostatic Pressure on an Intrinsically Disordered Protein: A High-Pressure NMR Study of $\alpha$-Synuclein. ChemBioChem 14, 1754-1761 (2013).

660. Kalbitzer, H. R. et al. Intrinsic allosteric inhibition of signaling proteins by targeting rare interaction states detected by high-pressure NMR spectroscopy. Angew. Chem. Int. Ed. 52, 14242-6 (2013).

661. Naito, A., Nakatani, H., Imanari, M. \& Akasaka, K. State-correlated two-dimensional NMR spectroscopy. J. Magn. Reson. 87, 429-432 (1990).

662. Kawakami, M. \& Akasaka, K. Microwave temperature-jump nuclear magnetic resonance system for aqueous solutions. Rev. Sci. Instrum. 69, 3365-3369 (1998).

663. Ferguson, D. B., Krawietz, T. R. \& Haw, J. F. Temperature-Jump MAS NMR with a Laser Heater. J. Magn. Reson. Ser. A 109, 273-275 (1994).

664. Mildner, T., Ernst, H., Freude, D. \& Hölderich, W. F. Temperature-Jump Relaxation Magic-Angle Spinning NMR Study of the Methyl tertButyl Ether Reaction in a Boron Pentasil Zeolite. J. Am. Chem. Soc. 119, 4258-4262 (1997).

665. Yushmanov, P. V. \& Furó, I. A temperature-jump design for conventional NMR probes. J. Magn. Reson. 181, 148-153 (2006).

666. Rinnenthal, J. et al. A temperature-jump NMR probe setup using rf heating optimized for the analysis of temperature-induced biomacromolecular kinetic processes. J. Magn. Reson. 251, 84-93 (2015).

667. Gal, M., Zibzener, K. \& Frydman, L. A capacitively coupled temperature-jump arrangement for high-resolution biomolecular NMR. Magn. Reson. Chem. 48, 842-847 (2010).

668. Reinhard Arnold, M., Robert Kalbitzer, H. \& Kremer, W. High-sensitivity sapphire cells for high pressure NMR spectroscopy on proteins. J. Magn. Reson. 161, 127-131 (2003).

669. Charlier, C. et al. Study of protein folding under native conditions by rapidly switching the hydrostatic pressure inside an NMR sample cell. Proc. Natl. Acad. Sci. 115, E4169-E4178 (2018).

670. Heuert, U., Krumova, M., Hempel, G., Schiewek, M. \& Blume, A. NMR probe for pressure-jump experiments up to 250 bars and 3 ms jump time. Rev. Sci. Instrum. 81, 105102 (2010).

671. Kremer, W. et al. Pulsed Pressure Perturbations, an Extra Dimension in NMR Spectroscopy of Proteins. J. Am. Chem. Soc. 133, 13646-13651 (2011).

672. Wu, W.-J., Vidugiris, G., Mooberry, E. S., Westler, W. M. \& Markley, J. L. Mixing apparatus for preparing NMR samples under pressure. J. Magn. Reson. 164, 84-91 (2003).

673. Peterson, R. W. \& Wand, A. J. Self-contained high-pressure cell, apparatus, and procedure for the preparation of encapsulated proteins dissolved in low viscosity fluids for nuclear magnetic resonance spectroscopy. Rev. Sci. Instrum. 76, 094101 (2005).

674. Akasaka, K., Naito, A. \& Nakatani, H. Temperature-jump NMR study of protein folding: Ribonuclease A at low pH. J. Biomol. NMR 1, 65-70 (1991).

675. Pintér, G. \& Schwalbe, H. Refolding of Cold-Denatured Barstar Induced by Radio-Frequency Heating: A New Method to Study Protein Folding by Real-Time NMR Spectroscopy. Angew. Chem. Int. Ed. 59, 22086-22091 (2020).

676. Alderson, T. R., Charlier, C., Torchia, D. A., Anfinrud, P. \& Bax, A. Monitoring Hydrogen Exchange During Protein Folding by Fast Pressure Jump NMR Spectroscopy. J. Am. Chem. Soc 139, (2017).

677. Charlier, C., Courtney, J. M., Anfinrud, P. \& Bax, A. Interrupted Pressure-Jump NMR Experiments Reveal Resonances of On-Pathway Protein Folding Intermediate. J. Phys. Chem. B 122, 11792-11799 (2018).

678. Barnes, C. A., Robertson, A. J., Louis, J. M., Anfinrud, P. \& Bax, A. Observation of $\beta$-Amyloid Peptide Oligomerization by Pressure-Jump NMR Spectroscopy. J. Am. Chem. Soc. 141, 13762-13766 (2019).

679. Kamatari, Y. O., Yokoyama, S., Tachibana, H. \& Akasaka, K. Pressure-jump NMR Study of Dissociation and Association of Amyloid Protofibrils. J. Mol. Biol. 349, 916-921 (2005).

680. Munte, C. E., Beck Erlach, M., Kremer, W., Koehler, J. \& Kalbitzer, H. R. Distinct conformational states of the Alzheimer $\beta$-amyloid peptide can be detected by high-pressure NMR spectroscopy. Angew. Chem. Int. Ed. 52, 8943-7 (2013).

681. RODER, H. Rapid mixing methods for exploring the kinetics of protein folding. Methods 34, 15-27 (2004).

682. Yushmanov, P. V. \& Furó, I. A rapid-mixing design for conventional NMR probes. J. Magn. Reson. 175, 264-270 (2005).

683. Mok, K. H. et al. Rapid sample-mixing technique for transient NMR and photo-CIDNP spectroscopy: Applications to real-time protein folding. J. Am. Chem. Soc. 125, 12484-12492 (2003).

684. Franco, R., Favier, A., Schanda, P. \& Brutscher, B. Optimized fast mixing device for real-time NMR applications. J. Magn. Reson. 281, 125-129 (2017).

685. Balbach, J. et al. Following protein folding in real time using NMR spectroscopy. Nat. Struct. Biol. 2, 865-870 (1995).

686. Kiefhaber, T., Labhardt, A. M. \& Baldwin, R. L. Direct NMR evidence for an intermediate preceding the rate-limiting step in the unfolding of ribonuclease A. Nature 375, 513-515 (1995).

687. Balbach, J. et al. Protein Folding Monitored at Individual Residues During a Two-Dimensional NMR Experiment. Science 274, 1161-1163 (1996).

688. Corazza, A. et al. Native-unlike long-lived intermediates along the folding pathway of the amyloidogenic protein $\beta 2$-microglobulin revealed by real-time two-dimensional NMR. J. Biol. Chem. 285, 5827-5835 (2010).

689. ZEEB, M. Protein folding studied by real-time NMR spectroscopy. Methods 34, 65-74 (2004).

690. Dobson, C. M. \& Hore, P. J. Kinetic studies of protein folding using NMR spectroscopy. Nat. Struct. Biol. 5, 504-507 (1998).

691. Schanda, P., Forge, V. \& Brutscher, B. Protein folding and unfolding studied at atomic resolution by fast two-dimensional NMR spectroscopy. Proc. Natl. Acad. Sci. 104, 11257-11262 (2007).

692. Reining, A. et al. Three-state mechanism couples ligand and temperature sensing in riboswitches. Nature 499, 355-9 (2013).

693. Manoharan, V., Fürtig, B., Jäschke, A. \& Schwalbe, H. Metal-induced folding of diels-alderase ribozymes studied by static and time-resolved NMR spectroscopy. J. Am. Chem. Soc. 131, 6261-6270 (2009).

694. Barraud, P. et al. Time-resolved NMR monitoring of tRNA maturation. Nat. Commun. 10, 3373 (2019).

695. Buck, J., Furtig, B., Noeske, J., Wohnert, J. \& Schwalbe, H. Time-resolved NMR methods resolving ligand-induced RNA folding at atomic resolution. Proc. Natl. Acad. Sci. 104, 15699-15704 (2007).

696. Kühn, T. \& Schwalbe, H. Monitoring the kinetics of ion-dependent protein folding by time-resolved NMR spectroscopy at atomic resolution. J. Am. Chem. Soc. 122, 6169-6174 (2000). 


\section{References}

697. Wirmer, J., Kühn, T. \& Schwalbe, H. Millisecond Time Resolved Photo-CIDNP NMR Reveals a Non-Native Folding Intermediate on the IonInduced Refolding Pathway of Bovine $\alpha$-Lactalbumin. Angew. Chem. Int. Ed. 40, 4248-4251 (2001).

698. Mayer, G. \& Heckel, A. Biologically active molecules with a 'light switch'. Angew. Chem. Int. Ed. 45, 4900-21 (2006).

699. Brieke, C., Rohrbach, F., Gottschalk, A., Mayer, G. \& Heckel, A. Light-Controlled Tools. Angew. Chem. Int. Ed. 51, 8446-8476 (2012).

700. Becker, Y. et al. A red-shifted two-photon-only caging group for three-dimensional photorelease. Chem. Sci. 9, 2797-2802 (2018).

701. Becker, Y. et al. Selective Modification for Red-Shifted Excitability: A Small Change in Structure, a Huge Change in Photochemistry. Chem.A Eur. J. 27, 2212-2218 (2021).

702. Griffiths, J. II. Photochemistry of azobenzene and its derivatives. Chem. Soc. Rev. 1, 481 (1972).

703. Yamana, K., Yoshikawa, A. \& Nakano, H. Synthesis of a new photoisomerizable linker for connecting two oligonucleotide segments. Tetrahedron Lett. 37, 637-640 (1996).

704. Asanuma, H., Ito, T. \& Komiyama, M. Photo-responsive oligonucleotides carrying azobenzene in the side-chains. Tetrahedron Lett. 39, 90159018 (1998).

705. Asanuma, Ito, Yoshida, Liang \& Komiyama. Photoregulation of the Formation and Dissociation of a DNA Duplex by Using the cis-trans Isomerization of Azobenzene. Angew. Chem. Int. Ed. 38, 2393-2395 (1999).

706. Barltrop, J. A. \& Schofield, P. Photosensitive Protecting Groups. Tetrahedron Lett. 3, 697-699 (1962).

707. Engels, J. \& Schlaeger, E. J. Synthesis, Structure, and Reactivity of Adenosine Cyclic 3',5'-Phosphate-Benzyl Triesters. J. Med. Chem. 20, $907-$ $911(1977)$.

708. Monroe, W. T., McQuain, M. M., Chang, M. S., Alexander, J. S. \& Haselton, F. R. Targeting Expression with Light Using Caged DNA. J. Biol. Chem. 274, 20895-20900 (1999).

709. Ando, H., Furuta, T., Tsien, R. Y. \& Okamoto, H. Photo-mediated gene activation using caged RNA/DNA in zebrafish embryos. Nat. Genet. 28, 317-325 (2001).

710. Shah, S., Rangarajan, S. \& Friedman, S. H. Light-activated RNA interference. Angew. Chem. Int. Ed. 44, 1328-32 (2005).

711. Ting, R., Lermer, L. \& Perrin, D. M. Triggering DNAzymes with Light: A Photoactive C8 Thioether-Linked Adenosine. J. Am. Chem. Soc. 126, 12720-12721 (2004).

712. Wenter, P., Fürtig, B., Hainard, A., Schwalbe, H. \& Pitsch, S. Kinetics of photoinduced RNA refolding by real-time NMR spectroscopy. Angew. Chem. Int. Ed. 44, 2600-3 (2005).

713. Höbartner, C. \& Silverman, S. K. Modulation of RNA tertiary folding by incorporation of caged nucleotides. Angew. Chem. Int. Ed. 44, 73059 (2005).

714. Heckel, A. et al. An Anticoagulant with Light-Triggered Antidote Activity. Angew. Chem. Int. Ed. 45, 6748-6750 (2006).

715. Kröck, L. \& Heckel, A. Photoinduced transcription by using temporarily mismatched caged oligonucleotides. Angew. Chem. Int. Ed. 44, 471-3 (2005).

716. Mayer, G., Kröck, L., Mikat, V., Engeser, M. \& Heckel, A. Light-Induced Formation of G-Quadruplex DNA Secondary Structures. ChemBioChem 6, 1966-1970 (2005).

717. Chaulk, S. Caged RNA: photo-control of a ribozyme reaction. Nucleic Acids Res. 26, 3173-3178 (1998).

718. Chaulk, S. G. \& MacMillan, A. M. Synthesis of oligo-RNAs with photocaged adenosine 2'-hydroxyls. Nat. Protoc. 2, 1052-1058 (2007).

719. Zheng, G., Cochella, L., Liu, J., Hobert, O. \& Li, W. Temporal and Spatial Regulation of MicroRNA Activity with Photoactivatable Cantimirs. ACS Chem. Biol. 6, 1332-1338 (2011).

720. Griepenburg, J. C., Ruble, B. K. \& Dmochowski, I. J. Caged oligonucleotides for bidirectional photomodulation of let-7 miRNA in zebrafish embryos. Bioorg. Med. Chem. 21, 6198-6204 (2013).

721. Seyfried, P., Eiden, L., Grebenovsky, N., Mayer, G. \& Heckel, A. Photo-Tethers for the (Multi-)Cyclic, Conformational Caging of Long Oligonucleotides. Angew. Chem. Int. Ed. 56, 359-363 (2017).

722. Kaplan, J. H., Forbush, B. \& Hoffman, J. F. Rapid photolytic release of adenosine 5'-triphosphate from a protected analog: utilization by the sodium:potassium pump of human red blood cell ghosts. Biochemistry 17, 1929-1935 (1978).

723. Wenter, P., Fürtig, B., Hainard, A., Schwalbe, H. \& Pitsch, S. A Caged Uridine for the Selective Preparation of an RNA Fold and Determination of its Refolding Kinetics by Real-Time NMR. ChemBioChem 7, 417-420 (2006).

724. Rodrigues-Correia, A. et al. Comparison of the duplex-destabilizing effects of nucleobase-caged oligonucleotides. Anal. Bioanal. Chem. 399, 441-447 (2011).

725. Steinert, H. S., Schäfer, F., Jonker, H. R. A., Heckel, A. \& Schwalbe, H. Influence of the absolute configuration of npe-caged cytosine on DNA single base pair stability. Angew. Chem. Int. Ed. 53, 1072-5 (2014).

726. Seyfried, P. et al. Optimal Destabilization of DNA Double Strands by Single-Nucleobase Caging. Chem. Eur. J. 24, 17568-17576 (2018).

727. Fürtig, B. et al. Conformational dynamics of bistable RNAs studied by time-resolved NMR spectroscopy. J. Am. Chem. Soc. 129, 16222-16229 (2007).

728. Fürtig, B., Wenter, P., Pitsch, S. \& Schwalbe, H. Probing Mechanism and Transition State of RNA Refolding. ACS Chem. Biol. 5, 753-765 (2010).

729. Cao, S., Fürtig, B., Schwalbe, H. \& Chen, S.-J. Folding Kinetics for the Conformational Switch between Alternative RNA Structures. J. Phys. Chem. B 114, 13609-13615 (2010).

730. Höbartner, C., Ebert, M.-O., Jaun, B. \& Micura, R. RNA Two-State Conformation Equilibria and the Effect of Nucleobase Methylation. Angew. Chem. Int. Ed. 41, 605-609 (2002).

731. Höbartner, C., Mittendorfer, H., Breuker, K. \& Micura, R. Triggering of RNA secondary structures by a functionalized nucleobase. Angew. Chem. Int. Ed. 43, 3922-5 (2004).

732. Grün, J. T. et al. Conformational Dynamics of Strand Register Shifts in DNA G-Quadruplexes. J. Am. Chem. Soc. 142, 264-273 (2020).

733. Grün, J. T. et al. Unraveling the Kinetics of Spare-Tire DNA G-Quadruplex Folding. J. Am. Chem. Soc. accepted manuscript, (published online) (2021). doi:https://doi.org/10.1021/jacs.1c01089

734. Georg, I. et al. Exhaustively Trichlorosilylated C 1 and C 2 Building Blocks: Beyond the Müller-Rochow Direct Process. J. Am. Chem. Soc. 140, 9696-9708 (2018).

735. Georg, I. et al. Building up Strain in One Step: Synthesis of an Edge-Fused Double Silacyclobutene from an Extensively Trichlorosilylated Butadiene Dianion. Angew. Chem. Int. Ed. 59, 16181-16187 (2020).

736. Helmling, C. et al. Life times of metastable states guide regulatory signaling in transcriptional riboswitches. Nat. Commun. 9, 944 (2018).

737. Harkness V, R. W., Avakyan, N., Sleiman, H. F. \& Mittermaier, A. K. Mapping the energy landscapes of supramolecular assembly by thermal hysteresis. Nat. Commun. 9, 3152 (2018). 


\section{References}

738. Wang, Z., Chen, R., Hou, L., Li, J. \& Liu, J.-P. Molecular dynamics and principal components of potassium binding with human telomeric intramolecular G-quadruplex. Protein Cell 6, 423-433 (2015).

739. Balthasart, F., Plavec, J. \& Gabelica, V. Ammonium ion binding to DNA G-quadruplexes: do electrospray mass spectra faithfully reflect the solution-phase species? J. Am. Soc. Mass Spectrom. 24, 1-8 (2013).

740. Hud, N. V., Schultze, P. \& Feigon, J. Ammonium Ion as an NMR Probe for Monovalent Cation Coordination Sites of DNA Quadruplexes. J. Am. Chem. Soc. 120, 6403-6404 (1998).

741. Hud, N. V., Schultze, P., Sklenár, V. \& Feigon, J. Binding sites and dynamics of ammonium ions in a telomere repeat DNA quadruplex. J. Mol. Biol. 285, 233-43 (1999).

742. Šket, P., Črnugelj, M. \& Plavec, J. d(G3T4G4) forms unusual dimeric G-quadruplex structure with the same general fold in the presence of $\mathrm{K}+, \mathrm{Na}+$ or NH4+ ions. Bioorg. Med. Chem. 12, 5735-5744 (2004).

743. Sket, P., Crnugelj, M., Kozminski, W. \& Plavec, J. 15NH4+ ion movement inside d(G4T4G4)2 G-quadruplex is accelerated in the presence of smaller Na+ ions. Org. Biomol. Chem. 2, 1970-3 (2004).

744. Sket, P. \& Plavec, J. Not all G-quadruplexes exhibit ion-channel-like properties: NMR study of ammonium ion (non)movement within the d(G(3)T(4)G(4))(2) quadruplex. J. Am. Chem. Soc. 129, 8794-800 (2007).

745. Podbevsek, P., Sket, P. \& Plavec, J. Stacking and not solely topology of T3 loops controls rigidity and ammonium ion movement within d(G4T3G4)2 G-quadruplex. J. Am. Chem. Soc. 130, 14287-93 (2008).

746. Trajkovski, M., Šket, P. \& Plavec, J. Cation localization and movement within DNA thrombin binding aptamer in solution. Org. Biomol. Chem. 7, 4677 (2009).

747. Trajkovski, M. \& Plavec, J. Assessing Roles of Cations in G-Quadruplex-Based Nanowires by NMR. J. Phys. Chem. C 116, 23821-23825 (2012).

748. Wong, A., Ida, R. \& Wu, G. Direct NMR detection of the "invisible” alkali metal cations tightly bound to G-quadruplex structures. Biochem. Biophys. Res. Commun. 337, 363-366 (2005).

749. Ida, R. \& Wu, G. Direct NMR Detection of Alkali Metal Ions Bound to G-Quadruplex DNA. J. Am. Chem. Soc. 130, 3590-3602 (2008).

750. Fiala, R. et al. NMR Cross-Correlated Relaxation Rates Reveal Ion Coordination Sites in DNA. J. Am. Chem. Soc. 133, 13790-13793 (2011).

751. Sket, P. Identification of mixed di-cation forms of G-quadruplex in solution. Nucleic Acids Res. 33, 3691-3697 (2005).

752. Harkness, R. W. et al. Parallel reaction pathways accelerate folding of a guanine quadruplex. Nucleic Acids Res. 49, 1247-1262 (2021).

753. Alberty, R. A. Principle of Detailed Balance in Kinetics. J. Chem. Educ. 81, 1206 (2004).

754. Wright, C. F., Lindorff-Larsen, K., Randles, L. G. \& Clarke, J. Parallel protein-unfolding pathways revealed and mapped. Nat. Struct. Mol. Biol. 10, 658-662 (2003).

755. Dobson, C. M., Šali, A. \& Karplus, M. Protein Folding: A Perspective from Theory and Experiment. Angew. Chem. Int. Ed. 37, 868-893 (1998).

756. Baldwin, R. L. The nature of protein folding pathways: the classical versus the new view. J. Biomol. NMR 5, 103-9 (1995).

757. Wildegger, G. \& Kiefhaber, T. Three-state model for lysozyme folding: triangular folding mechanism with an energetically trapped intermediate. J. Mol. Biol. 270, 294-304 (1997).

758. Dinner, A. R., Šali, A., Smith, L. J., Dobson, C. M. \& Karplus, M. Understanding protein folding via free-energy surfaces from theory and experiment. Trends Biochem. Sci. 25, 331-339 (2000).

759. Zaidi, F. N., Nath, U. \& Udgaonkar, J. B. Multiple intermediates and transition states during protein unfolding. Nat. Struct. Biol. 4, 1016-1024 (1997).

760. Bonnet, G., Krichevsky, O. \& Libchaber, A. Kinetics of conformational fluctuations in DNA hairpin-loops. Proc. Natl. Acad. Sci. 95, 8602-8606 (1998).

761. Ansari, A., Kuznetsov, S. V. \& Shen, Y. Configurational diffusion down a folding funnel describes the dynamics of DNA hairpins. Proc. Natl. Acad. Sci. 98, 7771-7776 (2001).

762. Wallace, M. I., Ying, L., Balasubramanian, S. \& Klenerman, D. Non-Arrhenius kinetics for the loop closure of a DNA hairpin. Proc. Natl. Acad. Sci. 98, 5584-5589 (2001).

763. Zhang, W. \& Chen, S.-J. Exploring the Complex Folding Kinetics of RNA Hairpins: I. General Folding Kinetics Analysis. Biophys. J. 90, 765777 (2006)

764. Zhang, W. \& Chen, S.-J. Exploring the Complex Folding Kinetics of RNA Hairpins: II. Effect of Sequence, Length, and Misfolded States. Biophys. J. 90, 778-787 (2006).

765. Kuznetsov, S. V., Ren, C.-C., Woodson, S. A. \& Ansari, A. Loop dependence of the stability and dynamics of nucleic acid hairpins. Nucleic Acids Res. 36, 1098-1112 (2007).

766. Chen, S.-J. RNA Folding: Conformational Statistics, Folding Kinetics, and Ion Electrostatics. Annu. Rev. Biophys. 37, 197-214 (2008).

767. Črnugelj, M., Šket, P. \& Plavec, J. Small Change in a G-Rich Sequence, a Dramatic Change in Topology: New Dimeric G-Quadruplex Folding Motif with Unique Loop Orientations. J. Am. Chem. Soc. 125, 7866-7871 (2003).

768. Novakovic, M. et al. Sensitivity enhancement of homonuclear multidimensional NMR correlations for labile sites in proteins, polysaccharides, and nucleic acids. Nat. Commun. 11, 5317 (2020). 


\section{Appendix}

\subsection{HMBC and Deuterium Exchange NMR Spectrum}

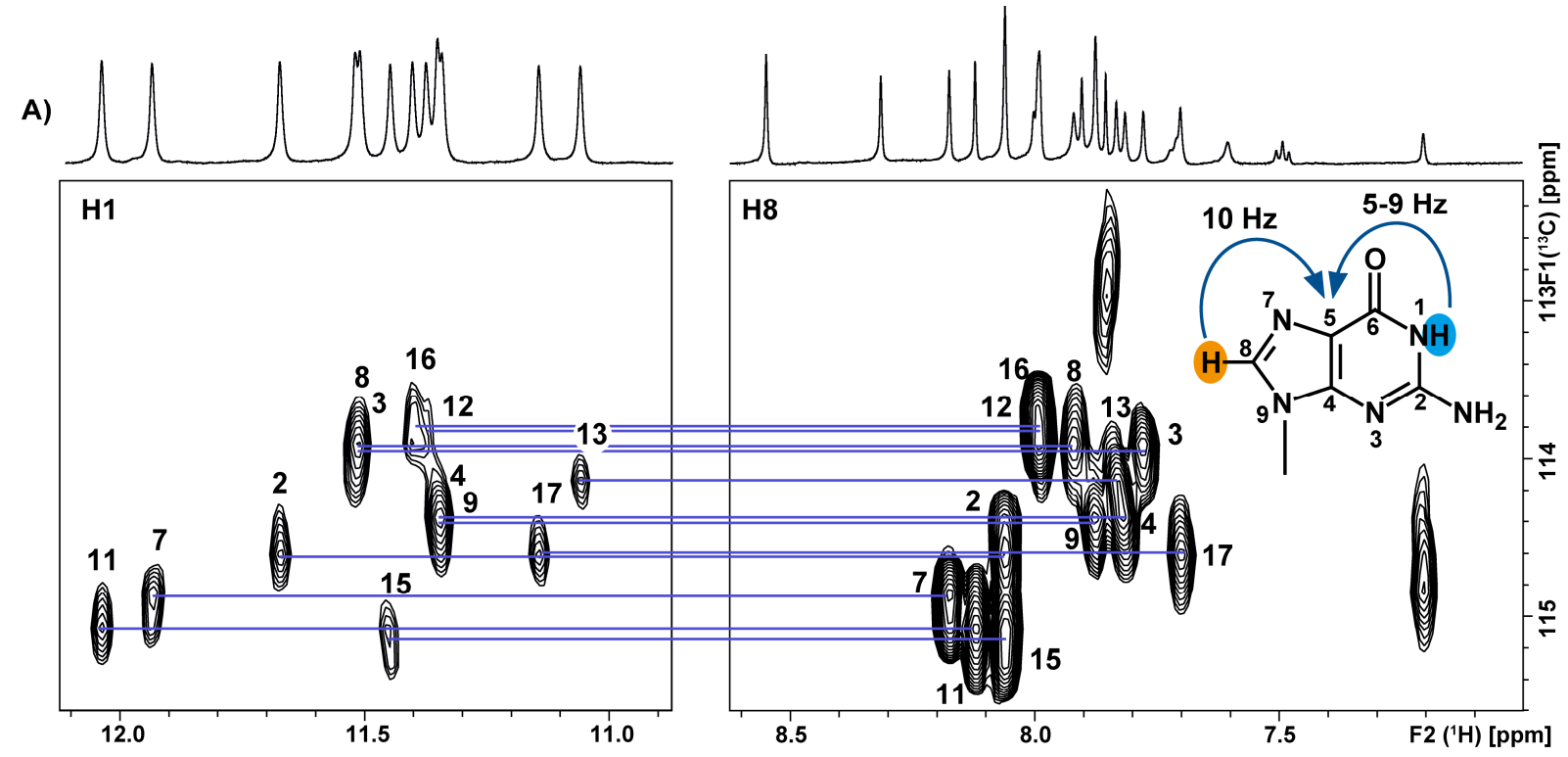

B)

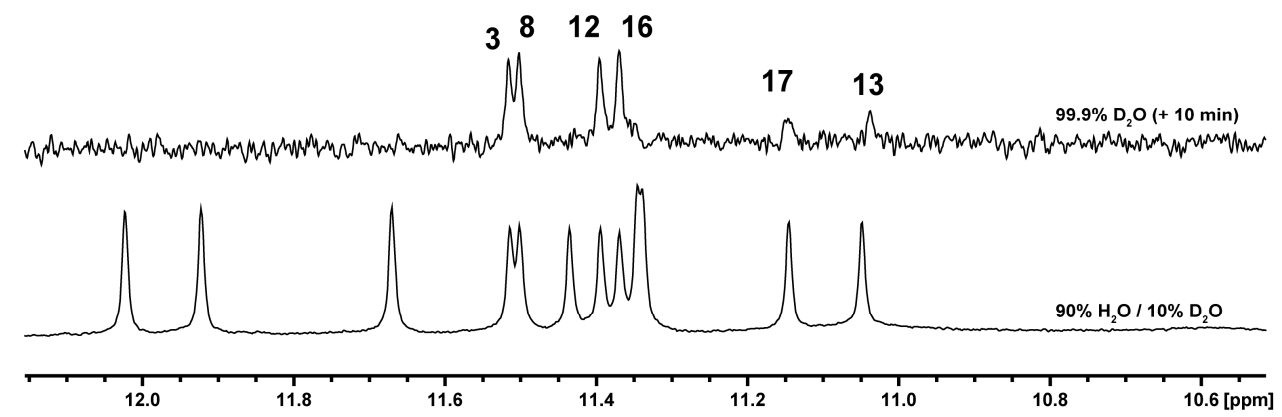

Figure 66: Representative NMR spectra of photocaged $c M Y C-2345-53$ 18-mer for H1-H8 correlations and hydrogendeuterium exchange. A) $500 \mu \mathrm{M}$ DNA, 90/10 H $\mathrm{H}_{2} \mathrm{O} / \mathrm{D}_{2} \mathrm{O}(298 \mathrm{~K}), 600 \mathrm{MHz}(\mathrm{WW}), \mathrm{HMBC}$ (hmbcetgpl3nd.ric) with jump-return-echo water suppression, d19 $=55 \mu \mathrm{s}, \mathrm{J}(\mathrm{XH})=17 \mathrm{~Hz} ; \mathrm{B}) 100 \mu \mathrm{M} \mathrm{DNA}(298 \mathrm{~K}), 700 \mathrm{MHz}$, jump-return echo water suppression (hs1lecho). Referenced to DSS. 


\subsection{Plots of Time-Resolved 1D ${ }^{1} \mathrm{H}$ NMR Spectra for Spare-Tire G4 Folding}

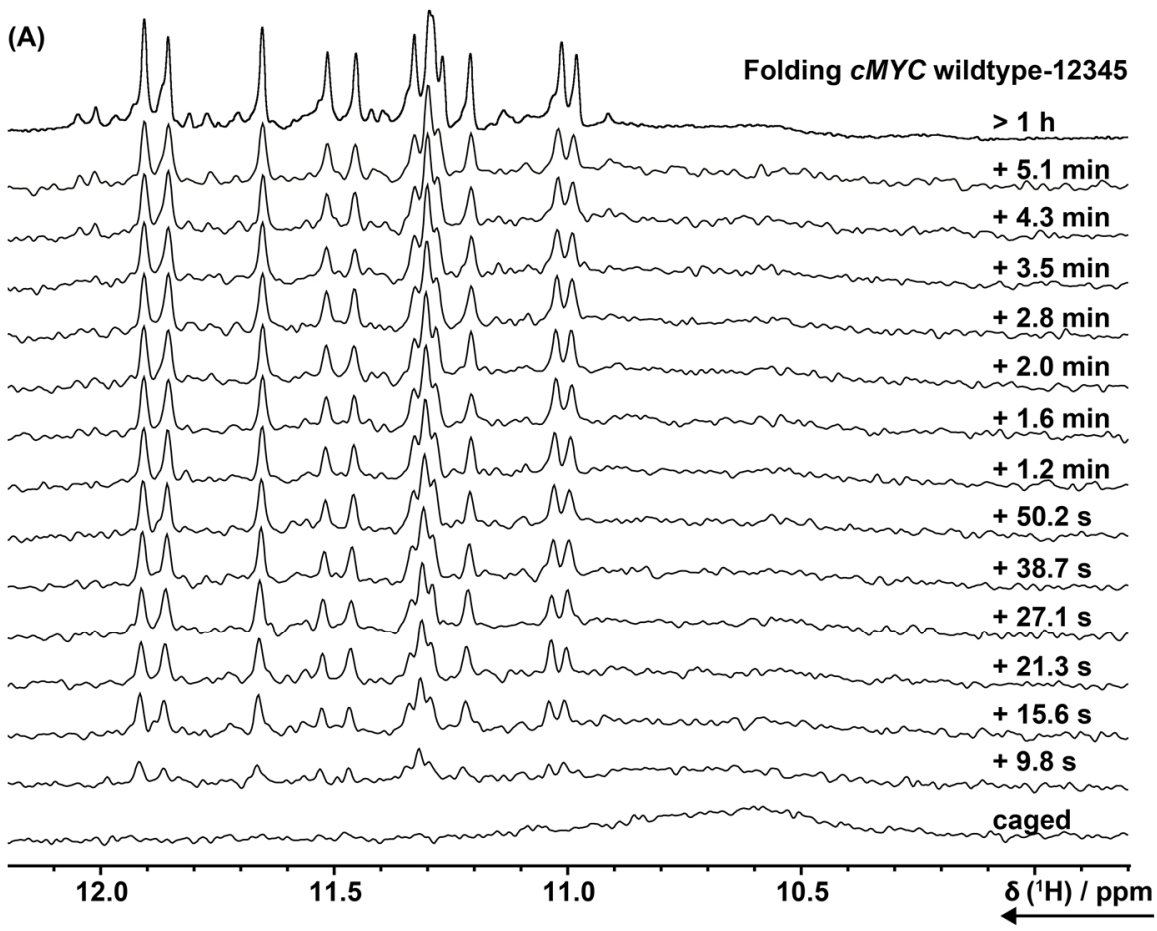

(B)

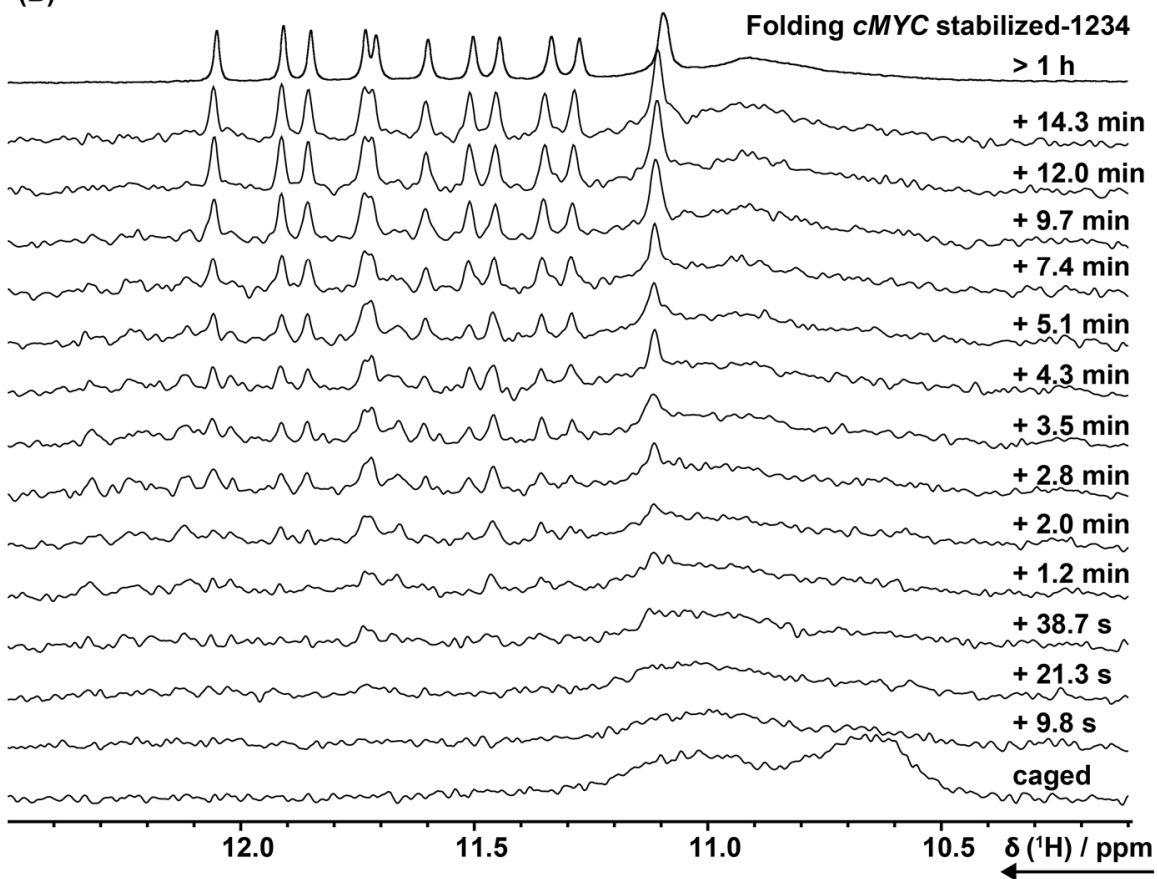

Figure 67: Plots of ${ }^{1} \mathrm{H}$ imino proton regions of time-resolved NMR spectra (700 MHz, jump-return-echo water suppression (hs1lecho, $\mathrm{d} 19=50 \mu \mathrm{s}$ ) at indicated times after light-induced folding initiation. (A) Folding of wt-12345 at $298 \mathrm{~K}$. (B) Folding of stab.-1234 at $285 \mathrm{~K}$. 


\subsection{Python Script for Kinetic Fits}

\begin{tabular}{|c|}
\hline 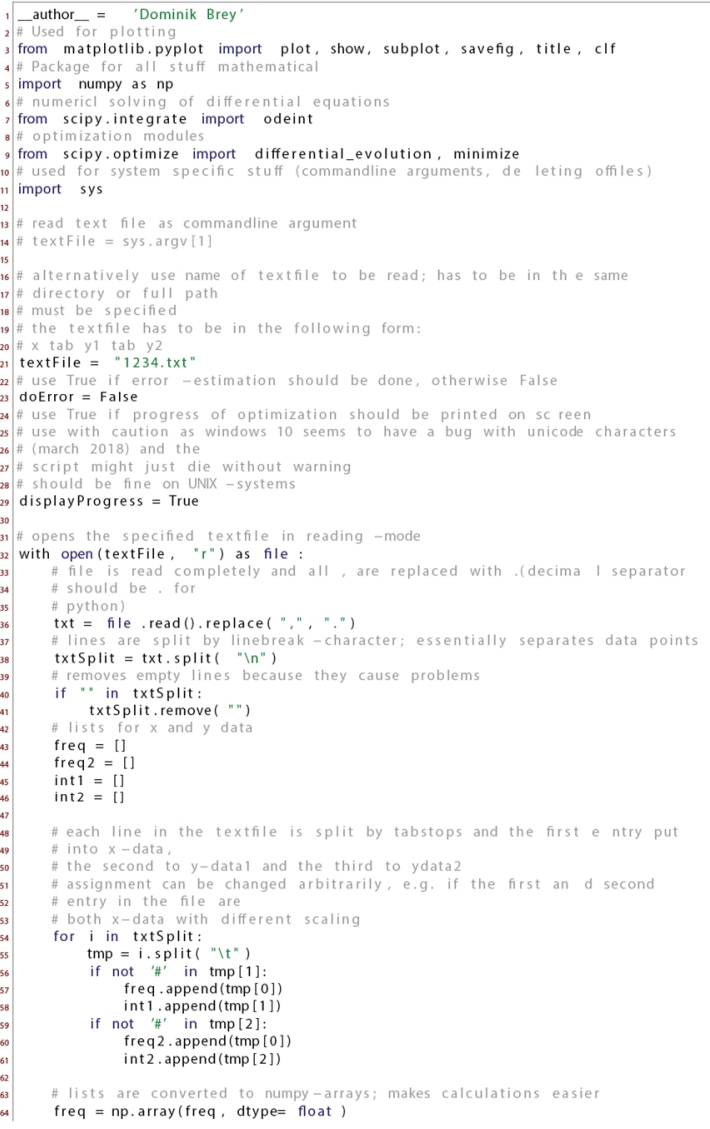 \\
\hline
\end{tabular}

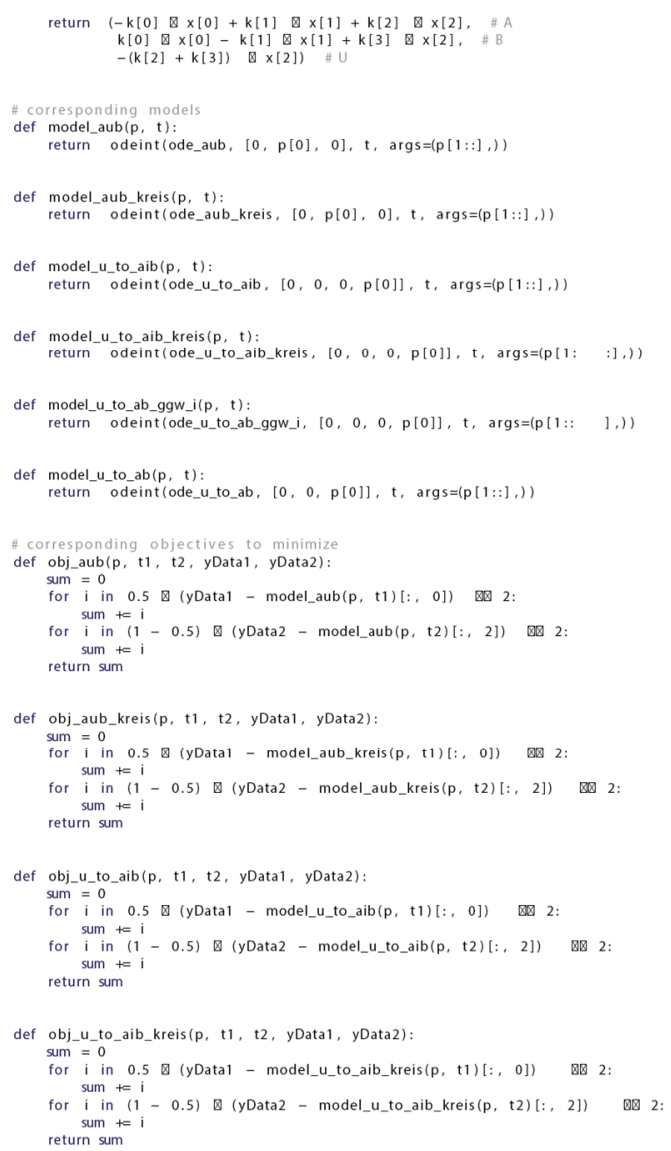

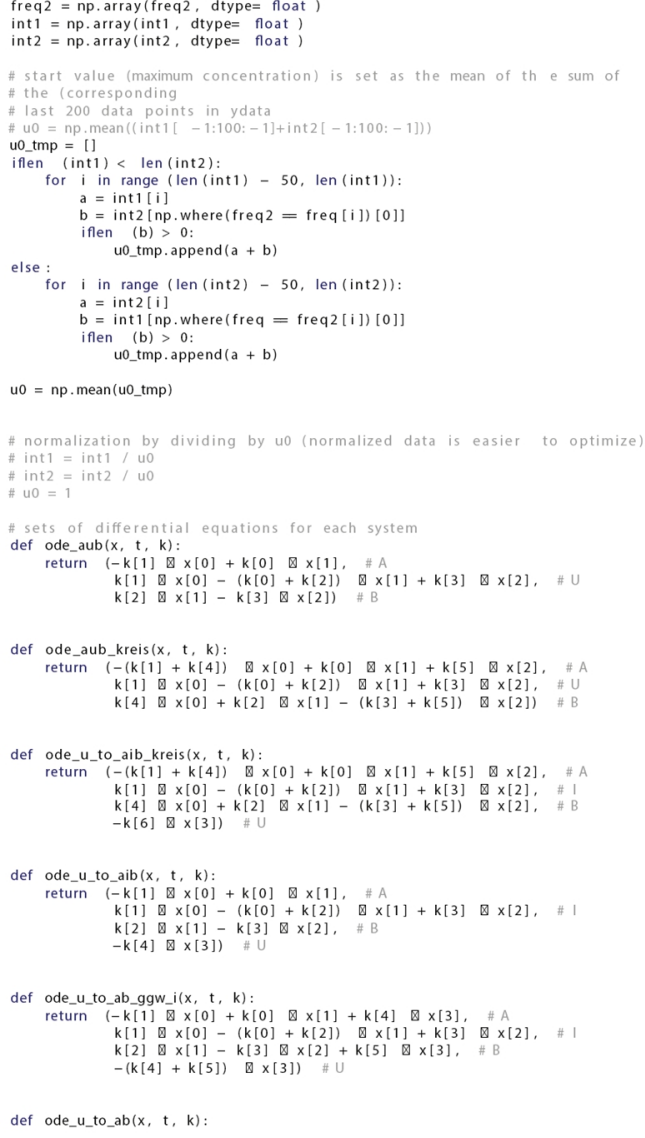

def obj_u_to_ab_ggw_ii (p, t1, t2, yData1, yData2):

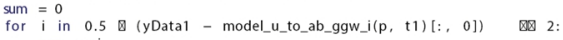

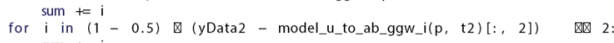
sum $t=\mathrm{i}$
return sum

def obj_u_to_ab $(p, t 1, t 2, y D a t a 1, y D a t a 2)$ :

for $i$ in 0.5 区 (yDatal - model_u_to_ab $(p, t)$ [ $1:, 0]$ ) 四 2

for $i$ in $(1-0.5)$ Q $($ yData2 - model_u_to_ab $(p, t 2)[:, 1])$ 㫬 2 bounds=bounds_aub_kreis, popsize $=2$, print ('Success in global optimization of system aub kreis',

res_aub_kreis. success, ' $(n '$ '
k_opt_aub_kreis_diff = res_aub_kreis.x

print ('Start Optimization of system 4 to aib' 's)

bounds_u_to_aib $=($
(uo $\square 0.95$, uo $₫ 1.05),(5,100),(5,100),(5,100),(0.01,10)$, res_u_to_aib = differential_evolution (obj_u_to_aib. args =(freq, freq2, int 1, int 2, ), bounds=bounds_u_to_aib, popsize $=2$,

print ('Success in global optimization of system u_to aib:"

res_u_to_aib. success, ('In')
k_opt_u_to__aib_diff = res_u_to_aib.x

print ('Start Optimization of system u_to_aib_kreis' )

(u0 00.95, uo $\square 1.05),(5,500),(5,500),(0.01,50),(0.01,50)$ $(0.1,10),(0.01,10)$

res_u_to_aib_kreis = differential_evolution (obj_u_to_ aib_kreis,

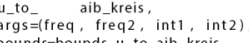
a
bounds=bounds_u_to_aib_kreis, popsize $=2$,
disp $=$ displayprogress ) 
print ('Success in global optimization of system u_to_aib_kreis: res_u_to_ab_kreis. success,

k_opt_u_to_aib_kreis_diff = res_u_to_aib_kreis $x$

print ('Start Optimization of system U_to_ab_ggw_i'

(uo $₫ 0.99$, u 00
$(0.01,1000)$

$(0.01,1000),(0.01,1000)$.

_u to ab ggw $1=$

args $=$ (freq, freq 2 , int 1, int 2,$)$
bounds=bounds_u_to_ab_ggw $i$, opsize $=2$,

print ('Success in global optimization of system u_to_ab_ggw_i: ',

res_u_to_ab_ggw_i. success,

k_opt_u_to_ab_ggw_i_diff = res_u_to_ab_ggw_i.x

print ('Start Optimization of system u_to_ab'

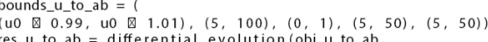
args =(freq, freq2, int 1, int 2,$)$ bounds=bounds_u_to_ab.

disp=displayprogress)
print ('Success in global optimization of system $u$ to ab:

res_u_to_ab. success, ' ( $\mid \mathrm{n}$ ')

"error approximation by bootstrap algorithm
if doError:

print ('Start approximating standard errorin' )

newDatafits_aub $=$ [1

print ('Standard error for system aub: ',

for $\mathrm{i}$ in range $(0,100)$

newXData1 $=$

newYVata1 $=1$

the

smallest $1=\max ($ int 1$)$

ame size as the original sample

for index in range $(0$, len (int)

che number

rand $=n p$. random. $\operatorname{randint}(0$, len $($ int 1$))$

"f value of y data at position rand is smaller than current

set accordingly

if (int 1 [ rand $]<$ smallest 1$)$ :
smallest $1=$ int $1[$ rand $]$

smallest $=\operatorname{int} 1$ [rand]

\# equations if there are

\# more than 4 entries with
the same value at the beginning of the sample...

for $i$ in range $(0,100)$

newXData1 $=$
newXData2 $=$
$=11$

newYData1 $=$
newYData $=$
nos

highest number in $y$ dat

smallest $1=\max ($ int 1$)$
smallest $2=\max ($ int2)

slze as the original sample

for index in range $(0$, len (int 1$)$ :

index in range $(0$, len (int 1$)$ ):
\#choose a random number between 0 (inclusive) and the number

\# of elemnts cotas

rand $=n p$. random. $\operatorname{randint}(0, \quad$ len $($ int 1$))$

\# if value of $y$ data at position rand is smaller than current

\# smallest value

if $\quad($ int $1[$ rand $1<$ smallest 1$)$ :
smallest $1=$ int $1[$ rand $]$

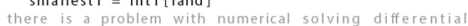

equations if there are

the same value at the beginning of the sample .

so of there is already 4 times the same smallest number in

next one would be the same

* choose a different one

(newrDatal.coun(smallest 1$)>=4$ and int 1

rand $]=$ smallest 1$):$
rand $=n p$.random . randint $(0$, len (int 1$))$

a corresponating values of $x$ and $y 1$ are added to the new lists

newXData 1 . append ( $f$ req $[$ rand $]$ )

* the subsamole is ordered by $x$ values (smallest to highest)

Imerical sovin

newXData1, newYData1 = zip (đsorted ( (zip (newXData1, newYData1))

for index in range $(0$, len (int 2$))$ :

(inclusive) and the number

of elemnts in

rand $=n p$. random. $\operatorname{randint}(0, \quad$ len $(\mathrm{int} 2))$

if value of $y$ data at position rand is smaller than current

smallest value, se:

if $($ int $2[$ rand $]<$ smallest 2$):$
smallest $2=$ int 2 [rand $]$

ats solving differential

Eequations if there are

so of there is already 4 times the same smallest number in

next one would be the same

while (newYData2. count ( smallest 2 ) $>=4$ and int2

rand $1=$ smallest 2 ):

corresponting datues of $x 2$ and $y 2$ are added to the new

newXData2. append ( $f$ re $q 2$ [rand])

the subsample is ordered by $x$ values (smallest to highest)
\# so of there is already 4 times the same smallest number in next one would be the same

while (newYData1. count(smallest 1) $>=4$ and int 1 (

rand $]=$ Smallest 1$):$
rand $=$ np. random . randint $(0$, len (int 1$))$
orresponding yase of $x$ and $y$ b are added to the new lists newXData 1 . append (freq [ rand $]$ )
newYData1. append ( int 1 [ rand $]$ )

to highest)

\# of diffe rential equations needs it that way
newXData1, newrData1 $=$ zip (đsorted (zip (newXData1, newYData1))

\# same for second data
for index in range $(0$, len $(i n t 2))$ :

en 0 (inclusive) and the number \# of elemnts in

rand $=n p \cdot$ random, $\operatorname{randint}(0, \quad$ len $(\operatorname{int} 2))$

if value of $y$
smallest value

if (int $2[$ rand $]<$ smallest 2 )

int 2 [ rand $]<$ mallest 2$)$
smallest $2=$ int2 $[$ rand $]$

there is a problem with numerical solving differential

equations if there are

so of there is already 4 times the same smallest number in

\# the sample but the
\# next one would be the same

choose a different one
while (newrData2. count(smallest2) $>=4$ and int 2[

rand $]=5$ mallest 2$):$
rand $=n p$.random. randint $(0$, len (int 2$))$

corde = spandesta) newXData2. append (freq2 [ rand] $]$ )
newYData2, append (int 2 rand $]$ )

newYData2. append (int 2 [rand])

\# because numerical soving

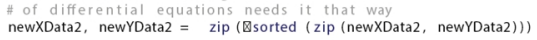

if $(i \% 5=0)$ :

end=' ', flush=True)

randomfit $=$ minimize $\left(o b j \_a u b, k_{-}\right.$opt_aub_diff

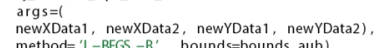

method=' 'L-BFGS
newDatafits_aub. append (randomfit. $x$ )

newData Fits_aub $=$ np. array (newDatafits_aub)
k_opt_aub $=$ np. mean(newDatafits_aub, 0 )

sigmalnterval $=1.0$

err_aub $=$ sigmalnterval $\mathbb{n p}$. std (newDatafits_aub, 0

print ('Inaub: InBootstrap \nMean:' , k_opt_aub, 'InError: ', err_aub, 'In')

print ('Standard error for system aub_kreis'

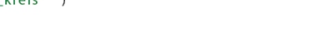

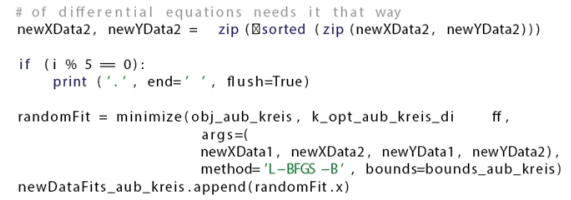

newDatafits_aub_kreis $=n p$. array (newDatafits_aub_krei $s)$
k_opt_aub_kreis $=$ np. mean (newDatafits_aub_kreis, 0 )

sigmalnterval $=1.0$

err_aub_kreis = sigmalnterval @ np.std (newDatafits_aub_kreis，0) print ('Inaub_kreis:InBootstrap InMean:' , k_opt_aub_kreis, 'InError: '
err_aub_kreis, 'In')

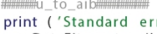

newDatafits_u_to aib = []

$i$ in range $(0,100)$

newXData1 $=11$
newXData2 $=11$

newYData1 $=$

ist of y data: initially set to be the

Fighest number in $y$ data

smallest1 $=\max ($ int 1$)$
smallest $2=\max ($ int2)

\# make subsamples the same size as the original sample

index in range (0, len (int 1)):
\#choose a random number between 0 (inclusive) and the number 4 ydatar (exclusive

rand $=n p$. random. randint $(0$, en (int 1$))$
\# if value of $y$ data at position rand is smaller than current

\# smallest value

if (int 1 [ rand $]<$ smallest 1 )
smallest $1=$ int 1 [rand]

there is a problem with numerical solving differential

\# the same value at the beginning of the sample...
" so of there is already 4 times the same smallest number in

the sample but the
thext one would be the same

\# choose a different one
while (newrData1.count(smallest1) $>=4$ and int1 [

rand $]=$ smallest 1$):$ :

correspontung yalues of $x$ and $y$ l are added to the new lists newXData 1 . append (freq $[$ rand $)$ )
newYData1. append (int 1 [ rand $]$ )

The subsample is ordered by $x$ values (smallest to highest)

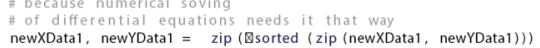

newXData1, newYDatal $=$ zip (囚sorted
\# same for second data
for index in range $(0$, len (int 2$))$ : 
\# choose a random number between 0 (inclusive) and the number Fof elemnts in
$*$ ydata2 (exclusive)

rand $=n$. random. randint $(0, \quad$ en (int2))
if value of $y$ data at position rand is smaller than current

if $($ int 2 [ rand $]<$ smallest 2$)$ :
smallest $2=$ int $2[$ rand $]$

* there is a problem with numerical solving differentia

more than 4 enert

the same value at the beginning of the sample...
so of there is already 4 times the same smallest number in

\# the sample but the

while (newYData2. count (smallest2) $>4$ and int2

rand $1=$ smallest 2$):$ :

(the new tists newXData2. append ( freq2 [ rand])
newYData2. append (int 2 [rand $]$ )

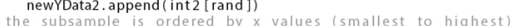

\# of differential equations needs it that way
newXData2, newYData2 $=$ zip (《sorted (zip (newXData2, newYData2))

if $(i \% 5=0)$ :

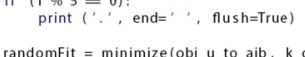

tabinize(obj_u_to_aib, k_opt_u_to_aib_diff

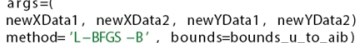

$\begin{aligned} & \text { method }==-B F G S-B \\ & \text { newDataFits_u_to_aib. append (random Fit. } x \text { ) }\end{aligned}$

newDataFits_u_to_aib $=n p$. array (newDatafits_u_to_aib)
k_opt_u_to_aib $=$ np. mean(newDatafits_u_to_aib, o)

sigmalnterval $=1.0$

err_u_to_aib = sigmalnterval $\mathbb{n p}$. std (newDatafits_u_to_aib, 0)

print ('Inu_to_aib:InBootstrapinMean:' , k_opt_u_to_aib, InError: ' ,

err_u_to_aib, 'In' )

print ('Standard error for system u_to_aib_kreis' ,

newDatarits_u_to_aib_kreis =

$i$ in range 10,
newXData $=[1]$

newYData $1=$

newYData1 $=$
newYData2 $=$

* highest number in $y$ dat

smallest2 $=\max ($ int 2

F make subsamples the same size as the original sampla

"choose a random number hetween 0 (inclusive) and the number \# of elemnts in

rand $=$ np.random. randint $(0$, len (int 1$)$

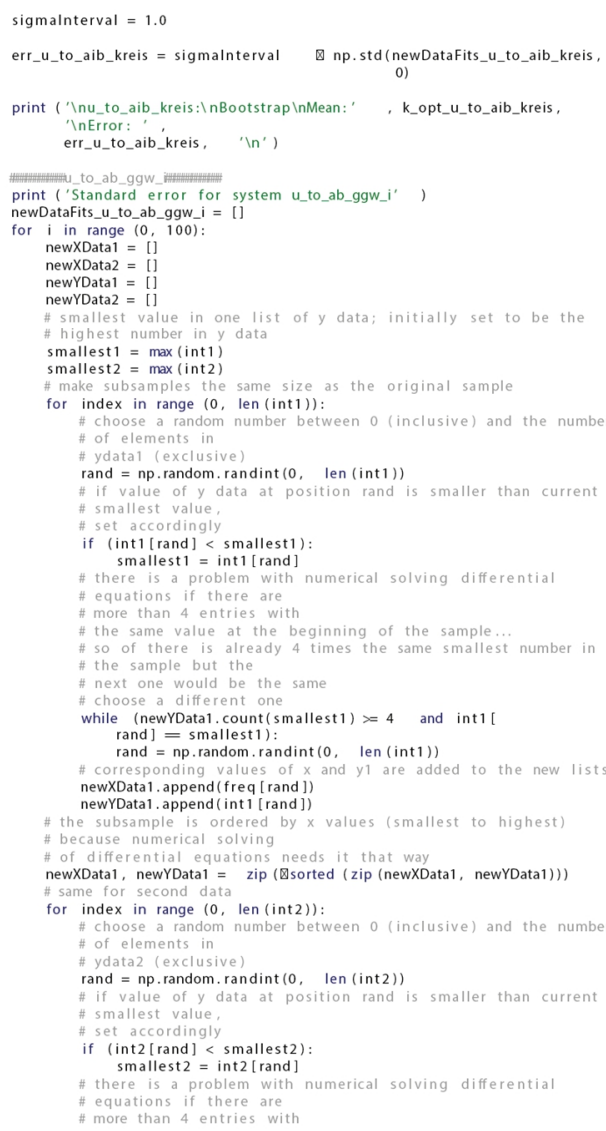

\# if value of $y$ data at position rand is smaller than curren

if $($ int 1 [rand $]<$ smallest 1$)$ :

smallest $1=$ int $1[$ rand $]$
\# there is a problem with

\# equations if there are

\# the same value at the beginning of the sample...
\# so of there is already 4 times the same smallest number in

\# next one would be the same

while (newYData1, count (smallest1) $>=4$ and int 1 [

rand $]=$ smallest 1$):$
rand $=$ np. random. randint $(0, \quad$ len $($ int 1$)\}$

corresponding values of $\mathrm{x}$ and $\mathrm{y} 1$ are added to the new lists newXData1. append (freq [ rand])
newYData1.append ( int1 [ rand])

* the subsample is ordered by $x$ values (smallest to highest)

newXData1, newYData1 $=$ zip (《sorted (Zip (newXData1, newYData1))

for index in range $(0$, len $($ int 2$))$ :

\# choose a random number between $O$ (inclusive) and the number

\# of elements in

rand $=$ np. random. randint $(0$, Ien (int 2$))$
\# if value of $y$ data at position rand is smaller than current

* smallest value, set

if (int $2[$ rand $]<$ smallest 2$)$ :

Smallest2 $=$ int $2[$ rand $]$
\# there is a problem with numerical solving differential.

\# equations if there are

\# the same value at the beginning of the sample...
\# so of there is already 4 times the same smallest number in

\# next one would be the same

while (newYData2. count (smallest 2 ) $>=4$ and int 2 I

rand $]=$ smallest 2$):$
rand $=$ np.random.randint $(0, \quad$ len $($ int 2$)$

ed to the new list newXata2.append (freq2 [rand])
newYData2. append (int2 [rand])

new ataz. append (int2 (rand])

because numerical soving

\# of differential equations needs it that way
newXData2, newYData2 $=z$ zip (đsorted ( $z$ ip (newXData2, newYData2))

if $(\mathrm{i} \% 5=0)$ :
print $\left({ }^{\prime},{ }^{\prime}\right.$, end $='$ ', flush=True $)$

randomfit $=$ minimize(obj_u_to_aib_kreis, $k_{\text {_opt_u_to_ai }}$ b_kreis_diff, args=(
newXata1, newXData2, newYData1, newYData2).
method= $=$ 'L-BFGS $-B^{\prime}$,

newDataFits_u_to_aib_kreis.append(randomito_t_at_kreis)

$\begin{array}{ll}\text { newDataFits_u_to_aib_kreis }=\text { np. array (newDatafits_u_t } & \text { o_aib_kreis) } \\ \text { k_opt_u_to_aib_kreis_ }=\text { np.mean(newDatafits_u_to_aib_k } & \text { reis, } 0 \text { ) }\end{array}$

\# the same value at the beginning of the sample...
\# so of there is already 4 times the same smallest number in \# the sample but the

while (newYData2. count (smallest 2 ) $>=4$ and int 2[
rand $]=$ smallest 2 : rand $]=$ smallest 2$):$
rand $=$ np.random .randint $(0, \quad$ len $($ int 2$))$

\# corresponding values of $x 2$ and $y 2$ are added to the new lists newXData2. append (freq2 ( rand $)$ )
newYData2. append ( int 2 (rand $])$

the subsample is ordered by $x$ values (smallest to highest) numerical solving

newXData2, newYData2 $=$ zip (đsorted ( $(z$ ip (newXData2, newYData 2$))$ ) if $(i \% 5=0):$
print $\left({ }^{\prime},{ }^{\prime}\right.$, end ${ }^{\prime}$ ', flush=True $)$

randomFit $=$ minimize (obj_u_to_ab_ggw_i, k_opt_u_to_ab $-\quad$ ggw_i_diff. args $=($ Cata1, newXData2, newYData1, newYData2),
newXDData method= ' $L-B F G S-B$ ', bounds=bounds_u_to_ab_ggw_i
newDataFits_u_to_ab_ggw_i. append (randomFit. $x$ ) newDatafits_u_to_ab_ggw_i $=$ np. array (newDatafits_u_to
k_opt_u_to_ab_ggw_i $i=$ np. mean (newDatafits_u_to_ab_ggw
ab_gow_i)
-i, 0 ) sigmalnterval $=1.0$

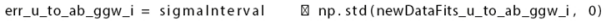
print (' 'Inu_to_ab_ggw_i: InBootstrap \nMean: ' , k_opt_u_to_ab_ggw_i.

err_u_to_ab_ggw_i, ' $\left(n{ }^{\prime}\right)$

print ('Standard error for system u_to_ab' ,

for $i$ in range $(0,100)$

newXData1 $=[]$

newXData2 $=$
newYData1 $=$

newrData1 $=4$
newYData $=[1$

smallest value in one list of $y$ data; initially set to be the

smallest $1=\max ($ int 1$)$

smallest $2=\max ($ int 2 )

make subsamples the same size as the original sample

index in range $(0$, len (int 1$)$ ):
$\#$ choose a random number between 0 (inclusive) and the number \# choose a random
$*$ of elements in
$*$

$\# y$ data 1 (exclusive)
rand $=$ np.random.randint $(0, \quad$ len (int 1$))$

\#if value of $y$ danta at positen (int) is smaller than current

* set accordingly

if $($ int 1 [rand $]<$ smallest1):
smallest $1=$ int $1[$ rand $]$

smallest $1=$ int 1 [rand $]$
*\# there is a problem with numerical solving differential

\# equations if there are
\# more than 4 entries with

\# the same value at the beginning of the sample...
\# so of there is already 4 times the same smallest number in
\# the sample but the 
\# next one would be the same

while (newYoata1. count(smallest1) $>4$ and int 1

rand $]=$ smallest 1$):$
rand $=n$ p. random. $\operatorname{randint}(0, \quad$ len $(i n t 1))$

\# corresponding values of $x$ and $y 7$ are added to the new lists

newXData 1 . append (freq [ rand])
newYDatal append ( int 1 [ rand $]$ )

the subsample is ordered by $x$ values (smallest to highest)

* because numerical solving

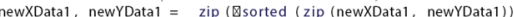

\# same for second data
for index in range $(0$, len (int 2$))$ :

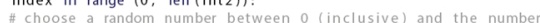

\# of elements in
\# ydataz exclusive

rand $=\mathrm{np}$. random. randint $(0, \quad$ len $(\mathrm{int} 2)$

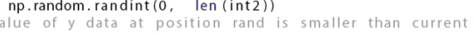

\# smallest value,

if (int2 [ rand $]<$ smallest 2$)$ :
smallest $2=$ int 2 [ rand $]$

smallest $2=$ int $2[$ rand $]$
there is a problem with numerical solving differential

\# there is a problem with

\# more than 4 entries wit

政

smallest number in

\# the sample but the
\# next one would be the same

while (newrData2. count (smallest2) $>=4$ and int 2 |

rand $]=$ smallest 2 ):

rand $=n p$. random. randint $(0$, len (int2))
corresponding values of $x 2$ and $y 2$ are added to the new 11 sts newXData2. append (freq 2 ( rand

the subsample is ordered by $x$ values (smallest to highest)

* because numerical soving

newXData2, newYData2 $=$ zip (Bsorted ( zip (newXData2, newYData2))

if $(i \% 5=0)$

print $(\cdots$, : end=' ', flush=True

randomfit = minimize (obi_u_to_ab, k_opt_u_to_ab_dift, args (
newData1, newXData2, newYData1, newYData2),
method= $L-B F G 5-B^{\prime}$, bounds=bounds_u_to_ab)

$\log ^{2}$

newDatarits_u_to_ab $=$ np. array (newDatafits_u_to_ab)

sigmalnterval $=1.0$

err_u_to_ab = sigmalnterval $\mathbb{Q n p}$. std (newDatafits_u_to_ab, 0)

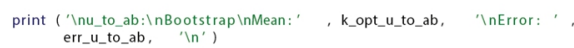

print the optimized results by differential evolution for each systen print ("Final Values k_opt_aub:, K_opt_aub_diff)
print (" Final Values k_opt_aub_kreis: ", k_opt_aub_kreis_diff)

'it itu_to_ab_ggw_ilt

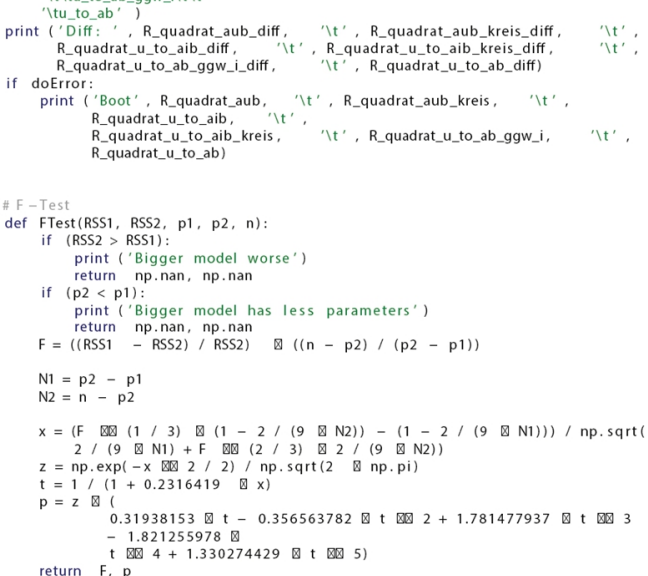

print ('F-Value. auth aub_kreis

F_aub_aub_kreis, P_aub_aub_kreis = FTest(RSS_aub_diff, RSS_aub_kreis_diff,

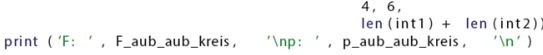

F_aub_u_to_aib, P_aub_u_to_aib $=$ FTest(RSS_aub_diff, RS SS_u_to_aib_diff, 4, print ('F: ', F_aub_u_to_aib, 'Inp: ', P_aub_u_to_aib, $\begin{gathered}\text { len (int1) } \\ \text { (Int') }\end{gathered}$

F_aub_u_to_aib_kreis, P_aub_u_to_aib_kreis = FTest(RSS $\begin{gathered}\text { aub_diff, } \\ \text { RSS_u_to_aib_kreis_diff, }\end{gathered}$

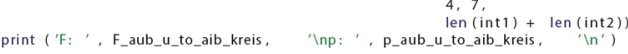

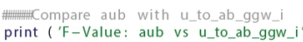

F_aub_u_to_ab_ggw_i, P_aub_u_to_ab_ggw_i = FTest(RSS_a $\begin{gathered}\text { ub_diff, } \\ \text { RSS_u_to_ab_gm_iddiff, 4, }\end{gathered}$

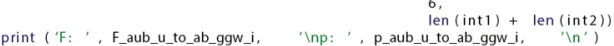

Numpare aub with u to aib
print ('F-Value: aub vs u_to aib

print ('F-Value: aub u_to aib kreis

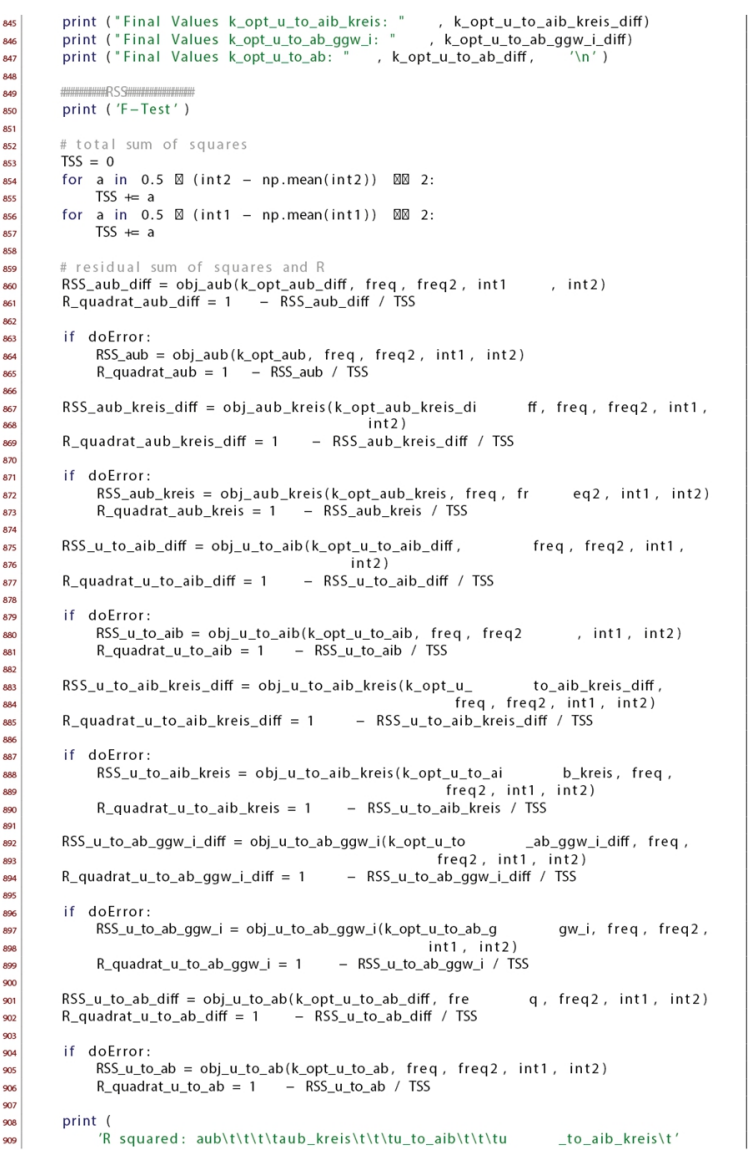

\footnotetext{
Mencompare aub with u_toab

print ('F-Value: aub vs u-to_ab' ')
F_aub_u_to_ab, P_aub_u_to_ab = FTest(RSS_aub_diff, RSS
u_to_ab_diff, 4, 6 , print ('F: ', F_aub_u_to_ab, Inp: ', P_aub_u_to_ab, $\left(\ln n^{\prime}\right)$

\#rint ( F-Value uto ab with aub kreis

F_u_to_ab_aub_kreis, p_u_to_ab_aub_kreis = FTest(RSS_u $\begin{gathered}\text { to_ab_diff, } \\ \text { RSS_aub_kreis_diff, 4, }\end{gathered}$

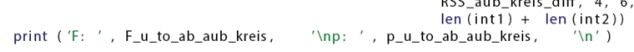

print ('F-V U_to_ab with u_to_aib

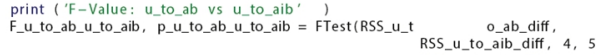

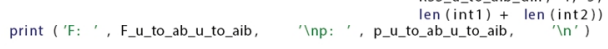

Compare u_to_ab with u_to_aib_kreis
print ('F-Value: U_to_ab vs u_to_aib_kreis'

Print
F_u_to_ab_u_to_aib_kreis, P_u_to_ab_u_to_aib_kreis $=F \quad$ Test (RSS_u_to_ab_diff, RSS_u_to_aib_kreis_diff,

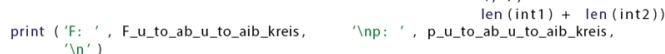

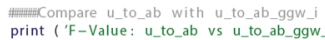

print ('F-Value: u_to_ab vs u_to_ab_ggw_i' ' '
F_u_to_ab_u_to_ab_ggw_i, p_u_to_ab_u_to_ab_ggw_i = FTe

St (RSS_u_to_ab_diff,
RSS_L_to_ab_agw_idiff.

4,6
len $($ int 1$)+(e n($ int 2$))$

print ('F: , , F_u_to_ab_u_to_ab_ggw_i, Inp: ', p_u_to_ab_u_to_ab_ggw_i,

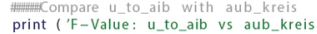

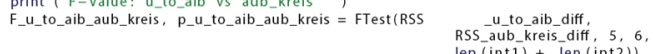

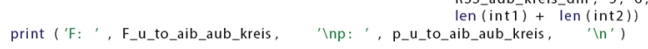

\#-Compare U_to_aib with U_to_aib_kreis

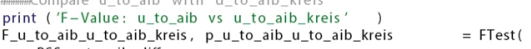

RSS_u_to_aib_diff,
RSS_uto_to _aib_kreis_diff, 5,7 ,

print (' $\left(\right.$ : $\left(n^{\prime}\right)$ ) , F_u_to_aib_u_to_aib_kreis, 'Inp: ', , p_u_to_aib_u_to_aib_kreis,

print ('F-Value: u_to_aib vs u_to_ab_ggw_i'
F_u_to_aib_u_to_ab_ggw_i, p_u_to_aib_u_to_ab_ggw_i $=$ F $\quad$ Test

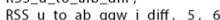

len (int1) + len (int 2 )

len (int1) + len (int 2$)$ )
print ('F, , , F_u_to_aib_u_to_ab_ggw_i, Inp: ', , p_u_to_aib_u_to_ab_ggw_i,
} 
print ('F-Value: aub_kreis vs u_to_aib_kreis'
F_aub_kreis_u_to_aib_kreis, p_aub_kreis_u_to_aib_kre $\quad$ is = FTestl

RSS_aub_kreis_diff,
RSS_u_to_aib_kreis_diff, 6,7
len (int1)+ len (int2))

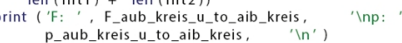

Hempare u_to_ab_ggw_i with u_to_aib_kreis

print ('F-Value: U_to_ab_ggw_i vs u_to_aib_kreis'
E_u_to_ab_gaw_i_u_to_aib_kreis, p_u_to_ab_agw_i_u_to

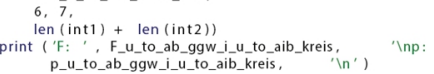

print ('FITING PARAME
if doError:

print ('aub:InParameters: ', k_opt_aub_diff, 'InError: ', err_aub, ' $(n ')$

print ('er__to_alabrinparameters: ', , k_opt_u_to_aib_diff, InError:

print ('u_to_aib_kreis: $\backslash$ nParameters: , , k_opt_u_to_aib_kreis_diff

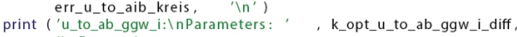

print ('u_to_to_ab:InParameters: ', ) , k_opt_u_to_ab_diff, InError: '

else

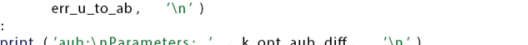

print ('aub: InParameters: ', K_opt_aub_diff, 'In')
print ('aub_kreis:InParameters:, ', k_opt_aub_kreis_diff, (In')

print ('u_to_aib_kreis: InParameters: , , , k kopt_opt_u_to_aib_kreis_diff,

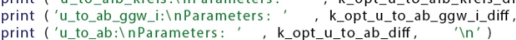

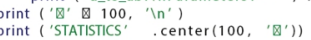

print

'R squared: |t|t|taub|t|t|taub_kreis|t|t|tu_to_aib|t|

"Itu_to_aib_kreis It tt ttu_to_ab_ggw__t

print ('Globally Optimized: ', R_quadrat_aub_diff. At'

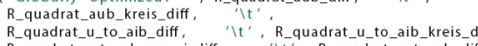

print ('F-Test: ')
print ('aub vs aub_kreis: ', ' 'F: ', F_aub_aub_kreis, Itp: '

P_aub_aub_kreis)
print ('aub vs u-_to_aib: ', 'F: ', F_aub_u_to_aib, Itp:
print ('aub vs u_to_aib_kreis: ', P_aub_u_to_aib

P_aub_u_to_aib_kreis)

P_aub_u_to_aib_kreis)
(aub vs u_to_ab_gg_i:
p_aub_u_to_ab_ggw_i)

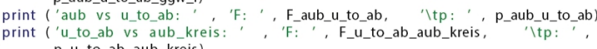

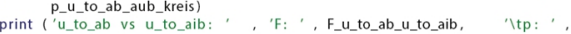
RSS_u_to_ab_ggwi-difif,
RSS_u_to_aib_kreis_diff,

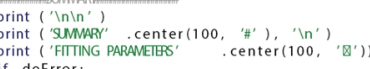

print ('aub_kreis:InParameters: ', ', k_opt_aub_kreis_diff, 'InError: '
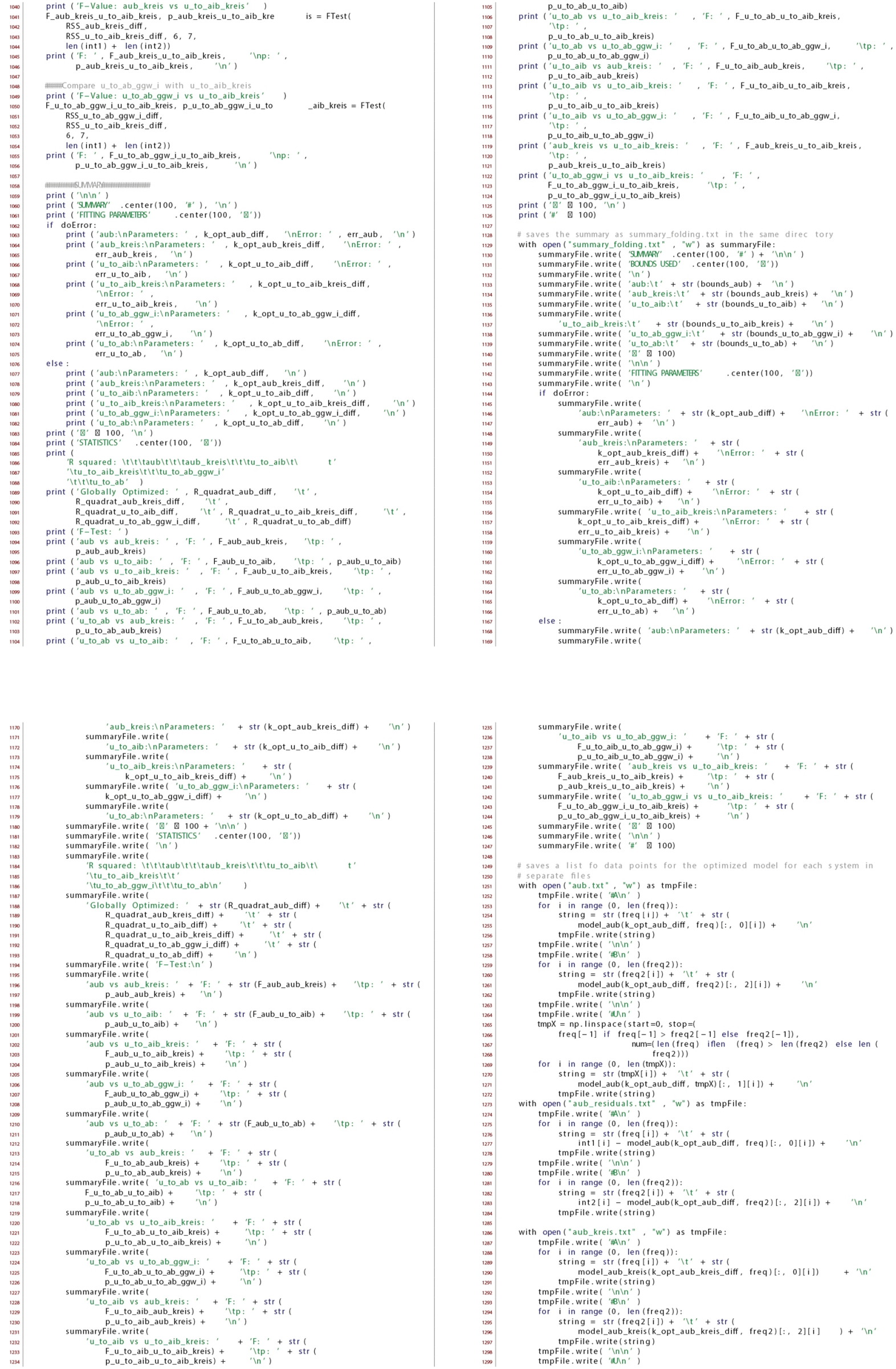

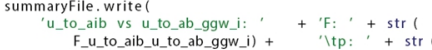

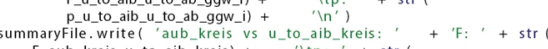
F_aub_kreis_u_to_aib_kreis) + 'Itp: ' + str (

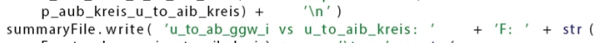

$+\operatorname{str} 1^{+}$ p_u_to_ab_ggw_i_u_to_aib_kreis

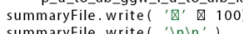

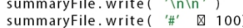

* saves a list fo data points for the optimized model for each system in

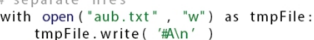

for $i$ in range $(0$, len (freq)):
string $=$ str (freq $(i j)+$

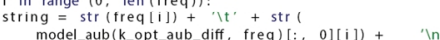

tmpFile. Write (string
ImpFile. write (' $I n ! n ')$

tmpFile. write( 'Bßin'

i in range $(0$, len (freq2)):
string $=\operatorname{str}($ freq $2[i])+$

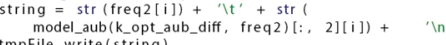

tmpFile. Write (string
tmpFile. write ( Inln')
tmpFile, write( 'Unn',

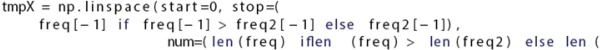

for $\begin{aligned} & i \text { in range }(0, \text { len (tmpX)) } \\ & \text { string }=\operatorname{str}(\operatorname{tmp} X[i])+\end{aligned}$

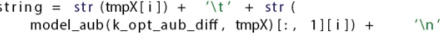

tmpFile. Write(string)
with open ("aub_residuals,txt: " "w") as tmpFile

tmpFile. write( 'Alln' ')
for $i$ in range $(0$, , len (freq))

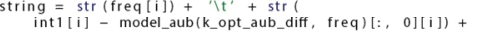

tmpFile. write(string)

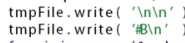

for $i$ in range $(0$, len (freq 2$))$ :

string $=$ str (freq2[i]]+ + it' + str (
int2 $[i]-$ model_aub(k_opt_aub_diff, freq2 $)[:, 2][i])+$

tmpFile. write(string)

with open ("aub_kreis.txt," , "W") as tmpFile
tmpFile.write( "MAnn"

tmpFile. Write ( 'Aln' $)$
for $i$ in range $(0$, len (freq))

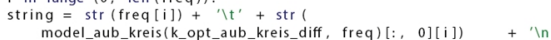

model_aub_kreis (k_o
tmpFile.write(string)

tmpFile. write ( 'Inln')

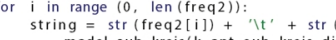

model_aub_kreis(k_opt_aub_kreis_diff, freq2) [:, 2][i] , ) + tmpFile. writer ('Inn')
tmpFile. write( 'Aln' ) 
$\operatorname{tmpX}=$ np. Iinspace $($ start $=0$, stop $=($
freq $[-1]$ if freq $[-1]>$ freq2 $[-1]$ else freq $2[-1])$

freq2))

in range $(0, \operatorname{len}(\operatorname{tmp} X))$ :

shingente

tmpFile.write(string)
open ("aub_kreis_residuals.txt", " $w^{*}$ ) as tmpFile:

for $i$ in rite

sing $=\operatorname{str}($ freq[i]) + it $+\operatorname{str}($

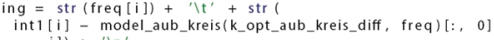

tmpFile. write(string)

tmpFile. write( 'Inin'

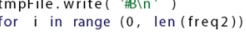

sing $=\operatorname{str}($ freaz $[i])+t^{\prime}+\operatorname{str}$

int2[i] - model_aub_kreis(k_opt_aub_kreis_diff, freq2) [:, 2]!

i] $)+$ ' $I$ '
tmpFile. write (string)

with open ("u to aib.txt" , "w") as tmpFile

tmpFile. writel 'AAln' $j$ (freq))
for $i$ in range $(0$, len (fres

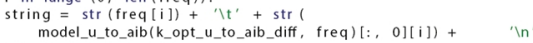

model_u_to_aib(k_ot
tmpFile,write (string)

tmpFile. write( ' $I n \backslash n$ ')
tmpFile. write( ' $B \backslash n^{\prime}$ ')

for $i$ in range $(0$, len (freq2)):

string $=\operatorname{str}($ freq2 $[i])+$ it' + str
model_u_to_aib(k_opt_u_to_aib_diff, freq2) [:, 2][i]) + in

tmpFile.write(string)

tmpFile. write( ' ' $n$ in'
tmpFile. write( $\# I n$ '

$=n p$. linspace (start $=0$, stop $=($
freq $[-1]$ if freq $[-1]>$ freq $2[-1]$ else freq $2[-1])$

for $i$ in range $(0$, len $(\operatorname{tmp} x))$ :

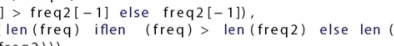

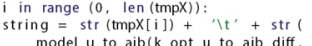

model_u_to_aib(K_opt_u_to_aib_diff, tmpX) [:, 1][i]) + ${ }^{\prime} n^{\prime}$

mpFile.write( 'In!n')

tmpFile. writer 'fuln

$i$ in range $(0, \operatorname{len}(\operatorname{tmp} \mathrm{X}))$ :

string $=\operatorname{str}\left(\right.$ tmpXX[i]) + $+t^{\prime}+$ str (
model_u_to_aib(k_opt_u_to_aib_diff, tmpX) [:, 3$][i])+$

tmpFile.writes (string

政") as tmpFile:

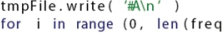

in range $(0$, len (freq $)):$
sting $=\operatorname{str}($ freq $[i])+t^{\prime}+\operatorname{str}$

int 1 [i] - model_u_to_aib(k_opt_u_to_aib_diff, freq) [:, 0]

tmpFile. write(string)

tmpFile. write( 'Inln'
tmpFile.write( ' $B$ Bin'

for $i$ in range $(0$, len (freq 2$))$.

string = str (freq2[i])+ $4 t^{\prime}+\operatorname{str}$

i] $)+$ ' $I n '$
tmpFile. write(string)

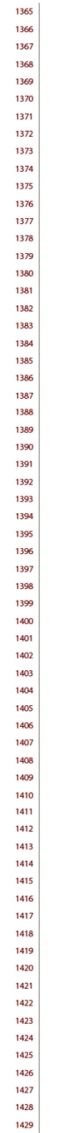

with open ("u_to_aib_kreis,txt", " $w$ ") as tmpFile:
tmpFile.write( "HAln',

for $i$ in range $(0$, len (freq))

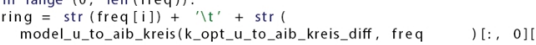

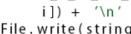

tmpFile.write ' ' $\ln \left(n n^{\prime}\right.$ ')

tmpFile. writel 'ABin'
for it in range (

$i$ in range $(0$, len (freq 2$))$ :

string $=$ str (freq2[i]) + It' + str
model_u_to_aib_kreis(k_opt_u_to_aib_kreis_diff, freq

tmpFile, write(string)

tmpFile. Writer 'inn'
tmpFile. writel $\# \backslash n$ '

mpX $=n p$. linspace(start $=0$, stop $=($
freq $[-1]$ if freq $[-1]>$ freq2 $[-1]$ else freq $2[-1])$,
num=(len (freq) iflen (freq) $>$ len (freq2) else len (

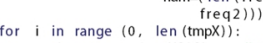

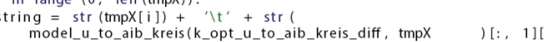

i $))+$ ' 'In'
tmpFile. write(string)

tmpFile. write( 'In'n'

for $i$ in range $(0$, en $($ tmp $x)$ )

in range $(0, \operatorname{len}(\operatorname{tmp} X)):$
string $=\operatorname{str}(\operatorname{tmp} X[i])+t^{\prime}+\operatorname{trt}$

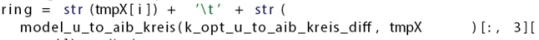

tmpFile,write(string)

with open ("U_to_aib_kreis_residuals.txt" , "w") as tmpFile:

tmpFile. Writes 'Aln' '
for i in range $(0$, en (freq)):

$i$ in range $(0$, len (freq)):
string $=$ str (freq $(i))+\left(t^{\prime}+\operatorname{str}(\right.$

string $=$ str
int1 $1[i]-$

model_u_to_aib_kreis(k_opt_u_to_aib_kreis_diff, freq ) [:, 0][

tmpFile.write(string)

tmpFile. Write ( IInn')
tmpFile. write( 'fBin' )

tmpFile. Write( 'ABin' '
for $i$ in range $(0$, len (freq 2$)$ ).

string = str (freq2[i]) + "tt' + stri

int2 [i] -
model_u_to_aib_kreis(k_opt_u_to_aib_kreis_diff, freq

tmpFile, write(string)

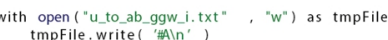

tmpFile. Writel "Ain' '
for $i$ in range $(0$, Ien (freq)):

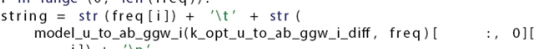

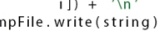

tmpFile. write (strin
tmpFile. writer ' In!n')
tmpFile writel 'B

tmpFile. writei
tmpFile. write
for $i$ in $r a n g$

or $i$ in range $(0$, len (freq 2$)$ )

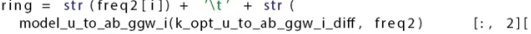

tmpFile. write (string
tmpFile. write( ' $(n \ln$ ')

tmpFile. Write( 'tflın'
tmpX $=$ np. linspacelst

$=n p$, linspace (start $=0$, stop $=($
freq $[-1]$ if freq $[-1]>$ freq $2[-1]$ else freq $2[-1])$,
num $=($ len (freq) iflen (freq) $>$ len (freq 2$)$ else len

for $i$ in range $(0$, en $($ freq 2 , $($ req2 2$)))$
(mpX) :

i]) + (n!
inpFile. write (string)

mpFile. writel 'In'n'

tmpFile, write ('tuln' $)$
for $i$ in range $(0$, Ien (tmpX))

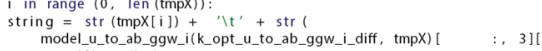

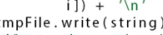

tmprile. Write( string)
open ("u_to_ab_ggw_i_residuals.txt" , "w") as tmpFile:

tmpFile. Write ( 'AAin'
for $i$ in range $(0$. Ien (freq)):

i in range (0, len (freq)):

int 1 [i] -
model_u_toab_ggw_i(k_opt_u_to_ab_ggw_i_diff, freq) $[\quad \therefore, 0] l$

tmpFile, write(string)

tmpFile. write ( IInn')

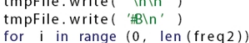

$i$ in range $(0$, len (freq2 $)):$
string $=\operatorname{str}($ freq2 $[i])+4 t^{\prime}+\operatorname{str}$

$\operatorname{string}=\operatorname{str}($ freq2$[i])+' \mid t t^{\prime}+\operatorname{str}($
int $2[i]-$

model_u_to_ab_ggw_i(k_opt_u_to_ab_ggw_i_diff, freq2 ) [:, 2]

tmpFile, write (string)

with open ("u_to_ab. txt", " "w") as tmpFile:
tmpFile.write( "AAln',

tmpFile. Write ( 'Alin', ')
for i in range ( 0 , len (freq)):

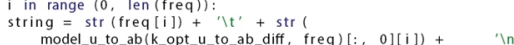

tmpFile. writelstring

ImpFile. writel 'Inln'

mpFile. Write( 'Blin'
for $i$ in range $(0$, len (freq2))

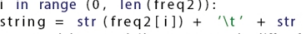

model_u_to_ab(k_opt_u_to_ab_diff, freq2) [:, 1][i]]) + in

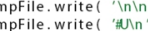

$X=n p$. linspace (start $=0$, stop

freq $[-1]$ if freq $[-1]>$ freq2 $[-1]$ else freq $2[-1])$,
num=(len (freq) iflen (freq) $>$ len (freq 2$)$ else len freq 2$)))$
$(\operatorname{tmp} x)):$

for $i$ in range $(0, \operatorname{len}(\operatorname{tmp} X))$ :

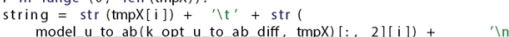

tmpFile. write(string)

with open ("U_to_ab_residuals.txt" , "w") as tmpFil

tmpFile. Write( 'Aln' $)$ '
for i in range $(0$, len (freq)):

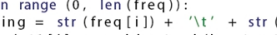

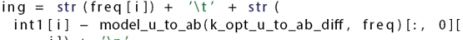

tmpFile. write(string

tmpFile. write(string)
tmpFile. write( 'Inn')
tmpFile. write( 'ABin'")

for $i$ in range $(0$, len $($ freq 2$)):$
string $=$ str (freq2 $[i])+$ it + str (
int2 $[i]-$ model_u_to_ab (k_opt_u_to_ab_diff, freq2 $)[:, 2]$ i]) + ' $I n$ '
tmpFile. write (string)

subplot (231)
title ('aub')

plot(freq2, int 2, ' $k$ ')

plot(freq, int1, 'k', )
plot(freq, model_aub(k_opt_aub_diff, freq) $[:, 0], \quad$ ' $r$ ', linewidth $=3.0)$

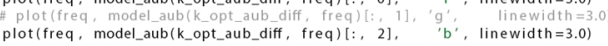

subplot (232)

title( 'aub_kreis')
plot(freq2, int 2, ,

plot(freq, model_aub_kreis(k_opt_aub_kreis_ditt, freq $1:$, , 01, ' $r$ ',

linewidth=3.0)
plot(freq, model_aub_kreis(k_opt_aub_kreis_diff, fre 9 ) $[:, 11$, ' 9

plot(freq, model_aub_kreis(k_opt_aub_kreis_diff, freq $)[:, 2], b^{\prime}$,

subplot (233)

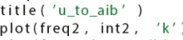

plot(freq2, int2, ' $k$ ')

plot(freq, model_u_to_aib(k_opt_u_to_aib_diff, freq) [ $\quad \therefore, 0],{ }^{\prime} r$ ',

plot(frea, model_u_to_aib(k_opt_u_to_aib_diff, freq) [ $\quad:, 1], \quad \mathrm{g}$ '

plot(freq, model_u_to_aib(k_opt_u_to_aib_diff, freq) [ $\quad, 2]$, 'b'

linewidth $=3.0)$
plot(freq, model_u_to_aib(k_opt_u_to_aib_diff, freq) [:, 3], yellow

subplot (234)
title(')

(the ( u_to_aib_kreis'

plot(freg, int1, ' $k$ '.

plot (freq, model_u_to_aib_kreis(k_opt_u_to_aib_kreis

linewidth $=3.0)$
pot(freq. model $u-10$

, nodel_u_to_aib_kreis(k_opt_u_to_aib_kreis

plot (freq, model_u_to_aib_kreis (k_opt_u_to_aib_kreis

s.diff, freq) [:, 3]

linewidth=3.0)

subplot (235)

plot(freq2, int2,

' $k$ '

ot (freq, model_u_to_ab_ggw_i(k_opt_u_to_ab_ggw_i_d iff, freq) [:, 0], ' $r$ '

plot (freq, model_u_to_ab_ggw_i(k_opt_u_to_ab_ggw_i_d iff, freq) [:, 1], ' $g$ '

pinewidth $=3.0)$
plot (freq, mode_u $t$ to_ab_ggw_i(k_opt_u_to_ab_ggw_i_d

plot (freq, model_u_to_ab_ggw_i (k_opt_u_to_ab_ggw_i_ diff, freq) [:, 3],
cyellow', linewidth $=3,0)$ 
subplot (236)

plot(freq2, int 2, , $k$ ')

plot (freq, int1, ' ' $k$ ', ')
ploteq, model_to_ab(k_opt_u_to_ab_diff, freq) [:, o], ' $r$ '

linewidth $=3.0)$
plot(freq, mode__u_to_ab(k_opt_u_to_ab_diff, freq) [:, 1], ' $\mathrm{b}$ ',
linewidth $=3.0$ )

plot(freq, model_u_to_ab(k_opt_u_to_ab_diff, freq) [: , 2], 'g

savefig ( 'folding_all, pdf', format ='pdf', transparent=True

(If ()

title( 'aub')
plot (freq2, in

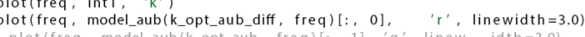

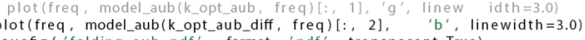
savefig ('folding_aub. pdf', format ='pdf', transparent=True)

If ()

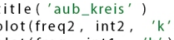

plot(freq, model_aub_kreis(k_opt_aub_kreis_diff, freq ) $[:, 0]$, ' $r$ '

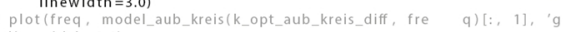

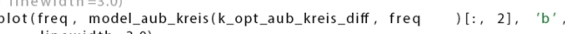

linewidth $=3.0)$
savefig( ('folding_aub_kreis. pdf', , format ='pdf', transparent=True)

cIf()

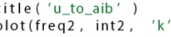

plot(freq, model_u_to_aib(k_opt_u_to_aib_diff, freq) [ $\quad:, 0], \mathrm{r}^{\prime}$

linewidth=3.0)
plot(freq, model_u_to_aib(k_opt_u_to_aib_diff, freq) [ $\quad: 11, g^{\prime}$

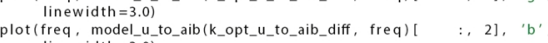

plot_ffeq, model_u_to_aib(k_opt_u_to_aib_diff, freq) [:, 3], 'yellow

savefig ('folding_u_to_aib.pdf' , format ='pdf', transparent=True)

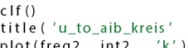

plot (freq2, int 2 , ' ' $k$ ')

plot (freq, model_u_to_aib_kreis(k_opt_u_to_aib_kreis

plot (freq, model_u_to_aib_kreis(k_opt_u_to_aib_kreis

ot (freq, model

to_aib_kreis(k_opt_u_to_aib_kreis

diff, freq) $[:, 1], \quad g$

( cIf (I)

clf()

title ( 'Residuals u_to_ab_ggw_i' ')
plot(freq, int1 - model_u_to_ab_ggw_i(k_opt_u_to_ab_ggw_i_diff, freq) [ : , 0], markerfacecolor $=(1,0,0,0.5)$,

plot (freq2,
int2 int2 2 - model_u_to_ab_ggw_i(k_opt_u_to_ab_ggw_i_diff, freq2 $) \quad[:, 2], \quad$, markerfacecolor $=(0,0,1,0.5)$

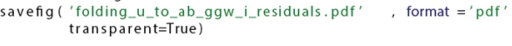

clf ()
title(

itlel ('Residuals u-to ab' )

(freq, int 1 - modelu_to_ab(k_opt_u_to_ab_diff, freq) $[:, 0], \quad \mathrm{o}^{\prime}$ ',
markerfacecolor=(1, 0, 0, 0.5),

plot (freq2, int2 - model_u_to_ab(k_opt_u_to_ab_diff, freq2) $[:, 1], \quad{ }^{\prime}$,
markerfacecolor $=(0,0,1,0.5)$,

markeredgecolor=' $k$ ')
savefig ('folding_u_to_ab_residuals. pdf' , format ='pdf', transparent=Irue)

(If ()

If ()
subplot (231)

title('Residuals aub'

(freq, int - model_aub(k_opt_aub_diff, freq) [:, 0], 'o'

markeredgecolor $=\left(\mathrm{k}^{\prime}\right.$, , markersi

ot (freq2, int2 - model_aub(k_opt_aub_diff, freq2) $[:, 2], \quad, 0^{\prime}$,

markeredgecolor $={ }^{\prime} k$, markersize $=3$, markeredgewidth $=0.5$ )

subplot $(232)$

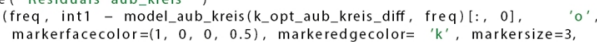

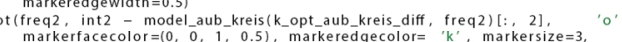
markerfacecolor $=(0,0$,
markeredgewidth $=0.5)$

subplot (233)

subplot(233)
title ('Residuals u_to_aib')
plot(freq, int $1-$ model_u_to_aib(k_opt_u_to_aib_diff, freq) $[:, 0]$,
markerfacecolor $=(1,0,0,0.5)$, markeredgecolor $={ }^{\prime}, k^{\prime}$, markersize $=3$,

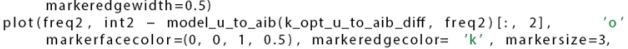

markerfacecolor $=(0$,
markeredgewidth $=0.5$

subplot (234)

title ('Residuals u_to_aib_kreis'

plot(frea,

int 1 ' - model_u_to_aib_kreis(k_opt_u_to_aib_kreis_diff, freq

markerfacecolor $=(1,0,0,0.5)$

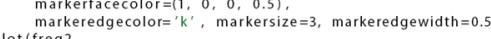
int2, modet_nto to

markerfacecolor $=\left(\begin{array}{lll}0,0 & 0,1,0.5)\end{array}\right.$

$[\cdot, 0]$

2) [:, 2] title ( 'u_to_ab_ggw_i'
plot (freq2, int2, ${ }^{\prime}, k^{\prime}$ )

plot (freq, model_u_to_ab_ggw_i(k_opt_u_to_ab_ggw_i_d iff, freq) [:, 0], ' $r$ ',

linewidth =3.0)

plot (freq, model_u_to_ab_ggw_i(k_opt_u_to_ab_ggw_i_d iff, freq) [:, 1], ' $g$ ',

plot (freq, model u_to_ab_ggw_i(k_opt_u_to_ab_ggw_i_d iff, freq) [:, 2], 'b'

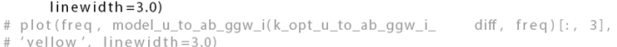

savefig ('folding_u_to_ab_ggw_i. pdf' , format ='pdf', transparent=True)

cIf ()

title( 'u_to_ab'
plot (freq2, int2,

plot (freq, int 1, ' $k$ '

plot (freq, model_u_to_ab(k_opt_u_to_ab_diff, freq) [:, 0$],$, ' $r$ ',
linewidth $=3,0)$.

plot (freq, model_u_to_ab(k_opt_u_to_ab_diff, freq) [:, 1], 'b',

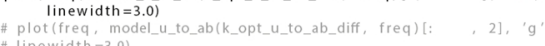

savefig ('folding_u_to_ab.pdf' , format ='pdf', transparent=True)

clf ()
title('Residuals aub')

lot (freq, int1 - model_aub(k_opt_aub_diff, freq) [:, 0],

markerfacecolor $=(1,0,0,0.5)$
markeredgecolor $\left.='{ }^{\prime},{ }^{\prime}\right)$

plot (freq2, int2 - model_aub(k_opt_aub_diff, freq2) [:, 21, ' $0^{\prime}$

markerfacecolor $=(0$,

savefig ('folding_aub_residuals, pdf' , format =' $p d f$ ', transparent=True)

cIf ()

title( 'Residuals aub kreis'

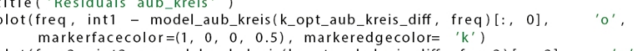
plot freq 2 int 2 model aub markerfacecolor $=(0,0,1,0.5)$, markeredgecolor= ' $k$ ' )
savefig ('folding_aub_kreis_residuals. pdf', format ='pdf', transparent=True)

cIf ()

(1)

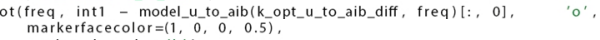

plot(freq2, int2 - model_u_to_aib(k_opt_u_to_aib diff, freq2) $\left[\begin{array}{ll}: & 2\end{array}\right], \mathrm{o}^{\prime}$. markerfacecolor $=(0,0,1,0.5)$

markeredgecolor=' $k^{\prime}$ ')
savefig( 'folding_u_to_aib_residuals. pdf' , format ='pdf', transparent=True)

Clf ()
title('Residuals u_to_aib_kreis' ,

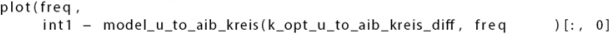

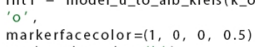

markeredgecolor $={ }^{\prime} k$ ')

(ite

markerfacecolor $=(0,0,1,0.5)$
markeredgecolor $=' k$ ' $)$

markeredgecolor $={ }^{\prime} k$ ' markersize $=3$, markeredgewidth $=0.5$ )

subplot (235)

title( Residuals u_to_ab_ggw_i'
plot(freq, int1 - model_u_to_ab_ggw_i(k_opt_u_to_ab_ggw_i_diff, freq) $[\quad:, 0]$

markerfacecolor $=\left(\begin{array}{lll}0_{1} & 0,0, & 0.5\end{array}\right)$

mareracecolor $(1,0,0,0.5)$,

plot (freq2,

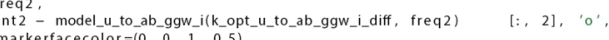

markerfacecolocolor $=(0,0,1,0.5), \quad, \quad$ markersize $=3$, markeredgewidth $=0.5)$

subplot (236)
title('Residuals u_to_ab' ,

plot(freq, int1 - model_u_to_ab(k_opt_u_to_ab_diff, freq) [:, 0], 'o markeredgecolor $=$ ' $k$ ' ' markersize $=3$, markeredgewidth $=0,5)$

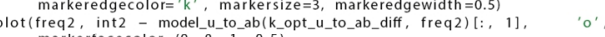

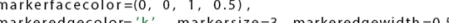

savefig ('folding_all_residuals, pdf' , format ='pdf', transparent=True) 
Acknowledgements - Danksagung

"... und vergiss nicht, Danke zu sagen!"

Meine liebe Mama, immer

Ich danke meinem Doktorvater und Mentor Harald Schwalbe;
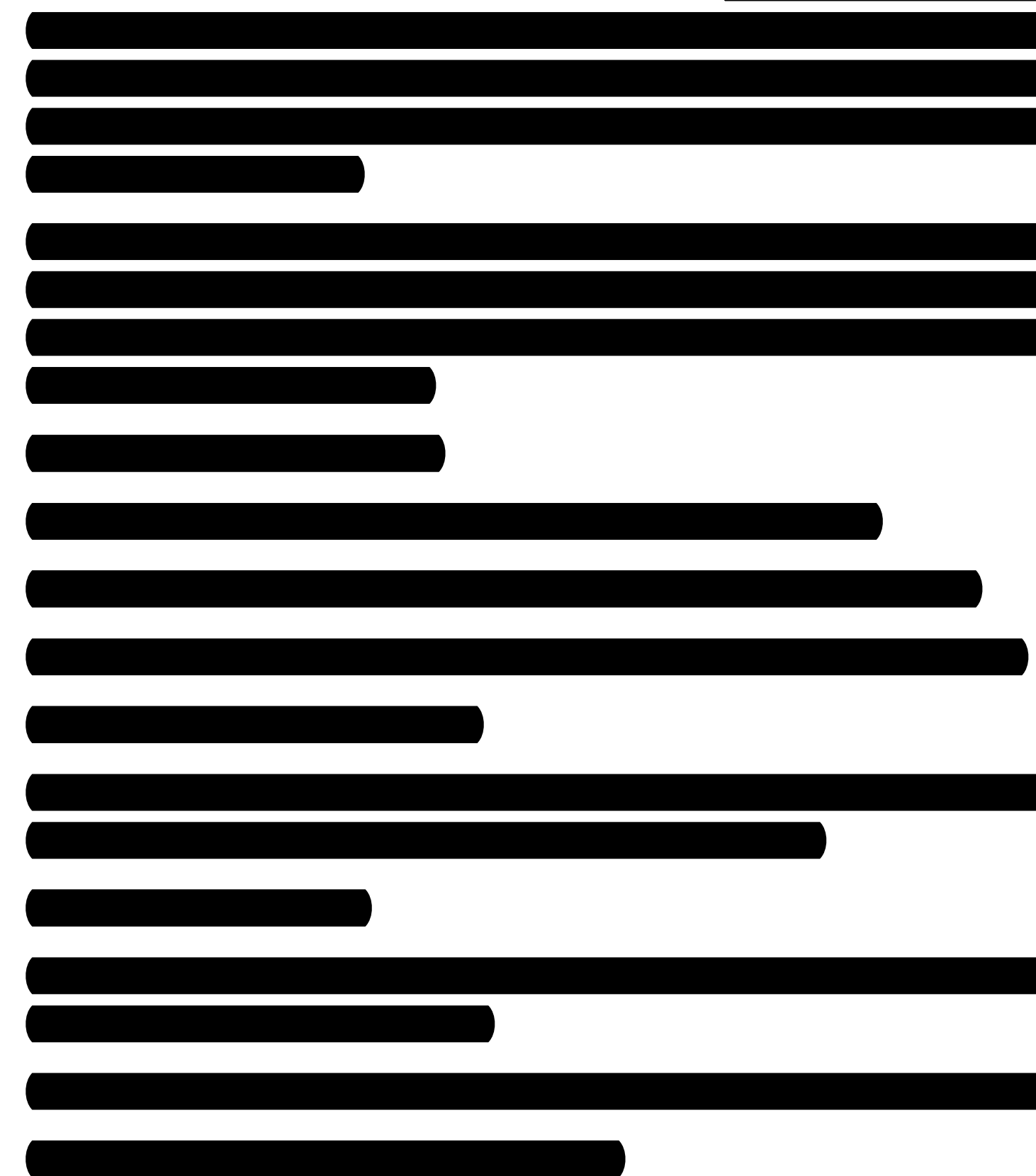

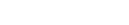

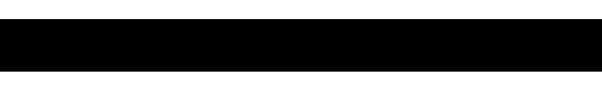




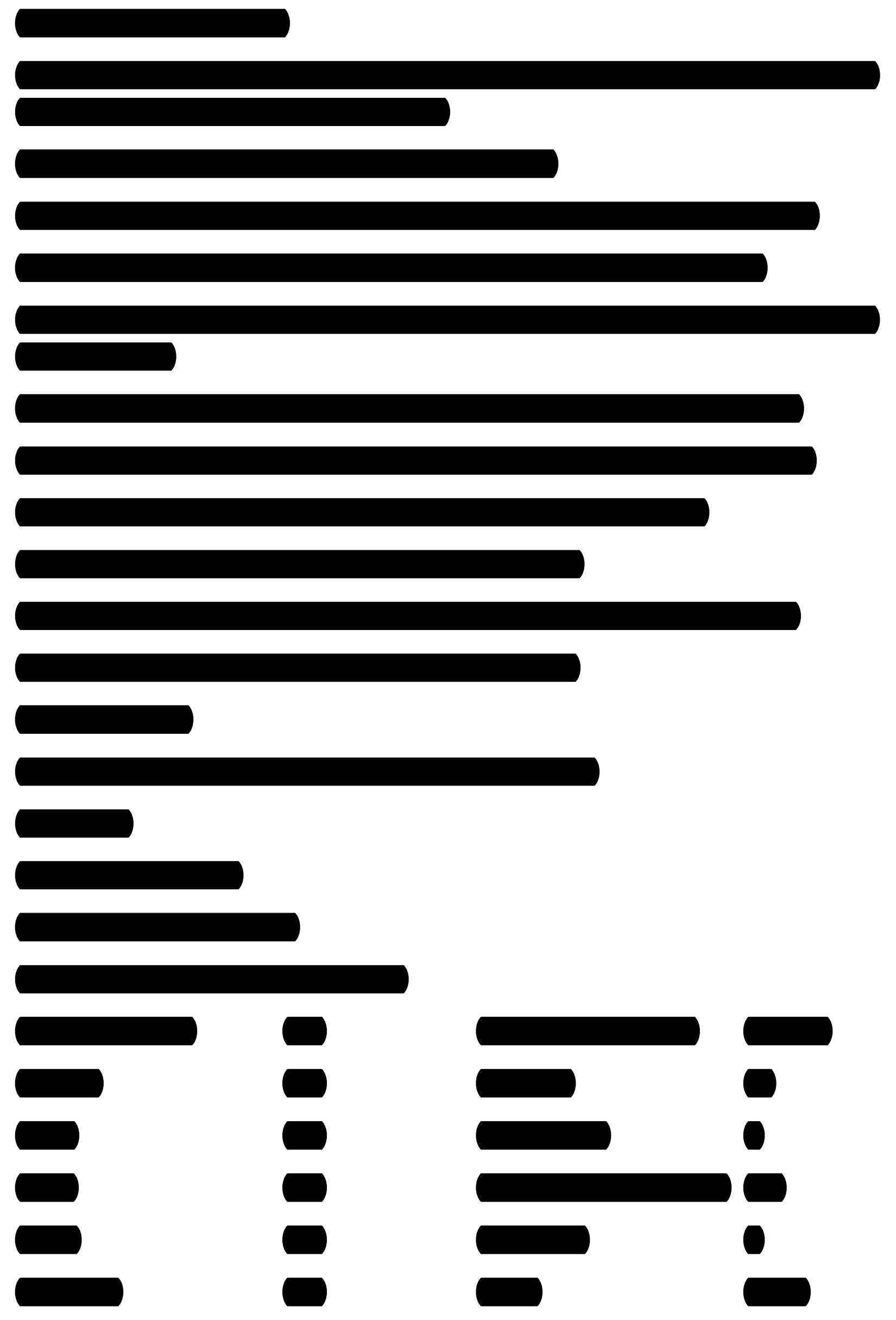




\section{Curriculum Vitae}

\section{Personal}

Name: $\quad$ Jens Tassilo Grün

Date of Birth: $\quad 31.01 .1991$

Place of Birth: $\quad$ Wetzlar, Germany

Nationality: German

\section{Education}

01/2017-Date of print

$11 / 2017-03 / 2021$

10/2014-09/2016

$04 / 2016-09 / 2016$

$10 / 2015-12 / 2015$

$10 / 2011-12 / 2014$

07/2014-09/2014
Doctorate $(\mathrm{PhD})$ in Chemistry with Prof. Harald Schwalbe Goethe University, Frankfurt am Main

Fachbereich 14 - Biochemie, Chemie, Pharmazie

Member and representative of the graduate research programme GRK 1986: “Complex Light Control”, (CLiC)

Master of Science (M.Sc.) in Chemistry, with distinction

Goethe University, Frankfurt am Main

Master Thesis with Prof. Harald Schwalbe: "NMR-Spectroscopic Investigations of G-Quadruplex-Ligand Complexes"

Study abroad and Research stay with Prof. Robert G. Griffin Massachusetts Institute of Technology, Cambridge (MA, USA)

Bachelor of Science (B.Sc.) in Chemistry

Goethe University, Frankfurt am Main

Bachelor Thesis with Prof. Harald Schwalbe: "Investigation of G-Quadruplex-Ligand Complexes”

Abitur (allgemeine Hochschulreife)

Johanneum Gymnasium, Herborn 


\section{Publications}

(9) G. Pinter, K.F. Hohmann, J.T. Grün, J. Wirmer-Bartoschek, C. Glaubitz, B. Fürtig, H. Schwalbe, Real-time NMR spectroscopy in the study of biomolecular kinetics and dynamics, Magnetic Resonance 2021, Robert Kaptein Festschrift, under revision, as preprint available: doi.org/10.5194/mr-2021-1

(8) J.T. Grün, A. Blümler, I. Burkhart, J. Wirmer-Bartoschek, A. Heckel, H. Schwalbe, Unraveling the Kinetics of Spare-Tire DNA G-Quadruplex Folding, Journal of the American Chemical Society 2021, accepted manuscript (published online)

(7) M. Novakovic, E. Kupče, T. Scherf, A. Oxenfarth, R. Schnieders, J.T. Grün, J. WirmerBartoschek, C. Richter, H. Schwalbe, L. Frydman, Magnetization transfer to enhance NOE cross-peaks among labile protons: Applications to imino-imino sequential walks in SARSCoV-2-derived RNAs, Angewandte Chemie (Int. Ed.) 2021, accepted article (doi: 10.1002/anie.202015948)

(6) R. Schnieders, S.A. Peter et al. (J.T. Grün as co-author in alphabetical order), ${ }^{1} \mathrm{H},{ }^{13} \mathrm{C}$ and ${ }^{15} \mathrm{~N}$ chemical shift assignment of the stem-loop 5a from the 5'-UTR of SARS-CoV-2, Biomolecular NMR assignments 2021, (doi: 10.1007/s12104-021-10007-w)

(5) R.W. Harkness, C. Hennecker, J.T. Grün, A. Blümler, A. Heckel, H. Schwalbe, A.K. Mittermaier, Parallel reaction pathways accelerate folding of a guanine quadruplex, Nucleic Acids Research 2021, 49 (3), 1247-1262

(4) A. Wacker, J. Weigand et al. (COVID19-NMR team, J.T. Grün as co-author in alphabetical order), Secondary structure determination of conserved SARS-CoV-2 RNA elements by NMR spectroscopy, Nucleic Acids Research 2020, 48 (22), 12415-12435* ( ${ }^{*}$ Breakthrough article)

(3) J.T. Grün, C. Hennecker, D.-P. Klötzner, R.W. Harkness, I. Bessi, A. Heckel, A.K. Mittermaier, H. Schwalbe, Conformational Dynamics of Strand Register Shifts in DNA G-Quadruplexes, Journal of the American Chemical Society 2020, 142 (1), 264-273*

*Spotlight in: D. Lockwood, Journal of the American Chemical Society 2020, 142 (6), 2697-2698

(2) M. Reese, C. George, C. Yang, S. Jawla, J.T. Grün, H. Schwalbe, C. Redfield, R. J. Temkin, R.G. Griffin, Modular, triple-resonance, transmission line DNP MAS probe for $500 \bigotimes \mathrm{MHz} / 330 \bigotimes \mathrm{GHz}$, Journal of Magnetic Resonance 2019, 307, 106573

(1) J. Wirmer-Bartoschek, L.E. Bendel, H.R.A. Jonker, J.T. Grün, F. Papi, C. Bazzicalupi, L. Messori, P. Gratteri, H. Schwalbe, Solution NMR Structure of a Ligand/Hybrid-2-GQuadruplex Complex Reveals Rearrangements that Affect Ligand Binding, Angewandte Chemie (Int.Ed.) 2017, 56, 7102-7106 


\section{Conference Contributions and invited Seminar talks}

(7) J.T. Grün, A. Blümler, A. Heckel, H. Schwalbe, Conformational Dynamics of unusual DNA structures: Insights into G-Quadruplex Polymorphism by Real-Time NMR-Spectroscopy Poster Presentation, 62 ${ }^{\text {nd }}$ ENC (Virtual Conference), March 2021

(6) J.T. Grün, Conformational Dynamics of unusual DNA structures: Insights into G-Quadruplex Polymorphism by Real-Time NMR-Spectroscopy

Seminar Talk (Zoom), Magnetic Resonance Seminar at Weizmann Institute of Science, October 2020

(5) J.T. Grün, A. Blümler, A. Heckel, H. Schwalbe, Conformational Dynamics of DNA GQuadruplexes - Insights by Real-Time-NMR

Oral Presentation, V. Doktorandenseminar Nucleinsäurechemie (DNG), Bad Herrenalb (Germany), October $\mathbf{2 0 2 0}$

(4) J.T. Grün, D.-P. Klötzner, R.W. Harkness, C. Hennecker, I. Bessi, A. Heckel, A.K. Mittermaier and H. Schwalbe, Mechanistic Insights to G-Quadruplex Conformational Dynamics by RealTime NMR-spectroscopy

Poster Presentation, $7^{\text {th }}$ Int. Meeting on Quadruplex Nucleic Acids Changchun (China), September 2019

(3) J.T. Grün, D.-P. Klötzner, R.W. Harkness, I. Bessi, A. Heckel, A.K. Mittermaier and H. Schwalbe, Investigation of DNA G-Quadruplex Structural Dynamics using Real-Time NMRspectroscopy

Poster Presentation, XXIIX. ICMRBS Dublin (Ireland), August 2018

(2) J.T. Grün, D.-P. Klötzner, R.W. Harkness, I. Bessi, A. Heckel, A.K. Mittermaier and H. Schwalbe, Investigation of DNA G-Quadruplex Structural Dynamics using Real-Time NMRspectroscopy

Poster Presentation, 20 $0^{\text {th }}$ JCF-Frühjahrssymposium Konstanz (Germany), March 2018

(1) J.T. Grün, D.-P. Klötzner, A. Heckel, I. Bessi and H. Schwalbe, Investigation of Promoter Gquadruplex Folding Dynamics using Real-Time NMR-spectroscopy

Poster Presentation, $6^{\text {th }}$ Int. Meeting on Quadruplex Nucleic Acids Prague (Czech Republic), June 2017 


\section{Honors and Awards}

2019

2018

Royal Society of Chemistry Poster Award, $7^{\text {th }}$ Int. Meeting on Quadruplex Nucleic Acids, Changchun (China)

2017 Suraj Manrao Poster Prize, XXIX. ICMRBS, Dublin (Ireland)

2015 Valedictorian, Fachbereich Biochemie, Chemie und Pharmazie Dr. Albrecht Magen Fellowship, Steuben-Schurz Gesellschaft, Frankfurt am Main Elisabeth-Meurer Fellowship, Elisabeth-Meurer Privatstiftung, Pulheim

\section{Academic Teachers (in alphabetical order)}

Prof. Dr. Norbert Auner, PD Dr. Markus Braun, PD Dr. George Bruls, Prof. Dr. Irene Burghardt, Prof. Dr. Björn Corzilius, Prof. Dr. Gunter Eckert, Prof. Dr. Ernst Egert, Prof. Dr. Joachim Engels, Prof. Dr. Michael Göbel, Prof. Clemens Glaubitz (D.Phil Oxon), Prof. Robert G. Griffin (PhD), Prof. Dr. Martin Grininger, Prof. Dr. Alexander Heckel, AOR Dr. Rainer Hegger, Prof. Dr. Mike Heilemann, Prof. Dr. Max C. Holthausen, PD Dr. Matthias Köck, Prof. Dr. Georg Manolikakes, Prof. Dr. Thomas Prisner, Prof. Dr. Matthias Rehahn, Prof. Dr. Martin U. Schmidt, Apl. Prof. Dr. Wolfgang Schubert, Prof. Dr. Harald Schwalbe, Prof. Dr. Andreas Terfort, Prof. Dr. Josef Wachtveitl, Prof. Dr. Matthias Wagner 